NISTIR 3978

\title{
AUTOMATIC WAVEFORM ANALYSIS AND MEASUREMENT SYSTEM USER MANUAL
}

S.M. Chesnut

N.G. Paulter 



\section{AUTOMATIC WAVEFORM ANALYSIS AND MEASUREMENT SYSTEM USER MANUAL}

S.M. Chesnut

N.G. Paulter

Electromagnetic Fields Division

Electronics and Electrical Engineering Laboratory

National Institute of Standards and Technology

Boulder, Colorado 80303

December 1991

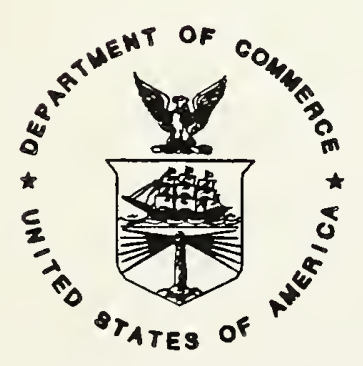

U.S. DEPARTMENT OF COMMERCE, Robert A. Mosbacher, Secretary NATIONAL INSTITUTE OF STANDARDS AND TECHNOLOGY, John W. Lyons, Director 

1.0 INTRODUCTION

1.1 General

1.2 Overview of The Manual

2.0 BACKGROUND

2.1 General

2.2 Sampling

2.3 Deconvolution

2.4 Time Calibration

2.5 Voltage Calibration

2.6 Pulse Parameter Calculation

3.0 HARDWARE SYSTEM DESCRIPTION

3.1 General

4.0 SOFTWARE SYSTEM DESCRIPTION

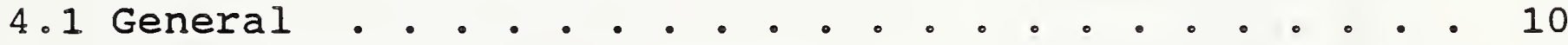

4.2 Data Acquisition - (ACQUIRE) . . . . . . . . . 11

4.2.1 Waveform menu .............. 14

4.2.2 Time-calibration menu . . . . . . . . . 18

4.2 .3 Voltage-calibration menu . . . . . . . . . 22

4.2 .4 operator information . . . . . . . . . . 24

4.3 Oscilloscope Calibration - (FIXACQ) . . . . . . . . 24

4.4 Deconvolution - (DECON NIST) . . . . . . . . . . 26

4.5 Pulse Parameter Calculātion - (PULS_PARAMS) . . . . 31

4.6 Support Programs . . . . . . . . . . . . . . . 35

4.6.1 Jitter waveform generator - (GAUSS) . . . . 35

4.6.2 Waveform math operations utility - (MATH_OPS) 35

4.6 .3 Graphics support - (GRAPH_DATA) . . . . . 39

5.0 SYSTEM CONSIDERATIONS

5.1 System Changes . . . . . . . . . . . . . . 41

5.2 Acquisition setup . . . . . . . . . . . . . . 41

5.3 oscilloscope and computer ........... 43 
APPENDIX A

A. 1 Specifications . . . . . . . . . . . . . . . A1

A.2 AWAMS Hardware . . . . . . . . . . . . . . . A2

A.3 Manufacturers' Users' Documentation .. . . . . A3

A. 4 Measurement Setup and Example Procedures A.4.1 General . . . . . . . . . . . . . . A5

A.4.2 Pulse generator, procedure 1 . . . . . . A5

A.4.3 Pulse generator, procedure 2 . . . . . . A11

A.4.4 Jitter measurement . . . . . . . . . A15

APPENDIX B (Software source code listings)

B. 1 ACQUIRE . . . . . . . . . . . . . . . . . . . B2

B.2 DECON NIST . . . . . . . . . . . . . B74

B.3 FIXAC̄ . . . . . . . . . . . . . . B109

B.4 PULS PARAMS . . . . . . . . . . . . . . . B137

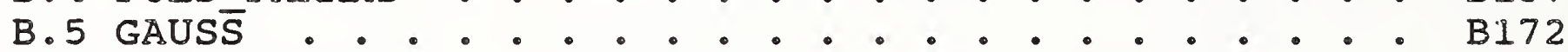

B.6 GD HISTOGRM ................... . . B196

B.7 MATH_OPS ................. B B200

B.8 text_out . . . . . . . . . . . . . . B231 
1. Diagram of AWAMS hardware system . . • . • . . . . . 9

2. Softkey menu for the data acquisition program ACQUIRE •. . . . . . . . . . . . . 12

3. Waveform menu of the acquisition program . . . . 15

4. TCAL menu of the acquisition program . . . . . . . 19

5. VCAL menu of the acquisition program . . . . . . . . 22

6. FIXACQ flow chart . . . . . . . . . . . . 25

7. DECON_NIST flow chart . . . . . . . . . . . 27

8. PULS_PARAMS flow chart . . . . . . . . . . . 32

9. GAUSS flow chart . . . . . . . ..... 36

10. MATH_OPS flow chart . . . . . . . . . . . 37

A1. Pulse generator measurement setup 1 . . . . . . . A6

A2. Pulse generator measurement setup 2 - 0 - 。 - . . A7 


\section{LIST OF TABLES}

1. Softkey options for the ACQUIRE program . . . . . . 13

2. Waveform screen menu selections . . . . . . . . . 17

3. TCAL screen menu selections . . . . . . . . . . 21

4. VCAL screen menu selections . . . . . . . . . . 23 
This work was supported by the calibration coordination Group, project number 276, for the Army Primary standards Laboratory at the Redstone Arsenal, Alabama.

We use trade names to specify the equipment used in this system. No endorsement by the National Institute of standards and Technology is implied. Similar products by other manufacturers may work as well or better. 



\subsection{General}

The Automatic Waveform Analysis and Measurement system (AWAMS) is a system used at the National Institute of standards and Technology (NIST) to obtain measurements of electrical pulses that have transients ranging from 10 nanoseconds (ns) to 20 picoseconds (ps) and to analyze these measurement data to obtain pulse parameters, such as transition duration (rise and fall time), pulse aberration (overshoot and undershoot), pulse amplitude (topline minus baseline), pulse duration (pulse width), etc. The AWAMS was constructed for the calibration coordination Group (CCG), project number 276 , by NIST.

\subsection{Overview of The Manual}

The remainder of the User's Manual provides the information necessary to operate the AWAMS. Section 2 includes a brief review of pertinent technical information, such as the theory of deconvolution. Sections 3 and 4 describe the hardware and software components of the AWAMS and their operation. In Section 5, we give warnings and considerations on the use of the AWAMS. section 6 contains references to related technical papers, section 7 a glossary of terms, and section 9 a specifications list, manufacturers users' manuals list, and example measurement procedures. Section 10 contains the software source code listings. 
2.1 General

The AWAMS uses an equivalent-time sampling oscilloscope to acquire discrete-time data that will represent a periodic continuous-time waveform. Before sampling the waveform, however, the sampling oscilloscope must be readied. Readiness is provided by triggering the oscilloscope before the waveform arrives at the oscilloscope's sampling gate. Only one sample point on the waveform is taken after each trigger event. The next point along the waveform is obtained by adding a small delay between trigger and waveform arrival. The entire waveform is then mapped by successively increasing this delay for each subsequent point. To get an accurate representation of the waveform, the time interval between trigger and waveform arrival must not vary randomly. In reality, however, this time interval does vary randomly; this random variation between the time of arrival of the waveform and its trigger is called jitter. The effect of jitter is to smooth the data, as would be done by a filter. This smoothing of the data is described mathematically by the convolution of the filter with the data.

The AWAMS provides a mechanism (deconvolution) for removing the effect of jitter on the acquired data so that a more accurate representation of the input waveform is obtained. In addition, the effect of the oscilloscope's sampling aperture is also removed, as are the effects of time and voltage errors. In section 2.2, we provide a review of sampling theory, and review the convolution and deconvolution processes in section 2.3. We describe the time and voltage calibration procedures in sections 2.4 and 2.5, and describe the pulse parameter calculations in Section 2.6 . 
The effect of equivalent-time sampling on a continuous function of time is to discretize the time over which the function is defined. For example, if a continuous function (corresponding to some real signal) is sampled once every second, then the discretized replica of the continuous function has information only at each $1 \mathrm{~s}$ interval. This can be written as

$$
g_{m}=g(m e), 0 \leq m \leq \infty,
$$

where $m$ is an integer, $\epsilon$ is the time interval between samplings, $g(m \epsilon)$ is $g(t)$ defined only at $t=m \epsilon, g(t)$ is the continuous function, and $g_{m}$ is the discretized replica of $g(m \epsilon)$. In eq (1), it is assumed $g(t)$ does not exist before $t=0$. Equation (1) may also be written, in a more useful form [1], as the product of a continuous function with a periodically spaced delta function $\delta(t)$ :

$$
g_{m}=g(t) \sum_{m=0}^{\infty} \delta(t-m \varepsilon)
$$

Recall that $\delta(t-a)=1$ when $t-a=0$ and is 0 otherwise. It is worthwhile to examine the frequency representation of eq (2); this is done using the Fourier transform. The Fourier transform of the summation in eq (2) becomes [2]:

$$
F T\left[\sum_{m=0}^{\infty} \delta(t-m \varepsilon)\right]=(2 \pi / \epsilon) \sum_{k=-\infty}^{\infty} \delta\left(t-k f_{s}\right) .
$$

where $\mathrm{k}$ is an integer, FT denotes a Fourier transform operation, and $\mathrm{f}_{s}=2 \pi / \epsilon$ is the frequency corresponding to the sampling interval. The Fourier transform of $g(t)$ is simply $(1 / 2 \pi) G(f)$. By an identity of Fourier transforms, the point-by-point multiplication of two functions in one domain becomes the convolution of the Fourier transforms of the two functions in the 
transform domain. Accordingly, the frequency representation of eq (2) is

$$
G_{k}=(I / \epsilon) G(f) * \sum_{k=-\infty}^{\infty} \delta\left(f-k f_{s}\right)
$$

where * denotes a convolution and $G_{k}$ is the discrete Fourier transform of $g_{m}$. Another property of Fourier transforms is that the Fourier transform of a real-valued waveform is Hermitian; that is, $G^{*}(-f)=G(f)$. Therefore, $\left|G^{*}(-f)\right|=|G(f)|$. (The superscript * denotes a complex conjugate, and the vertical bars indicate absolute values.) Let $f_{N}$ be the band-limiting frequency of $G(f)$; that is, $f_{N}$ is the highest frequency having information on $g(t)$. Because $G(f)$ is Hermitian, $|G(f)|$ is symmetric about $f=0$ and has information from $f_{-N}$ to $f_{N}$. Examination of eq (4) shows that $f_{S}$ must be greater than $2 f_{N}$ to avoid overlap between replicas of $G(f)$ that are centered at adjacent $\mathrm{kf}_{\mathrm{S}}$ values. This, then, is the sampling criterion: the sampling frequency must be at least twice the highest frequency that contains information on the input waveform. Accordingly, the waveform must not have faster transients than the sampling period.

\subsection{Deconvolution}

This section is taken from Ref. [3]. Data acquired from the measurement of a given signal are affected by the necessary intervention of the measuring device (such as measuring an electrical pulse with an oscilloscope). These data represent the signal as viewed by the measurement instrument and, therefore, can be described by the convolution of the instrument's impulse response with the signal. Consequently, it is necessary to remove the effects of the instrument on the data to get a more accurate representation of the signal; this is done by deconvolution. Discrete convolution is described by 


$$
f_{\tau}=\sum_{m=0}^{N-1} g_{m} h_{\tau-m}
$$

where, for illustration, $g_{m}$ is a measurable characteristic of an event under investigation, $h_{m}$ is the impulse response of the measurement system, $f_{\tau}$ is the acquired signal, $m$ is the time index, $\mathrm{N}$ is the number of points in the record, and $\tau$ is the delay. The frequency domain equivalent of eq (5) is

$$
F_{k}=G_{k} H_{k}, \quad 0 \leq k \leq N-1 \text {, }
$$

where $k$ is the frequency index and the functions $F_{k}, G_{k}$, and $H_{k}$ are the discrete Fourier transforms of the time functions given in eq (5). Typically, deconvolution is done with the discrete Fourier transformation (DFT) of the data and instrument response and, therefore, assumes periodicity of the data. Consequently, if the waveform is step-like (a step-like waveform is a waveform that has zero or nearly zero slope at either end of the record, and the nominal values at the ends of the record are not the same) the abrupt end of the record will cause oscillations in the record of the deconvolved data. This phenomenon is expected because the abrupt transition is artificial. Therefore, in order to perform deconvolution using step-like waveforms, the record's truncation discontinuities must be dealt with properly. The procedure used at NIST to minimize the record truncation discontinuity in step-like waveforms is the Nahman-Gans recordextension technique [4]. Other techniques that may minimize errors and decrease computation time are presently under investigation. In the frequency domain, the deconvolution becomes a division of the spectra,

$$
G_{k}=F_{k} / H_{k} \text {, }
$$

and $g_{m}$ is recovered by doing an inverse Fourier transform of $G_{k}$. 
Equation (5) represents a linear convolution process whereas eq (6) represents a cyclic convolution process. The two processes provide the same result as long as time-aliasing in the cyclic process is prevented [1]. For the waveforms used here, eq (5) and (6) will give equivalent results.

If the impulse response is not known exactly, only an approximate solution or best guess can be found to the deconvolution. The deconvolution is called "blind" when $G_{k}$ and $\mathrm{H}_{\mathrm{k}}$ of (6) are not known exactly. Furthermore, more than one possible solution may exist for the blind deconvolution, and the best solution must be selected. For example, when an electrical waveform is measured with an oscilloscope, the oscilloscope's impulse response is usually not known but is approximated. Consequently, either the data may be accepted as a true representation of the input signal or deconvolution attempted with an approximate impulse response and the dubious results accepted.

The ability to solve the blind deconvolution problem and obtain an accurate approximation to the signal is very important, especially when the instrument has a large effect on the signal. (Actually, blind deconvolution is not solved; only the best-guess solution is obtained.) The best-guess solution to the blind deconvolution problem is obtained by iterative techniques. The iterations continue until a change in a predetermined waveform attribute occurs. This attribute change is used as an indicator of the stability of the solution and is called the stopping criterion. The change in the waveform attribute is caused by varying a parameter used in the iterative deconvolution. The technique presently used by NIST to solve the blind deconvolution is a matched-filter technique using a variable parameter we call $\Gamma[5]$. The filter is a low-pass filter, and $\Gamma$ is related to the roll-off frequency. The filtering is applied to frequency domain deconvolution to suppress the noise that results by performing 
deconvolution for frequencies where there is no appreciable spectral content in $\mathrm{H}_{\mathrm{k}}$. The stopping criterion presently in use is the minimum in the power of the imaginary part of the reconstructed record. This power is given by

$$
P_{I}=\sum_{m=0}^{N-1}\left[I\left(g_{m}\right)\right]^{2} \text {. }
$$

other filtering techniques and stopping criteria that may provide better results and shorten processing time are being investigated.

\subsection{Time Calibration}

The time, or horizontal, axis calibration is performed by putting a single-frequency sinusoidal waveform into the oscilloscope. The zero crossings of the sine wave are used to calibrate the time axis. The zero crossings are used because they provide the most robust sine-wave parameter in terms of resistance to noise, amplitude fluctuations, and jitter. Once the locations of the zero crossings are determined, the time axis can be calibrated. For example, consider using a 10-GHz sine wave to calibrate a 1 -ns window that is defined over 1000 points. The zero crossings should be 50 points, or $0.05 \mathrm{~ns}$, apart. If they are not for any region between a pair of adjacent zerocrossings, the time values are corrected in that region. Once the time axis is corrected, the data are linearly interpolated so that their new values coincide with their new time. 
The voltage calibration routine is a steady-state technique. A known dc voltage is applied across the terminals (center and ground) of the sampling head's input port, and the voltage measured by the oscilloscope is recorded. After performing multiple measurments of this type, a relationship between the actual and measured voltages is obtained. This relationship can be imagined as a plot of the actual versus measured voltages. The slope of this plot is used to correct the voltage values of the acquired data. Consider an example where the measured voltage equals 0.9 times the actual voltage. The new data will be $\mathrm{V}_{\mathrm{c}}=\mathrm{V} / 0.9$, where $\mathrm{V}_{\mathrm{c}}$ is the corrected value and $\mathrm{V}$ is the acquired value.

\subsection{Pulse Parameter Calculation}

The purpose of measuring a pulse waveform is to calculate the values of the parameters that describe the waveform features. The parameters calculated by the pulse parameters program are: pulse amplitude, pulse transition duration, overshoot, and undershoot. We use a histogram-based algorithm for pulse parameter determination. The histogram represents a distribution function of the y-axis, usually voltage, values. The pulse parameters are defined in the ANSI/IEEE (American National Standards Institute/Institute of Electrical and Electronics Engineers) Std 194-1977 [6], and the algorithms we use for calculation of the pulse parameters are based on the IEEE Std $181-1977$ [7]. 


\subsection{General}

The AWAMS hardware system consists of an digitizing oscilloscope, a computer controller, and computer peripheral devices that are linked by an IEEE 488 bus interface. A block diagram of the hardware system is shown in Fig. 1. Descriptions of the hardware used in the AWAMS are found in the manufactuers' users' manuals. A list of these documents is found in Appendix A; refer to these manuals for specifications, operation procedures and safety information. References to these manuals will be indicated by [MUD $\{A\}]$, where $A$ is the document number. COMPUTER

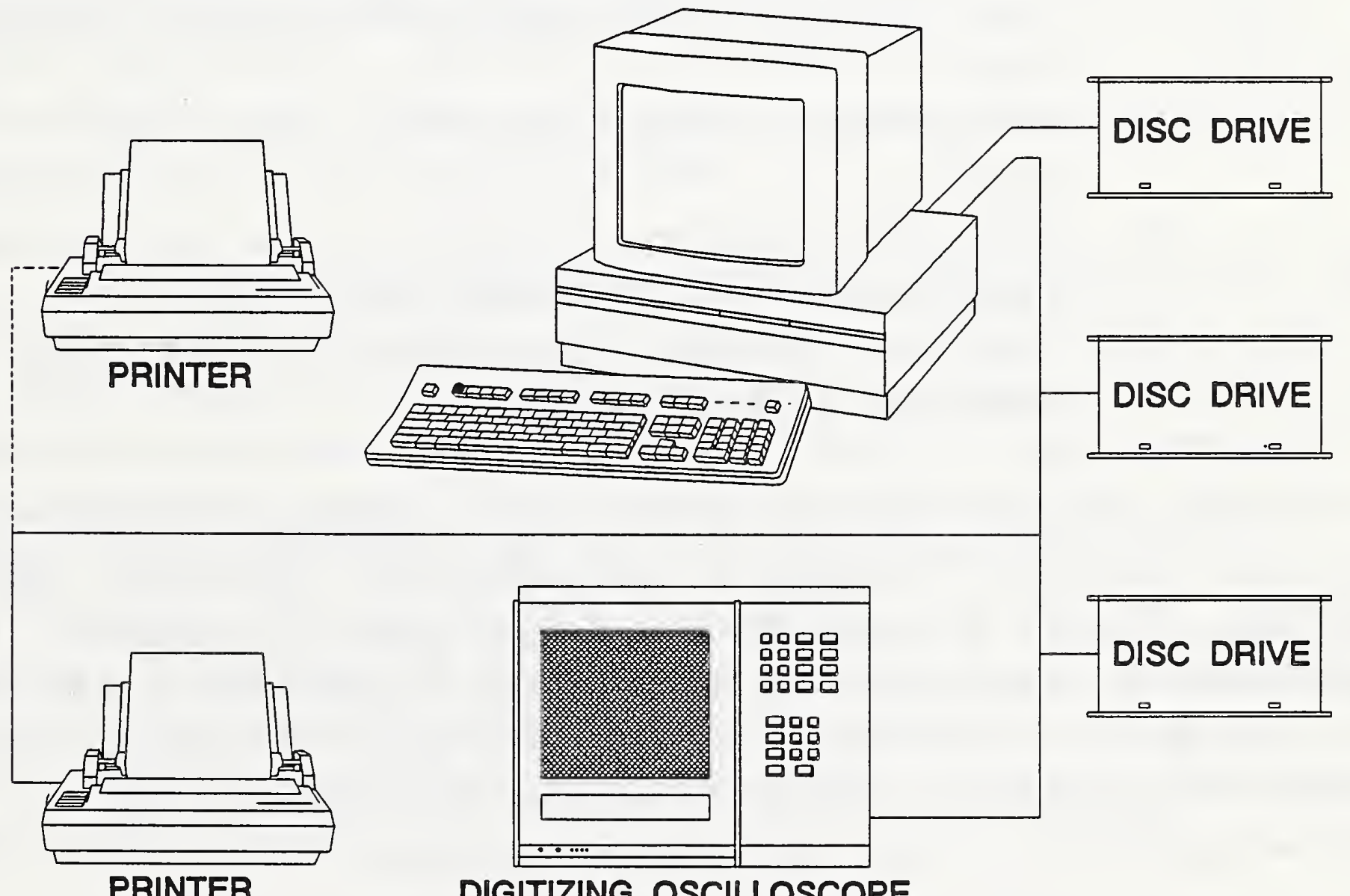

Figure 1. Diagram of AWAMS hardware system. 
4.0 SOFTWARE SYSTEM DESCRIPTION

4.1 General

The following programs are included in the AWAMS package:

1. ACQUIRE: a data acquisition routine that allows for acquisition of calibration data and waveform data.

2. FIXACQ: a utility to alter the waveform according to the calibration data.

3. DECON_NIST: a deconvolution routine.

4. GAUSS: a Gaussian waveform generator that creates a waveform to approximate the distribution function of the jitter of the measurement system. This function may be deconvolved from the waveform data.

5. PULS_PARAMS: a pulse parameters calculation program.

6. MATH_OPS: a vector utilities program that performs waveform mathematical operations. And,

7. text_out: a program that prints the ASCII file generated by PULS_PARAMS.

These programs must be loaded into the computer before they are run. The BASIC language is case sensitive; therefore, type the names exactly as shown above when you load the programs. A more detailed description of each program is provided in sections 4.2 through 4.6 , including menu descriptions or flow charts and written descriptions. Program details can be found in the following text or in the source code listings.

A version of GRAPH_DATA, the graphics support program developed at NIST is provided. No documentation for GRAPH_DATA is included in this manual. 
Error checking is included in the AWAMS software. If you hear a beep or series of beeps after running a program, pay attention to the message displayed on the screen. The beeps are alerting you to the presence of an error message. This message will let you know what went wrong.

\subsection{Data Acquisition -- (ACQUIRE)}

There are four measurements required for testing a pulse: the device under test (DUT) measurement, the voltage calibration measurement, the time calibration measurement, and a jitter measurement. The jitter measurement is an estimate of the time jitter in the DUT and measurement system [8] and is a single point measurement, described in the Appendix (A.4.4). With the exception of the jitter measurement, all measurements require the acquisition of digitized waveforms.

The acquisition program acquires the DUT waveform data, the voltage calibration data, and the time calibration data. This program is menu driven. Menu selections are made using simple onscreen graphics and the "softkeys." The main menu (softkeys) allows for the selection of any of the following: the waveform acquisition menu, the voltage calibration menu, the time calibration menu, and program information (see Fig. 2). Table 1 gives a brief description of the softkey options. No attempt was made to fully automate the front panel operation of the oscilloscope; therefore, many oscilloscope operations can only be performed manually.

The first time you enter any of the menus the acquisition program's user-definable parameters are preset to default values. When you change any parameter value, the system will retain the new values, as long as the program is in active memory (RAM), even if you move from menu to menu. 


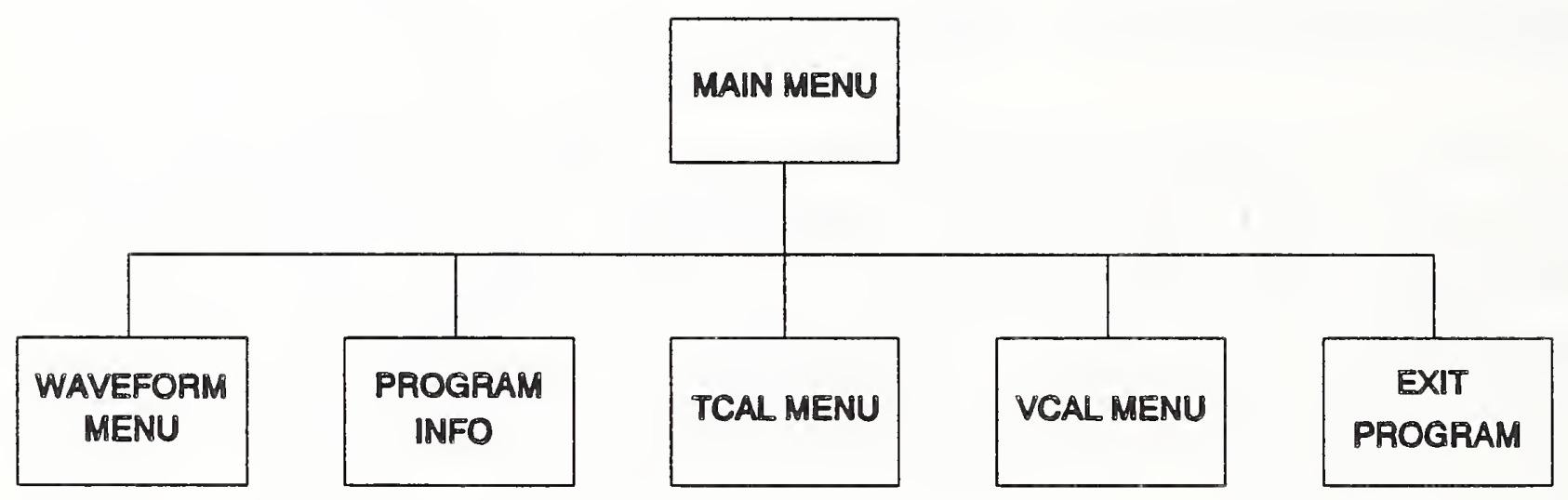

Figure 2. Softkey menu for the data acquisition program ACQUIRE.

There are oscilloscope settings that are not retained in memory. Any setup parameter not available in any menu will not be remembered when you change measurement menus. For example, if you use the oscilloscope's internal step generator as the input signal to a device, as in time-domain reflectometry (TDR), the step generator is turned off when you change to any other menu. The oscilloscope is reset every time you move from one menu to another to prevent the oscilloscope from being in an unknown state. However, to use the step generator as the input signal, use the manual setup feature of the acquisition program, which we describe later in this document, to turn on the step generator and view the waveform. Then use the "save setup" feature of the oscilloscope to retain this configuration [MUD \{1\}]. Acquire the 
Table 1. Softkey options for the ACQUIRE program.

\begin{tabular}{|c|c|}
\hline CHOICE & FUNCTION \\
\hline Main Menu & Returns the program from current menu. \\
\hline Exit Program & Quits the Acquire program. \\
\hline Acquire Data & $\begin{array}{l}\text { Causes the oscilloscope to take the data. } \\
\text { The oscilloscope takes control of the bus } \\
\text { during acquisition, but the "ABORT" key can } \\
\text { be used to interrupt the acquisition. }\end{array}$ \\
\hline Change Value & $\begin{array}{l}\text { Allows for changes of the on-screen } \\
\text { parameters. See the Tables on screen menu } \\
\text { choices. }\end{array}$ \\
\hline Manual setup & $\begin{array}{l}\text { Waveform: Allows for front panel operation } \\
\text { of the oscilloscope. May change any } \\
\text { parameter values and the new value will be } \\
\text { maintained when the program is continued. } \\
\text { TCAL/VCAL: Allows for front panel operation } \\
\text { of the oscilloscope. The values of menu } \\
\text { items, such as points, averages, etc.' } \\
\text { cannot be changed using this option. other } \\
\text { items, such as the trigger slope, that are } \\
\text { not included in the ACQUIRE program's menu } \\
\text { selection will change using this feature. } \\
\text { The manual setup key is most useful for } \\
\text { determining calibration of waveform details } \\
\text { like establishing the length of the delay } \\
\text { line in the TCAL trigger circuit that is } \\
\text { used to correctly place the signal in time. }\end{array}$ \\
\hline
\end{tabular}

data normally and then acquire the time and voltage calibration data. When you want to acquire another set of data using the step generator, return to the waveform menu, use the manual setup key, recall the saved setup and then continue with the measurement. By using the save and recall features, the oscilloscope's parameters will be restored to the same parameters used in the previous measurement.

The parameters, in every menu, have error checking. You change some of the parameters in their own menus. Other 
parameters are changed by toggling. Each of these provides error checking by limiting your alternatives to those values that the oscilloscope can provide. However, there are some parameters that are checked only for the value of the numeric input that make sense for the parameter. It is possible to input a value that the oscilloscope cannot accommodate; then the oscilloscope will default to the closest value it can provide. This is the only time there could be an inconsistency between the screen display and the acquired data. An error message will appear for a short time on the oscilloscope screen. Familiarity with the operation of the oscilloscope will help to prevent these errors.

In addition to error checking of the data, this program checks for a response from the oscilloscope, an external trigger, and the internal step generator. These checks prevent the attempted acquisition of waveform data from an oscilloscope that is off or from an oscilloscope that is on, but does not have a signal input into one of the test channels. The check for the existence of a waveform does not verify the sampling channel selection.

\subsubsection{Waveform menu}

First we describe the waveform menu (see Fig. 3). The parameters available in this menu are:

1. the channel for acquisition;

2. volts per division or vertical sensitivity;

3. offset or vertical position control;

4. attenuation, this facilitates the use of attenuator probes;

5. time per division or horizontal sensitivity;

6. delay or horizontal position control;

7. delay reference, either center or left side of the screen;

8. number of points to acquire in the waveform; 


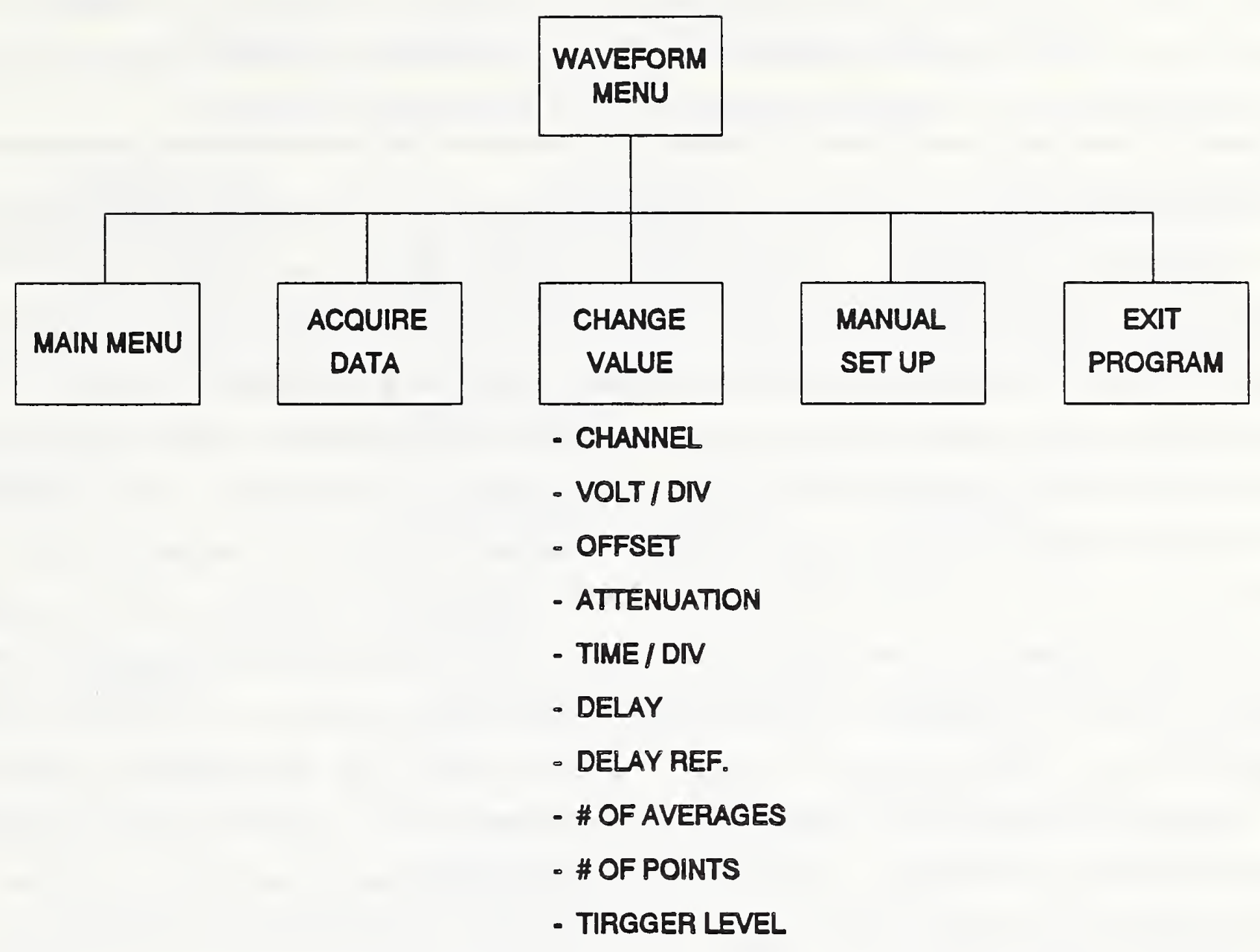

Figure 3. Waveform menu of the acquisition program.

9. number of averages used to re-create the waveform; and

10. trigger level.

The oscilloscope user manuals can provide more information on about these parameters, see [MUD $\{1\}$ ] and [MUD $\{2\}$ ]

You may select any one of the above parameters with the highlight box. The highlight box can be moved using the arrow keys, space bar, or knob. Table 2 gives a brief summary of the effect of changing each parameter and whether the values are changed from a menu, input by the operator, or toggled.

You can change the value of the selected parameter by using the softkey (at the bottom of the screen) marked "Change Value." Depending upon the parameter selected when you press the "Change 
Value" key, you will be prompted to input a value, to make a selection from another menu, or the "Change Value" key will cause the parameter to toggle between two possible values.

You will choose most of the parameter values, such as: volts per division, offset, and trigger level based on the DUT waveform. For example, the offset is chosen to center the waveform on the oscilloscope screen and the trigger level is chosen for the most nearly jitter-free waveform. You will select other parameter values for more subjective reasons. For example, increasing the number of averages taken during a measurement decreases the noise in the result. Another example, the number of points selected is based on the resolution you require. The trade-off for a larger number of averages and/or a larger number of points is acquisition time. Generally, we recommend selecting the highest available number of averages and points. The only instance when a large acquisition time is detrimental to the measurement is when the waveform drifts. However, if the waveform drift is significant, a high quality measurement is not possible under any circumstance. 
Table 2. Waveform screen menu selections.

\begin{tabular}{|c|c|c|}
\hline SELECTION & FUNCTION & HOW TO CHANGE \\
\hline Channel Number & $\begin{array}{l}\text { Selects a channel } \\
\text { for acquisition. }\end{array}$ & $\begin{array}{l}\text { Keyboard input. } \\
\text { Range: } 1 \text { to } 4 .\end{array}$ \\
\hline Volts per Division & $\begin{array}{l}\text { Changes oscilloscope } \\
\text { display vertical } \\
\text { resolution. }\end{array}$ & $\begin{array}{l}\text { Keyboard input } \\
\text { Range: } 1 \text { to } 80 \mathrm{mV}\end{array}$ \\
\hline offset & $\begin{array}{l}\text { Changes the vertical } \\
\text { placement of the } \\
\text { waveform trace--used } \\
\text { to correct a dc } \\
\text { offset. }\end{array}$ & $\begin{array}{l}\text { Keyboard input. } \\
\text { Range: } \\
\pm 500 \mathrm{mV}\end{array}$ \\
\hline Attenuation & $\begin{array}{l}\text { See description and } \\
\text { warnings, Secs. 5-3 } \\
\text { Ref. [6]. }\end{array}$ & $\begin{array}{l}\text { Keyboard input. } \\
\text { Range: } 1 \text { to } 1000\end{array}$ \\
\hline Time per Division & $\begin{array}{l}\text { Changes oscilloscope } \\
\text { display horizontal } \\
\text { resolution. }\end{array}$ & $\begin{array}{l}\text { Keyboard input. } \\
\text { Range: } 10 \text { ps to } 1 \mathrm{~s}\end{array}$ \\
\hline Delay & $\begin{array}{l}\text { Controls the time } \\
\text { position of the } \\
\text { waveform. }\end{array}$ & $\begin{array}{l}\text { Keyboard input. } \\
\text { options are time- } \\
\text { window dependent. }\end{array}$ \\
\hline Delay Ref & $\begin{array}{l}\text { Selects position } \\
\text { (center or left side } \\
\text { of screen). }\end{array}$ & Toggle. \\
\hline Number of Averages & $\begin{array}{l}\text { Changes number of } \\
\text { averages used to } \\
\text { acquire waveform. }\end{array}$ & $\begin{array}{l}\text { Menu. } 1 \text { to } 2048 \text {, } \\
\text { in steps of powers } \\
\text { of } 2 \text {. }\end{array}$ \\
\hline Trigger Level & $\begin{array}{l}\text { Changes oscilloscope } \\
\text { trigger level. }\end{array}$ & $\begin{array}{l}\text { Keyboard input. } \\
\text { Range: } \pm 1 \mathrm{~V}\end{array}$ \\
\hline
\end{tabular}

In addition to the screen menu, the waveform menu also includes the following softkey options:

1. exit to "Main Menu,"

2. "Exit Program,"

3. "Acquire Data,"

4. "Manual setup," and

5. "Change Value." 
We described the "Change Value" key above. The "Exit Program" and "Main Menu" keys are self-explanatory. The "Exit Program" key must be pressed to quit the program.

The "Manual set" option allows you to use the front panel input keys to adjust the oscilloscope. After modifying the measurement setup, press the "Continue" key to resume the program. The "Continue" key is either a softkey that appears on the screen, or a hard key on the keyboard. When the "Continue" key is pressed, all of the screen options are updated to the values chosen while in manual mode and the oscilloscope is returned to the remote mode. Be aware that changes made to parameters that are not included in the on-screen menu will need to be reset if you leave and then return to the waveform menu.

Selecting the "Acquire Data" key will reset the oscilloscope to the waveform parameters displayed on the screen, acquire the data, and send the data to the computer for storage. The data are stored in a format compatible with GRAPH_DATA. The oscilloscope will assume control of the IEEE-488 bus during the acquisition operation.

There is an "Abort" softkey available during acquisition. This option may be selected if you need computer control or if a parameter was incorrectly selected. When you select the "Abort" key, the measurement stops, the oscilloscope is reset, control of the bus is returned to the computer, and the program returns to the waveform menu.

\subsubsection{Time-calibration menu}

The second menu we describe is the time-calibration or "Tcal" menu (see Fig. 4) that is used to acquire the time-base calibration data. This menu is very similar to the waveformacquisition menu, but it contains only those parameters that are 


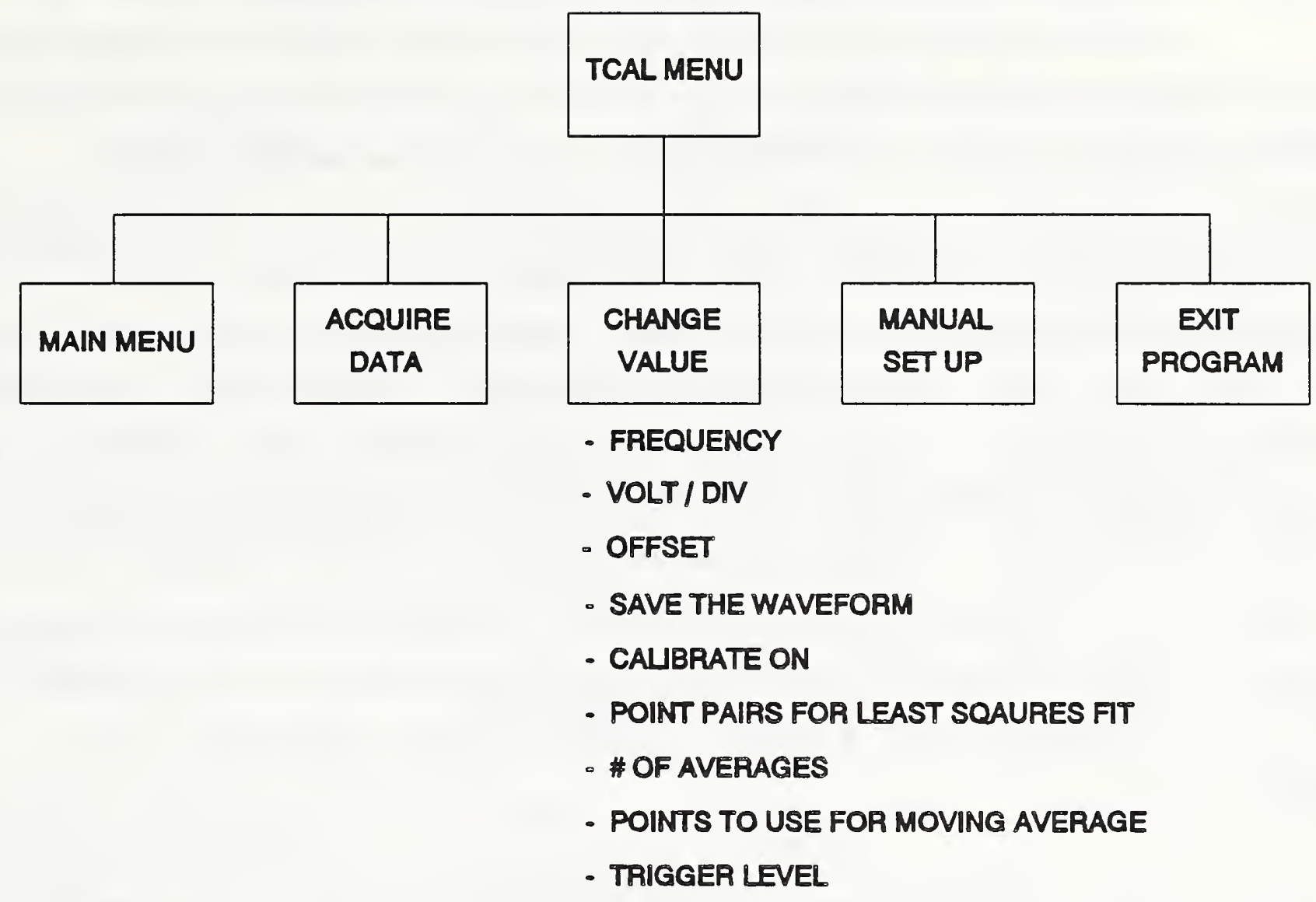

Figure 4. TCAL menu of the acquisition program.

pertinent to time-base calibration and allows changes to those parameters that will not compromise the consistency of the data set. The time calibration screen choices are:

1. calibration frequency (the frequency of your time standard);

2. number of averages for acquisition;

3. volts per division:

4. offset;

5. the number of points for the sliding average noise filter;

6. the number of points for the linear least squares fit (for finding the zero crossings of the calibration waveform);

7. the slope (positive or negative) to calibrate on; 
8. whether or not to save the waveform from which the time-calibration data are derived (this is generally a sine-wave); and

9. the trigger level for the time standard.

As with the waveform menu, you can select the above parameters by using the arrow keys, space bar or knob and the highlight box. When you press the "Change Value" key, you will be prompted to input a value, to make a selection from another menu, or the "Change Value" key will cause the parameter to toggle between two choices. Again, the behavior of the "Change Value" key depends on the selected parameter. Table 3 gives a brief summary of the effect of changing each parameter and whether the values are changed from a menu, input by the operator, or toggled.

The time calibration menu also includes the following softkey choices:

1. exit to "Main Menu,"

2. "Exit Program,"

3. "Acquire Data,"

4. "Manual set," and

5. "Change Value."

With the exception of the "Manual set" key, these softkeys work as described in the waveform menu section. The "Manual set" key in the time calibration menu is available to facilitate the setup of the time calibration waveform in the epoch. With this feature, you can reposition the calibration waveform with external delay line(s), usually inserted in the trigger circuit, and quickly see the effect of the added delay line. If you change the parameter values while in the manual mode, none of the changed values are read into the acquisition program. All changed settings are ignored when you return to the program from "Manual set." This feature provides a fast and convenient way for you to determine the optimal physical setup and built-in 
Table 3. TCAL screen menu selections.

\begin{tabular}{|c|c|c|}
\hline SELECTION & FUNCTION & HOW TO CHANGE \\
\hline $\begin{array}{l}\text { Calibration } \\
\text { Frequency }\end{array}$ & $\begin{array}{l}\text { Establishes the } \\
\text { frequency for time } \\
\text { calibration. }\end{array}$ & $\begin{array}{l}\text { Keyboard input. } \\
\text { Depends on time } \\
\text { calibration } \\
\text { standard used. }\end{array}$ \\
\hline Number of Averages & $\begin{array}{l}\text { Changes number of } \\
\text { averages used to } \\
\text { acquire waveform. }\end{array}$ & $\begin{array}{l}\text { Menu. } 1 \text { to } 2048 \\
\text { in steps of } \\
\text { powers of } 2 \text {. }\end{array}$ \\
\hline Volts per Division & $\begin{array}{l}\text { Changes oscilloscope } \\
\text { display vertical } \\
\text { resolution. }\end{array}$ & $\begin{array}{l}\text { Keyboard input. } \\
\text { Range: } 1 \text { to } 80 \\
\mathrm{mV} .\end{array}$ \\
\hline Offset & $\begin{array}{l}\text { Changes the vertical } \\
\text { placement of the } \\
\text { waveform trace. }\end{array}$ & $\begin{array}{l}\text { Keyboard input. } \\
\text { Range: } \pm 500 \mathrm{mV}\end{array}$ \\
\hline $\begin{array}{l}\text { Number of Points for } \\
\text { sliding Average } \\
\text { Filter }\end{array}$ & $\begin{array}{l}\text { Used to filter noise } \\
\text { and find voltage } \\
\text { crossings. }\end{array}$ & Keyboard input. \\
\hline $\begin{array}{l}\text { Number of Points for } \\
\text { Least squares Fit }\end{array}$ & $\begin{array}{l}\text { Used to find the } \\
\text { time of the zero } \\
\text { crossings. }\end{array}$ & Keyboard input. \\
\hline slope & $\begin{array}{l}\text { Used to find the } \\
\text { time of the zero } \\
\text { crossings. }\end{array}$ & Toggle: +/- \\
\hline $\begin{array}{l}\text { Save the Time } \\
\text { Calibration Waveform }\end{array}$ & $\begin{array}{l}\text { Save time- } \\
\text { calibration waveform } \\
\text { data. }\end{array}$ & Toggle: yes/no \\
\hline Trigger Level & $\begin{array}{l}\text { Changes oscilloscope } \\
\text { trigger level. }\end{array}$ & $\begin{array}{l}\text { Keyboard input. } \\
\text { Range: } \pm 1 \mathrm{~V} .\end{array}$ \\
\hline
\end{tabular}

protection against acquiring inconsistent data. You must acquire the time calibration data using the same time/division and delay settings as the DUT data. If not, the epoch for the DUT will not be the same as the calibrated epoch and the data set will be useless. For more details on establishing the time calibration setup, see sections $A .4 .2$ and A.4.3 of this manual. 
The last menu available in the acquisition program is the "Vcal" or voltage-calibration menu (see Fig. 5). This is the simplest menu in the program with only four screen options and five softkey options.

The four screen options are:

1. number of voltage intervals to measure;

2. the number of averages wanted for the acquisition;

3. the starting calibration voltage value; and

4. the step size of the calibration voltage increments.

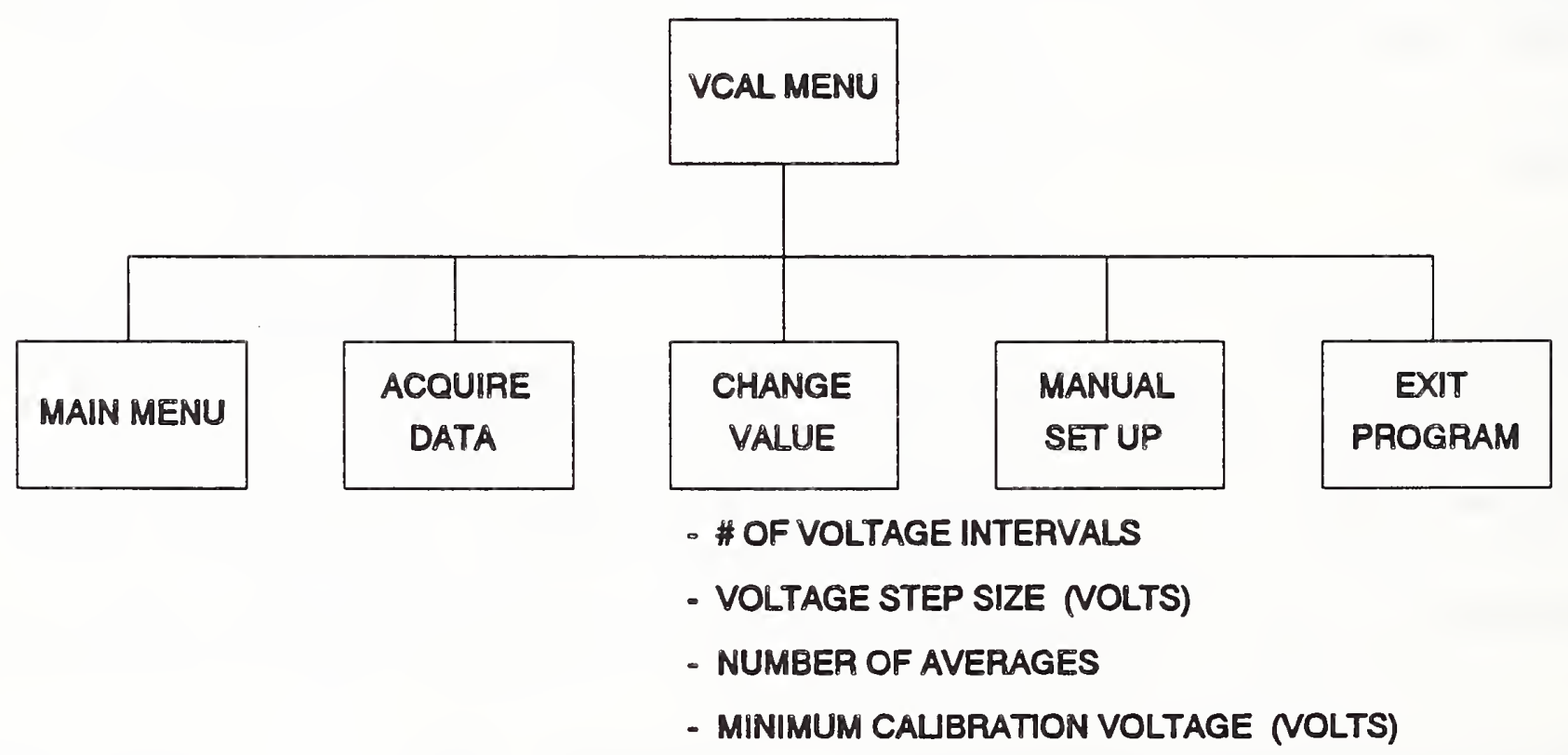

Figure 5. VCAL menu of the acquisition program. 
Table 4. VCAL screen menu selections.

\begin{tabular}{||l|l|l||}
\hline \multicolumn{1}{|c|}{ SELECTION } & \multicolumn{1}{|c|}{ FUNCTION } & \multicolumn{1}{|c|}{$\begin{array}{c}\text { HOW TO } \\
\text { CHANGE }\end{array}$} \\
\hline $\begin{array}{l}\text { Number of Voltage } \\
\text { Levels }\end{array}$ & $\begin{array}{l}\text { To set the voltage range } \\
\text { for calibration, used with } \\
\text { Step size. }\end{array}$ & $\begin{array}{l}\text { Keyboard } \\
\text { input. } \\
\text { Range: >0 }\end{array}$ \\
\hline Averages & See waveform menu. & Menu. \\
\hline $\begin{array}{l}\text { Starting } \\
\text { Calibration Voltage }\end{array}$ & $\begin{array}{l}\text { To set starting value that } \\
\text { is used to calibrate data. }\end{array}$ & $\begin{array}{l}\text { Keyboard. } \\
\text { Values } \\
\text { depend on } \\
\text { voltage } \\
\text { standard. }\end{array}$ \\
\hline Step size & $\begin{array}{l}\text { To set the voltage } \\
\text { increment for calibration. }\end{array}$ & $\begin{array}{l}\text { Keyboard. } \\
\text { Values } \\
\text { depend on } \\
\text { voltage } \\
\text { standard. }\end{array}$ \\
\hline
\end{tabular}

As with the waveform and time-calibration menus, you may select these parameters by using the highlight box. Table 4 gives a brief summary of the effect of changing each parameter and whether the values are changed from a menu, input by the operator, or toggled.

The five softkey choices are:

1. exit to "Main Menu,"

2. "Exit Program,"

3. "Acquire Data,"

4. "Manual Set," and

5. "Change Value."

These keys work as described in the time calibration acquisition section. Again for data consistency, the Manual key does not result in permanent changes. The "Manual set" key in this menu provides a convenient method for determining the 
voltage calibration interval, step size, and minimum voltage. See sections A.4.2 and A.4.3 of this manual for more details.

\section{2 .4 Operator information}

We provide this feature of the acquisition program to give new system users some idea of the programs function and to highlight a few of the more important considerations of data acquisition using the oscilloscope. This is a limited "Help" function. You may alter these messages to fit your needs by altering the source code. We do not intend this attribute as a replacement for reading this manual or the manuals for the system hardware. Any information contained in the original message when you select the operator information softkey is also in this manual.

4.3 Oscilloscope Calibration -- (FIXACQ)

FIXACQ is the program for applying the voltage and time calibration data to the DUT waveform. You may elect to calibrate the voltage scale, the time scale, or both (see Fig. 6). If you decide to apply both voltage and time correction to the DUT data, the voltage calibration will always be done before the time calibration.

Once the program starts, you will be prompted to input the appropriate file names. Error checking is provided to insure that you do not attempt to use an empty file.

A confusing situation occurs when the calibration epoch is less than the measured data epoch. This creates the situation in which the measured data extend beyond the calibration data and the question arises as to what to do with the "extra" measured data. Should this occur, you will have seven options. These 


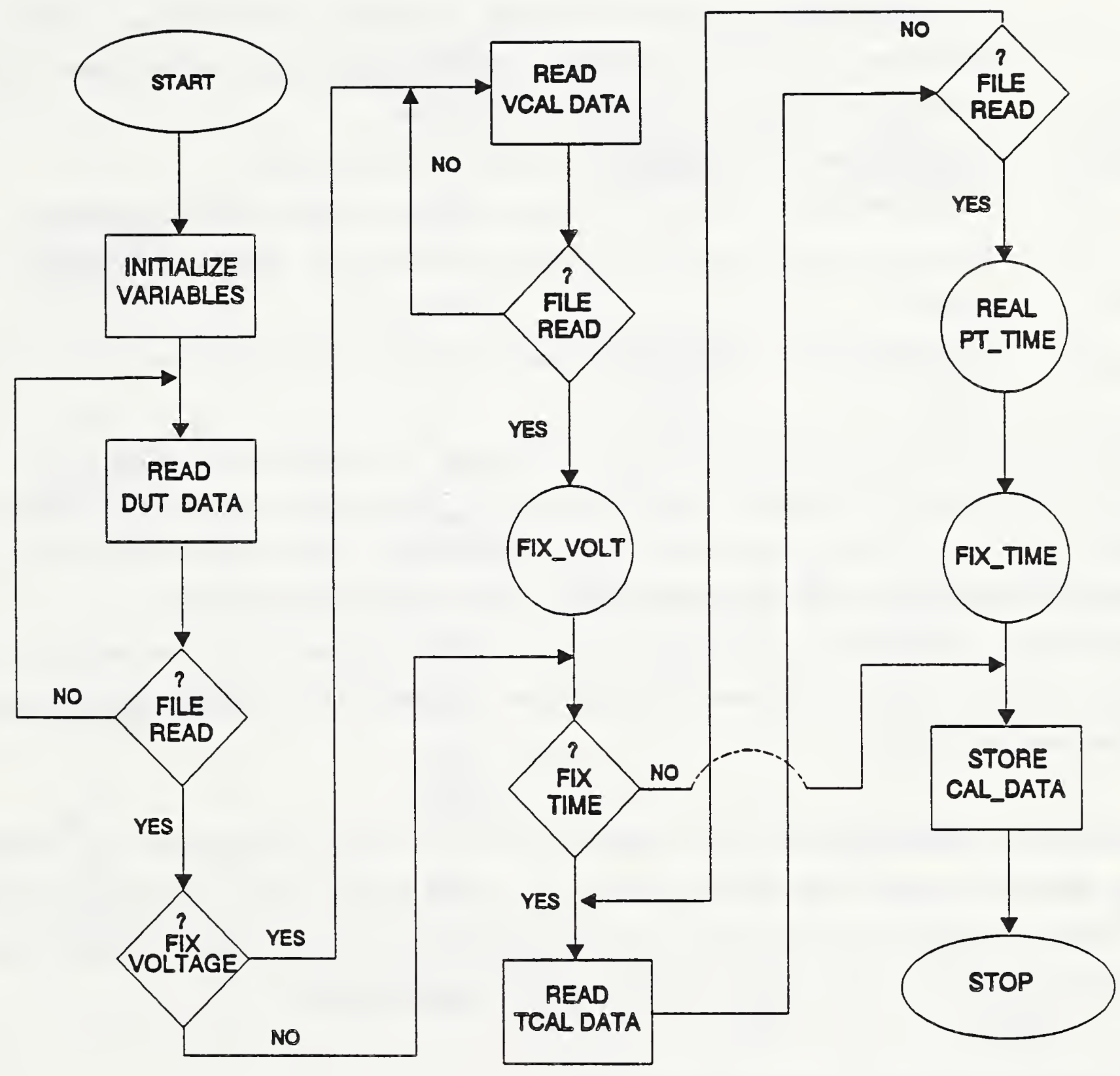

Figure 6. FIXACQ flow chart. 
options are listed below.

0. Abort the calibration; no new data will be saved.

1. Abort only the time base calibration. This will save the voltage calibrated data.

2. Calibrate and output fewer data points. This is not recommended since the fast Fourier transform (FFT) routine used in deconvolution requires waveforms that are $2^{\mathrm{n}}$ points in length.

3. Extrapolate using the last data point.

4. Extrapolate using a value input from the keyboard.

5. Extrapolate using the mean value of the last 5\% of the data.

6. Extrapolate using the mean slope of the last $5 \%$ of the data.

In this program, extrapolation is done by replacing the uncorrected points with the value for the given option. You will choose option 2 when you want to calibrate the DUT waveform and have no intention of deconvolving the calibrated data. Generally, we select option 6 as a "best guess" for what the data would actually be, unless there are compelling reasons for one of the other choices.

After calibrating the data, you will be prompted to input a file name for the corrected data -- the data will then be saved and the program terminated. The data are saved in binary data format and are also consistent with GRAPH_DATA.

4.4 Deconvolution -- (DECON_NIST)

You will use this deconvolution program for both the deconvolution of the jitter impulse response and the system impulse response. We provide the system-impulse-response data as part of the AWAMS. These data are complex (incompatible with GRAPH_DATA); therefore, you will need to respond accordingly when the deconvolution program asks for the data type. The flow chart 
(Fig. 7) shows the outline of the steps in this routine. Further

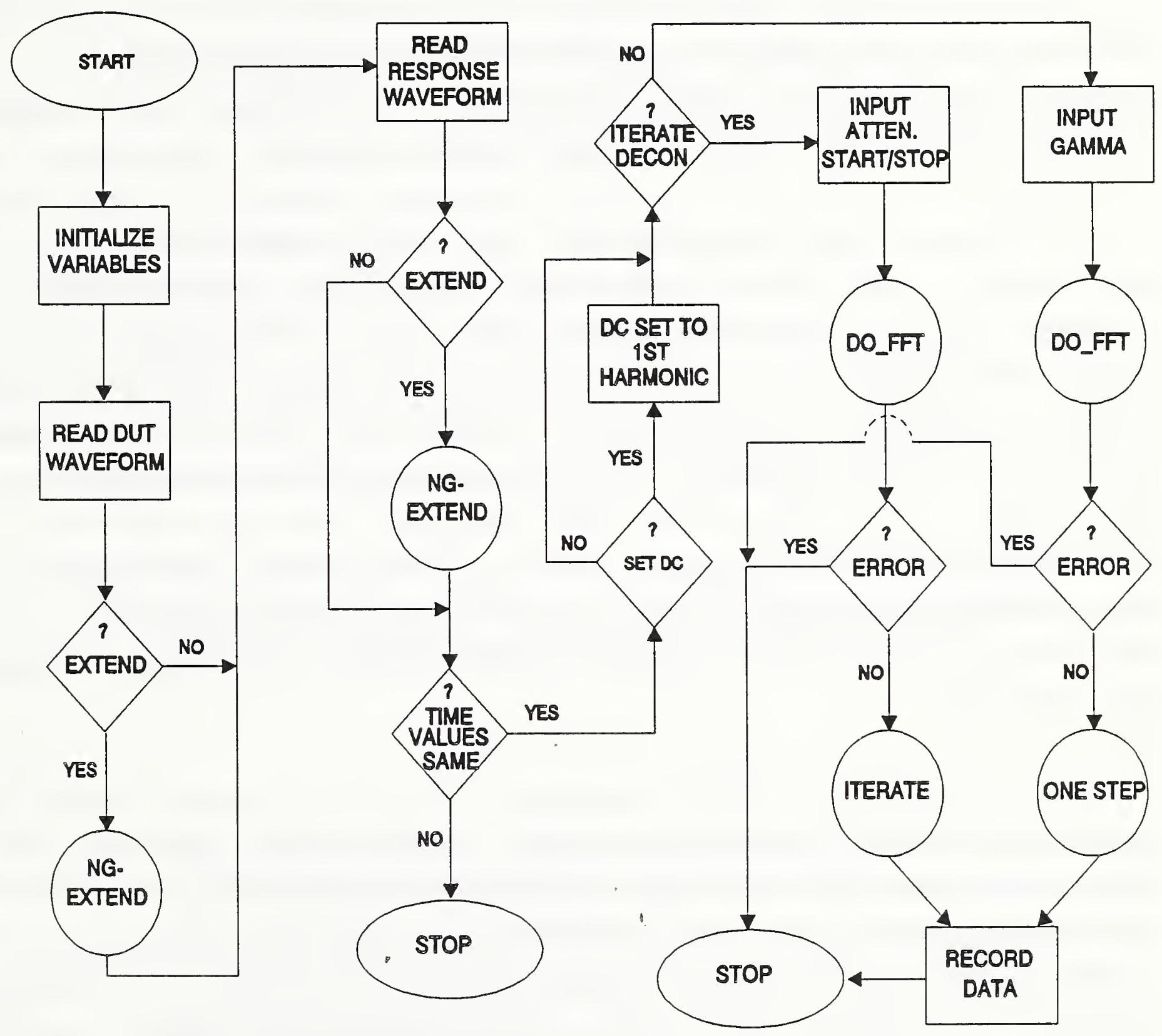

Figure 7. DECON_NIST flow chart. 
details about the algorithms for waveform extension, iterative or single-pass deconvolution, and FFT preparation follow. See section 2 of this manual and Refs. [1] through [5] for more detail on the theory of deconvolution.

Theoretically, deconvolution is a straightforward operation. However, in the presence of noise and other uncertainties, deconvolution becomes complicated [5]. Successful use of this program for deconvolution requires some insight.

First, you will be asked if you want to extend the waveform(s). If the DUT waveform is step-like, Nahman-Gans extension [4] (or another method for coping with record discontinuities (3]) is necessary for the deconvolution to work. Also, the number of points and the epoch for the DUT and response waveforms must be identical for the deconvolution to work. Therefore, if you extend the DUT waveform, you will have to extend the response waveform also. If you know a waveform has been stored in extended form, as with the system response waveform data provided as part of the AWAMS, no further extension is required.

If you extend the DUT waveform, you will also be asked what type of waveform is being extended, step-like or impulse-like. This is because the extension method is different for each of these waveform types. The extension of step-like waveform is explained in the preceding paragraph and in section 2.3 of this manual. An impulse-like waveform is extended by adding zeros to the waveform up to the desired number of points of the extension. Therefore, the program prompts you for the appropriate input as needed, but you will need to know the right answer(s). If you request a waveform extension when one is not necessary, the program may end because of an inconsistent number of data points, or there may be no adverse effect if both the DUT and the response waveforms are unnecessarily extended. 
The deconvolution program checks for consistency in the epoch and number of points acquired. The program will not perform the deconvolution and will end normally if these conditions are not met and you will have to re-run the program with a consistent data set.

The second aspect of this deconvolution program that requires explanation and decision is the choice between iterative and single-pass deconvolution. Select the single pass option only when you already know the optimal value of $\Gamma$. ( $\Gamma$ is the variable parameter that we use for selecting the optimal solution.) When the optimal value of $\Gamma$ is not known, select the iterative option. The iterative option causes the program to search for the optimal solution by varying the value of $\Gamma$ until our stopping criterion is met. As mentioned in section 2, our stopping criterion is a minimum in $P_{i}$, see eq (8).

When you select the iterative option, you will be asked to input the starting and stopping values for the attenuation. The optimizing parameter, $\Gamma$, is defined by

$$
\Gamma=10^{A},
$$

where $A$ is the attenuation. Do not select too large a range between the starting and stopping attenuation values. Although you may be more assured of finding the correct stopping point, iterative deconvolution takes a long time, and a larger range means a longer calculation time. It is all too easy to select a range for the attenuation that will not allow the program to find a stopping point. This happens when the range selected is too far from the correct value. Should this happen, reset the computer and run the program using a different range for the attenuation values. We are investigating other methods for finding the optimal stopping point; these may result in a more robust computation, but until then, you must develop a feeling 
for what will and will not work. Typically, if the two waveforms for deconvolution are similar, the optimal value of $\Gamma$ will be small $\left(10^{-3}\right.$ or smaller). On the other hand, if the waveforms are dissimilar, as is most often true, the optimal value of $\Gamma$ can be quite large ( $10^{23}$ or larger). Select the starting and stopping attenuation values based on the waveforms that are being deconvolved. For instance, when deconvolving the oscilloscope system response waveform from a measured waveform, we most often choose a starting attenuation of 15 and a stopping attenuation of 20. This range is based on experience with this deconvolution algorithm.

The third aspect of DECON_NIST we describe are the routines required for performing the Fourier transforms. Unlike the extension and iteration described above, the FFT routines are transparent to the user. The first required routine is called Do_fft. Do_fft sets up the call to another routine, Fft_fix; interprets any errors detected by Fft fix; and sets an error flag if an error has been detected. When this flag is set, the program terminates. The Fft_fix routine checks the data for situations that will generate an error in the FFT; if no errors are detected, it splits the data into its real and imaginary parts; and calls the FFT subroutine. When an error occurs, the error flag is assigned a number based on the cause of the error. This value is decoded in the Do_fft routine resulting in an onscreen error message and program termination.

Finally, we describe the options for data storage in this program. You will be given the choice to store an intermediate step and you will have the option to store several final results. All data are stored in binary data format and the stored files are compatible with GRAPH DATA. The first result you may choose to keep is the $\Gamma$-versus $-P_{i}$ curve used to determine the optimal value of $\Gamma$. This is an intermediate result that may be useful for two reasons. First, if you chose to re-run this program on 
the same data set, you can use the single-pass option since you will know the optimal $\Gamma$. And second, you may want to save this information for comparison with other similar data sets or as part of your records for each device you test. After the deconvolution process is done, you will have the option of saving the spectrum magnitude, the real time-domain result, and/or the imaginary time domain result. Usually, the only result you will want or need is the real time-domain result. You may save the spectrum magnitude for any waveform type, but it is most useful for determining the frequency content of pulses used for electromagnetic interference (EMI) testing, usually an impulselike signal. The imaginary result is made available in case you want to check the imaginary part to assure yourself that it is small; ideally the imaginary part of a real-valued waveform is 0 . If you elected to extend the DUT waveform, you will also be given the option of saving the resultant waveforms in the extended or half-length forms. If you are saving a system impulse-response waveform that will later be used for another deconvolution, save the extended versions. If you are not going to do any subsequent deconvolution(s), save the unextended form because the extended version is not useful. This program will end automatically when the data have been stored.

\subsection{Pulse Parameter Calculation -- (PULS PARAMS)}

Load and run PULS_PARAMS (Fig. 8) to calculate various pulse parameters. You may use this program on step-like, square-like, or impulse-like waveforms. To make the program more general, the units, such as volts and seconds, or amperes and milliseconds, are user inputs; this allows the program to operate on different kinds of data. This program calculates

1. peak to peak and 0 percent to 100 percent data values;

2. pulse amplitudes;

3. 10 percent to 90 percent and 20 percent to 80 percent pulse transition durations; 


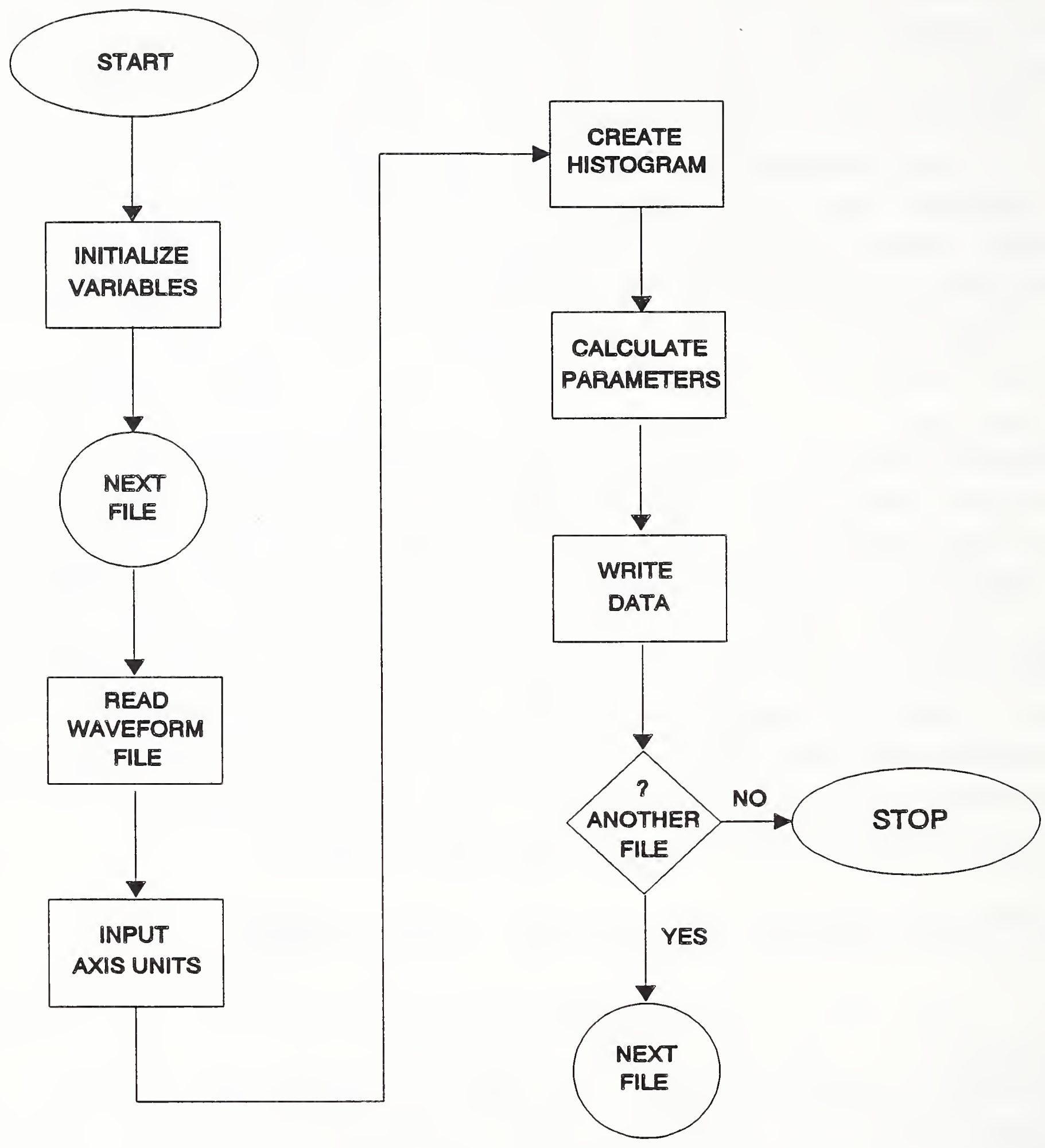

Figure 8. PULS PARAMS flow chart. 
4. percent overshoot; percent undershoot;

5. the second transition durations and pulse duration for impulse or square waveforms;

6. the number of bins used to create the histogram;

7. the 0 percent and 100 percent data values; and

8. the number of data occurrences at 0 percent and 100 percent.

It also displays the first, last, maximum, and minimum data values.

The first step in calculation of these parameters is finding an appropriate histogram. There should be roughly 10 points in either the 100 percent or 0 percent bin for an impulse-like waveform and 10 points for both the 0 percent and 100 percent bins for a step-like signal. The program will decrease the number of histogram bins until there are enough data occurrences in the 0 percent bin. The 0 percent bin is the only one checked by this program. Once the automatic bin calculation is done, you will be asked if the histogram is acceptable. Reasons for rejecting the histogram include an insufficient number of points at 100 percent or an inadequate $y$-axis resolution. If for any reason the histogram is unacceptable, you can override the automatic bin calculation and enter in the number of bins you want.

The second step is to define the 0 percent and 100 percent values. If the pulse waveform is negative-going, the 100 percent value becomes the 0 percent value and the 0 percent value becomes the 100 percent value. Be aware of this when selecting the definitions. Your options for 0 percent are:

1. value of the first point in the waveform;

2. value of the last point in the waveform;

3. the minimum value in the waveform;

4. the 0 percent value found by the histogram; or

5. you may input a value from the keyboard. 
Your options for 100 percent are:

1. value of the first point in the waveform;

2. value of the last point in the waveform;

3. the maximum value in the waveform;

4. the 100 percent value found by the histogram; or

5. you may input a value from the keyboard.

If the waveform is step-like or square, you will want to pick the values found by the histogram (option 4) for both 0 and 100 percent. If the waveform is impulse-like, you will want to pick the value found by the histogram (option 4) for 0 percent and the maximum waveform value (option 3) for 100 percent.

After you input the required level definitions, the program automatically continues with the calculation of the pulse parameters. When these calculations are finished, you will be asked to input a file name for data storage. These data are stored in ASCII format.

You will then be asked if you would like a hard copy printout of the results. If you choose not to print the result at run time, you can obtain a hard copy later by running the "text_out" program. This program simply reads in an ASCII file and prints it. Just load and run the program and answer the questions given. 
4.6 support Programs

4.6.1 Jitter waveform generator -- (GAUSS)

This program is used to create a waveform that approximates the jitter distribution function of the measurement system. This function can be deconvolved from the DUT waveform data. The first step in using this program is to measure sigma $(\sigma)$ of the jitter of the DUT. Sigma is the sum of the squared deviations of the measurements from their mean. This measurement is explained in section A.4.4 of this manual.

After measuring the value of $\sigma$ of the jitter, load and run GAUSS (see Fig. 9). You will be prompted to input values for the number of points in the waveform, the epoch of the waveform, and the value of $\sigma$ (Section A.4.4) as needed. The number of points and the epoch of the jitter distribution function must be equal to the number of points and the epoch of the DUT waveform for deconvolution. To make the jitter distribution function waveform more like an impulse response, we create a Gaussian curve with unit area. If you choose other than unit area, you will be prompted to input an amplitude value. When the waveform has been calculated, you will be asked for a file name for data storage. The result is stored, and the program ended. Again, the data are stored in binary data format and the file written is compatible with GRAPH_DATA.

4.6.2 Waveform math operations utility (MATH_OPS)

The MATH_OPS program allows you to perform some basic operations on the waveforms. These operations are: integration, differentiation, time shifting, and constant arithmetic operations of addition, subtraction, multiplication, and division (see Fig. 10). This program is similar in style to the 


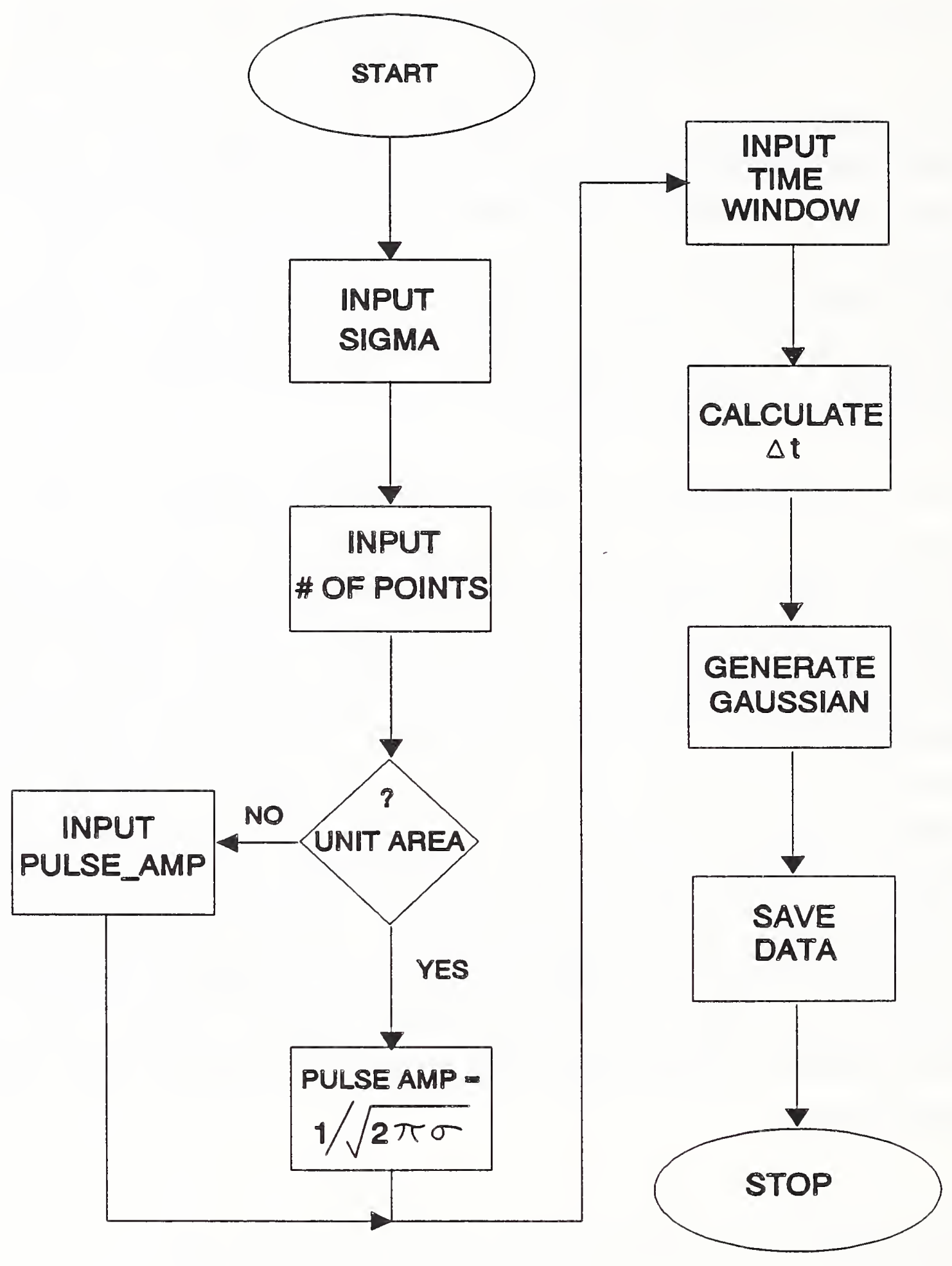

Figure 9. GAUSS flow chart. 


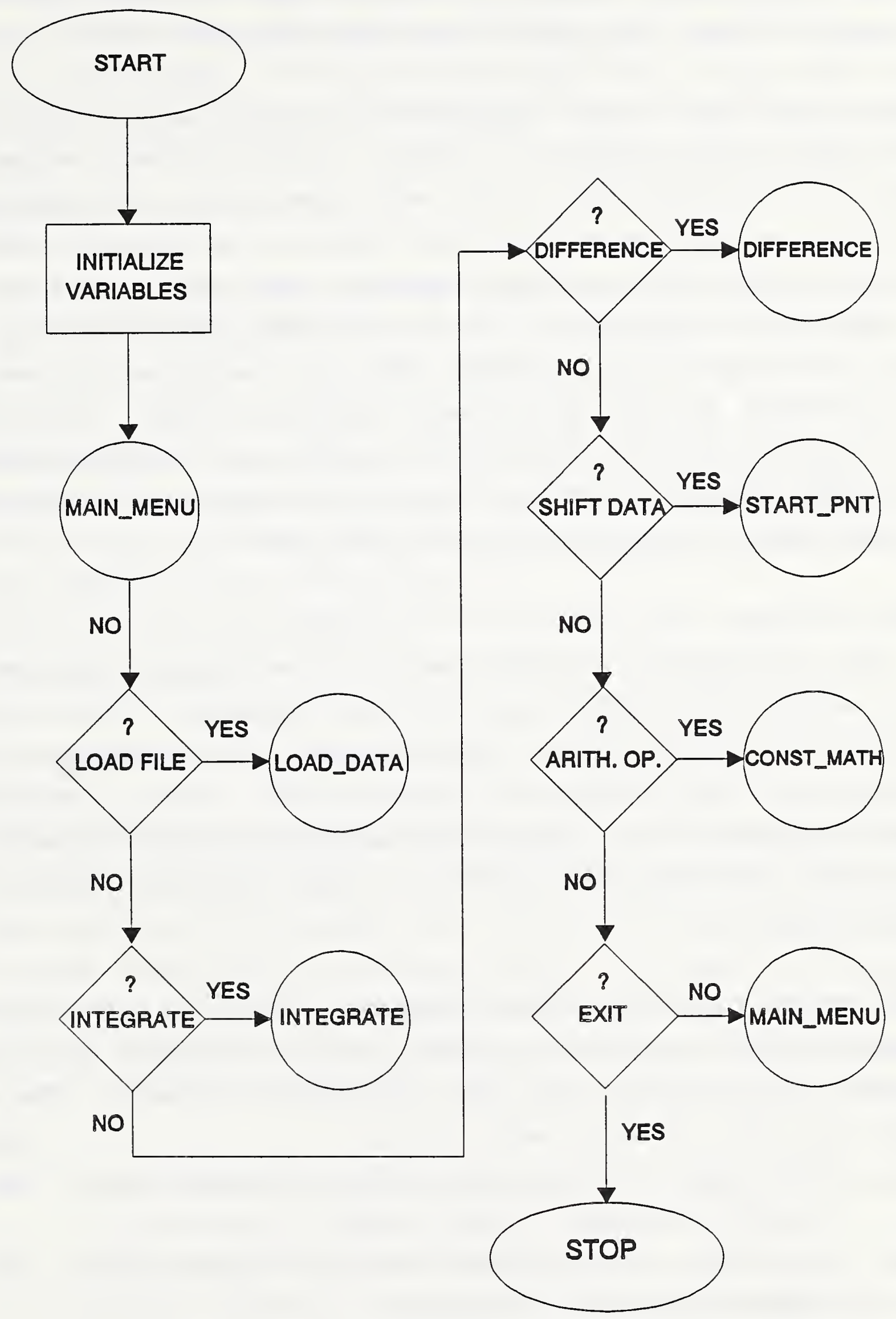

Figure 10. MATH_OPS flow chart. 
acquisition program since it is menu driven. To perform one of the above operations, press the appropriate softkey. To leave the program, select the "Exit" key from the main menu.

The first step after loading and running this program is to use the "Load Data" softkey. If you fail to do this before attempting to perform any of the math operations, the program will display a message telling you there is no file in memory and return you to the main menu so that you may load in a file. After every math operation, the file in the program memory is purged (this does not imply that data have been lost since the data are stored in one of the mass storage units). This means you will have to load a file before beginning a math operation. This program reads and writes files in binary data format and the file structure is compatible with GRAPH_DATA.

The program will perform the corresponding function when you press the "Integrate" or "Differentiate" softkeys. Because the data on which this program operates are discrete, rather than continuous, the integration and differentiation are actually summation and first difference respectively. After completing the selected operation, the program requests a file name and a disk for data storage, the data are saved and the program returns to the main menu.

To access the time shift operation, which is useful for comparing multiple waveforms, press the "Time Shift" key from the main menu. The program will then display a new menu. The options available in this menu are to

1. start the array at the index corresponding to the maximum voltage value;

2. start the array at the index corresponding to the minimum voltage value; or

3. input the starting index number from the keyboard. 
By aligning the waveforms according to a particular waveform feature you will be able to easily discern differences and similarities. After you select the required starting point, the data are rearranged. You are then asked for a file name and disk drive for data storage, the data are stored, and the program returns to the main menu. To see the rearranged waveforms, use GRAPH_DATA.

If you want to add, subtract, multiply, or divide the y-axis data by a constant, press the "+-*/ Y" menu key. You will then have the following softkey menu options: add, subtract, multiply, and divide. After selecting one of the options, input the value for the constant; the program then performs the required operation. Then when asked input the file name and designate a disk drive for data storage, the data are then saved, and the program returns to the main menu.

To leave this program, press the "Exit" key.

4.6.3 Graphics support (GRAPH_DATA)

The AWAMS package includes an up-to-date version of the NIST-written graphics support package, GRAPH_DATA. GRAPH_DATA has been modified to include the ability to draw a histogram plot on the same graph with a waveform plot. In order to include the histogram plot with the waveform plot, you must follow a number of steps in sequence. It is easiest to follow this procedure while you are using GRAPH_DATA and actually performing the key presses.

1. Load the desired file or select it for plotting if it is already in GRAPH_DATA.

2. After selecting the required file, go to the "Auto Scale" option and rescale the graph to this data set. Now is the time to do any manual scaling you require. This is done in the "Edit Background" menu. 
3. Select the "Edit Background" option, press the "Graph Type" softkey, and select the "Histogram" option.

4. Return to the "Edit Data" menu and select the "Data Math" key and then the "User" key (access the "User" key by pressing the shift key on the keyboard and 9 th softkey). When asked, type in "GD_HISTOGRM". This subroutine operates in the same way as the histogram routine in the pulse parameters program, see Ref. [7] for details.

5. Finally, select the curve you just created for plotting and return to the main menu.

It is essential that you select only one file for both plotting and histogram generation as the results will be quite confusing if you do not. At this point, do not re-scale the graph. The scaling of the axes of the histogram graph is dependent on the position of the waveform data graph. Using "Auto Scale" or redefining the graph type will cause an odd looking result. However, you may add labels, change pen color, or any other operation that does not rescale the graph.

GRAPH_DATA has many other features, but these are not directly related to the AWAMS and are therefore not documented here. 
For details on computer, computer peripherals, and oscilloscope operation, see the corresponding operation manuals listed in section A.3.

\subsection{System Changes}

The AWAMS, as delivered, will not need reconfiguring after installation. However, if you decide to change the device bus addresses or change the system hardware, you will also need to change the software to match. Feel free to change bus addresses, user messages, default values, etc. However, please contact the software author(s) before making any changes to the algorithms to maintain consistency between the NIST Automatic Waveform Analysis and Measurement system (AWAMS) and the AWAMS.

\subsection{Acquisition setup}

1. When you acquire a waveform by the computer, the voltage scale seen on the oscilloscope screen is not necessarily the voltage scale acquired. For example, if the signal you measure is nominally $300 \mathrm{mV}$ and the oscilloscope is set at $10 \mathrm{mV}$ per division and the offset is set to center the signal, you will see a clipped waveform on the screen of the oscilloscope, but the acquired waveform will not be clipped. The exception is when the signal exceeds the maximum voltage for the analog-to-digital converter. In that case, the acquired signal will be clipped since this voltage exceeds the maximum oscilloscope capability. WARNING: The maximum safe input voltage into any measurement channel or into the external trigger is $\pm 2 \mathrm{Vdc} \pm$ ac peak. Input voltages that exceed this level may damage or destroy the sampling circuitry. Measurement of signals of this magnitude, or 
because you may destroy the sampling heads of the oscilloscope. See [MUD 1] for details on the oscilloscope specifications. If you want to see a clipped version of the waveform after acquisition, use the manual scaling feature of GRAPH_DATA to display only the desired part of the waveform.

2. Every $4 \mathrm{~ns}$, the oscilloscope will update the clock for the time axis. This event occurs at

$$
16+4 \text { Nns, } N=0,1,2 \ldots
$$

It is essential that your measurement does not include these events. External delay line(s) inserted in the trigger circuit may be required to meet this condition. We do not recommend inserting extra cables in the signal path because of losses in the lines and subsequent distortion of the signal. If you have set up the measurement correctly, this should be no problem for small epochs.

3. When positioning the DUT waveform on the oscilloscope screen, you want the beginning and ending parts of the waveform to visually have nearly zero slope. This condition gives the best results for any subsequent deconvolution. Usually, when the waveform is in this position, the voltage midpoint will be between the first and third graticules.

4. The FFT used by the AWAMS requires $2^{\mathrm{n}}$ points, where $\mathrm{n}$ is an integer. Pick the epoch for measurement so that you can chose the number of points accordingly. For instance, select a $2-n s$ epoch and then choose 2048 points.

5. Increasing the number of points and/or the number of averages in the measurement increases the acquisition time (Section 4.2). It takes approximately 15 minutes to acquire 
(Section 4.2). It takes approximately 15 minutes to acquire a waveform with 1024 points and 2048 averages. Choose the maximum number necessary for each measurement.

6. The acquisition program checks for many error conditions. However, if you mistakenly acquire from the wrong sampling channel, the data will not be what you want. If you get a large number of time calibration factors after using the TCAL feature of the ACQUIRE program, this is likely the problem. Check your setup and try again.

5.3 Oscilloscope and Computer

1. For a complete list of the environmental and operating requirements of the oscilloscope and computer see [MUD $\{1\},\{8\}]$

2. Please wear the grounding wrist strap when operating the oscilloscope. For a complete list of precautions for avoiding electrostatic discharge damage to the oscilloscope, see [MUD $\{1\}]$.

3. The oscilloscope's input connectors are precision $3.5-\mathrm{mm}$ connectors. Although SMA and the precision $3.5-\mathrm{mm}$ connectors appear to mate well, a bad SMA can ruin a good 3.5-mm connector: Please gauge all SMA connectors before coupling to the oscilloscope to verify that they will not harm the input connectors. To increase the lifetime and maintain precision of the connectors, you must use the connectors correctly. Please read [MUD \{1\}] for care and handling. 
6. 0 REFERENCES

1. A.V. Oppenheim and R.W. Schafer, Discrete Time Signal Processing, Prentice-Hall, Englewood Cliffs, NJ, 1989.

2. A.V. Oppenheim and A.S. Willsky, Signals and Systems, Prentice-Hall, Englewood Cliffs, NJ, 1983.

3. Taken from "Minimizing the effects of record truncation discontinuities in waveform deconvolutions" by N.G. Paulter and R.B. Stafford, with permission from N.G. Paulter. Submitted to the Institute of Electrical and Eelectronics Engineers for publication.

4. W.I. Gans and N.S. Nahman, "Continuous and Discrete Fourier Transforms of steplike Waveforms," IEEE Trans. Instrum. Meas., IM-31, p.97, 1982.

5. N.S. Nahman and M.E. Guilluame, "Deconvolution of time domain waveforms in the presence of noise, Nat. Bur. Stand. (U.S.) Tech Note 1047, 1981.

6. "IEEE Standard Pulse Terms and Definitions," IEEE Std 1941977. The Institute of Electrical and Electronics Engineers, Inc., New York, NY, 1977.

7. "IEEE Standard on Pulse Measurement and Analysis by Objective Techniques," ANSI/IEEE std 181-1977. The Institute of Electrical and Electronics Engineers, Inc., New York, NY, 1977 .

8. W. L. Gans, "The measurement and deconvolution of time jitter in equivalent-time waveform samplers," IEEE Trans. Instrum. Meas., IM-32, p. 126, 1983. 


\subsection{GLOSSARY}

bandwidth

the upper frequency limit at which the oscilloscope's voltage response has decreased to 71 percent from its flat frequency response region.

continuous time

all time values, however small the time increment. A continuous-time function is defined for all time.

convolution

mathematically, it is the integration of the multiplication of one function and a time-shifted replica of a second function. A physical example is the interaction of an input waveform and a measurement system which can be described mathematically by a convolution integral.

deconvolution

a mathematical process that allows the reconstruction of one of the two waveforms involved in a convolution. Knowledge of one of the waveforms involved in the convolution is necessary in order to perform a deconvolution.

discrete-time

specific time values, fixed incremental time steps. A discrete-time function is defined only at specific time values.

equivalent-time sampling

sampling of successive points along a periodically repeated waveform so that the sequence of sampled points may be used to reconstruct the waveform. For example, sampling one point from nonidentical locations on each of ten identical repeats of a waveform will allow a 10-point reconstruction of the waveform.

Fourier transform

mathematical transformation of time data into its sinusoidally varying components, or the reverse process.

impulse response

the temporal response of a given device to excitation by a unit-height delta function, or impulse.

interpolate

a method to infer the value of a nonexistent datum, where that datum is located between known or measured data values. 
jitter

random synchronization errors between the trigger. i cos associated event.

sampling window (aperture)

the time duration over which the sampling process occurs.

time aliasing

a distortion in the time representation of a waveform caused by not satisfying the sampling criterion. Basically, the ends of the waveforms overlap and add, thus causing errors in these portions of the waveforms. 
8. OWLEDGEMENTS

.t We hank Larry Tarr for his patience with and support of this project. Additionally, we thank Bill Kissick, Chriss Jones, Bill Gans, and Nancy Keogel sauer for their contributions to this manual; and John Ladbury, Chriss Jones, and Bob Stafford for their inputs, insights, and hours of consultation during the software development. We also thank Galen Koepke for his help with the software. His advice, support, and subroutines were invaluable to this project. 


\section{APPENDIX A}

\section{A.1 Specifications}

The following table lists the various pulse parameters, including their ranges and uncertainties, that are measured with the AWAMS.

\begin{tabular}{|c|c|c||}
\hline PARAMETER & RANGE & $\begin{array}{c}\text { TYPICAL LIMITS OF } \\
\text { UNCERTAINTY }\end{array}$ \\
\hline $\begin{array}{c}\text { Pulse Baseline } \\
\text { (0\% level) }\end{array}$ & $\pm 500 \mathrm{mV}$ & $\pm(0.5 \%+3 \mathrm{mV})$ \\
\hline $\begin{array}{c}\text { Pulse Topline } \\
\text { (100\% level) }\end{array}$ & $\pm 500 \mathrm{mV}$ & $\pm(0.5 \%+3 \mathrm{mV})$ \\
\hline $\begin{array}{c}\text { Pulse Amplitude } \\
\text { Transition Duration } \\
\text { (Rise Time) }\end{array}$ & $10 \mathrm{ps}$ to $100 \mathrm{~ns}$ & $\pm(0.5 \%+3 \mathrm{ps})$ \\
\hline $\begin{array}{c}\text { Pulse Second } \\
\text { Transition Duration } \\
\text { (Fall Time) }\end{array}$ & $10 \mathrm{ps}$ to $100 \mathrm{~ns}$ & $\pm(0.5 \%+3 \mathrm{ps})$ \\
\hline $\begin{array}{c}\text { Pulse Duration } \\
\text { (between 50\% levels) }\end{array}$ & $10 \mathrm{ps}$ to $100 \mathrm{~ns}$ & $\pm(0.5 \%+3 \mathrm{ps})$ \\
\hline
\end{tabular}


The following list provides an inventory of the hardware presently provided with the AWAMS. A brief description of these components is also given. However, for detailed information, please see the manufacturer users's manuals listed in Sec. A.3. We use trade names to specify the equipment used in this system and no endorsement by the National Institute of standards and Technology is implied. Similar products by other manufacturers may work as well or better.

\section{Digitizing oscilloscope}

The HP Model $54120 \mathrm{~T}$ digitizing oscilloscope provides $20 \mathrm{GHz}$ bandwidth, full IEEE 488 programmability, time-domain reflectometry capability, and waveform arithmetic operations.

\section{Computer/instrument controller}

The HP 9000/300 Microcomputer operates at $16.6 \mathrm{MHz}$, has 8 megabytes of RAM, and IEEE 488, LAN, and RS232C interfaces.

\section{Disk Drives}

The HP 9127A Disk Drive uses 5.25-inch diameter, magnetic storage, flexible disks.

The HP $9122 \mathrm{C}$ has 2-megabyte data storage capacity and uses double-sided, 3.5-inch diameter floppy disks.

The HP 7957B is a hard disk drive unit and has 81-megabyte of data storage capacity.

\section{Printers}

The HP ThinkJet and HP PaintJet printers are used with the AWAMS . 


\section{A.3 Manufacturers' Users' Documentation}

The following is a list of the manufacturers' users' documentation. Familiararity with these manuals is essential to the proper operation of the AWAMS.

1. "HP 54120 User Documentation (Installation/Operation Manual)," HP Manual Part Number 98613-90000, Hewlett-Packard Company, U.S.A., 1987.

2. "HP 54121T Digitizing Oscilloscope Programming Reference," HP Manual Part Number 54121-90907, Hewlett-Packard Company, U.S.A., 1989.

3. "Using the BASIC 5.0/5.1 System, HP 9000 series 200/300," HP Part Number 98613-90000, Hewlett-Packard, U.S.A., 1988.

4. "BASIC 5.0/5.1 Interfacing Techniques, Vol. 1: General Topics, HP 9000 Series $200 / 300$ Computers," HP Part Number 98613-90022, Hewlett-Packard Company, U.S.A., 1987.

5. "Basic Language Reference Volume $1 \mathrm{~A}-\mathrm{N}$," HP Part Number 98613-90052, Hewlett-Packard Company, U.S.A., 1989.

6. "Basic Language Reference Volume $1 \mathrm{O}-\mathrm{Z}$," HP Part Number 98613-90052, Hewlett-Packard Company, U.S.A., 1989.

7. "Basic 5.0/5.1 Programming Techniques Volume 1 : General Topics," HP Part Number 98613-90813, Hewlett-Packard Company, U.S.A., 1988.

8. "Installation Reference, HP 9000 Series 300 Computers," HP Part Number 9856-90000, Hewlett-Packard Company, U.S.A., 1988 .

9. "Getting started with Your HP 9127A Disc Drive," HP Manual Part Number 09127-90000, Hewlett-Packard Company, U.S.A., 1989.

10. "Getting started with Your HP 9122C Disc Drive," HP Manual Part Number 09122-90901, Edition 2, Hewlett-Packard Company, U.S.A., 1988 .

11. "HP 7957B, HP 7958B, and HP 7959B Disc Drives," HP Manual Part Number 07959-90901, Hewlett-Packard Company, U.S.A., 1988 . 
12. "Personal Printer ThinkJet, Owner's Manual," HP Manual Part Number 02225-90031, Hewlett-Packard Company, Singapore, 1987. 
A. 4 Measurement Setup and Example Procedures

A.4.1 General

Please read the section titled "Handling and care of the Precision Connectors" [MUD\{1\}] before using the oscilloscope.

1. Clean all connectors on the oscilloscope, the DUT, the voltage calibration standard, the time calibration standard, and any required adapters, filters, and cables.

2. Connect the 3.5-mm shorts to all of the oscilloscope channels and the trigger input. Run the vertical calibration utility of the oscilloscope [MUD\{1\}]。

3. You may use any of the four sampling channels for the measurement; this is represented as "Chan $X$ " in Figs. $A 1$ and $A 2$. The asterisks in Figs. $A 1$ and $A 2$ indicate the equipment supplied as part of the AWAMS.

4. The solid lines in Figs. A1 and A2 represent the DUT connections, the dotted lines the time calibration standard connections, and the dashed lines the voltage calibration connections. The test equipment marked with an "*" in Figs. A1 and $A 2$ are the only pieces that are provided as part of the AWAMS. You must provide all other test equipment.

5. Record the time, the date, and the room temperature and humidity before beginning data acquisition. These values should be recorded periodically throughout the test.

A.4.2 Pulse generator, procedure 1

This procedure is used for measuring the Tektronix S-52 pulse generator output; this pulser will be referred to as P1. NIST has received many requests to the measure the output of P1 and, consequently, has developed procedures for measuring its 


\section{CALIBRATION SETUP FOR P1}

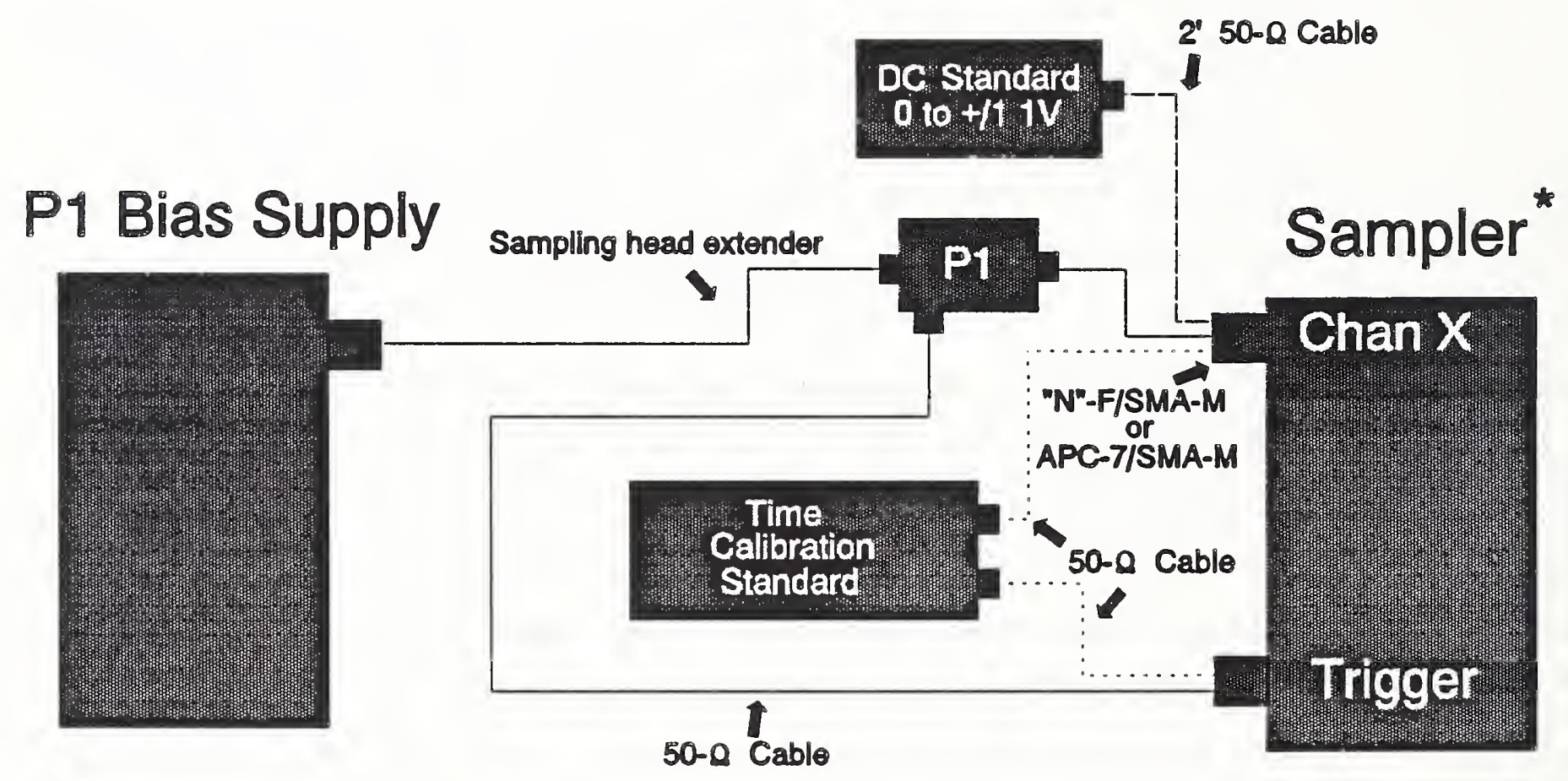

Figure A1. Pulse generator measurement setup 1. 


\section{CALIBRATION SETUP FOR P2}

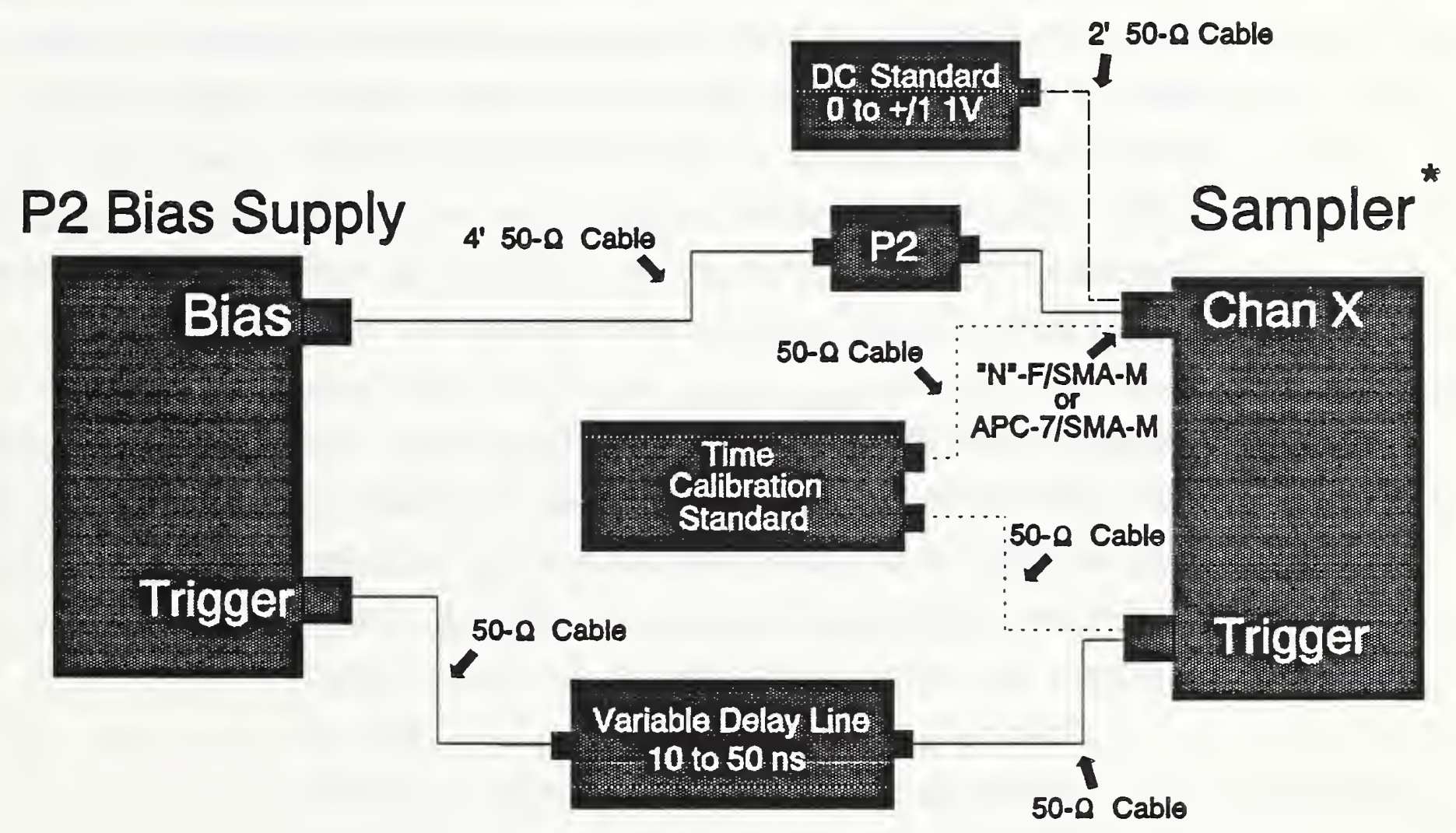

Figure A2. Pulse generator measurement setup 2. 
output. However, this does not imply any preference, by NIST, for this product.

1. Load and run the ACQUIRE program. You should determine and set the acquisition parameter values for each menu before acquiring any data. Procedures for determining these values follow. Once the menus have been set up, you will not need to redo them. You will acquire nine or more data sets. A data set consists of one DUT waveform, one time-calibration waveform, and one group (see VCAL description) of voltage-calibration factors. You may acquire these three components in any order but each set must be completed before beginning another. once you choose an order of acquisition, maintain that order throughout the test. For example, first acquire the time-calibration data, then the voltage-calibration data, and finally, the DUT data.

2. Connect the P1 as shown in Fig. A1. Press the waveform menu key and select the manual setup option. View the DUT waveform on the oscilloscope screen and manually position the waveform as required.

Typical P1 settings are:

Channel 4;

Volts per Division: $50 \mathrm{mV}$;

Offset: $215.00 \mathrm{mv}$;

Attenuation: 1;

Time per Division: 200 ps;

Delay: 81.275 ns;

Delay Reference: center;

Points: 1024 ;

Averages: 2048 ;

Trigger Level: $300 \mathrm{mv}$;

Trigger slope: positive (this is not one of the ACQUIRE program menu selections). 
These are typical settings; yours may be different. Write down the oscilloscope settings that you use. Make certain that the time window does not have one of the 4-ns boundaries described in sec. 5.2. If the epoch does have a 4-ns boundary, you may need to use an external delay line to reposition the waveform. Check the minimum and the maximum voltages that span the waveform's voltage range; this will be used in the subsequent voltage calibration. Once the oscilloscope has been setup, return to the main menu.

3. Connect your time calibration standard as shown in Fig. A1. Press the TCAL menu key and then the manual setup key. View the waveform and establish the required oscilloscope settings. The first mid-point crossing of the time calibration waveform should occur before the location of the first transition of the pulse waveform. The time/div and the delay settings of the oscilloscope must be the same as the those for DUT. Ose external delay lines to position the calibration waveform. You may change the volts per division and offset settings as needed. Typical time calibration settings are:

Frequency: $5.0 \times 10^{9} \mathrm{~Hz}$;

Volts per division: $1.0 \mathrm{mV}$;

offset: $0.0 \mathrm{mV}$ (as needed to center the waveform);

Number of sliding average point pairs: 7 ;

Number of least squares points: 20 ;

slope: positive;

Save the waveform: no;

Time calibration signal trigger level: $150 \mathrm{mV}$. Record the time calibration settings. Return to the main menu.

4. Connect your DC standard as shown in Fig. A1. Press the VCAL key and then the manual setup key. The volts 
per division and offset values must be the same as those for the DUT. Other settings will invalidate the voltage-calibration data. Determine the number of voltage steps and the voltage increment needed to span the waveforms voltage range (previously determined). Typical settings are:

Voltage intervals: 7 ;

Averages: 128 ;

Minimum or starting voltage: $0.05 \mathrm{mV}$;

Voltage step size: $0.05 \mathrm{mV}$.

Record the voltage calibration settings.

5. Acquire and record the nine sets of data.

6. When you have collected nine data sets, exit the ACQUIRE program.

7. Measure the sigma for the jitter impulse response. The procedure for measuring the jitter is outlined in sec. A.4.4 of this Manual.

8. Create the jitter impulse response using the GAUSS program.

9. Calibrate all nine waveforms using the FIXACQ program and the corresponding calibration data.

10. Deconvolve the jitter function from all nine calibrated waveforms using the DECON_NIST program.

11. Deconvolve the system impulse response, SYS_RESP, from all nine calibrated, jitter-deconvolved waveforms using the DECON_NIST program. SYS_RESP is the name of the file that contains the system impulse response for the oscilloscope.

12. Run the PULS_PARAMS program on all nine of the calibrated, jitter-deconvolved, system-deconvolved waveforms. These are the corrected waveforms.

13. Calculate the mean and standard deviation of the following parameters: pulse amplitude (not the peak-topeak value), 10-90\% transition duration, 20-80\% transition duration, percentage overshoot, and the 
percentage undershoot. (Do these calculations for all of the pulse parameter data that you have generated.)

14. Determine which of the nine corrected waveforms has parameter values closest to the mean values calculated above; this is the representative waveform. Use this representative waveform for the report plots.

15. Plot the representative waveform.

16. Calculate the uncertainties in the representative waveform's pulse parameters. You may use the limits listed in Sec. A.1 for these calculations.

17. Generate the test report.

\section{A.4.3 Pulse generator, procedure 2}

This procedure is used for measuring the Hewlett-Packard $1106 \mathrm{~A}$ and 1106B pulse generator outputs; these pulsers will be referred to as P2. NIST has received many requests to the measure the output of P2 and, consequently, has developed procedures for measuring its output. However, this does not imply any preference, by NIST, for this product.

1. Load and run the ACQUIRE program. You should determine and set the acquisition parameter values for each menu before acquiring any data. Procedures for determining these values follow. Once the menus have been set up, you will not need to redo them. You will acquire nine or more data sets. A data set consists of one DUT waveform, one time-calibration waveform, and one group of voltage-calibration factors. You may acquire these three components in any order each set must completed before beginning another. Once you choose an order of acquisition, maintain that routine throughout the test. For example, first acquire the time-calibration data, then the voltage-calibration data, and finally, the DUT data. 
2. Connect $\mathrm{P} 2$ as shown in Fig. A2. Press the waveform menu key and then select the manual setup option. View the DUT waveform on the oscilloscope screen and manually position the waveform as required. Typical P2 settings are:

Channel 4;

Volts per division: $50 \mathrm{mV}$;

Offset: $235.00 \mathrm{mV}$ (as required to center the waveform);

Attenuation: 1;

Time per division: 200 ps;

Delay: 33.100 ns;

Delay Reference: center;

Points: 1024 ;

Averages: 2048 ;

Trigger Level: $400 \mathrm{mV}$;

Trigger slope: positive (this is not one of the ACQUIRE program menu selections).

These are typical settings; yours may be different. Write down the oscilloscope settings that you use. Make certain that the time window does not have one of the 4-ns boundaries described in Sec. 5.2. If the epoch does have a 4-ns boundary, you may need to use an external delay line to reposition the waveform. Check the minimum and the maximum voltages that span the waveform's voltage range; this will be used in the subsequent voltage calibration. Once the oscilloscope has been set up, return to the main menu.

3. Connect your time calibration standard as shown in Fig. A2. Press the TCAL menu key and then the manual setup key. View the waveform and establish the required oscilloscope settings. The first mid-point crossing of the time calibration waveform should occur before the 
location of the first transition of the pulse waveform. The time per division and the delay settings of the oscilloscope must be the same as the those for DUT. Use external delay lines to position the calibration waveform. You may change the volts per division and offset settings as needed. Typical time calibration settings are:

Frequency: $5.0 \times 10^{9} \mathrm{~Hz}$;

Volts per division: $1.0 \mathrm{mV}$;

offset: $0.0 \mathrm{mV}$ (as needed to center the waveform);

Number of sliding average point pairs: 7;

Number of least squares points: 20;

slope: positive;

Save the waveform: no;

Time calibration signal trigger level: $150 \mathrm{mV}$. Record the time calibration settings. Return to the main menu.

4. Connect your DC standard as shown in Fig. A2. Press the VCAL key then the manual setup key. The volts per division and offset values must be the same as those for the DUT. Other settings will invalidate the voltage calibration data. Determine the number of voltage steps and the voltage increment needed to span the waveforms voltage range (previously determined). Typical settings are:

Voltage intervals: 7 ;

Averages: 128 ;

Minimum or starting voltage: $0.05 \mathrm{mV}$;

Voltage step size: $0.05 \mathrm{mv}$.

Record the voltage calibration settings.

5. Acquire and record the nine sets of data.

6. When you have collected nine data sets, exit the ACQUIRE program. 
7. Measure the sigma for the jitter impulse response. The procedure for measuring the jitter is outlined in Sec. A.4.4 of this Manual.

8. Create the jitter impulse response using the GAUSS program.

9. Calibrate all nine waveforms using the FIXACQ program and the corresponding calibration data.

10. Deconvolve the jitter function from all nine calibrated waveforms using the DECON_NIST program.

11. Deconvolve the system impulse response, SYS_RESP, from all nine calibrated, jitter-deconvolved waveforms using the DECON_NIST program. SYS_RESP is the name of the file that contains the system impulse response for the oscilloscope.

12. Run the PULS_PARAMS program on all nine of the calibrated, jitter-deconvolved, system-deconvolved waveforms. These are the corrected waveforms.

13. Calculate the mean and standard deviation of the following parameters: pulse amplitude (not the peak-topeak value), 10-90\% transition duration, 20-80\% transition duration, percentage overshoot, and the percentage undershoot. (Do these calculations for all of the pulse parameter data that you have generated.)

14. Determine which of the nine corrected waveforms has parameter values closest to the mean values calculated above; this is the representative. Use this representative measurement waveform for the report plots.

15. Plot the representative waveform.

16. Calculate the uncertainties in the representative waveform's pulse parameters. You may use the limits listed in Sec. A.I for these calculations.

17. Generate the test report. 


\section{A.4.4 Jitter Measurement}

1. Use the same oscilloscope settings as those for the DUT. Record the time and the room temperature and relative humidity.

2. Select the "Delta V" oscilloscope menu key. Turn the voltage markers on and select the measurement channel for both markers.

3. Press the "Auto Level set" key. Select the "Preset Levels = 50\%-50\%"

4. Record the displayed 50\% amplitude value.

5. Select the "Histogram" oscilloscope menu key, "Time Histogram," and then "Window."

6. Using the oscilloscope knob, set marker number 2 to the $50 \%$ voltage value.

7. Use the oscilloscope knob to set marker 1 to one voltage increment above the marker-2 voltage position. This is usually approximately $1.5 \mathrm{mV}$. Record the voltage values of each marker.

8. Select the "Acquire" oscilloscope key and then set the "Number of Samples" to 500 .

9. Press the "start Acquire" key.

10. When the oscilloscope has finished the measurement, press the "Results" key and then the "Sigma" key.

11. Record the displayed value of sigma.

12. Repeat steps 8 through 11 nine times.

13. Calculate the mean and standard deviation of the nine recorded values. Use the mean value for the input to the GAUSS program. 

APPENDIX B (Software source code listings)

This appendix contains the source code listing of the programs ACQUIRE, DECON_NIST, FIXACQ, PULS_PARAMS, GAUSS, GD_HISTOGRAM, MATH_OPS, text_out that are used in the AWAMS. 
$100 !$ RE-STORE "ACQUIRE:, 1400"

102

104

106

108

110

112

194

116

118

120

122

124

126

128

130

132

134

136

138

140

142

144

146 Date

148

150

152

154

156

158

160

162

164

166

168

170

172

174

176

178

180

182

184

186

188

190

192

194

196

198

200

202

204

206

208

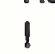


212 GOSUB Init_mnemonics

214 GOSUB Load_averages

222 Exit: ! !!!!!!!!!!!!!!!!!!!!!!!!!!!!!!!!!!!!!!!!!!!!!!!!!!!!!!!!!

266 !nit_variables:!

CLEAR SCREEN LOCAL 707 
392 !nit graphics:!

394

396

398

400

402

404

406

408

410

412

414

4961

418 !

420 Start_vals: $\quad 1$ set-up default values

422

424

426

428

430

432

434

436

Beep_flag $=0$

Stp $=0$

Info_screen $\$=" n$ "

Local_prty $=$ Intr_prty

Paused $=1.3$

Mode $\$=$ "TRIGGERED"

$!$

RETURN

I
Ivalue for the initial run. lotherwise stored in COM/mnu, COM/Scope,

END IF ICOM/Tcal_vals, and COM/Ncal_vals 


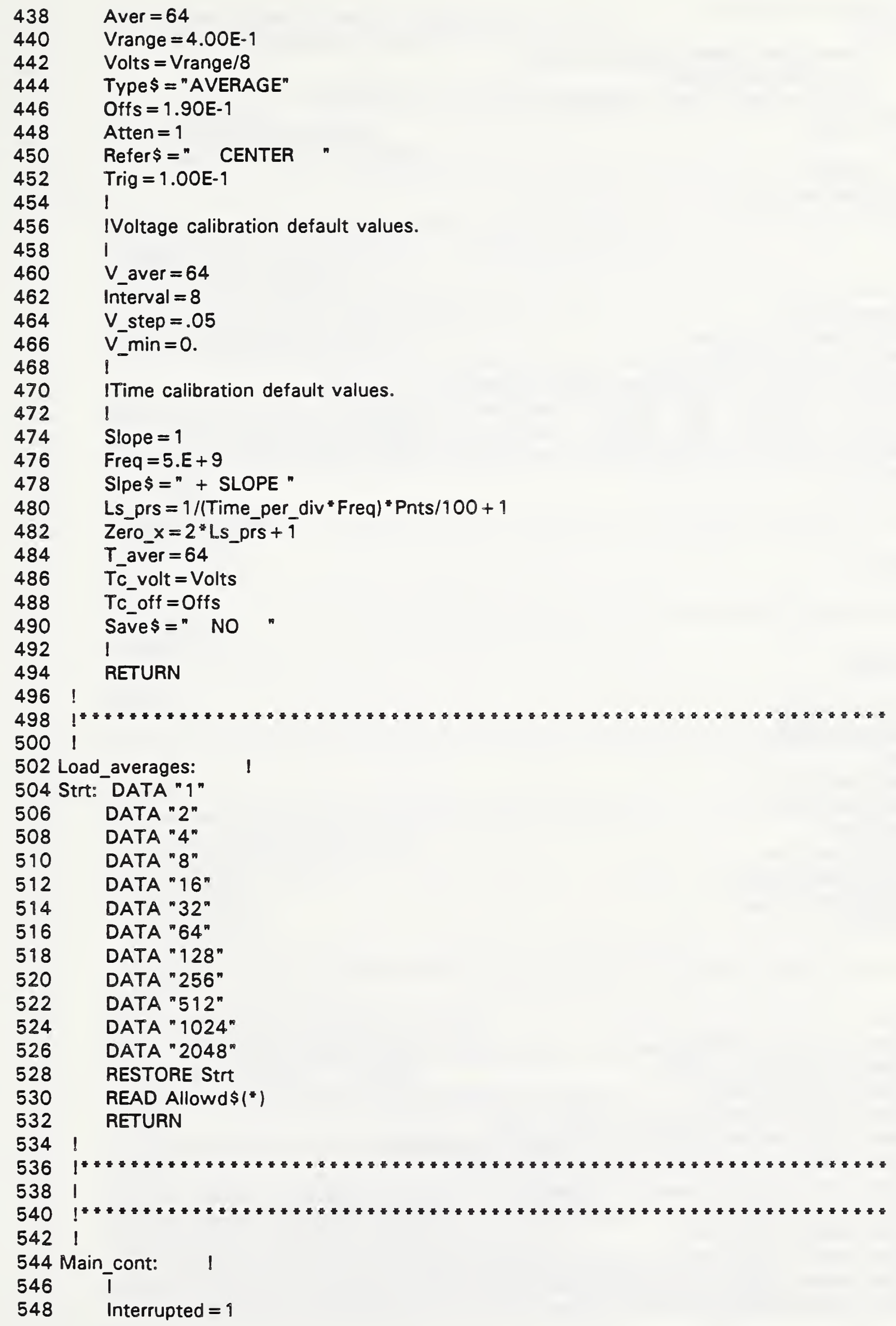




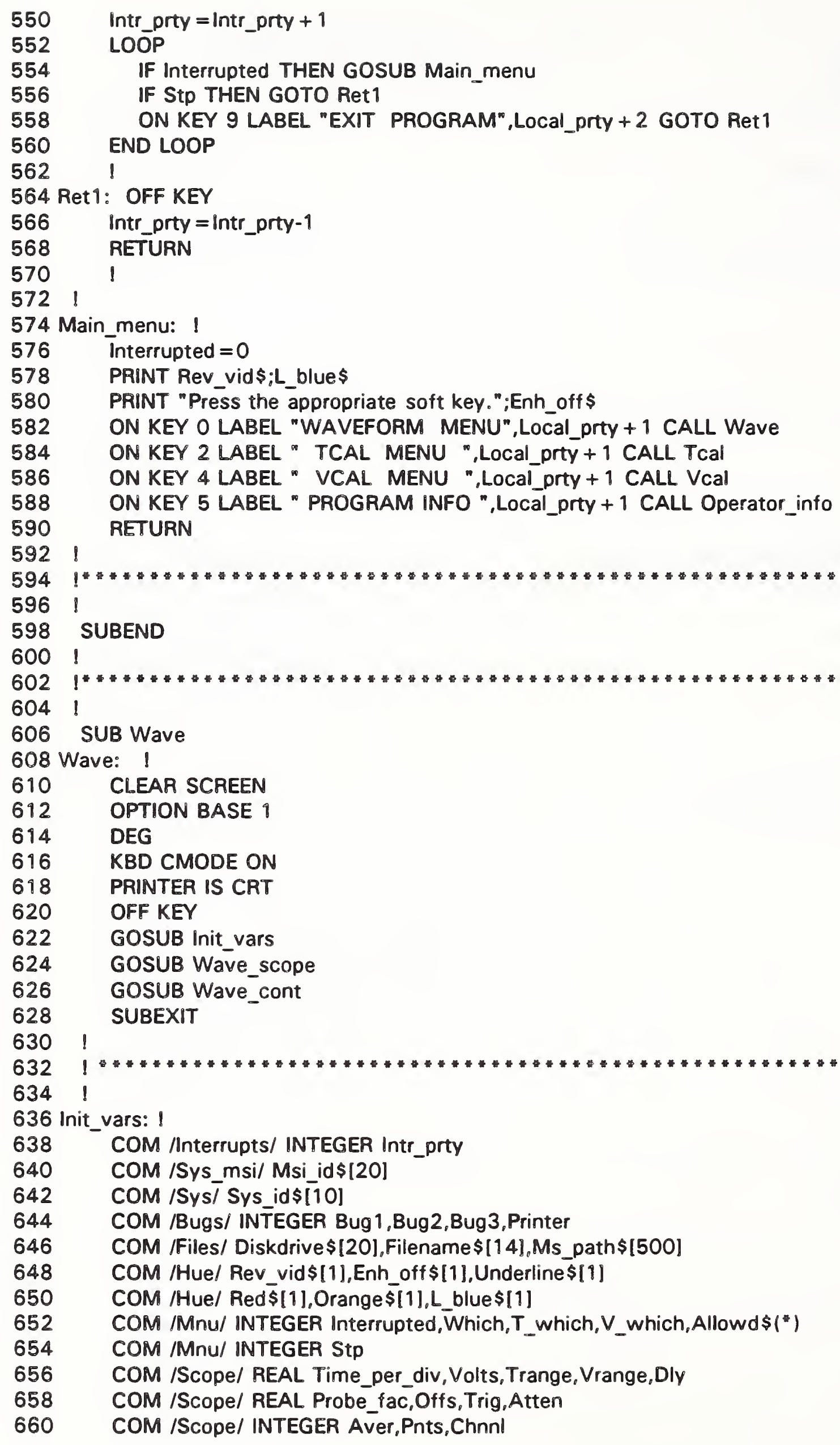


662

664

666

668

670

672

674

676

678

680

682

684

686

688

690

692

694

696

698

700

702

704

706

708

710

712

714

716

718

720

722

724

726

728 Wave

730

732

734

736

738

740

742

744

746

748

750

752

754

756

758

760

762

764

766

768

770

772 $!$

!*

COM /Scope/ Type\$[30],Refer\$[14],@Scope,Mode\$[30]

COM /Slect/ INTEGER Last1, Tlast, Vlast

1

1

DIM Version\$[80]

DIM Test\$[160],Data_id\$[40]

DIM Choice\$[15],Ch\$[1],Step\$[50] 1

1

INTEGER Co_ords $(12,4)$,Lwrlftx,Lwrlfty,Upperrtx,Upperrtty

INTEGER Num_of_choices, What (1)

INTEGER I,J,Pen, $\overline{\text { Knnobcount }}$

INTEGER Error_flag,Beep_flag,Local_prty,Valid,Datacount

INTEGER Filesize,Baddata, Endpoint, Print_val

INTEGER Yref,Temp,Item_cnt,Err_flg

!

!

REAL Data entered,Data set count

REAL Yinc, Yor,Rtemp,Waveform(32767)

DIM Dp\$[80]

DIM T\$[52]

Local_prty = Intr_prty

Ftype $\$=$ "BDAT"

Filesize $=500$

Last $1=0$

PEN 1

Beep_flag $=0$

Paused $=1.5$

!

RETURN

I

IThis returns the waveform menu/scope values to those in

I the common block called Scope.

CALL Scope_init(Err_flg)

IF Err_flg THEN

Wave_intrpt $=1$

Interrupted $=1$

Err_flg $=0$

SUBEXIT

END IF

CLEAR 707 Iclears the HPIB to the scope

OUTPUT @Scope; :TRIGGER:LEVEL "\&VAL\$(Trig)

IF Refer $\$=$ " CENTER "THEN

OUTPUT @Scope;"TIMEBASE:REFERENCE CENTER"

ELSE

OUTPUT @Scope;"TIMEBASE:REFERENCE LEFT"

END IF

OUTPUT @Scope;":TIMEBASE:DELAY "\&VAL\$(Dly)

OUTPUT @Scope;":TIMEBASE:RANGE "\&VAL\$(Trange)

OUTPUT @Scope;"VIEW CHANNEL" \&VAL\$(Chnnl)

OUTPUT @Scope;":CHANNEL"\&VAL\$(Chnnl)\&":OFFSET "\&VAL\$(Offs)

OUTPUT @Scope;":CHANNEL" \&VAL\$(Chnnl)\&":RANGE "\&VAL\$(Vrange)

OUTPUT @Scope;":ACQUIRE:COUNT "\&VAL\$(Aver) 


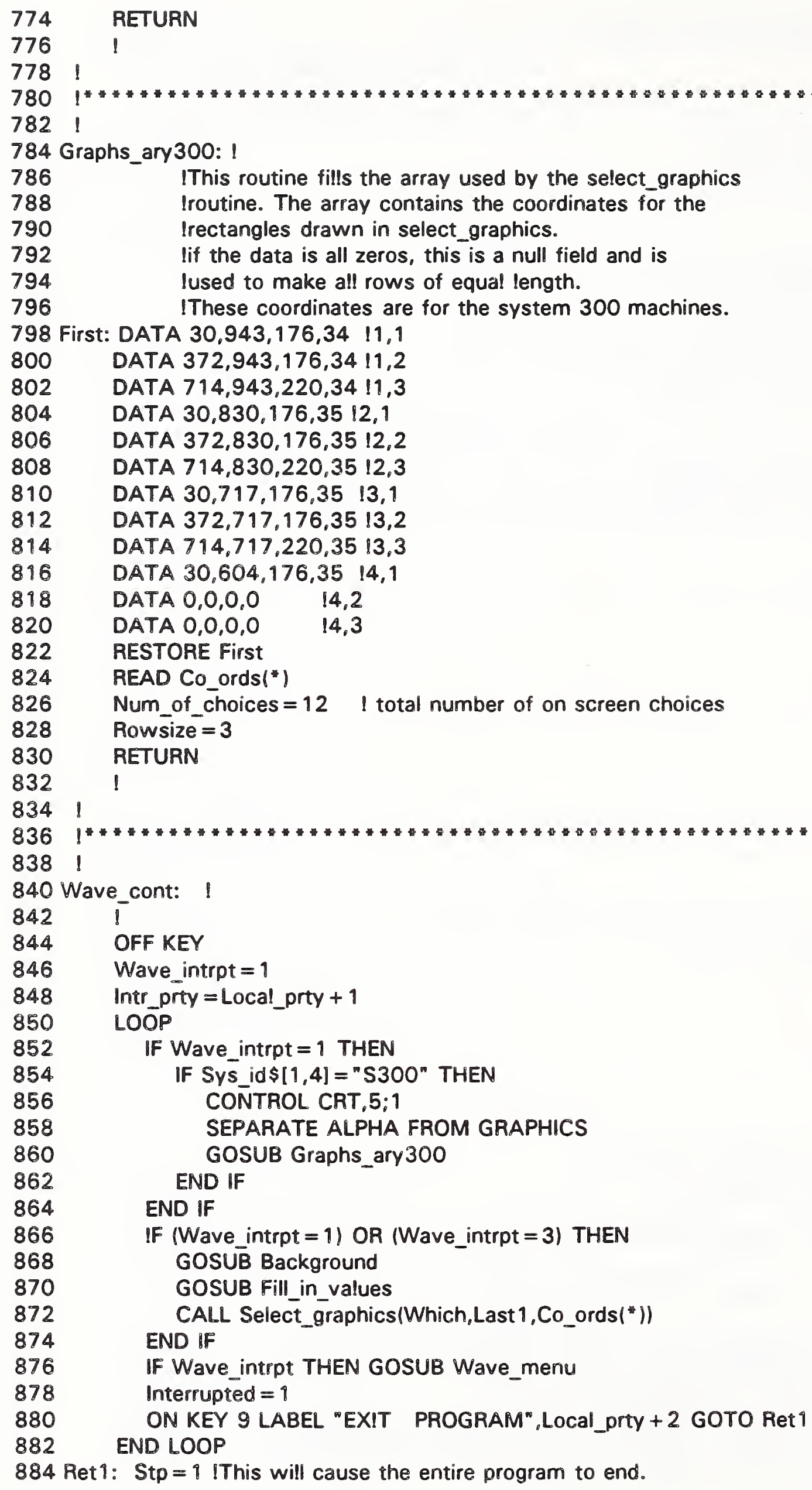

884 Ret $1:$ Stp $=1$ IThis will cause the entire program to end. 
$886 \quad$ Interrupted $=0$

888 Ret2: OFF KEY IThis will exit only this subprogram.

890 CLEAR SCREEN

892 Intr_prty = Local_prty

894 RETURN

$896 \quad$

898

9001

902 Wave_menu: 1

904 Wave_intrpt $=0$

906 OFF KEY

908 OFF KBD

910 OFF KNOB

912 Knobcount $=0$

914 DISP Orange $\$$;"DUT MENU"

916 ON KBD,Local_prty + 1 GOSUB Process_kbd

918 ON KNOB .01, Local_prty + 1 GOSUB Move_pointer

920 ON KEY 1 LABEL "ACOUIRE DATA ",Local_prty + 9 GOSUB Take_data

922 ON KEY 7 LABEL "MANUAL SETUP" "Local_prty + 1 GOSUB Manual_set

924

926

928

930

932

934

936

938

940

942

944

946 


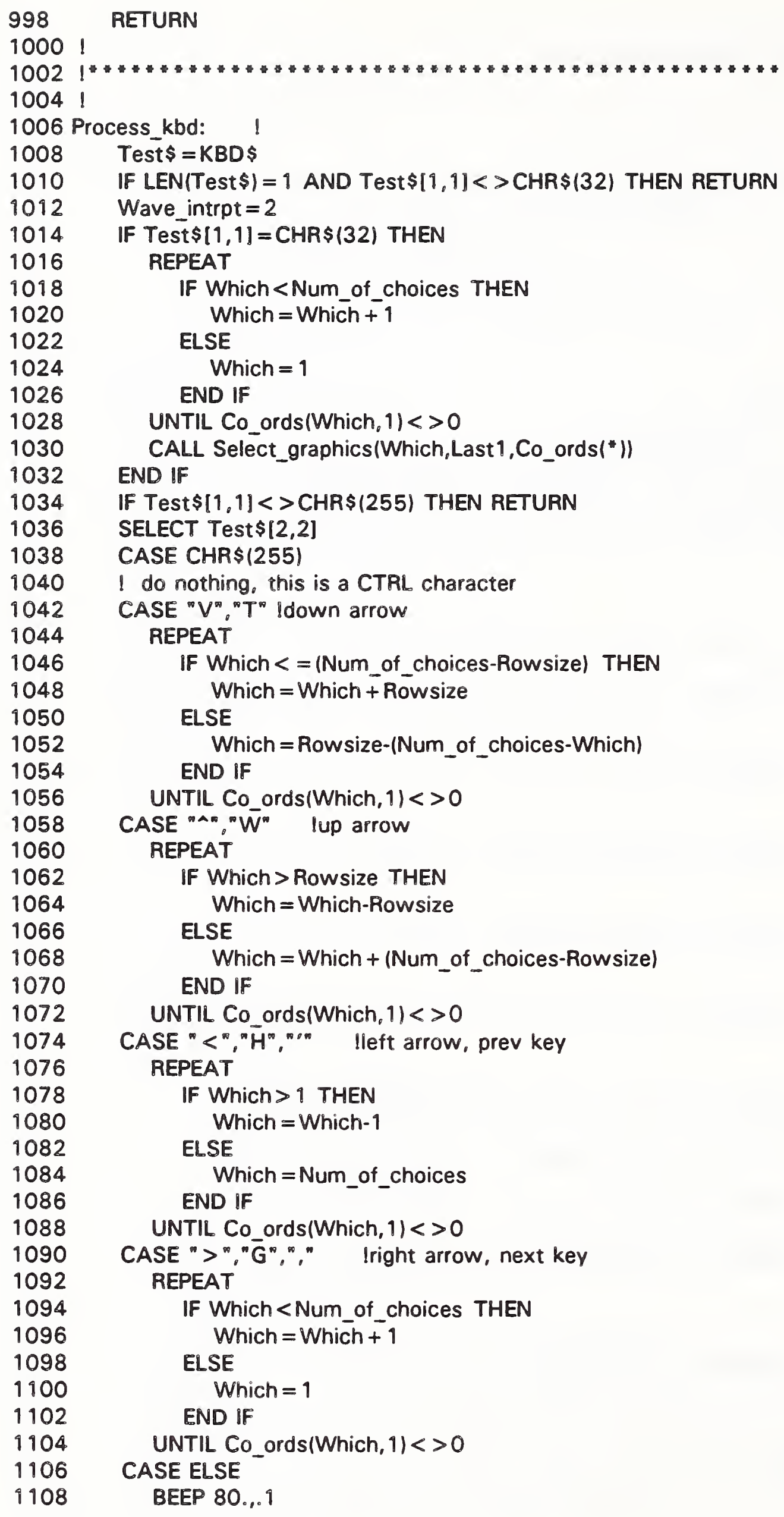$$
\text { ELSE }
$$$$
\text { Which }=\text { Which }+1
$$$$
\text { Which }=1
$$

END IF

UNTIL Co_ords(Which, 1$)<>0$

CALL Select_graphics (Which,Last 1,Co_ords/*)।

\section{END IF}

IF Test $\$[1,1]<>$ CHR\$(255) THEN RETURN

\section{SELECT Test $\$[2,2]$}

\section{CASE CHR\$(255)}

$I$ do nothing, this is a CTRL character

CASE " $V "{ }^{*}{ }^{*} T^{"}$ ldown aprow

REPEAT

IF Which $<=$ (Num_of_choices-Rowsize) THEN

$$
\text { Which }=\text { Which }+ \text { Rowsize }
$$

ELSE

$$
\text { END IF }
$$

UNTIL Co_ords (Which, 1$)<>0$

CASE ${ }^{n A \infty},{ }^{\circ} W^{\infty}$ lup aprow

REPEAT

IF Which > Rowsize THEN

Which $=$ Which-Rowsize

ELSE

Which $=$ Which $+($ Num_of_choices-Rowsize $)$

END IF

UNTIL Co_ords(Which, 1$)<>0$

CASE ${ }^{"}<{ }^{\infty},{ }^{n} H^{\infty}$, "

REPEAT

IF Which $>1$ THEN

Which $=$ Which -1

ELSE

Which = Num_of_choices

END IF

UNTIL Co_ords(Which, 1$)<>0$

CASE $">{ }^{\infty}, \bar{G}^{\infty},{ }^{\infty}, " \quad$ Iright arrow, next key REPEAT

IF Which < Num_of_choices THEN

Which $=$ Which +1

ELSE

Which $=1$

END IF

UNTIL Co_ords(Which, 1 ) $<>0$

CASE ELSE

BEEP 80... 1 
1122 Move_pointer:

1124 Knobcount $=$ Knobcount + KNOBX-KNOBY

1126 IF ABS(Knobcount) $<15$ THEN RETURN

1128

1130

1132

1134

1136

1138

1140

1142

1144

1146

1148

1150

1152

$1154 !$

1156

1158 !

1160 Background

1162

1164

1166

1168

1170

1172

1174

1176

1178

1180

1182

1184

1186

1188

1190

1192

1194

$1996 !$

Wave_intrpt $=2$

REPEĀT

IF Knobcount $>0$ THEN

$$
\text { Which }=\text { Which }+1
$$

$$
\text { ELSE }
$$

Which $=$ Which -1

END IF

IF Which $<1$ THEN Which $=$ Num of choices

IF Which > Num_of_choices THEN Which $=1$

UNTIL Co_ords(Which, 1 ) $<>0$

CALL Select_graphics(Which,Last1,Co_ords ("))

Knobcount $=0$

RETURN

1198

1200 !

1202 Fill_in_values: !

1204 GOSUB Print_chnnl

1206

1208

1210

1212

1214

1216

1218

CLEAR SCREEN

IF SYS id $\$[1,4]={ }^{\text {" }} \$ 300$ " THEN

MERGE ALPHA WITH GRAPHICSIThis gives back the colors to the alpha

END IF Iplane.

PRINT TABXY $(1,1) ;$ Rev_vid\$;L_blue\$;" CHANNEL ";Enh_off $\$$

PRINT TABXY $(1,4) ;$ Rev_vid\$;L_blue $\$ ; "$ VOLTS/DIV (v) ";Enh_off $\$$

PRINT TABXY(1,7);Rev_vid\$;L_blues;" OFFSET (v) ";Enh_off\$;

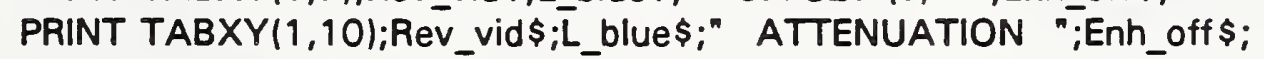

PRINT TABXY $(30,1)$;Rev_vid\$;L_blues;" TIME/DIV (s) ";Enh_off\$;

PRINT TABXY $(30,4) ;$ Rev_vid $\$$;_blues;" DELAY (s) ";Enh_off\$;

PRINT TABXY $(30,7) ;$ Rev_vid\$;L_blue\$;" DELAY REFER. ";Enh_off $\$ ;$

PRINT TABXY $(59,1) ;$ Rev_vid\$;L_blue $\$ ; "$ \#OF AVERAGES ";Enh_off\$;

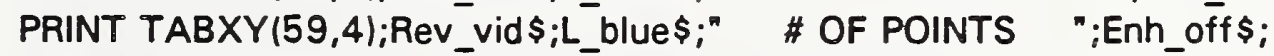

PRINT TABXY $(59,7) ;$ Rev_vids;L_blues;" TRIGGER LEVEL (v) ";Enh_off\$;

RETURN

1220

GOSUB Print_time

GOSUB Print_volts

GOSUB Print_pnts

GOSUB Print_ave

GOSUB Print_delay

GOSUB Print_ref

GOSUB Print_offset

GOSUB Print_trig_lev 
$1246 !$

1248 Choose_average: !

1250

1252

1254

1256

1258

1260

1262

1264

1266

1268

1270

1272

1274

1276

1278

1280

1282

1284

1286

1288

1290

1292

1294

1296

1298

1300

1302

1304 Chnnl error: !

1306

1308

1310

1312

1314

1316

1318

1320

1322

1324

1326

1328

1330

1332
GOSUB Print_atten

RETURN
OFF KEY

OFF KNOB

OFF KBD

CLEAR SCREEN

GCLEAR

PRINT L_blue

Intr_prty $=$ Intr_prty +3

Intr_prty = Intr_prty-3

END IF

Wave_intrpt $=3$

I

RETURN

Print_ave:

RETURN

1

\section{BEEP}

WAIT Paused

DISP "Try again ..."

Sel_chn: !

Test $\$="$ "

Temp $=$ VAL(Test $\$$ )

OFF ERROR

Chnnl $=$ Temp

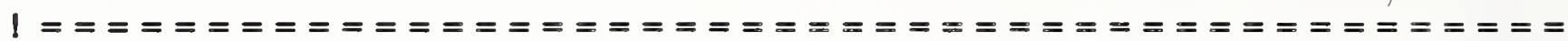

l I Data input subroutines

I

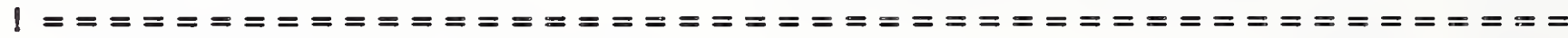

$\mathrm{Dp} \$=$ "Select Average "

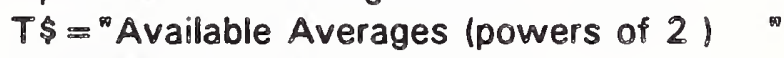

CALL Menu_scroll(Dp\$,T\$,Allowd $\$(*), 12,1$, What $(*)$

IF What $(1)<>0$ THEN \& Aborted

Aver $=$ VAL(Allowd $\$($ What (1))

OUTPUT @Scope;" :ACQUIRE:TYPE AVERAGE

OUTPUT @SCOpe;":ACQUIRE:COUNT "\&VAL\$(Aver)

PRINT TABXY(64,2); Orange\$;

CALL Auto_format(Aver"1.0)

DISP "Input out of range of disallowed value."

ON ERROR GOTO Chnnl_error

INPUT "Enter the acquisition channel number $(1-4) .0$ "

IF LEN(Test\$) $<1$ THEN RETURN

IF (Temp<1) OR (Temp > 4) THEN GOTO Chnnl_error

OUTPUT @Scope;"BLANK CHANNEL"\&VAL\$(C̄̄nnl) 
1334 GOSUB New_channl

1336 Print_chnnl:

1338 PRINT TABXY(3,2);Orange\$;

1340 CALL Auto_format(Chnnl*1.0)

1342 IF NOT (Wave_intrpt) THEN CALL Select_graphics(Which,Last1,Co_ords("))

1344

$1346 \quad 1$

1348

1350 !

1352 New_channl: !

1354

1356

1358

1360

1362

1364

1366

1368 RETURN

1370

1372

1374

1376

1378

1380

1382

1384

1386

1388

1390

1392

1394

1396 !

1398 Input_data_pnts: I

1400 OFF KEY

1402 OFF KBD

1404 OFF KNOB

1406 CLEAR SCREEN

1408 GCLEAR

1410 PRINT L_blue\$

1412 DIM Points\$(5)[20]

1414

1416

1418

1420

1422

1424 Frst:

1426

1428

1430

1432

1434

1436

1438

1440 Secnd:

Dp $\$=$ "Select Points

T\$ = "Available Points for the selected sweep speed."

IF (Time_per_div $>=1 . E-11$ ) AND (Time_per_div $<2 . E-11$ ) THEN Item_cnt $=2$

REDIM Points $\$(2)$

1442

1444

DATA " $100 "$

DATA " 400 "

RESTORE Frst

READ Points $\$(*)$

END IF

IF (Time_per_div $>=2.0 E-19$ ) AND (Time_per_div $<5 . E-19$ ) THEN Item_cnt $=3$

REDIM Points\$(3)

DATA "400"

DATA " 500 " 


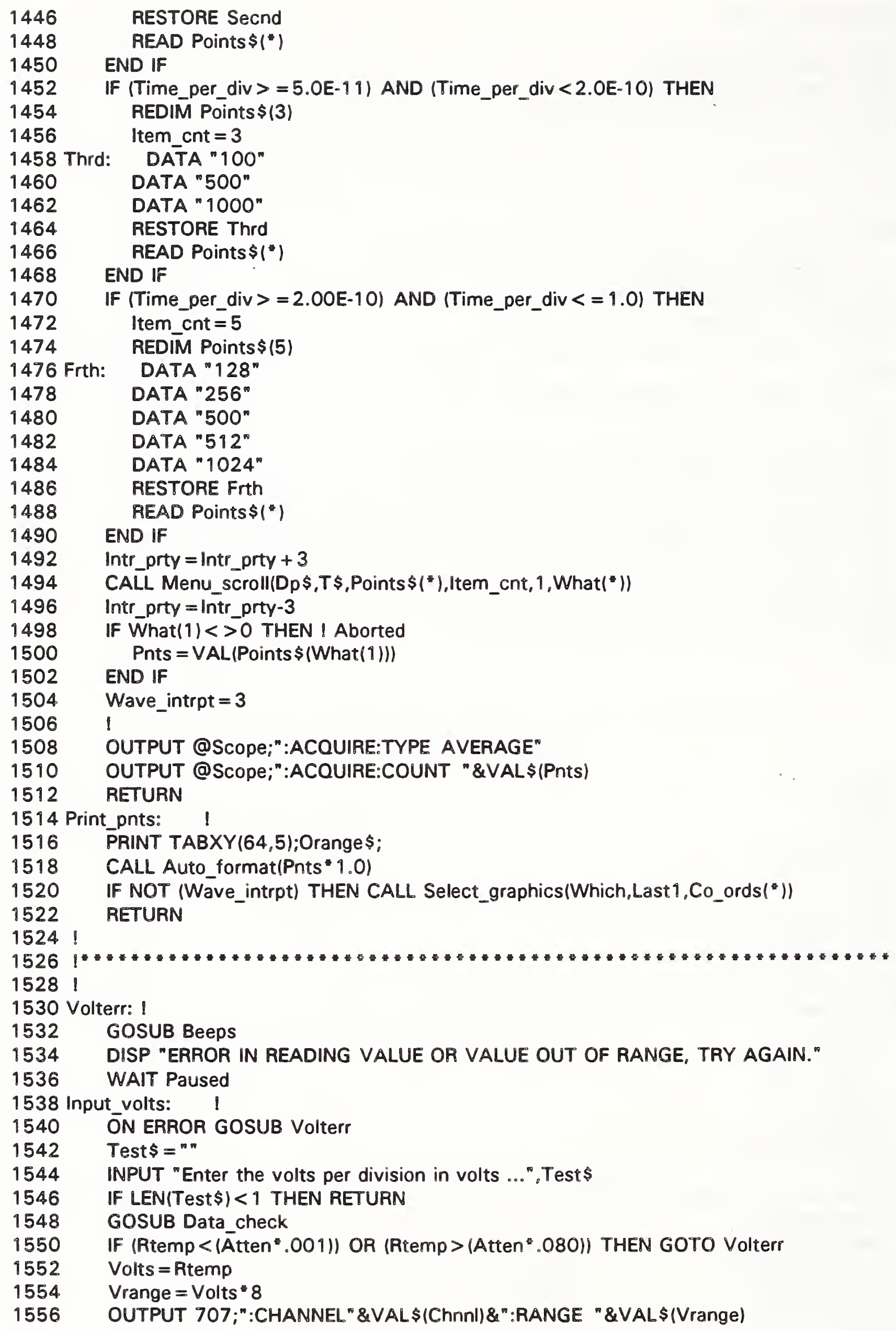




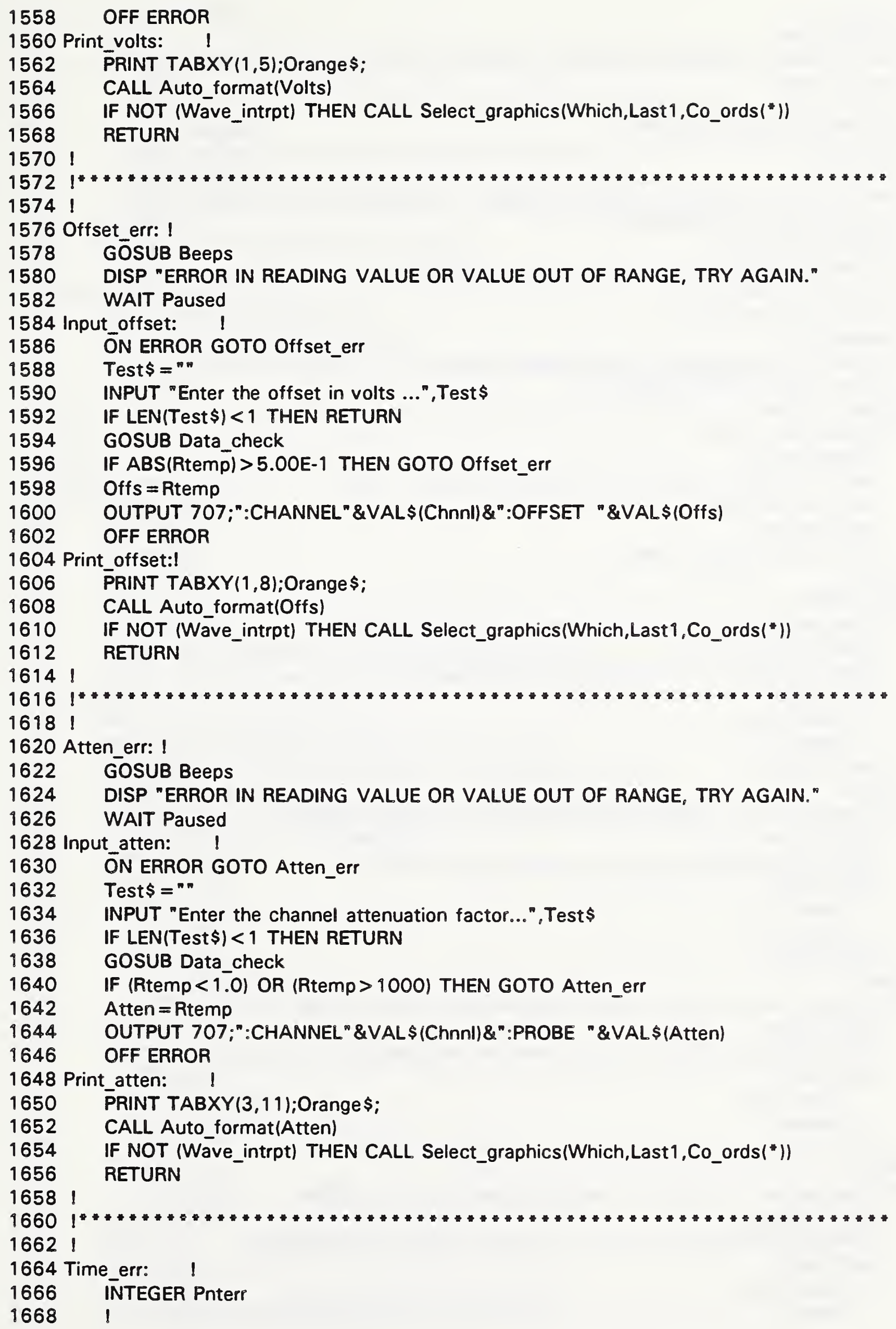


Pnterr $=0$

1678 Input_time:

\section{ON ERROR GOSUB Time_err}

Test $\$=$ क

Temp $=$ Pnts

INPUT "Enter the time per division in seconds ...",Test\$

IF LEN(Test\$) $<1$ THEN RETURN

GOSUB Data_check

IF (Rtemp < 1.OE-11) OR (Rtemp >1) THEN GOTO Time_err

Time_per_div $=$ Rtemp

Trange $=\overline{10} *$ Time_per_div

Rtemp $=$ Dly

OUTPUT 707;"TIMEBASE:RANGE " \&VAL\$(Trange)

OUTPUT 707;":TIMEBASE:DELAY7"

ENTER 707;Dly

IF Rtemp < > Dly THEN

GOSUB Beeps

DISP "The delay value is out of range. Delay set to "Dly,"." GOSUB Print_delay

WAIT Paused

DISP Orange\$;"DATA MENU *

END IF

OFF ERROR

IF (Time_per_div $>=1 . E-11$ ) AND (Time_per_div $<2 . E-11$ ) THEN

SELECT PntS

CASE 100,400 ! do nothing these are valid choices.

CASE ELSE

Pnts $=400$

Pnterr $=1$

END SELECT

END IF

IF (Time_per_div $>=2.0 E-11$ ) AND (Time_per_div $<5 . E-11$ ) THEN

SELEC̄T PntS

CASE 100,400,500! do nothing, these are valid selections.

CASE ELSE

Pnts $=500$

Pnterr $=1$

END SELECT

END IF

IF (Time_per_div $>=5.0 E-11$ ) AND (Time_per_div $<2.0 E-10$ ) THEN

SELEC̄T Ṕnts

CASE $100,500,1000$ ! do nothing, these are valid selections.

CASE ELSE

Pnts $=1000$

Pnterr $=1$

END SELECT

END IF

IF (Time_per_div $>=2.00 E-10$ ) AND (Time_per_div $<=1.0$ ) THEN SELECT PntS

CASE $128,256,500,512,1024$ ! do nothing, these are valid selections.

CASE ELSE

Pnts $=1024$

Pnterp $=1$ 
DISP "The number of points has been changed to the highest allowed value."

IDo nothing, accept scope value.

CASE " $B$ ", $b$ "

Pnts $=$ Temp

GOSUB Input_time

GOSUB Chk_d

CASE " $\mathrm{C}^{n},{ }^{\circ} \mathrm{C}^{\mathrm{N}}$

GOSUB Input_delay

CASE ELSE

CLEAR SCREEN

GOSUB Beeps

PRINT "That is not an option. Try again"

Choice $\$={ }^{n}$ "

GOTO Again

END SELECT

RETURN

1888 Read_delay_err: I

1890 ĠOSUB Beeps

1892 DISP "ERROR IN READING DELAY, TRY AGAIN." 


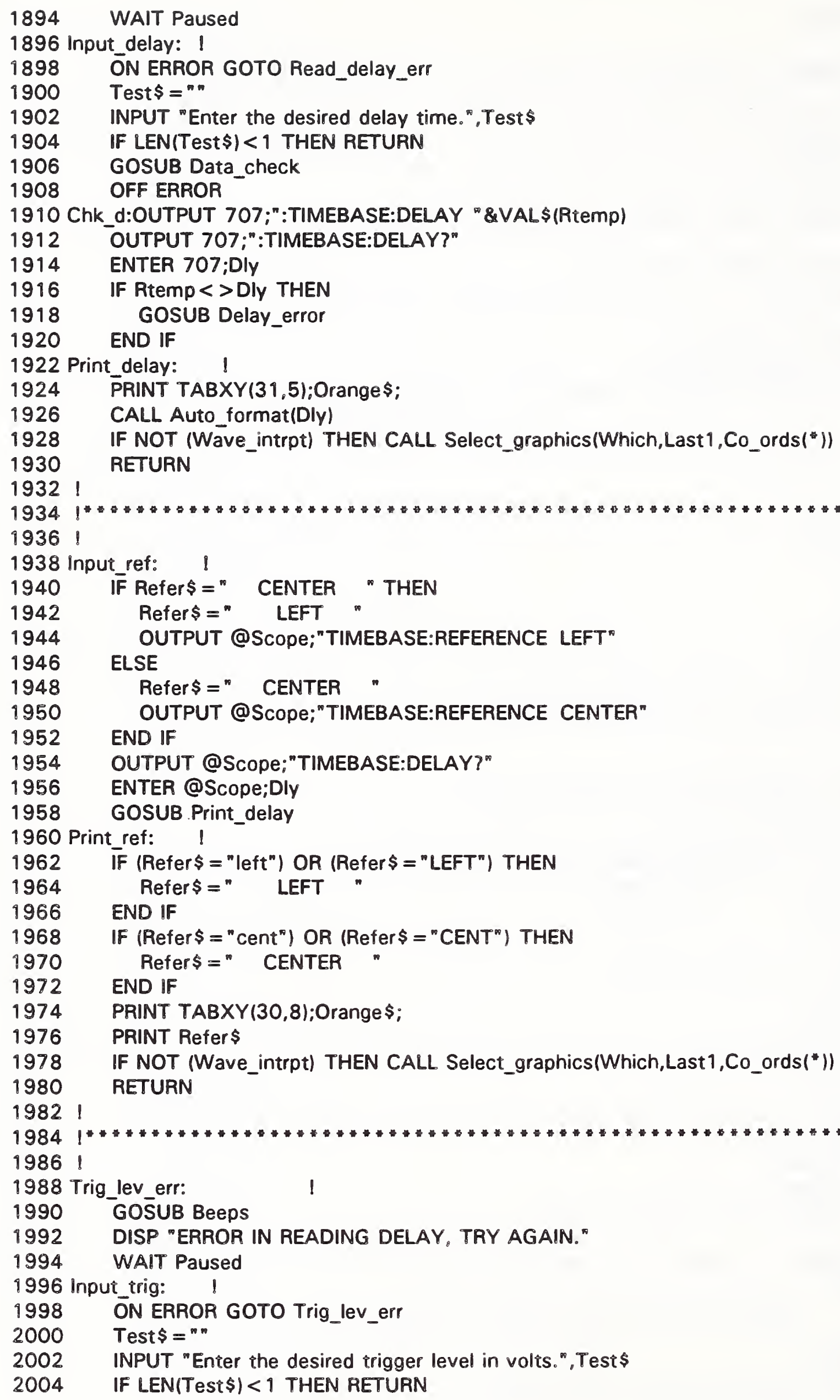


GOSUB Data_check

IF Rtemp $<-1$. OR Rtemp $>1$. THEN

GOSUB Beeps

2010

2012

2014

2016

2018

2020

2022

2024

2026 DISP Red \$;"VALUE OUT OF RANGE" ;Enh_off \$;"Try again."

WAIT 1.5

GOTO Input_trig

END IF

Trig = Rtemp

OUTPUT @Scope;":TRIGGER:LEVEL "\&VAL\$(Trig)

OFF ERROR

2030

PRINT TABXY $(60,8)$; Orange\$;

2032

2034

2036

2038

2040 !

2042 Manual_set: I

2044

2046

2048

2050

2052

2054

2056

2058

2060

2062

2064

2066

2068

2070

2072

2074

2076

2078

20801

2082 Chnl_err: 1

2084 BEEP

2086 DISP "Input out of range or disallowed value."

2088 WAIT Paused

2090 DISP "Try again ..."

2092 Get_chn:

2094 ON ERROR GOTO Chnnl_error

2096 Test $\$=$ "

2098 INPUT "Enter the acquisition channel number $(9-4) . "$, Test $\$$

2100 IF LEN(Test\$) $<1$ THEN RETURN

2102 Temp $=$ VAL(Test\$)

2104 OFF ERROR

2106

2108

2110

IF (Temp < 1) OR (Temp > 4) THEN GOTO Chnnl_error

Chnnl = Temp

2112 OUTPUT @Scope;"VIEW CHANNEL"\&VAL\$(Chnnl)

2114 OUTPUT @Scope;" :ACQUIRE:POINTS "\&VAL\$(Pnts)

2116 I The number of point can only be changed from the controller. 


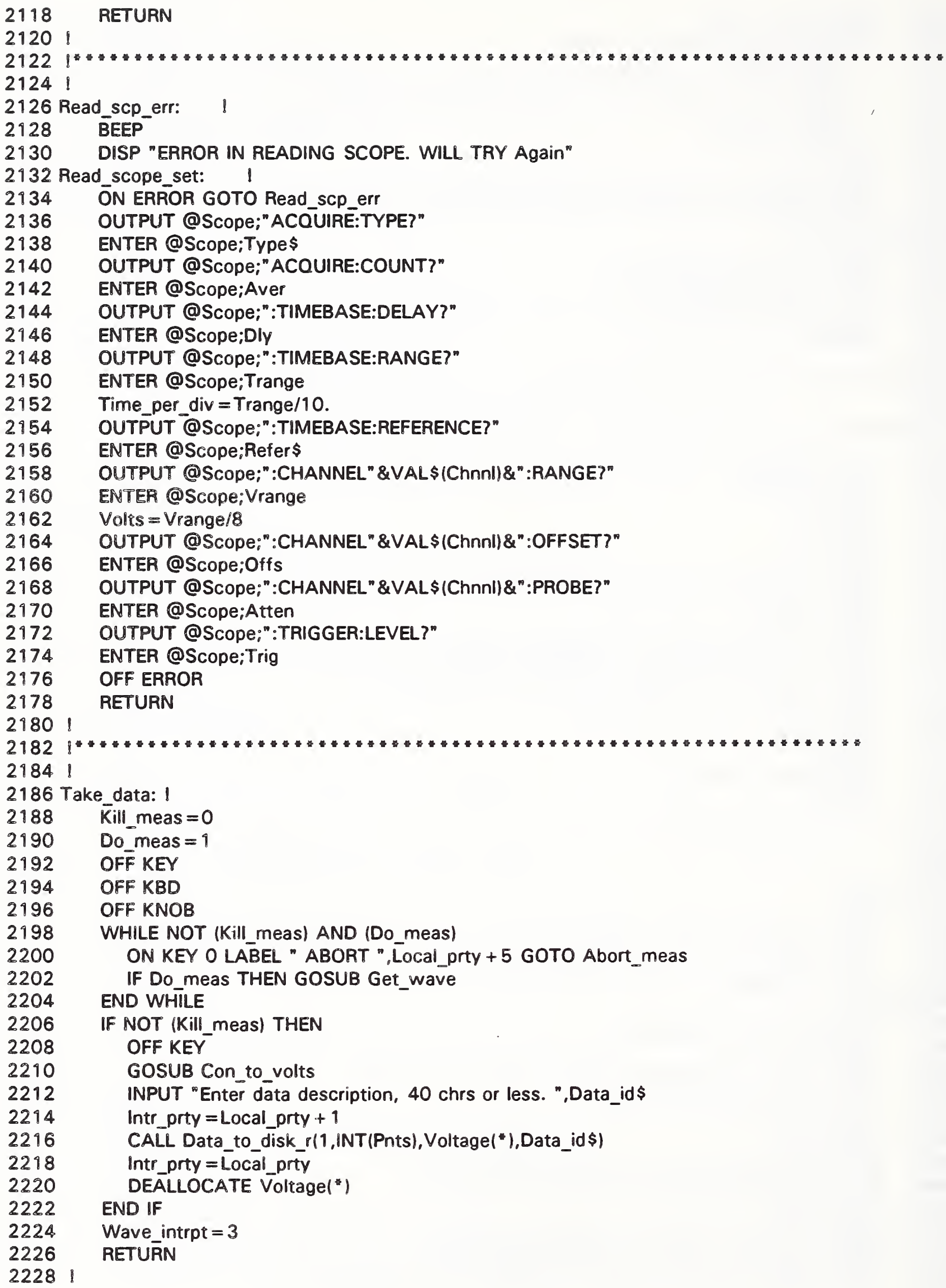


2234 Abort_meas: !

2236 CLEAR @Scope

2238 LOCAL @Scope

2240 Kill_meas $=1$

2242 CLEAR SCREEN

2244 GOSUB Beeps

2246 DISP Red\$;"MEASUREMENT ABORTED";Enh_off\$

2248

2250

2252

2254

2272 Data_error: !

2274 BEEP

2276 DISP "ERROR IN READING SCOPE. WILL TRY AGAIN"

2278 Get_wave:

2280 Do_meas $=0$

2282

2284

2286

2288 
OUTPUT @Scope;":ACQUIRE:POINTS "\&VAL\$(Pnts)

OUTPUT @Scope;" "CLS"

OUTPUT @Scope;" "SRE 32;"ESE 1"

OUTPUT @Scope;":DIGITIZE CHAN" \&VAL\$(Chnnl)\&";"OPC"

I The following loop allows for a keyboard abort key to be processed

I before completion of the DIGITIZE command.

WHILE NOT BIT(Stat,5) Stat=SPOLL(@Scope)

\section{END WHILE}

OUTPUT @SCOpe;":SYSTEM:HEADER OFF;:EOI ON"

OUTPUT @SCOpe;"WAVEFORM:SOURCE WMEMORY" \&VAL\$(Chnnl)\&"; FORMAT WORD"

OUTPUT @Scope;"WAVEFORM:DATA?"

ENTER @Scope USING "\#,A,D"; Header $\$$, Bytes

IF Bytes $=3$ THEN

END IF

ENTER @Scope USING "\#,3D";Length

IF Bytes $=4$ THEN

ENTER @Scope USING "\#,40";Length

\section{END IF}

Length $=$ Length $/ 2$

IF Pnts $<>$ Length THEN

DISP "The scope will not allow " ${ }^{\text {PPnts, }}$ " points.

WAIT Paused

DISP "The number of points is now " Length, "."

WAIT Paused

Pnts $=$ Length

\section{END IF}

REDIM Waveform(Length)

ENTER @Scope USING "\#,W";Waveform(")

ENTER @Scope USING "-K, $B^{n} ;$ End $\$$

OUTPUT @SCOpe:":WAVEFORM:YINCREMENT? ${ }^{*}$

ENTER @Scope;Yinc

OUTPUT @Scope; ${ }^{\text {"}}$ WAVEFORM:YORIGIN?"

ENTER @Scope;Yorg

OUTPUT @Scope ${ }^{\prime \prime}:$ WAVEFORM:YREFERENCE?"

ENTER @Scope;Yref

OUTPUT @Scope;":WAVEFORM:XINCREMENT?"

ENTER @Scope;Xinc

OUTPUT @Scope;":WAVEFORM:XORIGIN?"

ENTER @Scope;Xorg

OUTPUT @Scope; "WAVEFORM:XREFERENCE?"

ENTER @Scope;Tref

OFF ERROR

IF Bug 1 THEN

FOR I $=9$ TO Pnts

PRINT Waveform(I) NEXT I

END IF

END IF

RETURN 


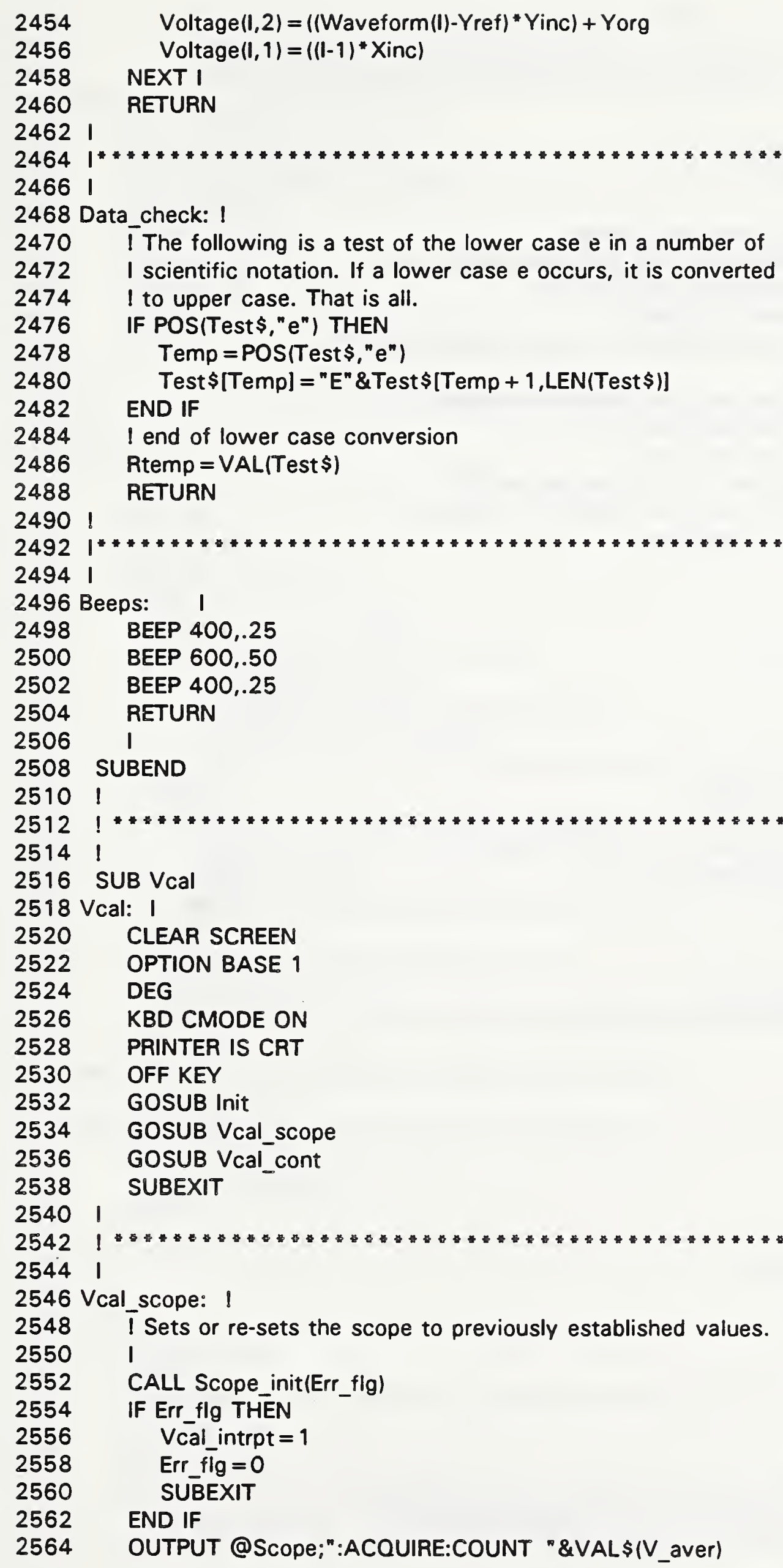


2574 Init:।

COM /interrupts/ INTEGER intr_prty

COM /Sys_msi/ Msi_id\$[20]

2580

2582

COM /Sys/ Sys_id\$[10]

COM /Bugs/ INTTEGER Bug1,Bug2,Bug3,Printer

2584

2586

2588

2590

2592

2594

2596

2598

2600

2602

2604

2606

2608

2610

2612

2614

2616

2618

2620

2622

2624

2626

2628

2630

2632

2634

2636

2638

2640

2642

2644

2646

2648

2650

2652

2654

2656

2658

2660

2662

2664 I

2666 2668 ।

2670 Graphs_vc1300:?

2672

COM /Files/ Diskdrive\$[20],Filename\$[14],Ms_path\$[500]

COM /Hue/ Rev_vid\$[1],Enh_off $\$[1]$, Underline $\$[1]$

COM /Hue/ Red $\$$ [1], Orange $\$$ [1], L_blues[1]

COM /Mnu/ INTEGER Interrupted, Which, T_which,V_which,Allowd\$(")

COM Mnu/ INTEGER Stp

COM /Scope/ REAL Time_per_div, Volts, Trange, Vrange,Dly

COM /Scope/ REAL Probe_fac, Offs, Trig,Atten

COM /Scope/ INTEGER Aver,Pnts, Chnnt

COM /Scope/ Type\$[30],Refer\$[14],@Scope,Mode\$[30]

COM /Ncal_vals/ INTEGER V_aver, Interval,REAL_V_step,V_min

COM /Slect// INTEGER Last1, Tiast, Vlast

$!$

1

DIM Version\$[80]

DIM Test\$[160],Data_id\$[40]

DIM Choice \$ [15]

!

!

INTEGER Co_ords $(4,4)$,Lwrlftx,Lwrlfty,Upperrtx,Upperntty

INTEGER Num_of_choices,What (1)

INTEGER I,J,Pen, Knobcount, Counter

INTEGER Error_flag,Beep_flag,Local_prty,Valid,Datacount

INTEGER Filesize,Baddatä,Endpoint, Print_val

INTEGER Yref,Temp,Err_flg

1

1

REAL Data_entered,Data_set_count

REAL Yinc, Yor, Rtemp,Add,Waveform(32767)

Local_prty $=$ intr_prty

Ftype $\$=$ "BDAT"

Filesize $=500$

Last $1=0$

PEN 1

Beep_flag $=0$

Paused $=1.5$

I

IVoltage calibration variables

!

1

RETURN

2676

IThis routine fills the array used by the select_graphics

Iroutine. The array contains the coordinates for the

Irectangles drawn in select_graphics. 

lused to make all rows of equal length.

2684 Frst: DATA $30,900,176,7711,1$

2686 DATA $665,900,245,7711,2$

2688 DATA $30,675,176,7712,1$

2690 DATA $665,675,245,77 ! 2,2$

2692 RESTORE Frst

2718 intr_prty = Local_prty + 1

2720 LOOP

IF Vcal_intppt $=1$ THEN

IF Sys_id $\$[1,4]={ }^{\text {"S }} 300$ " THEN CONTROL CRT,5; 1 SEPARATE ALPHA FROM GRAPHICS END IF GOSUB Graphs_vcl300

\section{END IF}

IF (Vcal_intrpt $=1)$ OR (Vcal_intrpt $=3$ ) THEN GOSÜB Backgrnd GOSUB Fill_vals

\section{END IF}

CALL Select_graphics(V_which, Vlast,Co_ords (*))

IF Vcal_intrpt THEN GOSUB Vcal_menu Interrupted $=1$

ON KEY 9 LABEL "EXIT PROGRAM",Local_prty + 3 GOTO Ret 1

2754 Ret1: Stp $=1$ IThis will cause the entire program to end.

2756 Interrupted $=0$

2758 Ret2: OFF KEY IThis will exit only this subprogram.

2760

2762

2764

2766

2768

2770 !

2772 Vcal_menu: $\quad$ I

2776

2778

2788

$$
\text { OFF KEY }
$$

OFF KBD

OFF KNOB

Knobcount $=0$

DISP Oranges;"VCAL MENU "

ON KBD,Local_prty +1 GOSUB Process_kbd

ON KNOB.01,Local_prty + 1 GOSUB Move_pointer 
ON KEY 1 LABEL "ACQUIRE DATA "LLcal_prty +1 GOSUB Vcal_acq

Test $\$=$ KBD $\$$

IF LEN(Test\$) $=1$ AND Test $\$[1,1]<>$ CHR $\$(32)$ THEN RETURN

Vcal_intrpt $=2$

IF Test\$ $[1,1]=$ CHR\$(32) THEN REPEAT

IF $V$ _which < Num_of_choices THEN

ELSE $V_{-}$which $=V_{-}$which +1

$$
V \text { which }=1
$$

END IF

UNTIL Co_ords(V_which, 1$)<>0$

CALL Select_graphics(V_which, Vlast,Co_ords $(")$ )

\section{END IF}

IF Test $\$(1,1]<>$ CHR\$(255) THEN RETURN

SELECT Test $\$[2,2]$

CASE CHR\$(255)

1 do nothing, this is a CTRL character

CASE " $V$ " "T" Idown arrow

REPEAT

IF $V$ _which $<=$ (Num_of_choices-Rowsize) THEN

$V_{\text {_which }}=V_{\text {_ which }}+$ Rowsize

ELSE

$V_{\text {_ which }}=$ Rowsize-(Num_of_choices $-V_{\text {_ }}$ which)

END IF

UNTIL Co_ords(V_which, 1$)<>0$

CASE "A⿱ " "W" lup arrow

REPEAT 
2956

2958 2960 !

2962 Move_pointer: !

2964

2966

2968

2970

2972

2974

2976

2978

2980

2982

2984

2986

2988

2990

2992 2994 !

2996

2998 !

3000 Backgrnd: I

3002

3004

3006

3008

3010

3012

ELSE

END IF

REPEAT

ELSE

END IF

REPEAT

ELSE'

END IF

CASE ELSE

END SELECT

RETURN

Vcal intrpt $=2$

REPEAT

ELSE

END IF

Knobcount $=0$

RETURN

!

CLEAR SCREEN

END IF
IF $V$ which $>$ Rowsize THEN $V_{-}$which $=V_{-}$which-Rowsize

$V_{-}$which $=V_{\text {_ }}$ which + (Num_of_choices-Rowsize)

UNTIL Co_ords(V_which, 1$)<>0$

CASE " $<n, " \bar{H}^{n},{ }^{\prime \prime n}-$ lleft arrow, prev key

IF $V$ which $>1$ THEN

$V_{-}$which $=V_{-}$which 1

$V$ _which $=$ Num_of_choices

UNTIL Co ords (V_which, 1$)<>0$

CASE $">n, " \bar{G} ", n, "$ lright arrow, next key

IF $V$ which < Num_of_choices THEN

$$
V_{-} \text {which }=V_{\text {_ }} \text { which }+1
$$

$V_{-}$which $=1$

UNTIL Co_ords(V_which, 1$)<>0$

BEEP $80, . .1$

CALL Select_graphics(V_which,Vlast,Co_ords $\left.\left({ }^{*}\right)\right)$

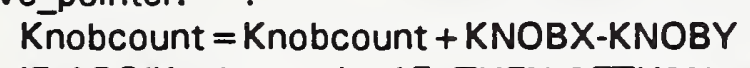

Knobcount $=$ Knobcount + KNOBX-KNOBY

IF ABS(Knobcount) $<15$ THEN RETURN

IF Knobcount $>0$ THEN

$V_{\text {_ }}$ which $=V_{\text {_ }}$ which +1

$V_{\text {_which }}=V_{\text {_which }}$ 1

IF $V_{-}$which $<1$ THEN $V_{-}$which $=$Num_of_choices

IF $V_{\text {_ }}$ which $>$ Num_of_choices THEN $\bar{V}_{-}$which $=1$

UNTIL Co_ords(V_which, 1$)<>0$

CALL Select_graphics (V_which, Vlast,Co_ords (*)|

IF SYS id $\$ 1,4]={ }^{\text {" }}$ S300" THEN

MERGG ALPHA WITH GRAPHICSIThis gives back the colors to the alpha !plane. 
PRINT TABXY $(1,1)$;Rev_vid\$;L_blue\$;" \# OF VOLTAGE ";Enh_off\$;

PRINT TABXY(1,7);Rev_vid\$;L_blue\$;" VOLTAGE STEP ";Enh_off\$;

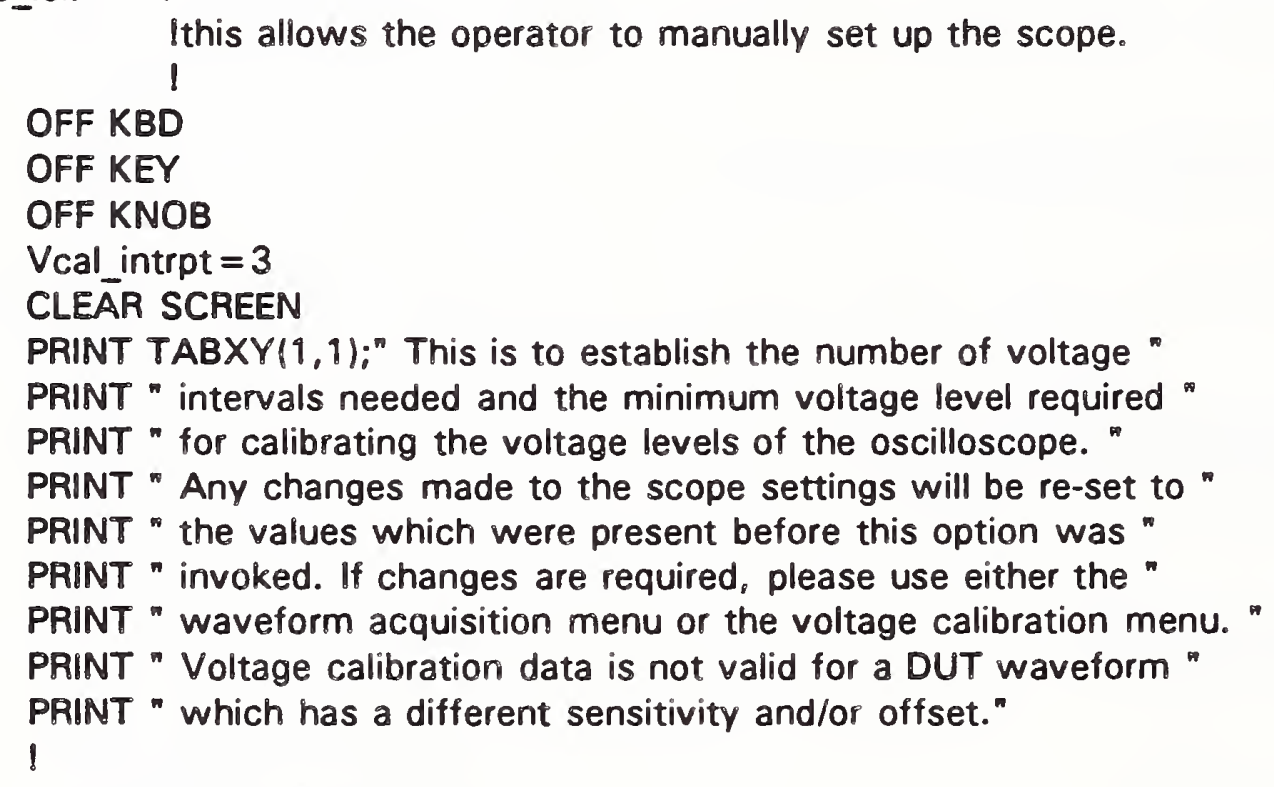
Ithis allows the operator to manually set up the scope. 1

OFF KBD

OFF KEY

OFF KNOB

Vcal_intrpt $=3$

CLEAR SCREEN

PRINT TABXY $(1,1) ;$ " This is to establish the number of voltage "

PRINT " intervals needed and the minimum voltage level required "

PRINT " for calibrating the voltage levels of the oscilloscope."

PRINT "Any changes made to the scope settings will be re-set to "

PRINT "the values which were present before this option was"

PRINT " invoked. If changes are required, please use either the "

PRINT " waveform acquisition menu or the voltage calibration menu."

PRINT "Voltage calibration data is not valid for a DUT waveform "

PRINT " which has a different sensitivity and/or offset." I 
DISP "Input out of range or disallowed value."

WAIT Paused

DISP "Try again ..."

Get_chn:

ON ERROR GOTO Chnl_err

Test $\$=" n$

INPUT "Enter the acquisition channel number $(1-4) .{ }^{\text {" , Test } \$}$

IF LEN(Test\$) $<1$ THEN RETURN

Temp $=$ VAL (Test $\$$ )

OFF ERROR

IF (Temp<1) OR (Temp >4) THEN GOTO Chnnl_error OUTPUT @Scope;"BLANK CHANNEL" \&VAL\$(Chnnnl)

Chnnl $=$ Temp

OUTPUT @Scope;"VIEW CHANNEL"\&VAL\$(Chnnl)

1 The number of point can only be changed from the controller.

RETURN $1 *$

3182 Choose average:

OFF KEY

OFF KNOB

OFF KBD

CLEAR SCREEN

GCLEAR

PRINT L_blue\$

DIM Dpsi $[80]$

DIM T\$[52]

Dp\$ = "Select Average "

$T \$=$ "Available Averages (powers of 2 ) "

Intr_prty $=$ Intr_prty +3

CALL Menu_scroll(Dp\$,T\$,Allowd $\$(*), 92,9$, What (*)) Intr_prty $=$ Intr_prty-3

If What $(1)<>0$ THEN! Aborted

V_aver = VAL(Allowd $\$($ What $(1))$ )

END IF

Vcal_intrpt $=3$

!

OUTPUT @Scope;":ACQUIRE:TYPE AVERAGE"

OUTPUT @Scope;":ACOUIRE:COUNT "\&VAL\$(V_aver)

3226 Print

\section{ave: I}

PRINT TABXY $(60,4)$;Orange\$;

CALL Auto_format(V_aver*1.0)

RETURN I 
3242 Interval error: !

3244 GOS̄UB Beeps

3246 DISP "ERROR IN READING VALUE OR DISALLOWED VALUE, TRY AGAIN."

3248 WAIT Paused

3250 Input interval: 1

3252 ŌN ERROR GOTO Interval_error

3254 Test $\$=$ "

3256 INPUT "Enter the number of voltage intervals." ,Test\$

3258 IF LEN(Test\$) $<1$ THEN RETURN

3260 Temp $=$ VAL (Test $\$$ )

3262 OFF ERROR

3264 If Temp < 1 THEN GOTO Interval_error

$3266 \quad$ Interval $=$ Temp

3268 Print intervls: $\quad$ I

3270 PRINT TABXY(2,4); Orange\$:

3272 CALL Auto format (Interval \%.0)

3274 IF NOT (Vcal_intrpt) THEN CALL Select_graphics(V_which,Vlast,Co_ords("))

3276 RETURN

3278 !

3280

3282 !

3284 Step error: !

3286 GOSUB Beeps

3288

3290

DISP "ERROR IN READING VALUE OR VALUE OUT OF RANGE. TRY AGAIN."

3292 Input step:

3294 ON ERROR GOTO Step_error

3296 Test $\$=$ *

3298

3300

3302

3304

3306

3308

3310

3312 Print_step:

DISP "Enter the voltage step size."

INPUT "This must be a positive, real number." Test\$

IF LEN(Test $\$$ ) $<1$ THEN RETURN

GOSUB Data_check

OFF ERROR

IF Rtemp<0. THEN GOTO Step_error

$V$ step $=$ Rtemp

3314

3316

3318

3320

3322

3324

3326 !

3328 Minvolt_error: !

3330 GOSUB Beeps

3332

3334

3336 Input_min_volt:

3338

3340

3342

3344

3346

3348

PRINT TABXY(1,10);Orange\$:

CALL Auto_format(V_step)

IF NOT (Vcal_intrpt) THEN CALL Select_graphics(V_which,Vlast,Co_ords (")) RETURN

DISP "ERROR IN READING VALUE OR VALUE OUT OF RANGE. TRY AGAIN."

WAIT Paused

ON ERROR GOTO Minvolt_error

Test\$ $=$ " "

INPUT "Enter the minimum calibration voltage."

IF LEN(Test\$) $<1$ THEN RETURN

GOSUB Data_check

OFF ERROR 


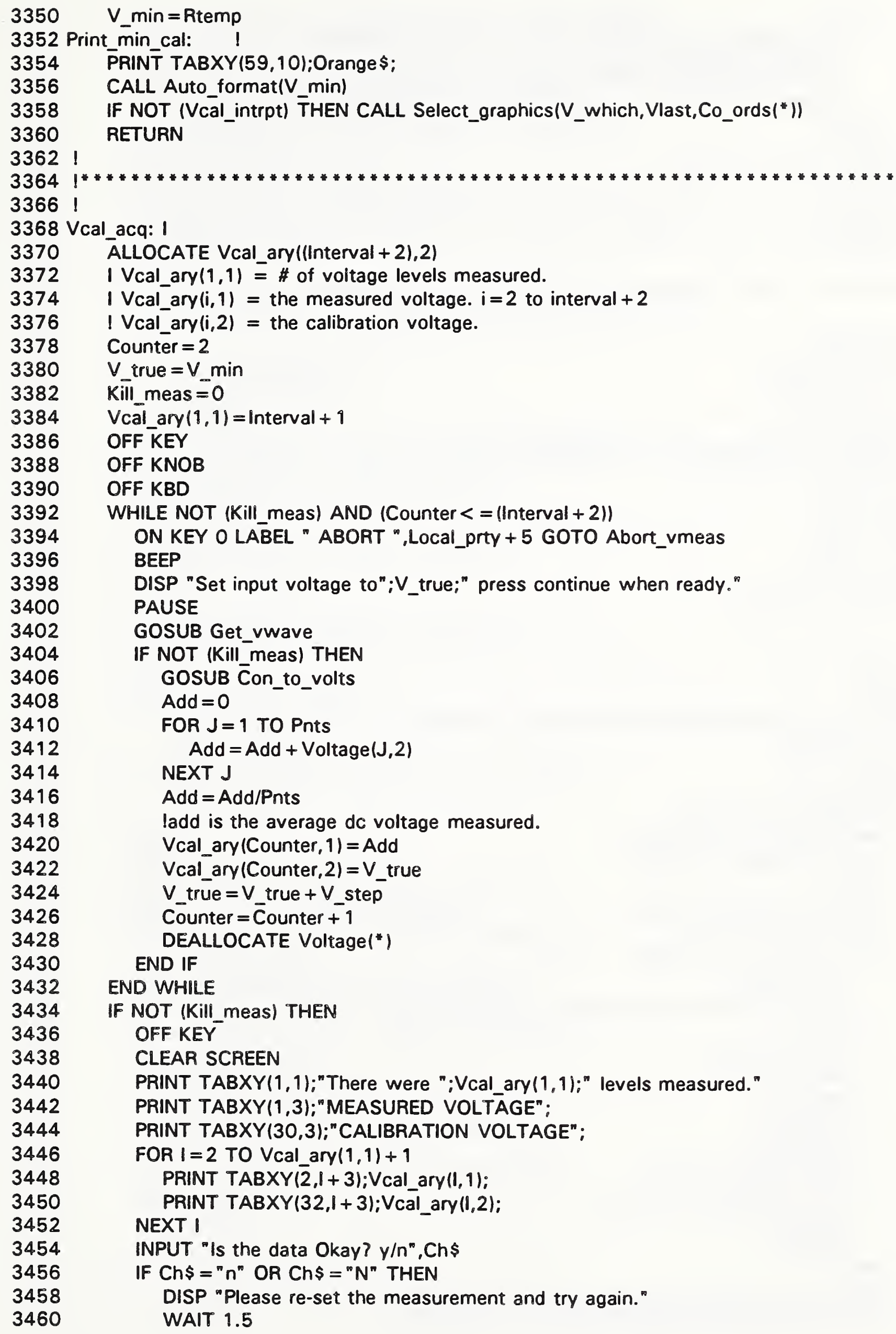


3490 Abort_vmeas: !

3492

3494

3496

3498

3500

3502

3504

3506

3508

3510

3512

$3514 !$

3516 !

3518 Dat

3520

3522

3524

3526

3528

3530

3532

3534

3536

3538

3540

3542

3544

3546

3548

3550

3552

3554

3556

3558

3560

3562

3564

3566

3568

3570

3572

\section{ELSE}

INPUT "Enter data description, 40 chrs or less. "Data_id\$

Intr_prty = Local_prty +1

CALL Data_to_disk_r(1,INT(/Counter + 1)),Vcal_ary/"),Data_id \$)

END IF

$$
\text { Intr_prty = Local_prty }
$$

END IF

DEALLOCATE Vcal_ary(")

OFF KEY

Vcal_intrpt $=3$

RETURN 
OUTPUT @Scope;":TIMEBASE:RANGE "\&VAL\$(Trange)

OUTPUT @Scope;":CHANNEL" \&VAL\$(Chnnl)\&":OFFSET "\&VAL\$(Offs)

OUTPUT @Scope;":CHANNEL" \&VAL\$(Chnnl)\&n:RANGE "\&VAL\$(Vrange)

OUTPUT @Scope;":ACQUIRE:TYPE AVERAGE"

OUTPUT @Scope;":ACQUIRE:COUNT "\&VAL\$(V_aver)

OUTPUT @Scope;":ACQUIRE:POINTS "\&VAL\$(Pnts)

OUTPUT @Scope;" * CLS"

OUTPUT @Scope;" *SRE 32;"ESE 1"

OUTPUT @Scope;":DIGITIZE CHAN"\&VAL\$(Chnnl)\&"; OPC"

I Digitizes the channel and asks for the operation complete

I status indicator flag.

1

1 This loop enables the keyboard interrupt key called ABORT.

I The abort key will work even when the scope is busy.

!

WHILE NOT BIT(Stat,5)

Stat=SPOLL(@Scope)

END WHILE

OUTPUT @Scope;":SYSTEM:HEADER OFF;:EOI ON"

OUTPUT @SCOPe;"WAVEFORM:SOURCE WMEMORY"\&VAL\$(Chnnl)\&"; FORMAT WORD" OUTPUT @Scope;"WAVEFORM:DATA?"

ENTER @Scope USING "\#,A,D"; Header\$,Bytes

IF Bytes $=3$ THEN

ENTER @Scope USING "\#,3D";Length

END IF

IF Bytes $=4$ THEN

END IF

ENTER @Scope USING "\#,4D";Length

Length $=$ Length $/ 2$

IF Pnts $<>$ Length THEN

DISP "The scope will not allow ",Pnts, "points."

WAIT Paused

DISP "The number of points is now ",Length," ".

WAIT Paused

Pnts = Length

END IF

REDIM Waveform(Length)

ENTER @Scope USING "\#,W";Waveform( $\left.{ }^{*}\right)$

ENTER @Scope USING "-K, $B^{n} ;$ End\$

OUTPUT @SCOpe;":WAVEFORM:YINCREMENT?"

ENTER @Scope;Yinc

OUTPUT @SCOpe;" :WAVEFORM:YORIGIN?"

ENTER @Scope;Yorg

OUTPUT @SCOpe;" :WAVEFORM:YREFERENCE?"

ENTER @Scope;Yref

OUTPUT @SCOpe;":WAVEFORM:XINCREMENT?"

ENTER @Scope;Xinc

OUTPUT @ScOpe;":WAVEFORM:XORIGIN?"

ENTER @Scope;Xorg

OUTPUT @Scope;":WAVEFORM:XREFERENCE?"

ENTER @Scope;Tref

OFF ERROR

IF Bug1 THEN

FOR $I=1$ TO Pnts PRINT Waveform(I) NEXT I 


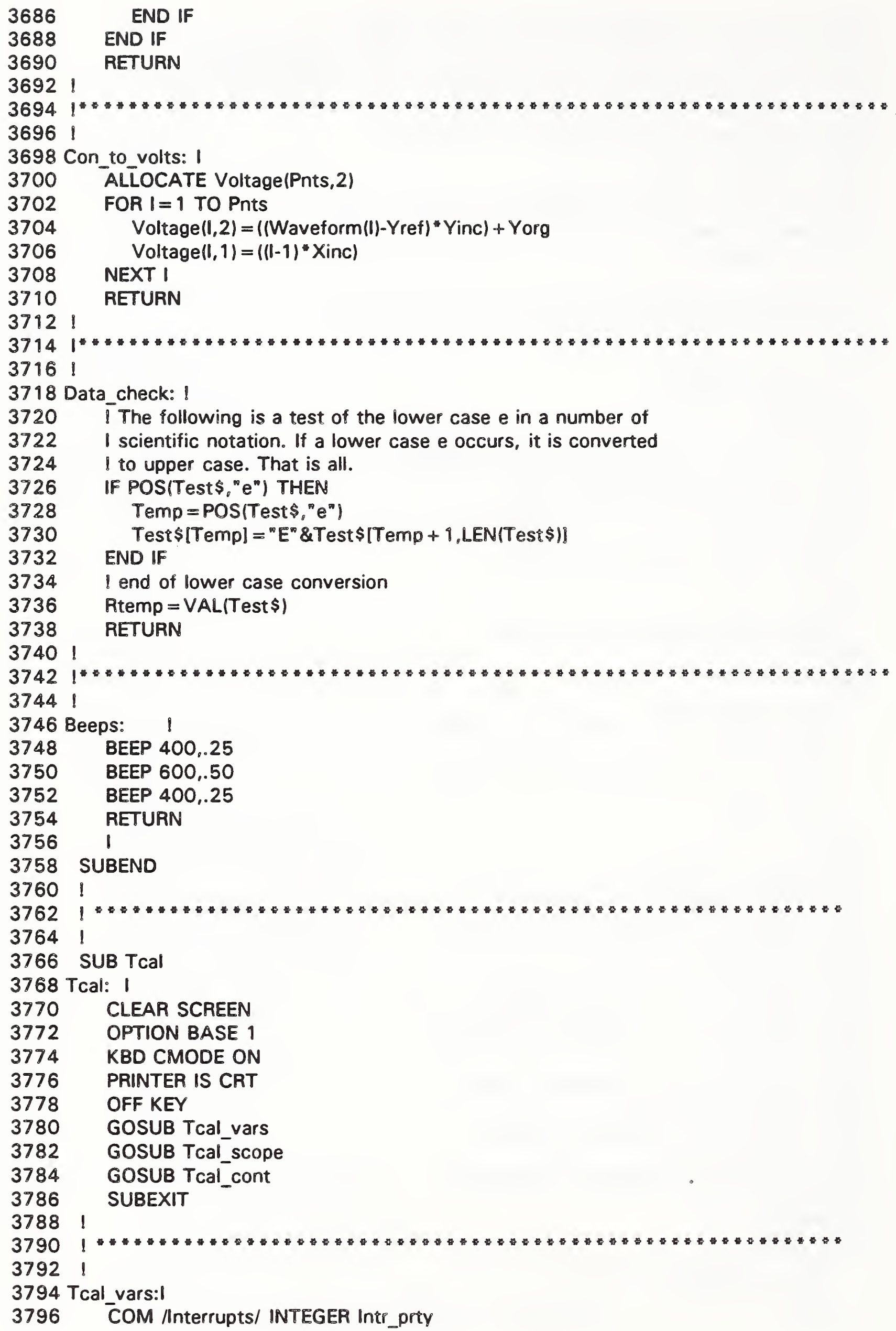


COM /Sys_msi/ Msi_id\$[20]

COM /Sys/ Sys_id $\$[10]$

COM /Bugs/ INTTEGER Bug1,Bug2,Bug3,Printer

COM /Files/ Diskdrive $\$[20]$,Filename $\$[14]$,Ms_path $\$[500]$

COM /Hue/ Rev_vid\$[1],Enh_off\$[1],Underline\$[1]

COM /Hue/ Red $\$$ [1], Oranges[1],L_blue\$[1]

COM /Mnu/ INTEGER Interrupted, Which,T_which,V_which,Allowd $\$$ (")

COM /Mnu/ INTEGER Stp

COM /Scope/ REAL Time_per_div, Volts, Trange,Vrange,Dly

COM /Scope/ REAL Probe_fac,Offs, Trig, Atten

COM /Scope/ INTEGER Aver,Pnts,Chnnl

COM /Scope/ Type\$[30],Refer\$[14],@Scope,Mode\$[30]

COM $\pi$ cal_vals/ INTEGER Zero_x,Ls_prs,Slope,T_aver,REAL Freq

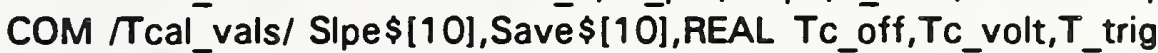

COM /Line_fit/ INTEGER Wav(1024)

COM /Slect/ INTEGER Last1,Tlast, Vlast

3828

3830

3832

3834

3836

3838

3840

3842

3844

3846

3848

3850

3852

3854

3856

3858

3860

3862

3864

3866

3868

3870

3872

3874

3876

3878

3880

3882

3884

3886

3888

3890

3892

3894

3896 !

3898

39001

3902 Tcal_scope: 1

3904

3906

3908

!

DIM Version $\$[80]$

DIM Test\$[160],Data_id\$[40]

DIM Choice\$[15]

1

INTEGER Co_ords $(9,4)$, Lwrlftx,Lwrlfty,Upperrtx,Upperrtty

INTEGER Num_of_choices, What (1)

INTEGER I,Cntr,Pen,Knobcount

INTEGER Error_flag,Beep_flag,Local_prty,Valid,Datacount

INTEGER Filesize,Baddata,Endpoint,Print_val

INTEGER Yref,Temp,Tcal_intrpt,Kount

INTEGER Strt,End_pnt,Midval,Err_flg

I

1

REAL Data_entered,Data_set_count

REAL Yinc,YYor,Rtemp,Zero_level,Ln_slp,Intrcpt

REAL Maxval,Minval, Waveform(32767)

1

Local_prty = Intr_prty

Ftype $\$=$ "BDAT"

Filesize $=32767$

Last $1=0$

PEN 1

Beep_flag $=0$

Paused $=1.5$

1

!Time calibration variables

I

I

RETURN

CALL Scope_init(Err_flg)

IF Err_flg THEN

Tcal_intrpt $=3$ 
3910

3912

3914

3916

3918

3920

3922

3924

3926

3928

39301

3932

$3934 !$

3936 !

3938 Graphs_ary300:!

3940

3942

3944

3946

3948

3950

3952 Firs

3954

3956

3958

3960

3962

3964

3966

3968

3970

3972

3974

3976

3978

3980

3982

3984 !

3986 !

3988 Tcal_cont: I

3990

3992

3994

3996

3998

4000

4002

4004

4006

4008

4010

4012

4014

4016

4018

4020

Err_flg $=0$

SUBEXXIT

END IF

RETURN

$$
\text { ! }
$$

$1 * *$

RESTORE First

Rowsize $=3$

RETURN

I

(1)

1

OFF KEY

Tcal_intrpt $=1$

LOOP

END IF

END IF

END IF

OUTPUT @Scope:":CHANNEL"\&VAL\$(Chnnl)\&":OFFSET "\&VAL\$(TC_off)

TC_vrange $=$ TC_volt $" 8$

OUTPUT @Scope; ;":CHANNEL" \&VAL\$(Chnnl)\&" :RANGE "\&VAL\$(TC_vrange)

OUTPUT @Scope;":ACQUIRE:COUNT "\&VAL\$(T_aver)

OUTPUT @Scope;"TRIGGER:LEVEL_"\&VAL\$(T_trig)

IThis routine fills the array used by the select_graphics

lroutine. The array contains the coordinates for the

Irectangles drawn in select_graphics.

lif the data is all zeros, this is a null field and is

lused to make all rows of equal length.

IThese coordinates are for the system 300 machines.

irst:DATA 30,943,176,34 11,1 irequency

DATA $372,943,245,3411,2$ save the waveform

DATA $714,943,220,34 ! 1,3$ averages

DATA $30,830,176,3512,1$ volts

DATA $372,830,245,35 ! 2,2$ calibrate on (pos/neg slope)

DATA $714,793,220,7012,3$ number of points for the moving average

DATA $30,717,176,35 ! 3,1$ offset $\mathrm{mv}$

DATA $372,680,245,7013,2$ number of least squares point pairs

DATA $714,680,220,3513,3$ trigger level for time calibration

READ Co_ords(")

Num_of_choices $=9 \quad \mid$ total number of on screen choices

IF Tcal_intrpt $=1$ THEN

IF Sys id $\$[1,4]=$ "S300" THEN

CONTTROL CRT, $5 ; 1$

SEPARATE ALPHA FROM GRAPHICS

GOSUB Graphs_ary 300

IF (Tcal_intrpt $=1)$ OR (Tcal_intrpt $=3)$ THEN

GOSUB Background

GOSUB Fill_in

CALL Select_graphics(T_which,Tlast,Co_ords( $\left.{ }^{*}\right)$ ) 

Interrupted $=1$

4030 Ret $1:$ Stp $=1$ IThis will cause the entire program to end.

4032 Ret2: OFF KEY IThis will exit only this subprogram.

4034

4036

40381

CLEAR SCREEN

4040

40421

4044 Tcal_menu: $\quad 1$

4046 Tcal_intrpt $=0$

4048 OFF KEY

4050 OFF KBD

4052 OFF KNOB

4054 Knobcount $=0$

4056

4058

4060

4062

4064

4066

4068

4070

4072

4074

4076

4078

4080

4082

4084

4086

4088

4090

4092

4094

4096

4098

4100

4102

4104

4106

4108

4110

4112

4114

4116

4198

4120

4122

4124

4126

4128

4130

4132

ON KBD, Local prty +1 GOSUB Process kbd

ON KNOB .01,Loca!_prty + 1 GOSUB Move_pointer

ON KEY 1 LABEL "ACCOUIRE DATA ",Local_prty + 1 GOSUB Tcal_data

ON KEY 5 LABEL "MAIN MENU ",Local_prty + 1 GOTO Ret2

ON KEY 7 LABEL "MANUAL SETUP",Local_prty + 1 GOSUB Tcal_set

SELECT T_which

1

CASE 1 Ifrequency

ON KEY 3 LABEL "CHANGE VALUE",Local_prty+ 1 GOSUB Input_freq

CASE 2 Isave the sinewave?

ON KEY 3 LABEL "CHANGE VALUE",Local_prty + 1 GOSUB Save_wave 1

CASE 3 I \# of point pairs

ON KEY 3 LABEL "CHANGE VALUE",Local_prty + 1 GOSUB Choose_average 1

CASE 4 I voltage

ON KEY 3 LABEL "CHANGE VALUE",Local_prty + 1 GOSUB Input_volts I

CASE 5 I slope

ON KEY 3 LABEL "CHANGE VALUE",Local_prty + 1 GOSUB Which slope !

CASE 6 ! points for moving average

ON KEY 3 LABEL "CHANGE VALUE",Local_prty + 1 GOSUB Moving_points !

CASE 7 I offset

ON KEY 3 LABEL "CHANGE VALUE",Local_prty + 9 GOSUB Input_offset !

CASE 8 I least squares point pairs

ON KEY 3 LABEL "CHANGE VALUE",Local_prty +1 GOSUB Point_pairs 1

CASE 9 I trigger level

ON KEY 3.LABEL "CHANGE VALUE",Local_prty +1 GOSUB Trigger_|v|

1

CASE ELSE

GOSUB Beeps

DISP "ERROR PARAMETER OUT OF RANGE"

WAIT Paused

END SELECT 


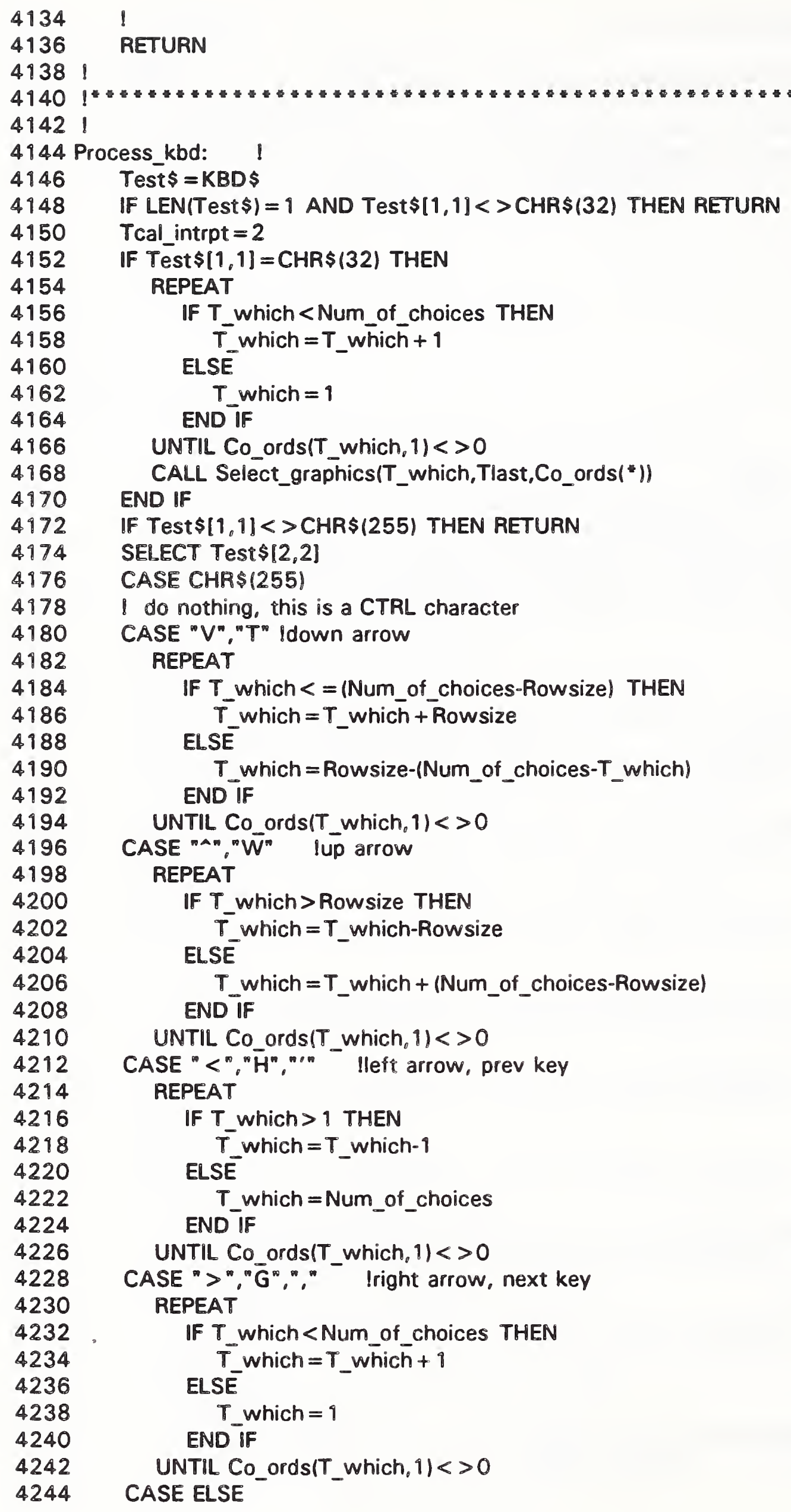




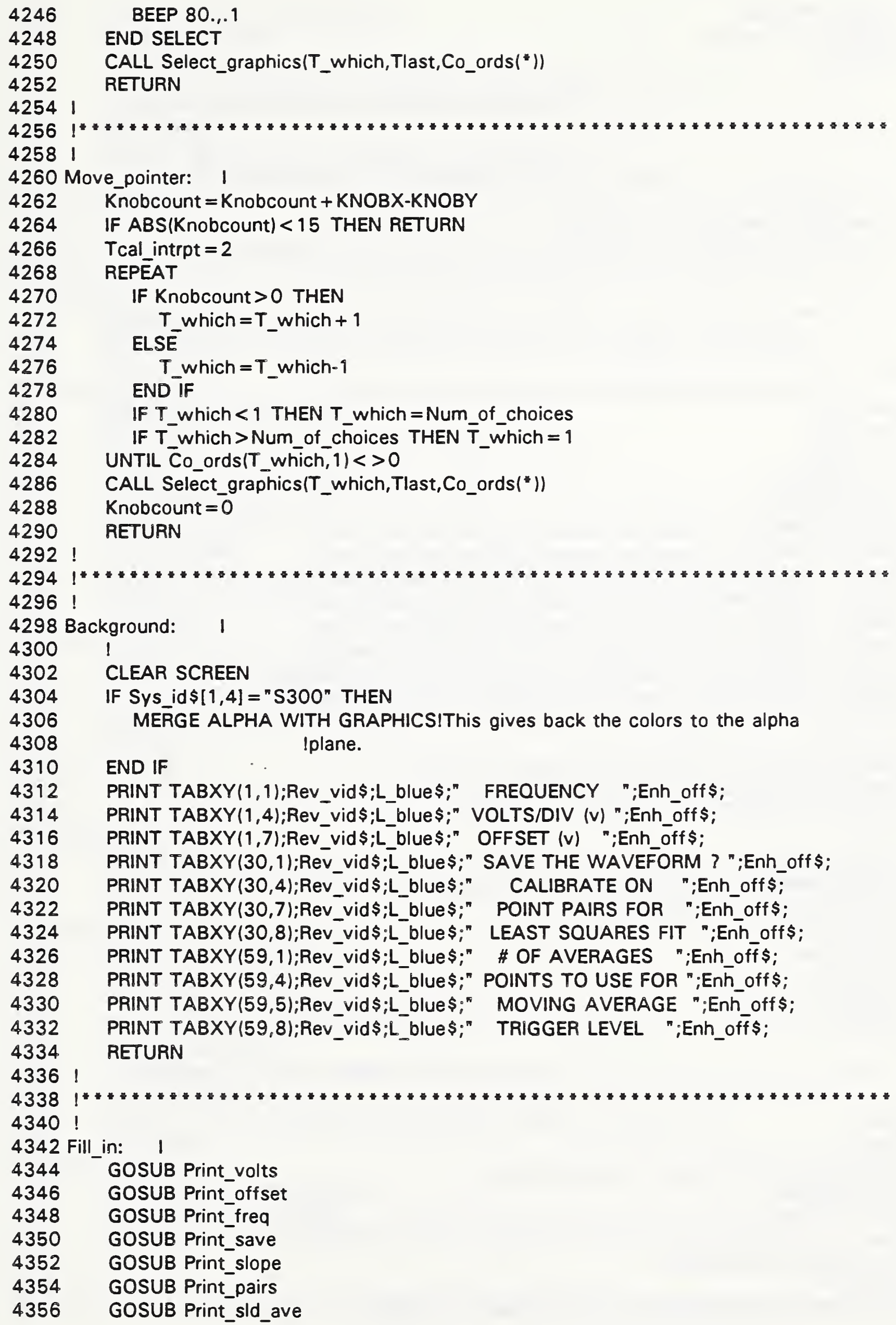


4388 Tcal_set: I

4390

4392

4394

4396

4398

4400

4402

4404

4406

4408

4410

4412

4414

4416

4418

4420

4422

4424

4426

4428

4430

4432

4434

4436

4438

4440

4442

4444

OFF KEY

Ithis allows the operator to manually set up the scope.

OFF KBD

OFF KNOB

Tcal intrpt $=3$

CLEAR SCREEN

PRINT TABXY $(1,1):{ }^{*}$ This is to establish the delay of the time " PRINT " signal only. Any other desired changes will have to be " PRINT " accomplished using the waveform or tcal menus. Use a " PRINT " variable delay line to set the waveform to the desired " PRINT " position. If the delay is set using the DELAY key of the " PRINT " oscilloscope, the time calibration data delay time will be" PRINT " different than the device under test (DUT) waveform delay. " PRINT "Time calibration data is not valid for a DUT waveform " PRINT " which has a different delay time."

!

PRINT TABXY $(1,21): "$ The current channel is "Chnnl

INPUT "is this the correct channel ? YN " $C$ Ch\$

IF $\left(\mathrm{Ch} \$=" n\right.$ ") OR $\left(\mathrm{Ch} \$={ }^{\mathrm{N}} \mathrm{N}\right.$ ) THEN GOSUB Get_chn !

CLEAR SCREEN

PRINT TABXY $(1,24) ;$ :Please set up the waveform and

PRINT "press continue when done."

LOCAL 707

CALL Pause_key_on

OFF KEY

GOSUB Tcal_scope

RETURN

4446

4448

4450 ।

4452 Chnl_err: !

4454 BEEP

4456 DISP "Input out of range of disallowed value."

4458

4460

4462

WAIT Paused

chn:

4464

4466

DISP "Try again ..."

4468

ON ERROR GOTO Chnl_ePr

Test $\$=" m$

INPUT "Enter the acquisition channel number $(1-4) .{ }^{\circ}$,Test\$ 
4472 Temp $=$ VAL(Test $\$$ )

4474 OFF ERROR

4476 IF (Temp < 1) OR (Temp > 4) THEN GOTO Chnnl_error

4478

4480

4482

4484

4486

4488 !

4490 !

4492 !

4494 Volterr: !

4496 GOSUB Beeps

4498 DISP "ERROR IN READING VALUE OR VALUE OUT OF RANGE, TRY AGAIN."

4500 WAIT Paused

4502 Input volts: 1

4504 ONN ERROR GOSUB Volterr

4506 Test\$ $=" n$

4508 INPUT "Enter the volts per division in volts ..." ,Test\$

4510 IF LEN(Test \$) $<1$ THEN RETURN

4512 GOSUB Data_check

4514 IF (Rtemp<(Ätten .001)) OR (Rtemp> (Atten*.080)) THEN GOTO Volter

4516 Tc_volt $=$ Rtemp

4518 Tc_vrange $=$ Tc_volt $* 8$

4520 OUTPUT 707;":CHANNEL" \&VAL\$(Chnnl) \&":RANGE "\&VAL\$(TC_vrange)

4522 OFF ERROR

4524 Print volts:

4526 PRINT TABXY(1,5);Orange\$;

4528 CALL Auto_format(Tc_volt)

4530 IF NOT (Tcal_intrpt) THEN CALL Select_graphics(T_which,Tlast,Co_ords("))

4532 RETURN

4534 !

4536

4538 !

4540 Offset_err: !

4542 GOSUB Beeps

4544 DISP "ERROR IN READING VALUE OR VALUE OUT OF RANGE, TRY AGAIN."

4546 WAIT Paused

4548 Input offset: $\quad$ !

4550 ON ERROR GOTO Offset_err

4552 Test $\$=^{\text {* }}$

4554 INPUT "Enter the offset in volts ..." ,Test\$

4556 IF LEN(Test\$) $<1$ THEN RETURN

4558 GOSUB Data_check

4560 IF ABS(Rtemp) $>5.00 E-1$ THEN GOTO Offset_err

4562 Tc_off $=$ Rtemp

4564 OUTPUT 707:":CHANNEL" \&VAL\$(Chnnl)\&":OFFSET "\&VAL\$(TC_off)

4566 OFF ERROR

4568 Print offset:!

4570 PRINT TABXY(1,8);Orange\$:

4572 CALL Auto_format(TC_off)

4574 IF NOT (Tcal_intrpt) THEN CALL Select_graphics(T_which,Tlast,Co_ords( $\left.{ }^{*}\right)$ )

4576 RETURN

4578 ।

4580 
$4582 !$

4584 Choose_average: 1

4586 OFF KEY

4588 OFF KNOB

4590 OFF KBD

4592 CLEAR SCREEN

4594 GCLEAR

4596 PRINT L_blue $\$$

4598 DIM Dp\$[80]

4600 DIM T\$[52]

4602 Dp $\$=$ "Select Average "

$4604 T \$=$ "Available Averages (powers of 2 ) "

4606 Intr_prty $=$ Intr_prty +3

4608 CALL Menu_scroll(Dp\$,T\$,Allowd\$("), 12,1, What("))

4610 Intr_prty $=$ intr_prty-3

4612 IF What (1) $<>0$ THEN ! Aborted

4614

4616

4618

4620

4622

4624

4626

T_aver $=$ VAL(Allowd $\$($ What $(1)))$

END IF

Tcal_intrpt $=3$

l

OUTPUT @Scope:" :ACQUIRE:TYPE AVERAGE

4628 Print_ave:

4630

4632

4634

4636

4638

4640 !

4642 Save_wave: !

4644

4646

4648

4650

4652

PRINT TABXY(63,2); Orange\$:

CALL Auto_format(T_aver"1.0)

RETURN

4654 Print save:

4656 PRINT TABXY(36,2); Orange\$:

4658 PRINT Save\$

4660 IF NOT (Tcal_intrpt) THEN CALl Select_graphics(T_which,Tlast,Co_ords(" ))

4662

$4664 !$

4666

4668 !

4670 Freq_error: 1

4672 BEEP

4674 DISP "ERROR FREQUENCY INPUT, TRY AGAIN."

4676 WAIT Paused

4678 Input_freq:!

4680 ON ERROR GOTO Freq_error

4682 Test $\$=$ "

4684 INPUT "Enter the calibration frequency..." ${ }^{\circ}$,Test\$

4686 IF LEN(Test \$) < 1 THEN RETURN

4688 GOSUB Data_check

4690 OFF ERROR

4692 IF Rtemp<0 THEN GOTO Freq_error 
$4694 \quad$ Freq $=$ Rtemp

4696 Print_freq:

4698 PRINT TABXY(2,2); Orange\$;

4700 CALL Auto_format(Freq)

4702 IF NOT (Tcal_intrpt) THEN CALL Select_graphics(T_which,Tlast,Co_ords (")1)

4704 RETURN

$4706 !$

4708

4710 !

4712 Pair_error: !

4714 GOSUB Beeps

4716 DISP "ERROR IN READING VALUE OR DISALLOWED VALUE, TRY AGAIN."

4718 WAIT Paused

4720 Point_pairs: !

4722 ON ERROR GOTO Pair_error

4724 Test $\$=$ " $\mathrm{m}$

4726 DISP "Enter the point pairs for the linear least squares fit."

4728 WAIT Paused

4730 INPUT " This must be a positive, integer number." Test\$

4732 IF LEN(Test\$) $<1$ THEN RETURN

4734 Temp $=$ VAL (Test\$)

4736 OFF ERROR

4738 IF Temp $<1$ THEN GOTO Pair_error

4740 Ls_prs $=$ Temp

4742 Print_pairs: I

4744 PRINT TABXY(35,9);Orange\$;

4746 CALL Auto_format(Ls_prs*1.0)

4748 IF NOT (Tcal_intrpt) THEN CALL Select_graphics(T_which,Tlast,Co_ords(*)|

4750 RETURN

4752 ।

$4754 !$

4756 ।

4758 Which_slope: I

4760 IF Slope THEN

4762 SIpe $\$={ }^{*}$ - SLOPE "

4764

4766

4768

4770

4772

$$
\text { Slope }=0
$$

ELSE

Slpe $\$="+$ SLOPE ${ }^{n}$

Slope $=1$

4774 Print

4774 Print_slope: I

4776 PRINT TABXY(35,5);Orange\$;

4778 PRINT SIpe\$

4780 IF NOT (Tcal_intrpt) THEN CALL_Select_graphics(T_which, Tlast,Co_ords ( $*$ ))

4782 RETURN

4784 !

4786

4788 !

4790 Moving_error: !

4792 GOSUB Beeps

4794 DISP "ERROR IN READING VALUE OR DISALLOWED VALUE, TRY AGAIN."

4796 WAIT Paused

4798 DISP "This must be an odd, positive integer number."

4800 WAIT Paused

4802 Moving_points: !

4804 ON ERROR GOTO Moving_error 


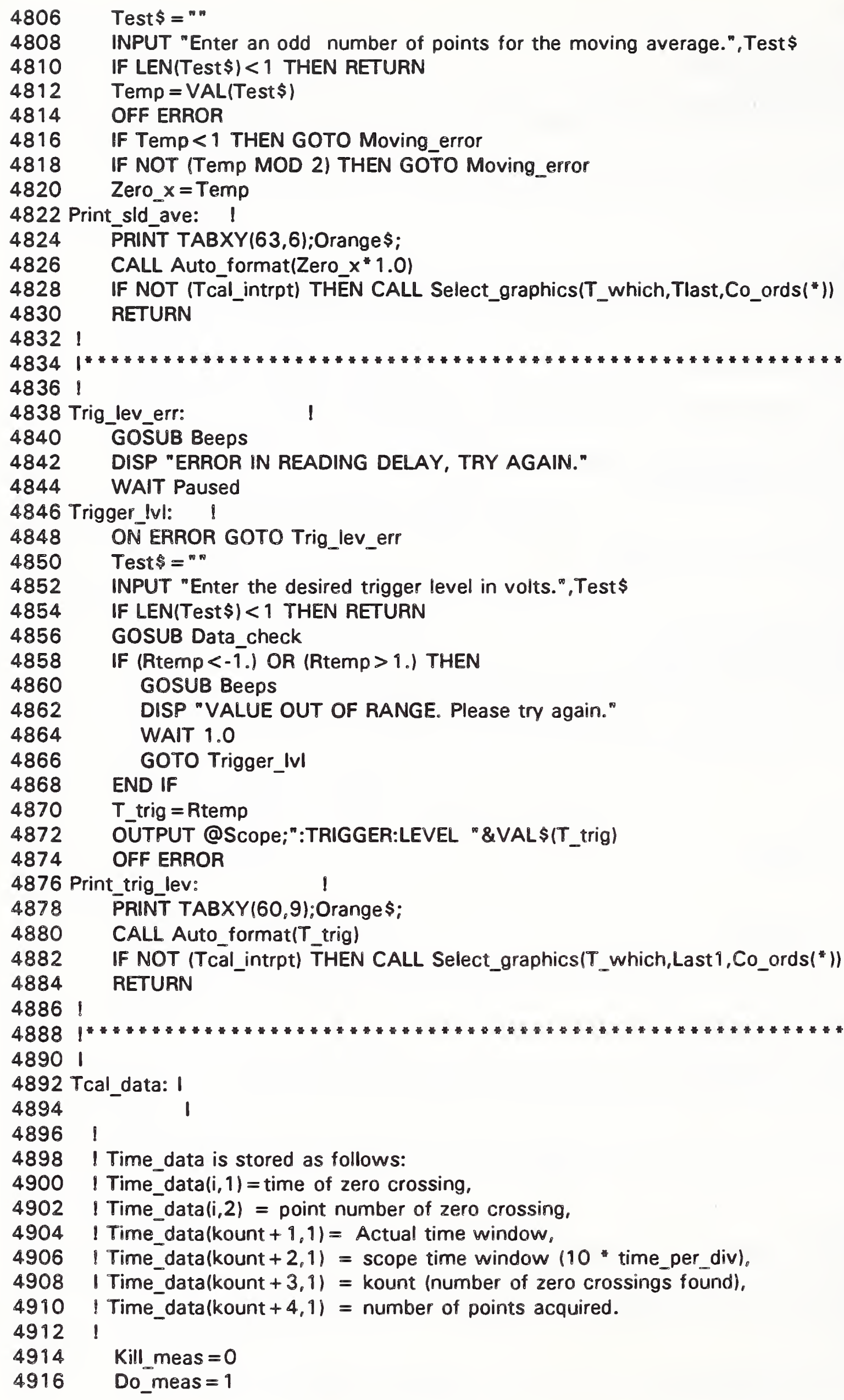


5008

5010 5012 !

5014 5016 ! 5018 Abort tmeas: !

OFF KEY

OFF KBD

OFF KNOB

END IF

\section{ELSE}

END IF

\section{END IF}

OFF KEY

RETURN

Kill_meas $=1$
WHILE NOT (Kill_meas) AND (Do_meas)

ON KEY O LABEEL "ABORT ",Local_prty + 5 GOTO Abort_tmeas

IF Do meas THEN GOSUB Get_twave

END WHILLE

IF NOT (Kill meas) THEN GOSUB Con_to_volts GOSUB Sliding_ave GOSUB Linear_fit GOSUB Crossing_point

IF Save $\$=$ " YES "THEN

DISP "A description of the time calibration waveform required;" WAIT Paused INPUT "enter data description, 40 chrs or less. ",Data_id\$ Intr_prty = Local_prty +1

CALL Data_to_disk_r(1,INT(Pnts),Voltage("),Data_id\$) Intr_prty = Local_prty

\section{OFF KEY}

\section{CLEAR SCREEN}

PRINT TABXY $(1,1)$;" There were ";Time_data(Kount + 3,1);

PRINT "zero crossings and ";Time_data(Kount $+4,1)$;" points acquired."

PRINT "The time window as calculated against the time standard ";

PRINT "is ";Time_data(Kount $+1,1) ; "$ ".

PRINT "The time window that the oscilloscope reports is ";Time_data(Kount $+2,1) ;$ "."

INPUT "Is the data Okay? $y / n$ ", Ch\$

IF $\mathrm{Ch} \$=$ " $n$ " OR Ch $\$=$ "N" THEN

DISP "Please re-set the measurement and try again."

WAIT 1.5

CLEAR @Scope

DISP "Enter in the description of the time ";

INPUT "calibration data, 40 characters or less. "Data_id \$ Intr_prty = Local_prty +1

CALL Data_to_disk_r( $(9, K o u n t+4$,Time_data ("),Data_id $\$)$ Intr_prty = Local_prty

\section{DEALLOCATE Voltage(")}

DEALLOCATE XCross(")

DEALLCCATE Time data(")

DEALLOCATE Xpnts(*)

TCal_intrpt $=3$

\section{CLEAR @Scope}

LOCAL @Scope

CLEAR SCREEN

DISP Red\$;"MEASUREMENT ABORTED" 


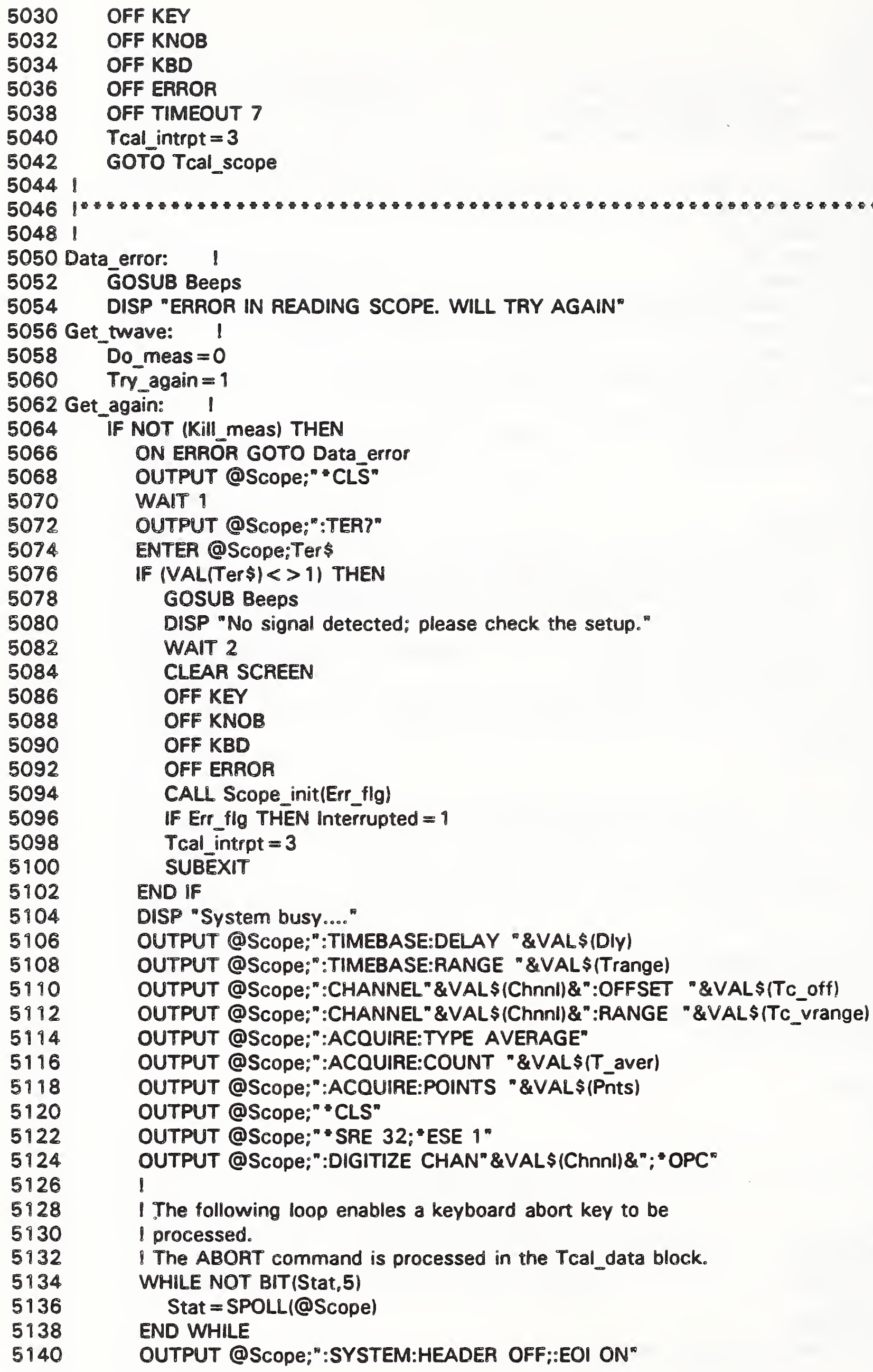


5236 Con_to_volts: !

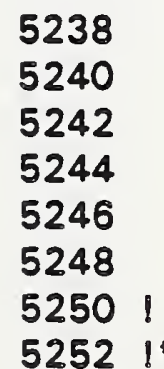

5238

5240

5242

5244

5246

5248

$5250 !$

5252

OUTPUT @SCOpe;"WAVEFORM:SOURCE WMEMORY" \&VAL\$(Chnnl)\&"; FORMAT WORD" OUTPUT @Scope;"WAVEFORM:DATA?"

ENTER @Scope USING "\#,A,D";Header\$,Bytes

IF Bytes $=3$ THEN

ENTER @Scope USING "\#,3D";Length

END IF

IF Bytes $=4$ THEN

ENTER @Scope USING "\#,4D";Length

END IF

Length $=$ Length $/ 2$

IF Pnts $<>$ Length THEN

DISP "The scope will not allow ",Pnts, "points."

WAIT Paused

DISP "The number of points is now ",Length,"."

WAIT Paused

Pnts = Length

END IF

REDIM Wav(Length)

ENTER @Scope USING "\#,W";Wav(")

ENTER @Scope USING "-K,B";End\$

OUTPUT @SCOPe;":WAVEFORM:YINCREMENT?"

ENTER @Scope;Yinc

OUTPUT @Scope;":WAVEFORM:YORIGIN?"

ENTER @Scope;Yorg

OUTPUT @Scope;" :WAVEFORM:YREFERENCE?"

ENTER @Scope;Yref

OUTPUT @SCOPe;":WAVEFORM:XINCREMENT?"

ENTER @Scope;Xinc

OUTPUT @Scope;":WAVEFORM:XORIGIN?"

ENTER @Scope;Xorg

OUTPUT @Scope;":WAVEFORM:XREFERENCE?"

ENTER @Scope;Tref

OFF ERROR

Bug $1=1$

IF Bug 1 THEN

FOR I $=1$ TO Pnts

PRINTER IS PRT

PRINT Wav(l)," ",I

NEXT I

PRINTER IS CRT

Bug $1=0$

END IF

END IF

RETURN 
5256 Sliding_ave: I

5258 ALLOCATE REAL Xcross(100)

5260 ALLOCATE Xpnts(Pnts)

5262 Kount $=0$

5264

5266

5268

5270

5272

5274

5276

5278

5280

5282

5284

5286

5288

5290

5292

5294

5296

5298

5300

5302

5304

5306

5308

5310

5312

5314

5396

5318

5320

5322

5324

Int_temp $=$ Zero_ $\times$ DIV 2

GOSUB Mid

Zero_level $=($ Midval $* 1.0) *($ Zero_ $x * 1.0)$

Slide_ave $=0$. Zero_level

FOR $\bar{l}=1$ TO Zero_ $x$

Xpnts $(l)=1$

Slide_ave $=$ Slide_ave + Wav (I)

NEXT I

FOR $1=$ Zero_ $x+1$ TO Pnts

Xpnts $(l)=1$

Prev_ave $=$ Slide_ave

Slide_ave $=$ Slide_ave + Wav (I)-Wav (ll-Zero_ $x)$

IF ( Prev_ave"Slide_ave<0) OR (Slide_ave $=0 \|$ THEN

$I$ a change in sign indicates a zero crossing.

1

INegative slope crossing

1

IF (NOT Slope) AND (ISlide_ave-Prev_ave) $<\approx 0.1$ THEN

Kount $=$ Kount +1

Xcross (Kount) $=\mid-$ Int_temp

END IF

1

IPositive slope crossing

I

IF (Slope) AND ((Slide_ave-Prev_ave) $>=0$. ) THEN

Kount $=$ Kount +9

Xcross (Kount) $=$ I-Int_temp

END IF

END IF

NEXT I

RETURN

5326

5328

$5330 !$

5332 Mid: !

$5334 \quad$ Maxval $=$ Wav $(9) * 1.0$

$5336 \quad$ Minval $=$ Wav(1) 1.0

5338 FOR $I=1$ TO Pnts

5340

5342

5344

5346

5348

53501

5352

5354 !

5356 Crossing_point: 1

5358

5360

5362

If $($ Wav $(I) * 1.0)>$ Maxval THEN Maxval $=\operatorname{Wav}(1) * 1.0$

IF $($ Wav $(1) * 1.0)<$ Minval THEN Minval $=$ Wav $(1) * 1.0$ NEXT I

Midval $=$ INT $($ (Maxval + Minval $) / 2)$

RETURN

5364 I Time_data is stored as follows: 
( Time_data(i,1) = time of zero crossing,

( Time_data(i,2) = point number of zero crossing,

5432 Linear_fit: I

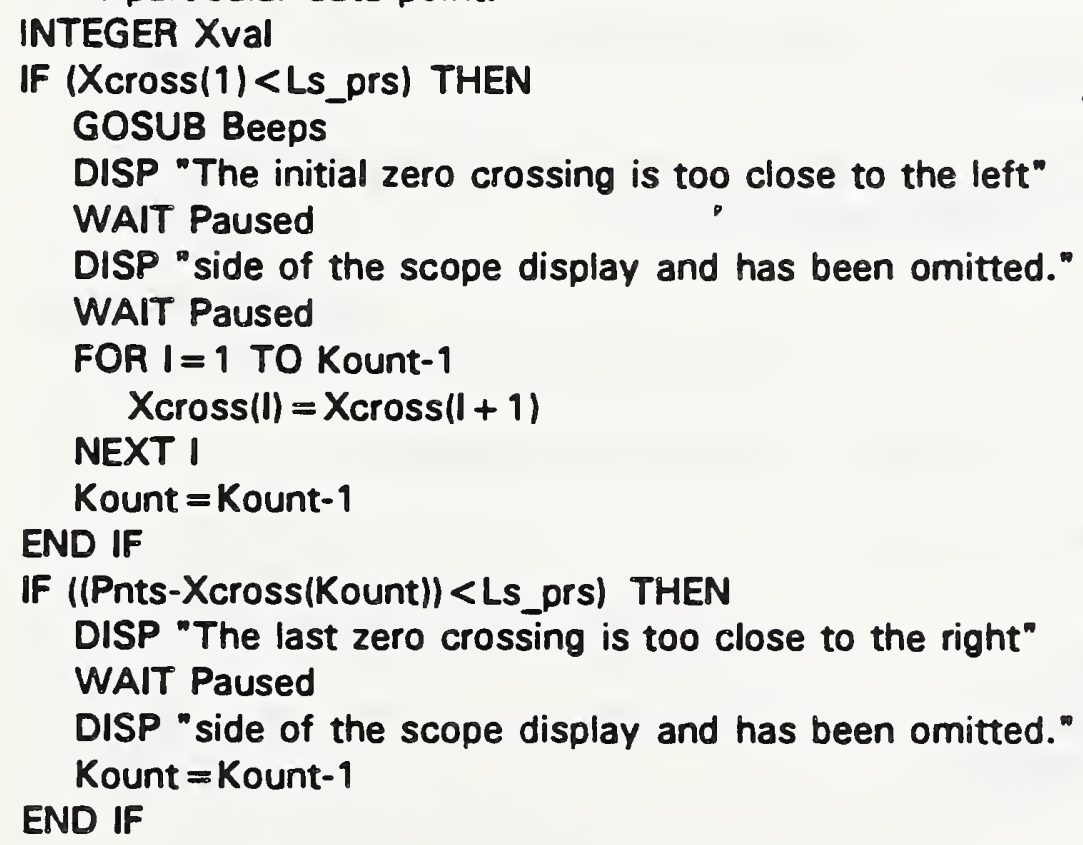




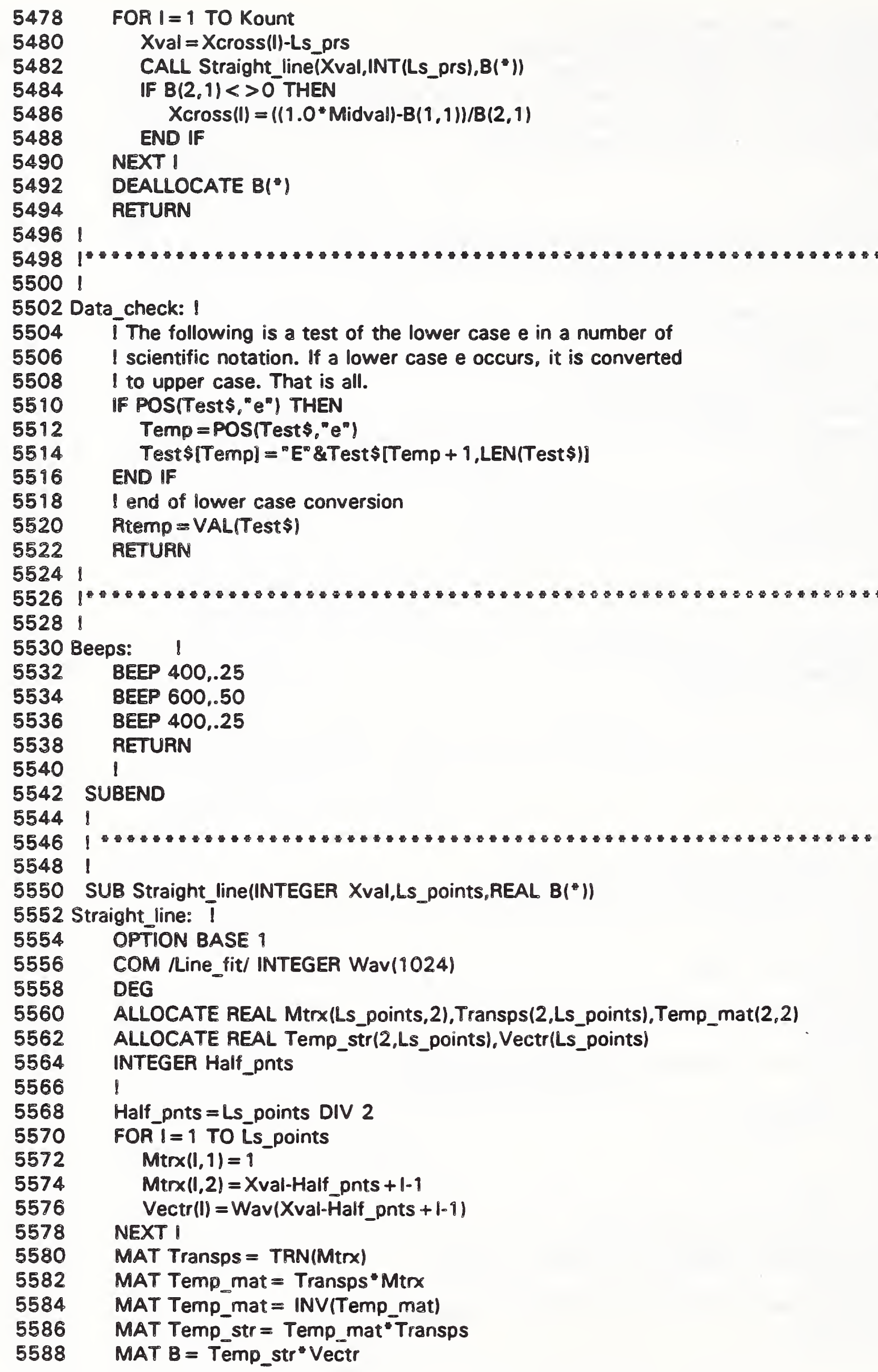




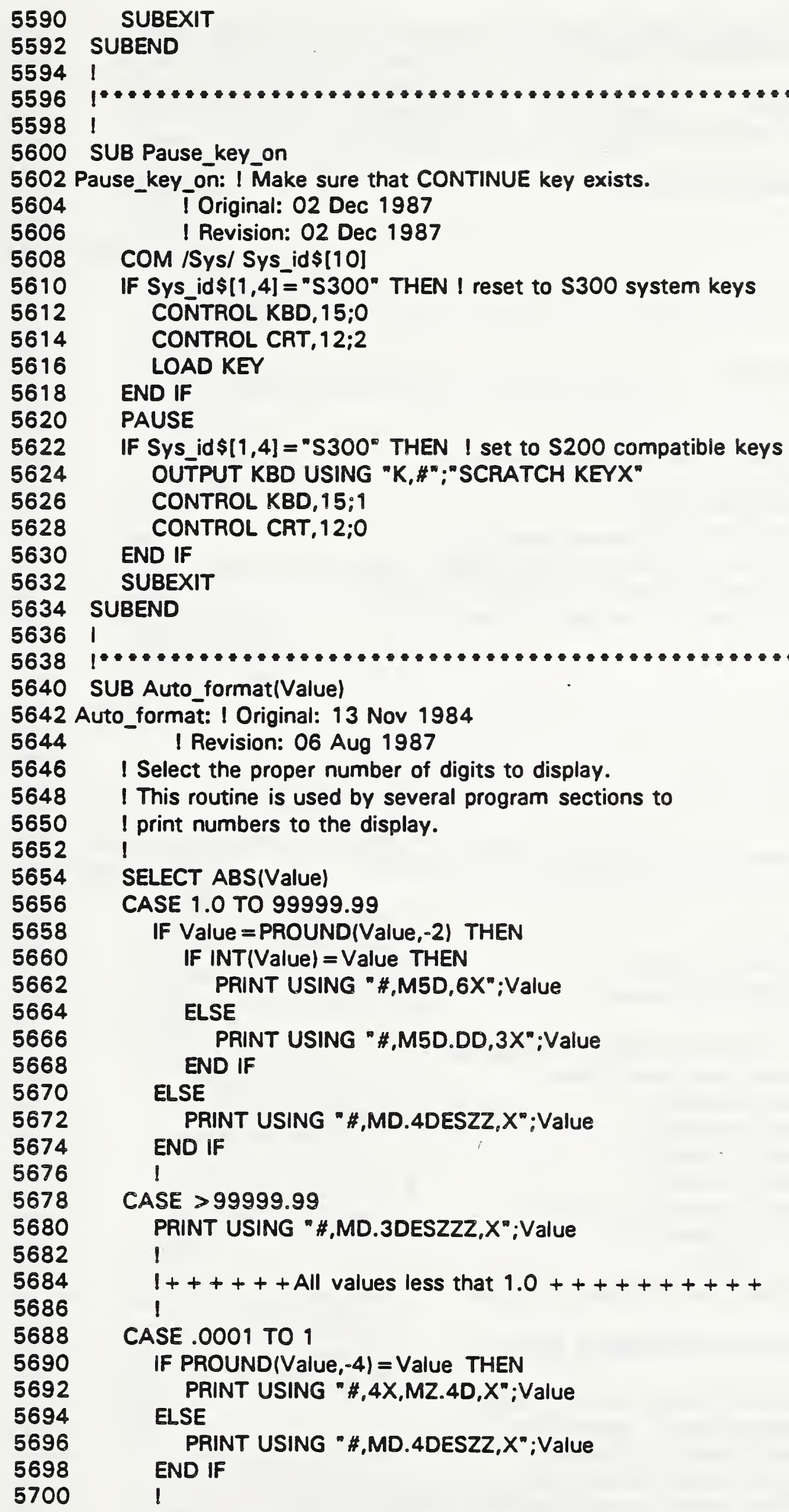


5760 Init_epr:

5762

5764

5766

5768

5770

5772

5774

5776

5778

5780

5782

5784

5786

5788

5790

5792

5794

5796

5798

5800

5802

5804

5806

5808

5810

5812

1 1 1

l
CASE $1.0 E-99$ TO .0001 PRINT USING " \#,MD.4DESZZ, $X^{n}$;Value 1

CASE 1.0E-300 TO 1.0E-99 PRINT USING " \#,MD.3DESZZZ, $X$ ";Value

CASE ELSE PRINT USING "\#,4X,MZ.D,4X";Value

END SELECT

SUBEXIT

SUBEND

SUB Scope_init(INTEGER Err_flg)

Scope_init: $!$

COM /Scope/ REAL Time_per_div,Volts, Trange,Vrange,Diy

COM /Scope/ REAL Probe_fac,Offs, Trig, Atten

COM /Scope/ INTEGER Aver,Pnts,Chnnl

COM /Scope/ Type\$[30],Refer\$[14],@Scope,Mode\$[30]

COM $\pi$ cal_vals/ INTEGER Zero_ $x_{\text {, }}$ Ls_prs,Slope, $T_{-}$aver,REAL Freq

COM Tral vals/ Sipe $\$ 1101$, Save $\$[10]_{0}$ REAL TC_off,TC_volt, T_trig

COM Ncal_vals/ INTEGER V_aver, Interval, REAL $V_{\infty}$ step, $V_{-\infty}$ min

Erp $f l g=0$ ino errors yet.

Try_again $=1$

GOS̄UB Do_again

SUBEXIT

BEEP

DISP "Something went wrong during initialization. Will try again."

WAIT 1.5

Do_again: !

ON ERROR GOTO Init_err

ON TIMEOUT 7,2 GOTO TIME_out_error

CLEAR 707 Iclears the HPIB to the scope

ASSIGN @Scope TO 707

OUTPUT @Scope; ${ }^{\text {"RST" }}$ "Puts the scope in a known state.

IThe rest puts the scope in a more useful state.

OUTPUT @SCOPe:"BLANK CHANNEL 9"

OUTPUT @Scope ${ }^{\circ}$ BLANK CHANNEL2"

OUTPUT @SCOPe;"BLANK CHANNEL3"

OUTPUT @SCOPe;"BLANK CHANNEL4"

OUTPUT @SCOPe:" :DISPLAY:GRATICULE GRID"

OUTPUT @SCOpe:" :DISPLAY:FORMAT 1"

IF Refer $\$=$ " CENTER "THEN

OUTPUT @SCOPe;"TIMEBASE:REFERENCE CENTER"

ELSE

OUTPUT @Scope;"TIMEBASE:REFERENCE LEFT"

END IF

OUTPUT @Scope:" :TIMEBASE:RANGE \& \& VAL\$(Trange)

OUTPUT @Scope:":TIMEBASE:DELAY " \&VAL\$(Dly)

OUTPUT @SCOpe:":TIMEBASE:MODE "\&MOde\$

OUTPUT @SCOpe;"VIEW CHANNEL"\&VAL\$(Chnnl)

OUTPUT @Scope;":CHANNEL" \&VAL\$(Chnnl)\&":OFFSET "\&VAL\$(Offs) 
5874 SUB Select_graphics (INTEGER Which,Last1,Co_ords (")।

5876 Select_graphics:

$5924 \quad$ LwrlftX $=$ LwrlftX-2 


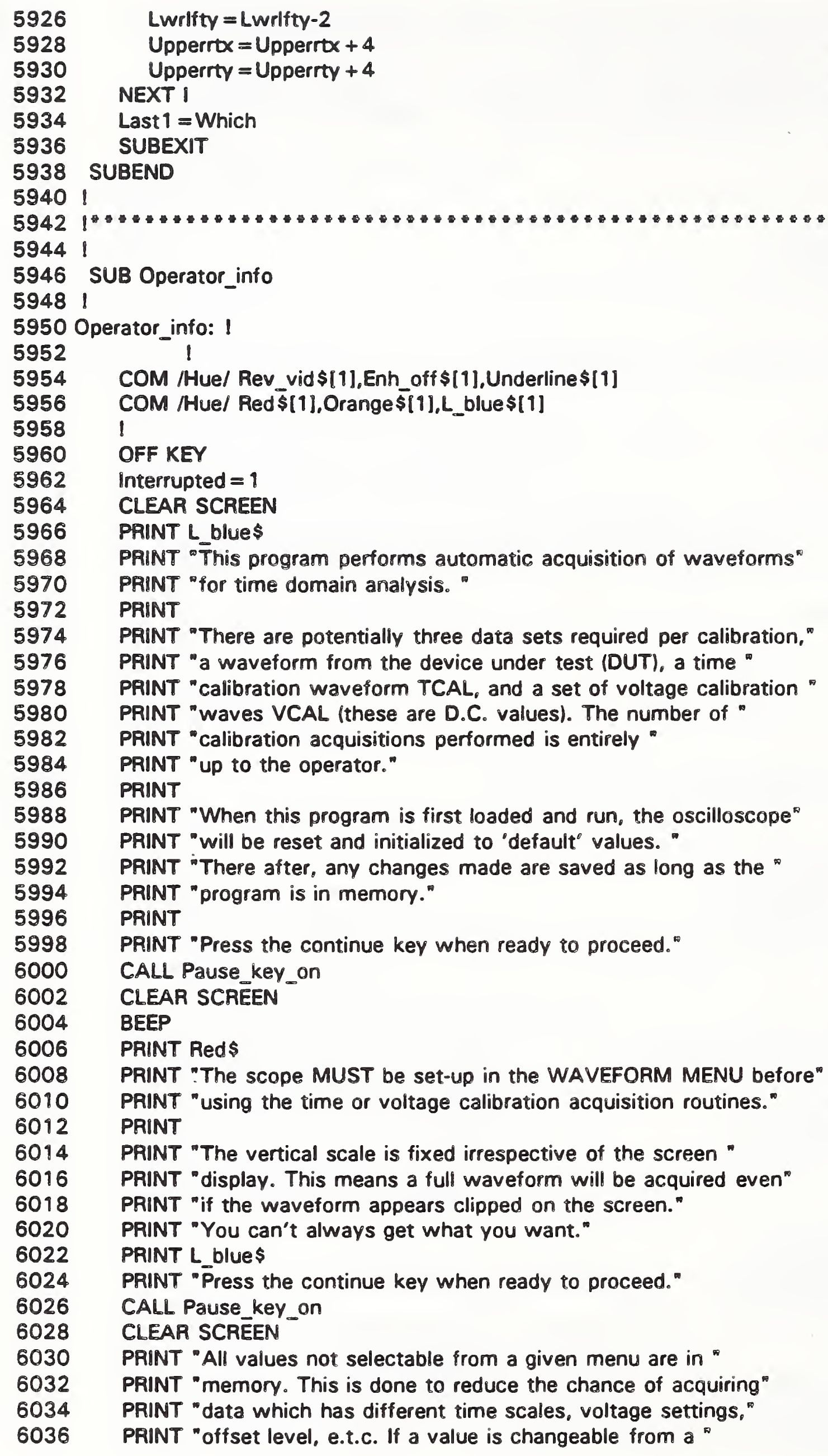

5962

5964

PRINT "When this program is first loaded and run, the oscilloscope PRINT "will be reset and initialized to "default" values." PRINT "There after, any changes made are saved as long as the " PRINT "program is in memory." PRINT

PRINT "Press the continue key when ready to proceed."

CALL Pause_key_on

CLEAR SCREEN

BEEP

PRINT Red\$

PRINT "The scope MUST be set-up in the WAVEFORM MENU before PRINT "using the time or voltage calibration acquisition routines." PRINT

PRINT "The vertical scale is fixed irrespective of the screen " PRINT "display. This means a full waveform will be acquired even" PRINT "if the waveform appears clipped on the screen." PRINT "You can't always get what you want." PRINT L_blue\$ PRINT "Press the continue key when ready to proceed." CALL Pause_key_on CLEAR SCREEN

PRINT "All values not selectable from a given menu are in " PRINT "memory. This is done to reduce the chance of acquiring" PRINT "data which has different time scales, voltage settings," PRINT "offset level, e.t.c. If a value is changeable from a " 
PRINT "menu then it does not need to be consistent with the " PRINT "other waveforms. For example, the voltage offset level "

6080 SUB Data_to_disk_r(INTEGER Curve,Datacount,REAL Basket_file("),Data_id\$)

6082 Data_to_disk_r: I Original: 13 Nov 1984

6084 TRevision: 02 Dec 1987

6086 IThis routine will SAVE data files on the disk in RAW data format.

6088 OPTION BASE 1

6090 COM /Files/ Diskdrive $\$[20]$,Filename $\$[14]$,Ms_path $\$[500]$

6092 COM Interrupts/ INTEGER Intr_prty

6094 INTEGER Local_prty,Diskspace

6096 DIM Ac $\$[5]$, Status $\$[1]$, Tempfile $\$[14]$

6098 REAL Dtime

6100 OFF KEY

6102 Local_prty = Intr_prty

$6104 \quad$ Dtime $=0$.

$6106 \quad 1$

6108 ISelect the disk drive for data storage

6110

6112 Selectdrive: 1

6114 GRAPHICS OFF

6116 OUTPUT 2 USING ${ }^{n} \#, K^{n} ;{ }^{n} K^{n}$

6118 CALL Select_disk

6120 IF Diskdrive $\$=$ "NO DISK" THEN GOTO Mistakeline

6122 Choosefilename: I

$6124 \quad A C \$=$ "ABORT"

6126 Tempfile $\$=$ Filename $\$$

6928 CALL Enterfilename(AC\$)

6130 IF LEN(Filename $\$$ ) $=0$ THEN

6132 Filename $\$=$ Tempfile $\$$

6134

6136

GOTO Mistakeline

END IF

6138 Send_to_disk: ! Create file and save information.

6140 ŌN ERROR GOTO Cant_savedata

6142 Diskspace $=$ INT $(($ Datacount $* 16.0) / 256)+2$

6144 CREATE BDAT Ms_path \$\&Filename \$\&iskdrive\$,Diskspace,256

6146 Dtime $=$ TIMEDATE

6148 DISP " SAVING data for CURVE \# ";Curve;". " 

ASSIGN @Datapath TO Ms_path\$\&Filename \$\&Diskdrive\$ OUTPUT @Datapath;Status $\$$ OUTPUT @Datapath;Data_id\$ 140 chrs description if single curve. OUTPUT @Datapath;Datacount Inumber of xy points OUTPUT @Datapath;Datacount Isize of array (same as above) OUTPUT @Datapath;Basket_file( $($ )

6166

6168

6170 Mistakeline:OFF KEY

6172

6174

6176

6178

6180

6182

6184

6186

6188

6190 Cant_savedata: !

6192

6194

6196

6198

6200

6202

6204

6206

6208

6210

6212

6294

6216

6218

6220

6222

6224

6226

6228

6230

6232

6234

6236

6238 SUBEND ASSIGN @Datapath TO.

OFF ERROR

!

LOOP

EXIT IF TIMEDATE-Dtime $>1.8$

END LOOP

DISP CHR\$ (12)

OUTPUT 2 USING "\#,K";"K"

SUBEXIT

I

[ /IIIIIIIIIIIIIIIIIIIIIIIIIIIIIIIIIIIIIIIIIIIIIIIIIIIIIII

$!$

BEEP 500.6

SELECT ERAN

CASE $72,73,76.78,81,82.90,93$

DISP Diskdrive $\$_{:}^{\circ}$ has failed of is not available *:

DISP *...CONTINUE to try again."

CALL Pause_key_on

Filename $\$=$ Tempfile $\$$

CASE 84,85

DISP " This disk is not initialized ":

DISP "...CONTINUE to try again."

CALL Pause key_on

Filename $\$=$ Tempfile $\$$

CASE 55,64

DISP " This disk is full, insert new floppy and/or":

DISP " select new drive ...CONTINUE "

CALL Pause key_on

Filename $\$=\bar{T}$ emprile $\$$

CASE ELSE

CALL Errortrap

GOTO Send_to_disk

END SELECT

GOTO Selectdrive

6240

6242

6244

6246 SUB Select_disk

6248 Select_disk: I Original: 13 Nov 1984

6250

6252

I Revision: 02 Dec 9987

6254

6256

6258

OPTION BASE 1

COM /Files/ Diskdrive $\$[20], F i l e n a m e \$[14], M s \_p a t h \$[500]$

COM /Interrupts/ INTEGER intr_prty

6260

COM /Sys_msi/Msi_id\$

COM /Sys/ Sys_id\$ 
I Define the disk drives available for this system, reserve the

I first characters for the drive address and the characters after 1

$\operatorname{Disc} \$(D d+1)={ }^{*}:, 702,0$

Disc $\$(D d+2)=":, 702,1$

Disc $\$(D d+3)=":, 703,0$

Disc $\$(D d+4)=":, 1400$
- HP 9122 dual microfloppy left drive"

- HP 9122 dual microfloppy right drive"

- HP 9125 single 5.25 floppy drive"

- HP 9133H hard disk volume 1 " 

$D d=D d+4 \quad l$ add the number of drive specifiers above 1 IF Sys_id $\$[1,4]<>$ "S300" THEN Disc $\$(D d+1)={ }^{m}: 4,9 \quad$ - LEFT internal series $200^{\circ}$

6384

6386

6388

6390

6392

6394

$$
D d=D d+2
$$

\section{END IF}

6400

6402

6404

6406

6408

6410

6412

6414

6416

6418

6420

6422

6424

6426 Diskselected:OFF KEY

6428

6430

6432

6434

6436

6438

I $\quad$ [

1

Intt_prty $=$ Local_prty +1

CALLL Menu_scroll(Displ\$,Title\$,Disc\$("),Dd,Pt,Choose ("))

Intr_prty = Local_prty-1

IF $P$ t $=0$ THEN

ELSE

Diskdrive $\$=$ "NO DISK"

Dd $=$ POS (Disc $\$($ Choose $(P t)), "=")-1$ I find .

IF Dd $>5$ THEN \& valid msus ELSE

Diskdrive $\$=\operatorname{TRIM} \$(\operatorname{Disc} \$(\operatorname{Choose}(P t))[1, D d])$

DISP " ERROR in reading MSUS from string, - chr not found. "

BEEP

CALL Pause_key_on

END IF

Diskdrive $\$=$ "NO DISK"

END IF

6440 Enterfilename: IOriginal: 13 Nov 1984

6442

6444

6446

6448

6450

6452

6454

6456

6458

6460

6462

6464

6466

6468

6470

6472

6474

6476

6478

6480

6482

6484

\section{SUBEXIT}

SUBEND

!

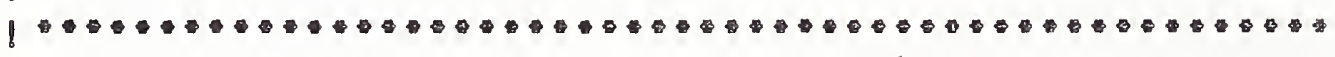

1

SUB Enterfilename(Ac\$)

\& Revision: 10 Dec 1990 includes HFS directories OPTION BASE 1

COM /Files/ Diskdrive\$[20],Filename\$[14],Ms_path\$[500]

COM /Interrupts/ INTEGER Intr_prty

INTEGER 1,Ascii_num,Maskflag,Namelength

DIM Test\$[256],Hfs_temp\$[161]

Namelength $=10$

IF LEN(Ms_path\$) >0 THEN OUTPUT KBD USING "K, \#";" \#" \&Ms_path\$\&" $H^{n}$ DISP " ENTER HFS directory PATH (no file)":

IF AC\$ $<>$ "PATH" THEN

DISP ". ENTER / for HFS ROOT or null for LIF...":

END IF

LINPUT His_temp\$

His_temp\$ $=$ TRIM\$(Hfs_temp\$)

IF LEN(Hfs_temp\$1>0 THEN

IF LEN(Hfs_temp $\$$ ) $>1$ AND Hfs_temp\$[LEN(Hfs_temp $\$ 1: 9]<>\% /^{\circ}$ THEN Hfs_temp $\$=$ Hfs_temp $\$ Q^{m} /{ }^{\circ}$

END IF

IF LEN(Hfs_temp $\$)=1$ THEN Hfs_temp $\$={ }^{\omega}$

Namelength $=14$

END IF

IF AC\$ $=$ "PATH" THEN 


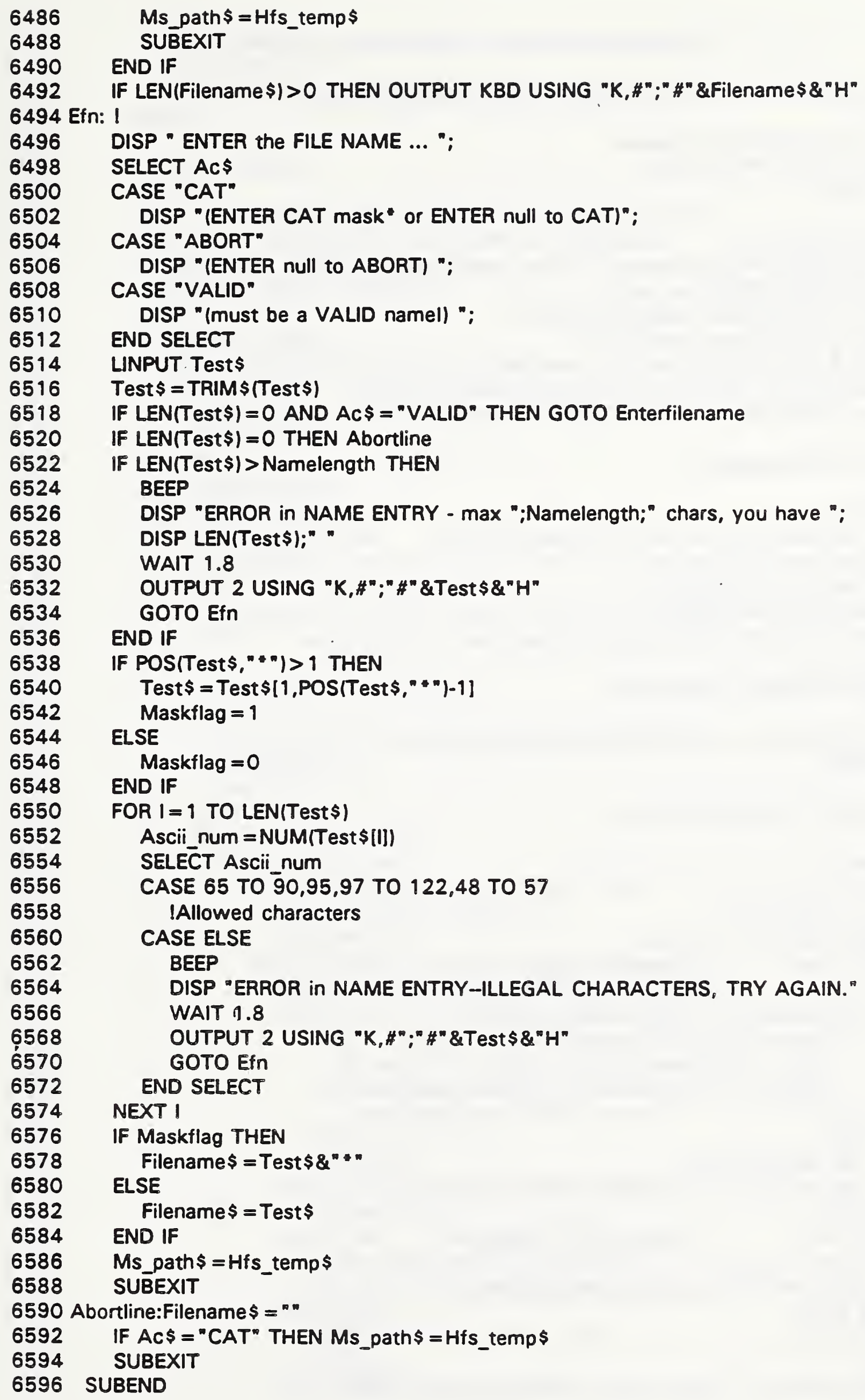


6604 SUB File_menu(Mask\$,Ftype\$,Fis\$("I,INTEGER Fis_ent,Dir_on,Prt_on)

6606 File_menu: I

6608 I Original: 29 Jun 1987, G. Koepke

6610 I Revision: 02 Dec 1987, 07:00

6612 OPTION BASE 1

6694 DEG

6696 COM/Sys/ Sys_id\$[10] COM /Files/ Diskdrive\$[20],Filename\$[14],Ms_path\$[500] COM Anterrupts/ INTEGER Intr_prty COM /Bugs/ INTEGER Bug1,Bug2,Bug3,Printer DIM Directory $\$(600)[80], B d \$(600)[71]$ DIM D\$[80],T\$[51],Ids\$[40],Stat\$[1],Test $\$[256]$ INTEGER Bd_cnt,File_ent,I,C_cnt,CO(1),Format_error,End_search IF Fis_cnt $>0^{-}$THEN ÁLLOCATTE INTEGER Choose(Fis_cnt)

End search $=0$

REPEAT \& Generate path to file and extract file name.

ON ERROA GOTO Cat errors

6644

DISP " Reading the Directory ...

IF LEN(MS path $\$$ ) $>0$ THEN

MASS STORAGE IS Ms_path\$[1,LEN(Ms_path\$)-1]\&Diskdrive \$

6648

6650

6652

6654

6656

6658

6660

6662

6664

6666

6668

6670

6672

6674

6676

6678

6680

6682

6684

6686

6688

6690

6692

6694 ELSE

MASS STORAGE IS Diskdrive\$

END IF

CAT TO Directory $\$(")$ :NO HEADER,COUNT File_cnt OFF ERROR

I

I set up array of legal file names.

is

$B d c n t=0$

MÁT Bd\$ $=(" \bar{n})$

FOR $l=1$ TO File cnt

SELECT Directory $\$(1)[32.36]$

CASE Ftype $\$$ I Ftype $\$=$ "BDAT" or

I Ftype $\$=$ "PROG"

IF LEN(Mask\$) >0 THEN ! Test for mask \$

IF Directory $\$(1)[9$, LEN(Mask $\$)]=$ Mask $\$$ THEN

$\mathrm{Bd} \_\mathrm{cnt}=\mathrm{Bd} \_\mathrm{cnt}+1$

Bd $\$\left(B d \_c n t\right)=$ Directory $\$(1)[1 ; 14] \& "$ - "\&Ftype $\$$

END IF

ELSE

Bd_cnt $=$ Bd_cnt +1

$B d \bar{s}\left(B d \_c n t\right)=$ Directory $\$(1)[1: 14] \& "$ - "\&Ftype $\$$

END IF

6696

6698

6700

6702

6704

6706

6708

CASE "DIR " I plus all "DIR " listings

$\mathrm{Bd} \mathrm{cnt}=\mathrm{Bd} \mathrm{cnt}+1$

Bd\$ $($ Bd_ent $)=$ Directory $\$(1)[1 ; 14] \&^{\infty}$ - DIR *

CASE ELSE

END SELECT

NEXT I

IF LEN(Ms_path $\$$ ) $>0$ AND Bd_cnt $>0$ AND Fls_cnt $>0$ THEN 
$B d \_c n t=B d \_c n t+1$

Bd $\overline{\$}\left(B d_{-}\right.$cnt $)="$ - MOVE back up ONE Directory level." 
6822

6824

6826

6828

6830

6832

6834

6836

6838

6840

6842

6844

6846

6848

6850

6852

6854

6856

6858

6860

6862

6864

6866

6868

6870 Read

6872

6874

6876

6878

6880

6882

6884

6886

6888

6890

6892

6894

6896

6898

6900

6902

6904

6906 NOL reCOg ND SELECT

6908

6910

6912

6914

6916

6918

6920

6922

6924

6926 Go_on:NEXT I

6928

6930

6932

$$
\text { NEXT I }
$$

END IF

ELSE

WAIT 2.5

End_search $=9$

END IF

DISP CHR\$ 112)

UNTIL End_search

SUBEXIT

Cerrors:!

BEEP

CALL Pause_key_on

DISP CHR\$(12)

C_ent $=0$

MAT FIS $\$=(" n)$

SUBEXIT

!

!

SELECT Stat\$

CASE "N"

CASE "Y"

END SELECT

OFF ERROR

END IF GOTO Go_on

Other format:!

END IF

DISP CHR\$(12);

RETURN

$$
I=C_{-} \text {cnt }
$$

END IF

DISP " This directory contains no ":Ftype $\$_{0}^{* 1}$ files ..."

DISP " ERROR ... ":ERRM\$

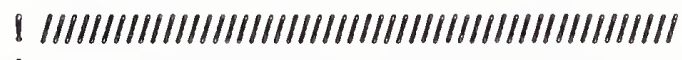

d_data_id: I This routine expects to see Ids $\$$ from

I GRAPH_DATA raw data files.

DISP " Reading file contents ... Please stand by. "

PRINT TABXY $(1,18) ;$; Reading \#":

FOR $l=1$ TO Bd_cnt $\mid$ each BDAT file

PRINT TABXȲ $(19,18)$ :

PRINT USING "3D,4A,3D,2A, \#", 10 " of "Bd_cnt ${ }_{0}{ }^{\circ}$ 。"

Ids $\$=$ "Data not recognized."

IF Bd\$(1) [18, 22] = "BDAT " THEN

ON ERROR GOTO Not_recognized

ASSIGN @lo_path TO Bd\$(I) $[1 ; 14]$

ENTER @lo_path:Stat\$

ENTER @lo_path;lds\$

Ids $\$=$ "Complete graph in GRAPH_DATA form."

IF Dir_ON $=2$ THEN

GŌSUB Interpret_1

IF Format_error THEN GOTO Other_format

$\operatorname{Bd} \$(1)[23,71]={ }^{\circ} \ldots{ }^{\circ}$ \&lds $\$$

PRINT TABXY $(1,18):$ RPT $\$\left({ }^{\circ} * 40\right)$; 
6940 Interpret $1: 1$ This is used to interpret ID strings.

6942 Format_error $=1$

6944 I identify this particular format

6946 RETURN

6948

6950

6952

6954 List_directory: I This routine will provide a tabular listing of

6956

6958

6960

6962

6964

6966

6968

6970

6972

6974

6976

6978

6980

6982

6984

6986

6988

6990

6992

6994

6996

6998

7000

7002

7004

7006

7008 Printep kaput:DISP " Printer not pesponding ... listing aborted. "

7010 BEEP

7012 WAIT 9.8

7014 OFF TIMEOUT 7

7016 RETURN

7018 SUBEND

7020

7022

7024

7026 SUB Menu_scroll(D\$,T\$,Items \$( $)$,INTEGER Item_cnt,To_select,Choose (*)|)

7028 Menu_scroll:! Original: 22 Jun 1987, Galen Koepke, NBS $\overline{7} 23.04$ 
1 the user may access screens via softkeys. There may be

I up to 40 screens or 600 items to choose from.

( Maximum sizes: $D \$[80], T \$[51]$, Items $(*)[70]$

1 Items $\$\left({ }^{*}\right)$ contains the item descriptions

I Item_cnt is the number of items in Items $\$(1)$

( Choose $($ ) is dimensioned to the number of required choices

$! \quad$ and will be filled with the item numbers chosen.

I To_select is the number of required choices.

1

OPTION BASE 9

PRINTER IS CRT

DEG

GOSUB Def_variables

GOSUB Define_screens

GOSUB Make_selections

IF Null file THEN ! reset to zero

Item_cnt $=0$

Items $\$(1)=n$ "

To_select $=0$ in no valid selections

SUBEXIT

\&

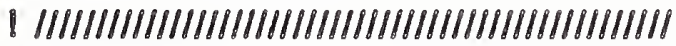

1

ef_variables:!

COM /Interrupts/ INTEGER Intr_prty

COM /Bugs/ INTEGER Bug 1,Bug2,Bug3,Printer

COM /Sys/ Sys_id\$110]

!

INTEGER Screen_cnt,Items_per_scn,First_item(40),Last_item(40)

7908 INTEGER I,J,K,First_line, Last_line,Active_screen,Pointer, Last_pt

7910

7912

7914

7996

9198

7920

7122

7124

7126

7928

7130

7132

7134

7136

7138

7940

7142

7944

7146

7148

7950

7952

7154 INTEGER Local_prty,SKips,Knobcount,Pointeractive,KO,Null_file INTEGER Exit_flag,Temp,Random_select,Indx

DIM Marker \$18],Test\$[256]

I

I initialize parameters

I

Local_prty $=$ Intt_prty

IF Local_prty $<1$ THEN Local prty $=10$

IF LEN(SYS_id\$) $=0$ THEN SYS id\$ = SYSTEM\$("SYSTEM ID")

IF Item_cnt $<$ ? THEN

Null_file $=1$

Item_ent $=$ ?

To_select $=0$

Items $\$(1)=$ Empty

ELSE

Null_file $=0$

END IF

IF To_select $=-1$ THEN

Random_select $=1 \quad 1$ choose random number of items

To select $=0 \quad$ i needed for softkeys

END IF

IF To_select $>$ Item_cnt THEN To_select $=1$ tem_cnt

MAT Choose $=$ (9999)

$7156 \quad$ Knobcount $=0$ 
1

7170 Define_screens:! Set up screens of 15 items each.

Items_per_scn $=15$ I Maximum number of displayable items

IF INT(Item_cnt/items_per_scn) =Item_cnt/ltems_per_scn THEN ELSE Screen_cnt $=$ INT (Item_cnt/ltems_per_scn)

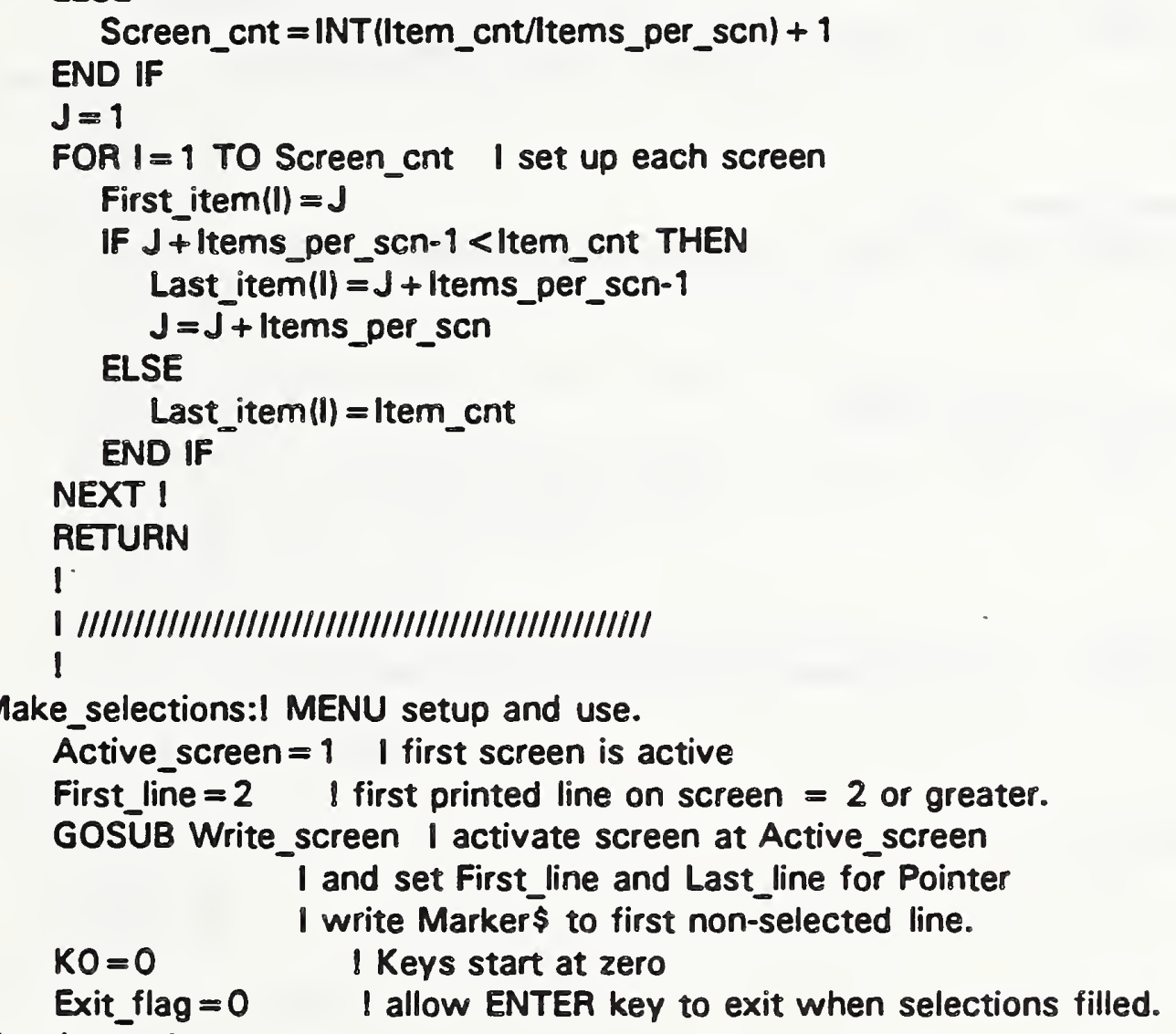


7408 Jump_to_errors:DISP " Not a valid screen number ... try again."

7410

7412 1

! IIIIIIIIIIIIIIIIIIIIIIIIIIIIIIIIIIIIIIIIIIIIIIII

WAIT 9.8

7414 Jump_to_scn: !

7416 OFF KBD

7418 OFF KNOB

7420 OFF KEY

7422

7424

DISP " ENTER the screen number desired $(1$ to ";Screen_cnt:" $) . "$;

LINPUT Test\$

7426 Test $\$=$ TRIM $\$($ Test $\$)$

7428 IF LEN(Test\$) $=0$ THEN Jump_to_return

7430

7432

7434

7436

7438

7440

7442

7444

7446

7448

7450

7452

7454

7456 Select_fixed:!

ON ERROR GOTO Jump_to_errors

Temp = INTIVAL(Test\$))

OFF ERROR

IF Temp $<1$ OR Temp $>$ Screen_cnt THEN Jump_to_errors

Active_screen $=$ Temp

GOSUB Write_screen

7458

7460

7462

7464

7466

7468

7470

7472

7474

7476

7478

7480

7482

OFF KBD

OFF KNOB

OFF KEY

IF NOT Pointeractive THEN

DISP "NO additional selections for this screen."

\section{BEEP}

WAIT 2

DISP CHR\$ (12):

$$
\text { RETURN }
$$

END IF

IF Skips $=$ To_select THEN

IF To_select $=0$ THEN DISP "This menu is for information only,"; DISP " no selection allowed."

\section{ELSE}

DISP "All selections have been filled,":

DISP " 'Select Reset' to repeat."

END IF 


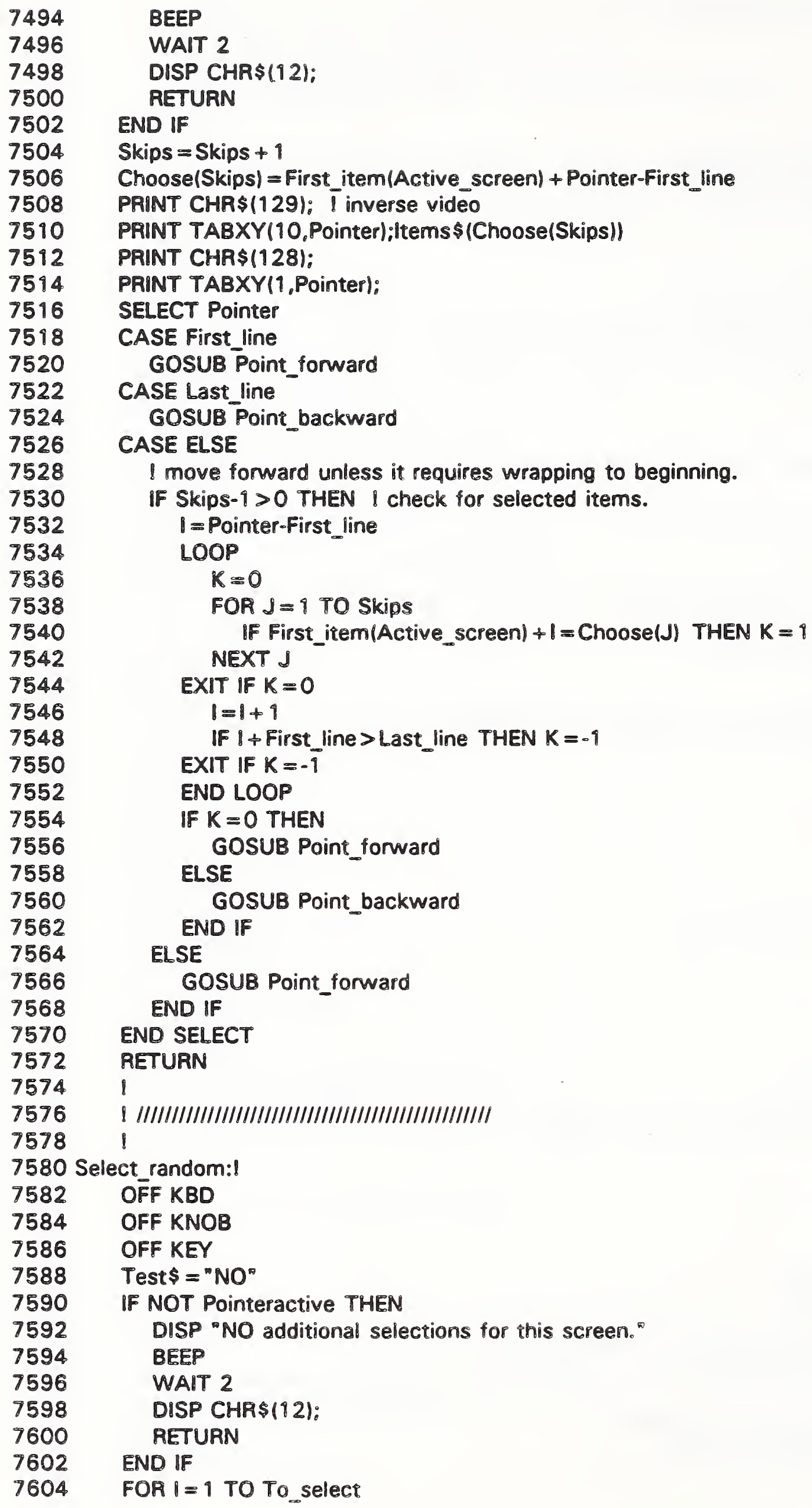


7672

7674 Select_reset: IClear Choose file

7676

7678

7680

7682

7684

7686

7688

7690

7692

7694

7696

7698

7700

7702

7704

7706

7708

7710

7712

7714

7716 Ind $x=1$

Test $\$=$ "YES"

\section{END IF}

\section{NEXT I}

SELECT Test\$

CASE "YES"

\section{ELSE}

\section{END IF}

FOR $I=\operatorname{lndx}$ TO To_select-1

Choose $(I)=$ Choose $(1+1)$

\section{NEXT I}

Choose(To_select) $=999$

To_select $=$ To_select 1

CASE "NO"

To_select $=$ To_select +1

$$
\text { ELSE }
$$

\section{END IF}

\section{END SELECT}

PRINT CHR\$(128);

PRINT TABXY(1,Pointer):

RETURN

I

1

OFF KBD

OFF KNOB

OFF KEY

Skips $=0$

MAT Choose $=$ (999)

GOSUB Write_screen

RETURN

l

!

Test $\$=$ KBD $\$$

BEEP 80...1

RETURN

END IF

SELECT Test $\$[2,2]$

CASE CHR\$(255)
If Choose $(I)=$ First_item(Active_screen) + Pointer-First_line THEN

I Selected item is tagged ... untag

IF Pointer $<>$ Last_item(Active_screen) +1 AND Pointer $<>17$ THEN PRINT CHR\$(1 28$)$; I normal video

PRINT CHR\$(132);I underline video

PRINT TABXY(10,Pointer);Items $\$($ Choose(Ind $x))$

I Selected item is untagged ... tag it

Choose $($ To_select $)=$ First_item (Active_screen) + Pointer-First line

IF Pointer $<>$ Last_item(Áctive_screen) +1 AND Pointer $<>17$ THEN PRINT CHR\$(129);! inverse video

PRINT CHR\$(133); inverse video with underline

PRINT TABXY(10,Pointer);Items\$(Choose(To_select))

! IIIIIIIIIIIIIIIIIIIIIIIIIIIIIIIIIIIIIIIIIIIIIII

IF Random_select THEN TO_select $=0$

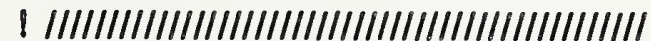

Process_kbd:I Allow use of arrows and enter key in addition to soft.

IF LEN(Test $\$$ ) $=1$ AND Test $\$ 1,1]<>$ CHR $\$(32)$ THEN

IF Test $\$ 1,1]=$ CHR\$(32) THEN GOSUB Point forward

IF Test $\$ 11,1]<>$ CHR\$(255) THEN RETURN 
I do nothing

CASE " $V$ " "T"

GOSUB Point_forward

7722

7724

7726

7728

7730

7732

7734

7736

7738

7740

7742

7744

7746

7948

7750

7752

7754

7756

7758

7760

7762

7764

CASE "A" "W"

GOSUB Point backward

CASE "E" " $" s^{n}, " t^{n}, "{ }^{\prime \prime}$

IF Random_select THEN

GOSUB Select_random

ELSE

IF Skips < To select THEN

GOSUB Sélect_fixed

ELSE

I exit routine

END IF

Exit_flag $=1$

END IF

CASE ELSE

BEEP 80...9

END SELECT

Test $\$=\pi \mathrm{m}$

RETURN

I

I IIIIIIIIIIIIIIIIIIIIIIIIIIIIIIIIIIIIIIIIIIII!

7766 Point forward: Knobcount $=5$

7768 GOSUB Move pointep

7770 RETURN

7772 Point backward:Knobcount $=-5$

7774 ǴOSUB Move_pointer

7776 RETURN

7778 \&

$7780 \quad$ I IIIIIIIIIIIIIIIIIIIIIIIIIIIIIIIIIIIIIIIIIIIII

$7782 \quad 1$

$7784 \mathrm{~J}_{0}$ pointer:! Move the selection pointer on the active screen.

$7786 \quad$ I without regard to selected values

7788 If Knobcount $>0$ THEN I Move forward

$7790 \quad$ Pointer $=$ Pointer +1

7792

7794

7796

ELSE

I Move backward

Pointer $=$ Pointer -9

7998 IF Pointer < First line THEN Pointer = Last line

7800 IF Pointer $>$ Last line THEN Pointer $=$ First_line

7802 RETURN

7804

7806

7808

7810 Move_pointer:! Control pointer to avoid re-selection of items

7812 IF NOT Pointeractive THEN RETURN I No selections to be made.

7814 Knobcount $=$ Knobcount + KNOBX-KNOBY

7816 IF ABS(Knobcount) $<4$ THEN RETURN

$7818 \quad$ Last_pt $=$ Pointer

7820 GOSUB Jog_pointer

7822

7824

7826

If Skips $>0$ THEN

LOOP

$J=$ Pointer-First line

7828

FOR I $=1$ TO Skips 
7878 Write_screen:I Write the screen pointed to by Active_screen

END IF

IF First_item(Active_screen) $+\mathrm{J}=$ Choose(I) THEN $\mathrm{J}=999$ NEXT 1

IF $\mathrm{J}=999$ AND Pointer $=$ Last_pt THEN Pointeractive $=0$

EXIT IF Pointeractive $=0$

IF $J=999$ THEN GOSUB Jog_pointer

EXIT IF $\mathrm{J}<>999$

END LOOP

Knobcount $=0$

OUTPUT KBD;CHR\$(255)\&CHR\$(84); I Bring screen home

IF Last_pt = Last_line THEN PRINT CHR\$(132);

PRINT " ":

IF Pointeractive THEN ! Pointer active

IF Pointer $=$ Last_line THEN

ELSE

PRINT CHR\$(132):

END IF

PRINT CHR\$ (128):

PRINT TABXY(1,Pointer);Marker \$;CHR\$(128);

END IF

RETURN

I

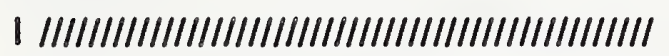

I home and clear screen

OUTPUT KBD;CHR\$(255)\&CHR\$(84)\&CHR\$(255)\&CHR\$(75);

Knobcount $=$ KNOBX-KNOBY $\mid$ Clear knob and keyboard

Knobcount $=0$

Test $\$=$ KBD $\$$

Test $\$=" n$

1

PRINT TABXY(1,First_line-1);CHR\$(132):" Item \# Screen \#";

PRINT USING "\#,2D,4A,2D,3A";Active_screen," of ":Screen_cnt;" | "

PRINT T\$;RPT \$ $"{ }^{\circ} " 59$-LEN(T \$);:

PRINT TABXY(80,First_line-1):" $\left.\right|^{m} ;$ CHR\$(128);

$J=0$

REPEAT

IF $J=$ Last_item(Active_screen)-First_item(Active_screen) THEN

PRINT CHR\$(132):

PRINT TABXY $(1$,First_line + J);RPT $\$("$ ", 80$)$

ELSE

PRINT CHR\$(128):

END IF

PRINT TABXY (5, First line $+J)$;

PRINT USING "3D,A,\#";First_item(Active_screen) + J。" $"$ "

IF Random_select THEN FOR $I=\overline{1}$ TO To_select

IF First_item(Áctive_screen) $+\Omega=$ Choose(l) THEN

END IF PRINT CHR\$(129):

NEXT 1

ELSE

IF Skips $>0$ THEN I make this line inverse video

FOR I $=1$ TO Skips

IF First_item(Active_screen) $+J=$ Choose(I) THEN 
PRINT CHR\$(129):

END IF NEXT I

END IF

END IF

PRINT TABXY(10, First_line + J);items $\$$ (First_item(Active_screen) $+J$ )

PRINT TABXY $(80$, First_line $+J):\left.^{\circ}\right|^{\circ}:$

$j=J+1$

UNTIL $J>=$ (Last_item(Active_screen)-First_item(Active_screen) +1 )

Last_line $=$ Last_item (Active_screen)-First_item(Active_screen)

Last_line $=$ Last_line + First_line

!

I set marker to first non-selected item.

l

Pointeractive $=0$

IF To_select $>0$ OR Random_select THEN Pointeractive $=1$

If Skips $>0$ AND Pointeractive $=1$ THEN $!$ find first non-selected item $J=0$

LOOP

Pointer $=$ First line $+\downarrow$

FOR $I=1$ TO Skips

IF First_item(Active_screen) $+d=$ Choose(l) THEN Pointer $=0$ NEXT I

EXIT IF Pointer $<>0$

$\jmath=J+1$

IF First line $+J>$ Last line THEN

Pointeractive $=0$

Pointer $=$ First

END If

EXIT If Pointer $<>0$

ELSE

Pointer $=$ First_line

END IF

IF Pointeractive THEN

IF Pointer = Last line THEN

PRINT CHR\$ 1932 ):

ELSE

PRINT CHR\$ 198):

END IF

PRINT TABXY(1,Pointer):Marker\$:CHR\$(128):

END IF

RETURN

SUBEND

1 1

SUB Errortrap

Ortrap: I Original: 13 Nov 1984

I Revision: 02 Dec 4987

1 Trap most errors here

OPTION BASE 1

COM /Files/Diskdrive $\$[20], F i l e n a m e \$[14]$,Ms_path $\$[500]$

DIM File $\$[20], T e s t \$[256]$,What $\$[201, A c \$[5]$

BEEP 400,.6

SELECT ERRN 
CASE 54

DISP "DUPLICATE FILE NAME: ";Filename\$;

DISP "....PURGE old one? $(\mathrm{Y} / \mathrm{N})^{n}$;

LINPUT What\$

What $\$=$ TRIM $\$$ (What $\$$ )

SELECT What $\$ 1,1]$

CASE " $Y$ " " $Y$ "

PURGE Ms_path\$ \& Filename \$ \&Diskdrive \$

CASE ELSE

AC $\$=$ "VALID"

CALL Enterfilename(AC\$)

END SELECT

CASE 52,53

DISP "Improper FILE NAME - ENTER NEW FILE NAME";

OUTPUT 2 USING "\#,K,K";"\#";Filename\$

8080

8082

8084

8086

8088

LINPUT Filename

Filename $\$=$ TRIM $\$$ (Filename $\$$ )

CASE 56

DISP "FILE: ";Filename $\$ ; "$ is not on this disk, please insert":

DISP " correct disk"

CALL Pause_key_on

CASE 64

DISP "This disk is full, PLEASE insert clean disk"

CALL Pause_key_on

CASE 56

DISP "DATA INPUT disk must be in drive!! ";

DISP "...CONTINUE when ready."

CALL Pause_key_on

CASE $72,73,7 \overline{6}$

DISP Diskdrive\$;

DISP " is not available, type correct";

DISP " unit specifier (ie. ":,707,0').";

OUTPUT 2 USING "K,\#":Diskdrive\$

LINPUT Diskdrive\$

CASE 80

DISP "CHECK DISK drive door!"

CALL Pause_key_on

CASE ELSE

DISP ERRM\$;" "CONTINUE' when fixed"

CALL Pause_key_on

END SELECT

DISP CHR\$(12)

SUBEXIT

SUBEND

8142

8144

8146 
8006

8008

8010

8012

8014

8016

8018

8020

8022

8024

8026

8028

8030

8032

8034

8036

8038 Errortrap: I Original: 13 Nov 1984

8040

8042

8044

8046

8048

8050

8052

END IF

NEXT I

END IF

END IF

$J=J+l$

Last_line = Last_line + First_line

I

I

Pointeractive $=0$ $J=0$

LOOP

Pointer $=$ First line $+J$

FOR I = $q$ TO Ś Kips NEXT I

EXIT If Pointer $<>0$

$J=J+1$

Pointeractive $=0$

END IF

Pointer $=$ First_line

EXIT IF Pointer $<>0$

ELSE

END LOOP

END IF

IF Pointeractive THEN

If Pointer $=$ Last_line THEN PAINT CHR\$ (132):

ELSE PRINT CHR\$(128):

END IF

END IF

RETURN

SUBEND

!

!

SUB Errortrap

I Revision: 02 Dec 1987

1 Trap most errors here

OPTION BASE 1

BEEP 400,.6

SELECT ERRN
PRINT CHR\$(129):

PRINT TABXY(10,First_line + J):Items\$(First_item(Active_screen) + J)

PRINT TABXY(80, First line $+J)\left._{i}{ }^{\circ}\right|^{\circ:}$

UNTIL $J>=($ Last_item(Active_screen)-First_item(Active_screen) +1 )

Last_line $=$ Last_item(Active_screen)-First_item(Active_screen)

I set marker to first non-selected item.

IF To_select $>0$ OR Random_select THEN Pointeractive $=1$

IF Skips $>0$ AND Pointeractive $=1$ THEN $\mid$ find first non-selected item

IF First item(Active_screen) $+d=$ Choose(l) THEN Pointer $=0$

IF First line $+J>$ Last line THEN

PRINT TABXY(1,Pointer);Marker\$;CHR\$(128);

COM /Files/ Diskdrive $\$[20$ ], Filename $\$$ [14],Ms path $\$[500$ ]

DIM File \$[20],Test\$[256],What\$[20],Ac\$[5] 
8072

8074

8076

8078

8080

8082

8084

8086

8088

8090

8092

8094

8096

8098

8100

8102

8104

8106

8108

8110

8112

8114

8116

8118

8120

8122

8124

8126

8128

8130

8132

8134

8136

8138

8140

8142

8144

8146
CASE 54

DISP "DUPLICATE FILE NAME: ";Filename\$;

DISP "....PURGE old one? (Y/N)";

LINPUT What\$

What $\$=$ TRIM $\$$ (What $\$$ )

SELECT What $\$[1,1]$

CASE " $Y ", " Y$ "

PURGE Ms_path $\$$ \&Filename $\$$ \&Diskdrive $\$$

CASE ELSE

AC $\$=$ "VALID"

CALL Enterfilename(Ac\$)

END SELECT

CASE 52,53

DISP "Improper FILE NAME - ENTER NEW FILE NAME";

OUTPUT 2 USING "\#,K,K";"\#";Filename\$

LINPUT Filename\$

Filename $\$=$ TRIM $\$$ (Filename $\$$ )

CASE 56

DISP "FILE: ";Filename $\$ ; "$ is not on this disk, please insert";

DISP " correct disk"

CALL Pause_key_on

CASE 64

DISP "This disk is full, PLEASE insert clean disk"

CALL Pause_key_on

CASE 56

DISP "DATA INPUT disk must be in drivell "; DISP "...CONTINUE when ready."

CALL Pause_key_on

CASE $72,73,7 \overline{6}$

DISP Diskdrive\$;

DISP " is not available, type correct";

DISP " unit specifier (ie. ":,707,0').";

OUTPUT 2 USING "K, \#";Diskdrive\$

LINPUT Diskdrive\$

CASE 80

DISP "CHECK DISK drive door!"

CALL Pause_key_on

CASE ELSE

DISP ERRM\$;" "CONTINUE' when fixed"

CALL Pause_key_on

END SELECT

DISP CHR\$(12)

SUBEXIT

SUBEND

1 


\section{B.2 DECON_NIST}

100 102

104

106

108

110

112

114

116

198

120

122

124

126

128

130

132

134

136

138

140

142

144

146

148

150

152

154

156

958

160

162

164

166

168

170

172

174

176 Decon: !

$178 \quad$ !

$980 \quad 1$

182 DIM Ch\$[1],Data_id \$40],Set_dc\$[1]

184 INTEGER Itemp,Fīg,Extended, $\bar{R}$ esp_ext,Typ,No_fft

186 REAL Rtemp,Sum,Dum, Atten

188 COMPLEX C_temp

190 COMPLEX Sigma,W

$192 \quad 1$

194 RAD

196 OFF KEY

198

200

202

204

206

208

CLEAR SCREEN

$\mathrm{Ch} \$={ }^{\circ "}$

Number $=32767$

Resp_ext $=0$

Extended $=0$

Typ $=0$ 
No_fft $=0$

CAILL Get_data(Wave(*),Dum,Flg)

Rtemp $=$ Delta_ $x$

INPUT "Nahaman-Gans extend the waveform? $y / n$ (default is $n$ )" $\mathrm{Ch} \$$ 
$360 \mathrm{Ng}$ extend: I

366 ! with zeros.

370 I data to be extended.

372

376 I waveform.

378

380

382

384

386

362 I This routine performs the Nahman-Gans waveform extension for step-

364 I like and square-like waveforms, and "pads" impulse-like waveforms

368 I The array "waveform' is a complex data array which contains the

374 I Span is the sum of the beginning and ending values of the

COM /Data_stuff/ INTEGER Number,REAL Delta_x,REAL Strt_time !

IF NO_fft THEN SUBEXIT

if

IF (Extended) AND (NOT Typ) THEN

END IF

CALL Record_data(Extended,Sum)

SUBEND

SUB Ng_extend(COMPLEX Wave/ /,INTEGER TYp)

Yagain: !

INPUT "Extend to a) 2048 or b) 4096 points. Enter letter." Ch\$

SELECT Ch\$

CASE " $a "$ "A"

Ndex $=2048$

CASE " $B$ " "

Ndex $=4096$

CASE ELSE

BEEP

DISP "Error in selection, try again."

WAIT 9.0

GOTO Try_again

END SELECT

END IF

IF TYP $<>1$ THEN

FOR $\mid=1$ TO Number

Ndex $=1+$ Number

Wave(Ndex) $=$ Wave(Number) + Wave( $(9)-$ Wave(l)

NEXT I

Number $=$ Number 2

ELSE

FOR $1=$ Number +9 TO Ndex

Wave $(I)=\operatorname{CMPLX}(0,0)$ 


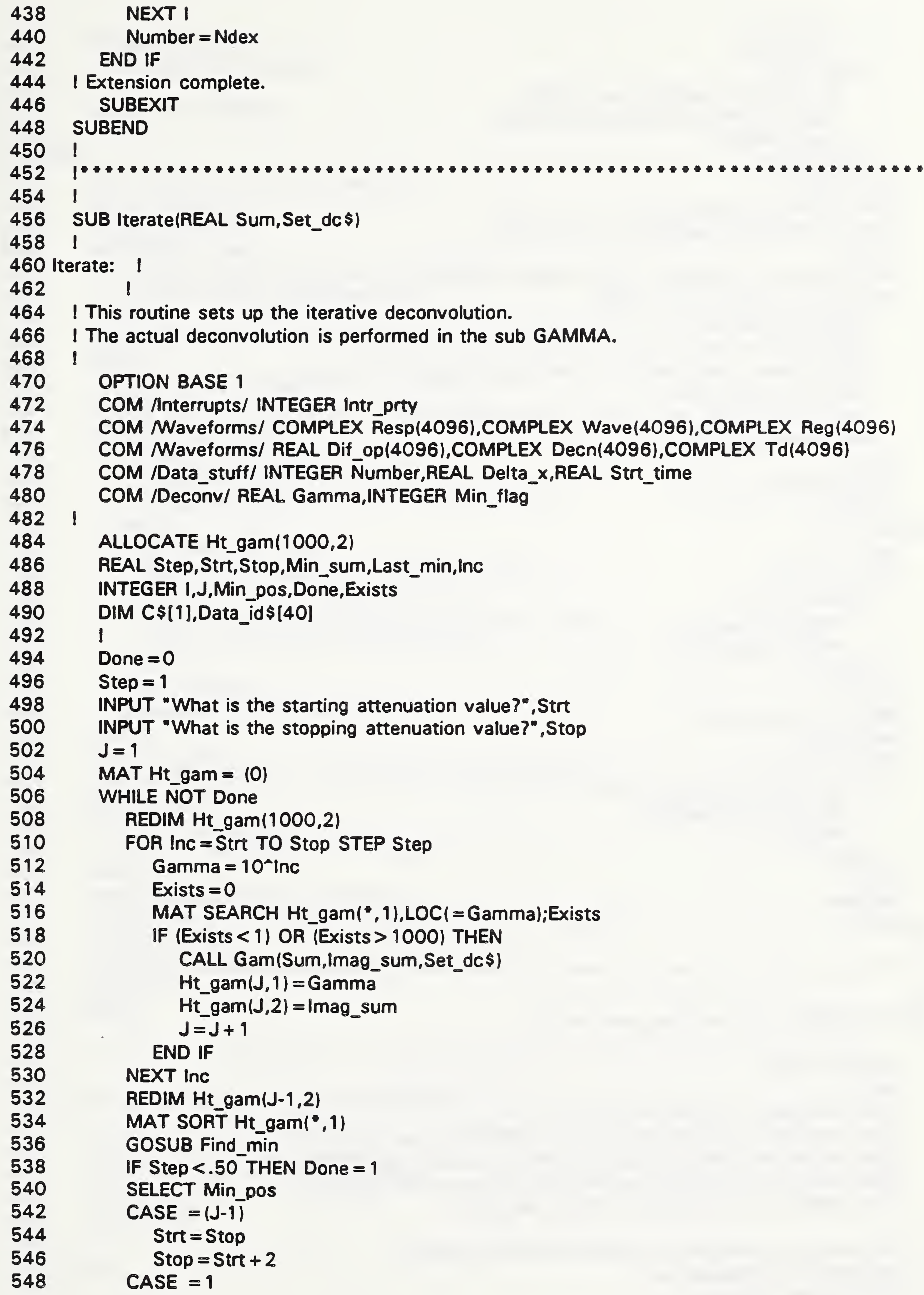




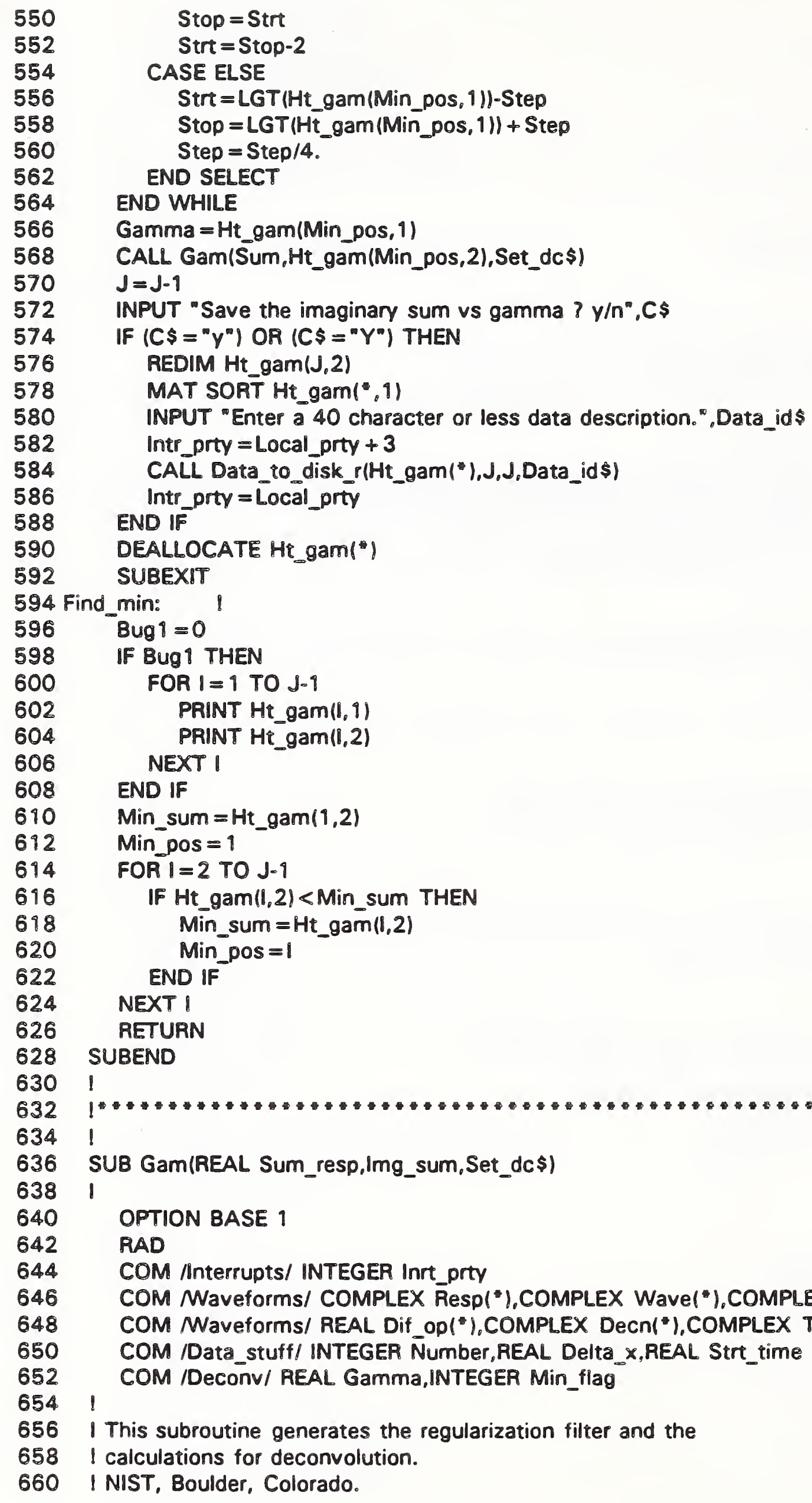

656 I This subroutine generates the regularization filter and the 658 I calculations for deconvolution.

660 I NIST, Boulder, Colorado. 
INTEGER J,No_fft

672 I

674 Gam: I

676 DISP "Performing the deconvolution."

678 Imag_sum $=0$.

REDIM Td(Number)

MAT Decn $=$ Resp

If (Set_dc $\$=" y "$ ) OR (Set_dc $\$=" Y "$ ) THEN

$\operatorname{Decn}(1)=$ CMPLX(ABS(Resp(2)),0.)

686

688

ELSE

IF $\operatorname{Decn}(1)=\operatorname{CMPLX}(0 ., 0$.$) THEN Decn (1)=\operatorname{CMPLX}($ Sum_resp, 0.$)$

692

END IF 
$12=0$

856 SUB Fft_fix(COMPLEX Fft_file(*),INTEGER Err,INTEGER Fft_flg)

$858 \mathrm{Fft}$ fix: IOriginal: $04 \mathrm{Jul} 1987$. J. Ladbury

DISP " Calculating an FFT ... please wait "
Timer = TIMEDATE

Intr_prty $=$ Local_prty +3

CALL Fft_fix(Fft_file("), 12,Fft_flg)

Intr_prty $=$ Local_prty

1 Fft file(") peturns with results, 12 is an error flag.

IF $12<>0$ THEN

SELECT 12

CASE 1

DISP " Negative data count, FFT aborted."

WAIT 1

CASE 2

DISP " Data has zero or negative spacing, FFT aborted."

WAIT 9

\section{CASE 3}

DISP " Data count not a power of 2, FFT aborted."

WAIT 1

CASE ELSE

DISP " ERRORs in FFT, operation aborted."

\section{END SELECT}

DISP "Continue ..."

PAUSE

ELSE

GOTO No_fft_action

\section{SUBEXIT}

\section{END IF}

LOOP \& This keeps the last disp (before the case stmt.) on screen

EXIT IF TIMEDATE-Timer $>1.8$

END LOOP

1

1

DISP "Due to a previously described error or another strange event,"

DISP "no FFT was performed."

No $f f t=9$

SUBBEXIT

SUBEND

!

1

1

1 Revision: 02 Dec 1987

! Modifications: 24 Aug 90 S.M. Chesnut

1 As modified, this routine; checks for valid data and

1 splits the input array (Fft_file) into its real and imaginary

1 parts in preparation for the Fast Fourier Transform (FFT).

1 After the FFT has been performed, the real and imaginary

1 results are stored in the Fft_file array.

OPTION BASE 1

COM /interrupts/ INTEGER ints_prty

COM /Data_stuff/ INTEGER Number,REAL Delta_x,REAL Strt_time 1

1

INTEGER Local_prty,Power 
I

Local_prty $=$ Intr_prty

Err $=\overline{0}$

IF Number $<2$ THEN I gotta have more than one point.

Err $=1$

SUBEXIT

END IF

IF (Delta_ $x<=0$ ) THEN ! Check for positive time.

Err $=\overline{2}$

SUBEXIT

\section{END IF}

Fstep $=1 /$ (Delta_ $x *($ Number $))$

Power $=$ LOG (Number)/LOG $(2)$

IF INT $\left(\left(2^{\wedge}\right.\right.$ Power $\left.)+.5\right)<>$ Number THEN I FFT on data which has I power of 2 data points.

$$
\begin{aligned}
& \text { Err }=3 \\
& \text { SUBEXIT }
\end{aligned}
$$

END IF

ALLOCATE REAL Re(Number), Im(Number)

MAT Re $=$ REAL(Fft_file)

MAT Im = IMAG (Fft_file)

Intr_prty $=$ Local_prty +3

Power $=$ Power +9

CALL Fft_imag(Number*1,,Power*1.0,Fft_flg*1.0,Rel*), Im(*))

Intr_prty = Local_prty

MAT Fft_file = CMPLX $(R e, I m)$

Bug1 $=0$

IF Bug 1 THEN

FOR I = 1 TO Number

NEXT I

PRINT Fft_file(I);I

END IF

DEALLOCATE Re(*), Im(*)

\section{SUBEND}

1

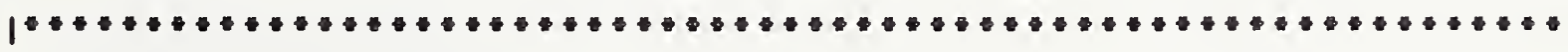

I Algorithm from HP library of math routines.

I Modified 25 October 1990 to work on complex data.

1 Modification by J. Ladbury of NIST, Boulder, Colorado.

\section{OPTION BASE 1}

RAD

Baddta $=(\mathbb{N}<=0)$ OR $(\mathrm{Flg}<>1)$ AND $(\mathrm{Flg}<>-1)$ OR (Power $<=0)$

IF Baddta $=0$ THEN 986

PRINT FNLin $\$(2) ; "$ ERROR IN SUBPROGRAM Fft."

PRINT "N = " $N$, "Flg = ";Flg, "Power = ";Power;FNLin\$ (2)

CALL Pause_key_on GOTO 974

$K=0$

$$
\begin{aligned}
& \text { FOR } J=1 \text { TO } N-1 \\
& I=2 \\
& \begin{array}{l}
I F K<N / I \text { THEN } 1000 \\
K=K-N / I \\
I=I+I
\end{array}
\end{aligned}
$$




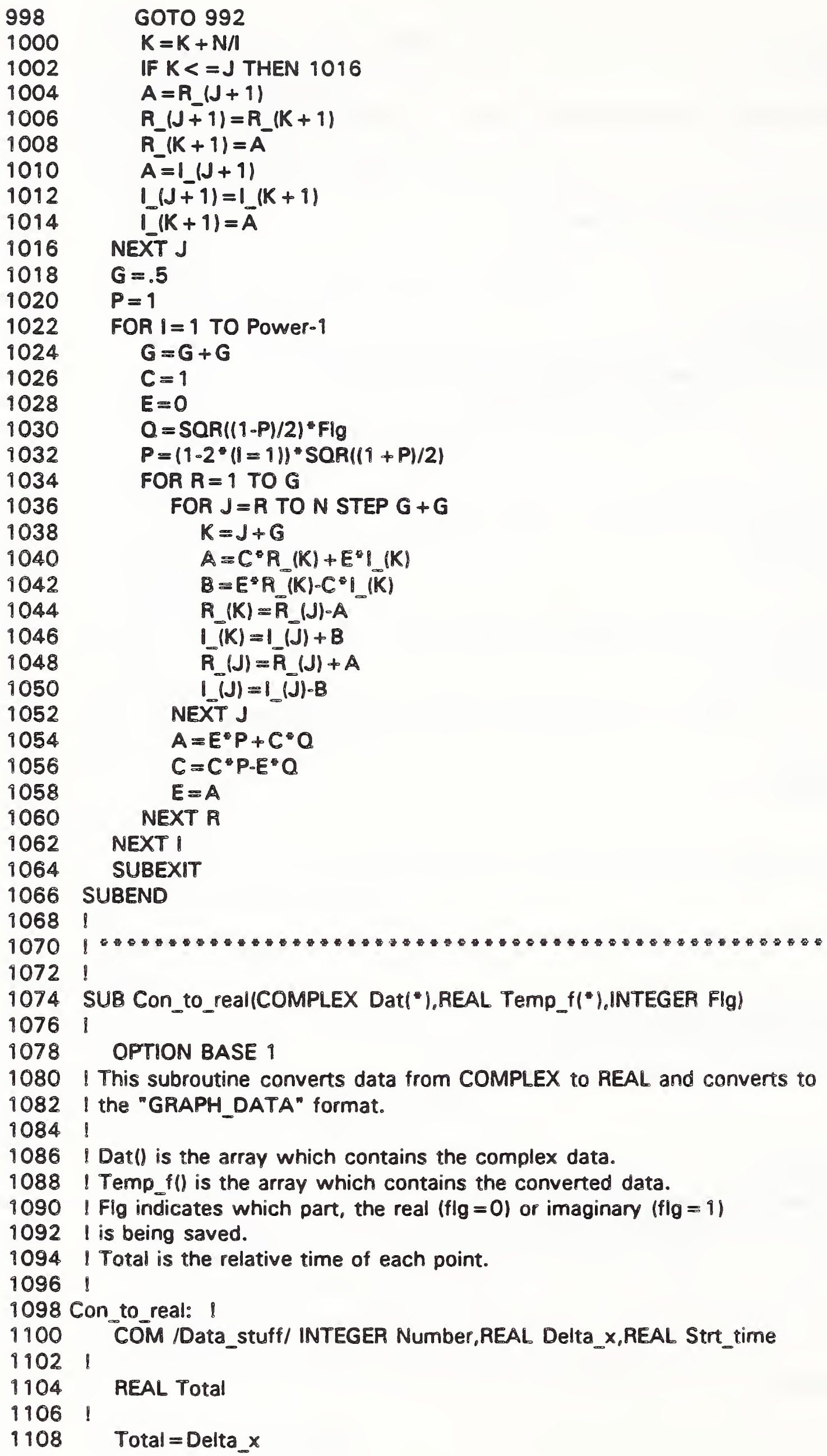


1110

1112

1114

1116

1118

1120

1122

1124

1126

1128

1130

1132

1134

1136

1138

1140

1142

1144

1146

1148

1150

1152 I complex format. The first position of the data array contains

FOR $I=1$ TO Number

IF NOT FIg THEN

Temp_f $(1,2)=$ REAL(Dat $(1))$

$$
\text { ELSE }
$$

Temp_f $(1,2)=$ !MAG (Dat(l))

END IF

Temp_f $f(1,1)=$ Total

Total $=$ Total + Delta_ $x$

IF Bug2 THEN

PRINT Temp_f $f(1)$, ,Temp_f(l,2)

PRINT I

END IF

NEXT I

SUBEXIT

SUBEND

1

1

SUB Get_data(COMPLEX File("),REAL Total,INTEGER Flg)

1154 I the number of data points in the real part and the imaginary part

1156 I contains the point spacing (delta $t$ ).

1158 I FLG indicates a real $(\mathrm{flg}=0)$ data file, $(\mathrm{flg}=1)$ indicates a

1160 ! complex data file.

1162 I All files are assumed to be $x, y$ pairs by the routine load_disk_data.

1164 ! As such, it is necessary to convert every file to type COMPLEX.

$1166 !$

1168

1170

1172

1174

$1976 \quad$ I

\section{OPTION BASE 1}

COM /nterrupts/ INTEGER !ntr_prty

COM /Files/ Diskdrive $\$[20$ ],Filename $\$[14$ ],Ms_path $\$[500]$

1178 Get_data: !

1180

1182

1184

1186

1188

1190 Read again:

1192

1194

1196

1198

1200

1202

1204

1206

1208

1210

1212

1214

1216

1218

COM /Data_stuff/ INTEGER Number,REAL Delta_x,REAL Strt_time

I

REAL Temp(4096,2)

INTEGER Real_or_cmplx

DIM Dataid\$[40]

Total $=0$

1220

Diskdrive $\$=\infty \%$

Filename $\$=$ *

DISP "Enter the name of the measured waveform when prompted."

WAIT .8

INPUT "Is this data real $=0$ or complex $=1 ", \mathrm{Flg}$

IF (Flg $<>0$ ) AND (Flg $<>1$ ) THEN

DISP "IMPROPER INPUT, please try again."

WAIT 1.0

GOTO Read_again

END IF

Real_or_cmplx $=$ Flg

Intr_prty = Local_prty +3

CALLL Load_disk_data(Temp("),Number,Dataid\$,Flg)

Intr_prty = Local_prty-3

IF FIg THEN 
1272 Record_data: \&

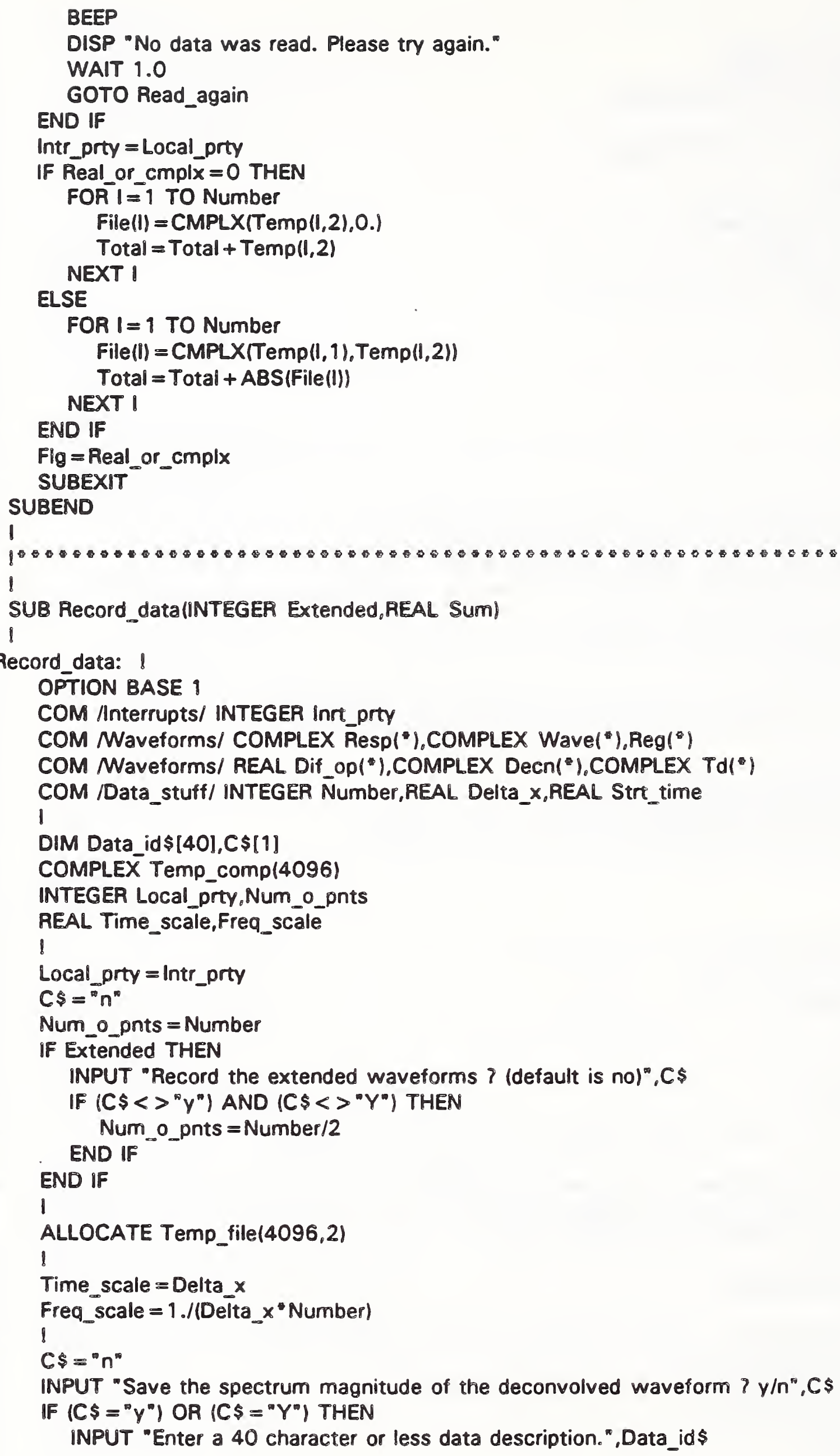


Intr_prty = Local_prty +3

MAT Temp_comp $=$ Decn REDIM. Temp_comp(4096)

Temp $=$ Number

Delta_x $=$ Freq_scale

FOR $\bar{l}=2$ TO Num_o_pnts

IF ABS(Temp_comp (II) $<>0$ THEN

Temp_ $r=\overline{2}$ *ABS $(1 . E+12$ *Temp_comp(I))

Temp_r $=20^{*}$ LGT(Temp_r) I 1E1 $\overline{2}$ IS THE CONVERSION

Last_non_zero $=$ Temp_r I TO MICROVOLTS/MHz

ELSE

Temp_r $=$ Last_non_zero

END IF

Temp_file $(1-1,1)=$ Delta_ $x$

Temp_file $(1-1,2)=$ Temp_p

NEXT I

Delta_ $x=$ Freq_scale + Delta_ $x$

CALL Data_to_disk_r(Temp_file(*),Num_o_pnts-1,Num_o_pnts-1,Data_id\$) Intr_prty = Local_prty

REDIM Temp_file(Number, 2) END IF

1

$C \$=" n "$

1 INPUT "Save the FFT of the response the waveform ? y/n",C\$

IF $(C \$=" Y ")$ OR $(C \$=$ "Y") THEN

INPUT "Enter a 40 character or less data description." Data_id\$

Intr_prty = Local_prty +3

Delta_$x=$ Freq_scale

MAT Temp_comp $=(1 . / \text { Number })^{*}$ Resp

CALL Con_to_real(Temp_comp("),Temp_file("),0)

IF Extended THEN

REDIM Temp_file(Num_o_pnts,2)

END IF

CALL Data_to_disk_r(Temp_file("),Num_o_pnts,Num_o_pnts,Data_id \$) Intr_prty = Local_prity

REDIM Temp_file(Number, 2)

END IF

1

$C \$=" n "$

INPUT "Save the real part of the deconvolution result ? $y / n$ ",C $\$$

IF (C $\$=" Y ")$ OR (C $\$=$ "Y") THEN

INPUT "Enter a 40 character or less data description." Data_id\$

Intr_prty = Local_prty +3

Delta_ $x=$ Time_scale

CALL Con_to_real(Td(*),Temp_file $(*), 0)$

IF Extended THEN

REDIM Temp_file(Num_o_pnts,2)

END IF

CALL Data_to_disk_r(Temp_file("),Num_o_pnts,Num_o_pnts,Data_id\$)

Intr_prty = Local_prty

REDIM Temp_file(Number,2)

END IF

1

$C \$=" n^{n}$

INPUT "Save the imaginary part of the deconvolution result $? y / n^{m}, C \$$ 
IF (C\$ = "Y") OR (C\$ = "Y") THEN INPUT "Enter a 40 character or less data description." Data id $\$$ Intr_prty $=$ Local_prty +3 CALLL Con_to_real(Td/),Temp_file $(*), 1)$ IF Extended THEN REDIM Temp_file(Num_o_pnts,2) END IF CALL Data_to_disk_r(Temp_file("),Num_o_pnts,Num_o_pnts,Data_id $\$$ ) Intr_prty $=$ Local_prty

\section{END IF} REDIM Temp_file(Number,2)

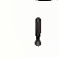


REAL Dtime

ISelect the disk drive where the data exists

1570

1572

1574

1576

1578 Sele

1580

1582

1584

1586

1588

1590

1592

1594

1596

1598

1600

1602

1604

1606

1608

1610

1612

1614

1616

1618

1620

1622

1624

1626

1628

1630

1632

1634

1636 !

IF Overflow $<>0$ THEN Overflow $=0$

Hold_size $=0$

Dtime $=0$.

Allocated $=0$

Selectdrive:

IF Diskdrive \$ = "NO DISK" THEN Diskdrive\$ =" "

IF LEN(Diskdrive $\$$ ) $>0$ THEN GOTO Choosefilename

GRAPHICS OFF

OUTPUT 2 USING "\#,K";"K"

CALL Select_disk

IF Diskdrive $\$=$ "NO DISK" THEN GOTO Mistakelineset

Choosefilename: 1

Tempfile $\$=$ Filename $\$$

IF LEN(Filename $\$$ ) >0 THEN GOTO Bring_in_data

AC $\$=$ "CAT"

CALL Enterfilename(AC $\$$ )

IF LEN(Filename $\$$ ) $=0$ OR POS(Filename $\$, " *$ ") $>1$ THEN

IF POS(Filename $\$, " * \mid>1$ THEN I set mask $\$$

Mask $\$=$ Filename $\$[1, P O S$ (Filename $\$, " *)-1]$

Filename $\$=n$

$$
\text { ELSE }
$$

END IF

Ftype $\$=$ "BDAT " I examine BDAT files only

Fls_cnt $=1$ I select one file

Intr_prty = Local_prty + 1

CALL_ File_menu(Mask $\left.\$, F t y p e \$, F I s \$(*), F i s \_c n t, 0,0\right)$

intr_prty = Local_prty

Filename $\$=$ FIs $\$(1)$

IF LEN(Filename $\$$ ) $=0$ THEN $!$ aborted

Filename $\$=$ Tempfile $\$$

GOTO Mistakelineset

END IF

END IF

1638 Bring_in_data: I

1640

1642

1644

1646

1648

1650

1652

1654

1656

1658

1660

1662

1664

1666

1668

IFind this file on the disk.

1

ON ERROR GOTO Cant_findfile

ASSIGN @Datapath TO Filename $\$ \&$ Diskdrive $\$$

OFF ERROR

Dtime $=$ TIMEDATE

DISP " LOADING disk file: ";Filename\$;" ... ";

ON ERROR GOTO Bad_file

ENTER @Datapath;Status\$

OFF ERROR

ON ERROR GOTO Cant_findfile

SELECT Status\$

CASE "Y" I All graphics/data parameters exist.REN 100,2

DISP " Complete graph." 
ENTER @Datapath;Time_orgn\$,Date_orgn\$

ENTER @Datapath;Time_chng\$,Date_chng\$ ENTER @Datapath;Description\$ ENTER @Datapath;Labels $\$(")$, Lbl_count,Lbl_addr(") ENTER @Datapath;Curve_id $\$("), \bar{S}_{\text {Yymbol } \$(*)}$ ENTER @Datapath; Roster("),Curvecount ENTER @Datapath;Graphtype $\$$, Margins $\$(*)$ ENTER @Datapath; $X_{-}$cross_y,Y_cross_X ENTER @Datapath;Xgrid_tick $\$, \bar{X}$ major,Xminor ENTER @Datapath;Ygrid_tick\$,Ymajor,Yminor ENTER @Datapath;Xmin_graph,Xmax_graph ENTER @Datapath;Ymin_graph,Ymax_graph

CASE " $N$ " I Only data parameters exist. DISP " RAW data."

CASE ELSE

Bad_file: DISP CHR\$(12)

DISP "Data file is not recognized, entry aborted.": DISP " ...continue."

BEEP

PAUSE

OFF ERROR

GOTO Mistakelineset END SELECT

I

ENTER @Datapath:Data_id\$

IF FIg THEN

ENTER @Datapath;Delta_x ENTER @Datapath;Datacount

ELSE Hold_size $=$ Datacount

ENTER @Datapath;Datacount ENTER @Datapath;Hold_size

END IF

IF NOT Allocated THEN

If Datacount $>=1$ AND Hold size $>=1$ THEN ALLOCATE Holding_file(Hoild_size, 2)

ELSE ALLOCATE Holding_file $(1,2)$

\section{END IF}

Allocated $=1$

END IF

ENTER @Datapath;Holding_file(")

ASSIGN @Datapath TO *

OFF ERROR

IF NOT FIg THEN

Delta_ $x=$ Holding_file $(2,1)$-Holding_file $(1,9)$

Strt_time $=$ Holding_file $(1,1)$

END IF

IF Datacount $=0$ THEN Mistakeline

!

Copy data from Holding_file(") to Basket_file(") !

MAT Basket_file $=10.1$

IF Datacount $>$ Basketsize THEN IReceiving file too small.

Allocated $=0$

DEALLOCATE Holding_file( $(")$ 
DISP " DATA FILE overflow, new data discarded. ";

1790

1792

1794

1796

1798

1800

1802

BEEP

PAUSE

IF Status $\$=$ " $Y$ " THEN

Curvecount $=0$

MAT Roster $=(0)$

\section{END IF}

Overflow $=$ Hold size

GOTO Mistakelineset

1804 Copydatafile: $\quad 1$

1806 FOR $R=1$ TO Datacount

1808

1810

1812

1814

1816

1818

1820

1822 Mistakelineset: Datacount $=0$

1824

1826 Mistakeline:OFF KEY

1828 IF Allocated THEN DEALLOCATE Holding_file(")

1830 LOOP

1832 EXIT IF TIMEDATE-Dtime $>1.8$

1834

1836

1838

1840

1842

1844

1846

1848 Cant_findfile: IError in searching for the file.

1850

1852

1854

1856

1858

1860

1862

1864

1866

1868

1870

1872

1874

1876

1878

1880

1882

1884

1886

1888

END LOOP

DISP CHR\$(12)

OUTPUT 2 USING "\#,K";"K"

SUBEXIT

1

| IIIIIIIIIIIIIIIIIIIIIIIIIIIIIIIIIIIIIIIIIIIIIIIIIIIIIII

1

BEEP 500,.6

SELECT ERRN

CASE 56

DISP "That file does not exist on this disk ":

CASE $72,73,76,82$

DISP Diskdrive $\$ ; "$ has failed or is not available ":

CASE ELSE

DISP ERRM\$:

END SELECT

DISP "...CONTINUE to try again."

PAUSE

Filename $\$=$ "

Diskdrive $\$="$ "

GOTO Selectdrive

I

SUBEND

1

1

SUB Select disk

1890 Select_disk: T Original: 13 Nov 1984

1892

I Revision: 02 Dec 1987 
COM /Files/ Diskdrive $\$[20], F i l e n a m e \$[14], M s \_p a t h \$[500]$

COM /Interrupts/ INTEGER Intr_prty

COM /Sys_msi/ Msi_id\$

COM ISYs/ Sys_ids

INTEGER Local_prty,Dd,Pt,Choose(?)

DIM Disc \$(30)[60],Title\$[40],Displ\$[60]

Local_prty = Intr_prty

OFF KEY

I

I Define the disk drives available for this system, reserve the

I first characters for the drive address and the characters after

I the - for a description of the drive.

1

I Example:

( $\operatorname{Disc} \$(1)={ }^{\circ}, 700,0,0$ HP 9133 H HARD disk volume $0 .{ }^{\circ}$

!

1

Displ\$ = "SELECT DISK DRIVE ... Abort will cancel."

Title $\$=$ "Available disk drives for this system. "

$P r=1$ I allow only one select

1

IF Diskdrive $\$[1,1]<>$ : $^{*}$ THEN Diskdrive $\$={ }^{\infty}$

IF MSi_id $\$[1,1]<>":$ THEN MSi_id $\$$ =SYSTEM ("MSI")

IF Msi_id $\$[1,1]<>{ }^{\circ}:$ THEN I Must be HFS subdirectory

Ms_path\$ $=$ Msi_id\$[1,POS(Msi_id\$,":")-1] I strip off subdirs

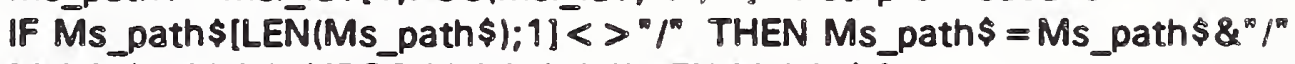

Msi_id $\$$ Msi_id \$[POS(Msi_id\$,":" ),LEN(Msi_id\$)]

END IF

Diskdrive $\$=$ TRIM $\$$ (Diskdrive $\$$ )

Msi_id $\$=$ TRIM $\$$ (Msi_id\$)

IF LEN(Diskdrive $\$$ ) $>0$ AND LEN(MSi id $\$$ ) >0 THEN

Disc $\$(1)=$ Diskdrive $\$$ \&RPT $\$(*, 17$-LEN(Diskdrive $\$ 1)$

Disc $\$(1)=$ Disc $\$(1) \&^{n}$ - Last selected disk drive."

$\mathrm{Dd}=1$

If Diskdrive $\$>$ Msi_id $\$$ THEN

Disc $\$(2)=$ Msi_id \$\&RPT $\$\left({ }^{\circ}\right.$ ".17-LEN(Msi_id\$1)

Disc $\$(2)=$ Disc $\$(2) \& "$. Start-up mass storage unit specifier."

$D d=D d+1$

ELSE

ELSE

$\operatorname{Disc} \$(1)=\operatorname{Disc} \$(1) \& "$ Start-up MSUS."

END IF

IF LEN(MSi id\$) >0 THEN

Disc $\$(1)=$ Msi_id \$\&RPT $\$\left(1^{\circ} * 17\right.$-LEN(Msi_id $\left.\$ 1\right)$

Disc $\$(1)=$ Disc $\$(1) \& "$. Start-up mass storage unit specifier."

$\mathrm{Dd}=1$

ELSE

$\mathrm{Dd}=0$

END IF

END IF

2004 
2078 Enterfilename: ! Original: 13 Nov 1984

1 Disc $\$(D d+1)=":, 702,0$ - HP 9122 dual microfloppy left drive" Disc $\$(D d+2)=":, 702,1 \quad$ - HP 9122 dual microfloppy right drive" Disc $\$(D d+3)=":, 703,0 \quad-$ HP 9125 single 5.25 floppy drive" Disc $\$(D d+4)=":, 1400 \quad-$ HP 9133H hard disk volume 1" I $D d=D d+4 \quad I$ add the number of drive specifiers above I

IF Sys_id $\$[1,4]<>$ "S300" THEN

Disc $\$(D d+1)=": 4,1$ - LEFT internal series $200 "$

Disc $\$(D d+2)=":, 4,0$ - RIGHT internal series 200"

END IF

!

CALL Menu scroll(Displ\$,Title\$,Disc\$("),Dd,Pt,Choose("))

IF $P t=0$ THEN

Diskdrive\$ = "NO DISK"

ELSE

Dd $=$ POS (Disc $\$($ Choose (Pt)), " -" )-1 I find -

IF Dd>5 THEN I valid msus

ELSE

Diskdrive $\$=$ TRIM $\$$ (Disc $\$(C h o o s e(P t))[$ ( , Dd $]$ )

DISP " ERROR in reading MSUS from string, - chr not found."

BEEP

CALL Pause key_on

Diskdrive $\$=$ "NO DISK"

END IF

SUBEXIT

SUBEND

1

\section{OPTION BASE 1}

COM /Files/ Diskdrive\$[20],Filename\$[14],Ms_path\$[500]

COM /nterrupts/ INTEGER Intr_prty

INTEGER I,Ascii_num,Maskflag,Namelength

DIM Test\$[256],Hfs_temp\$[161]

Namelength $=10$

IF LEN(Ms_path\$) >0 THEN OUTPUT KBD USING "K, \#" ;" \#" \&Ms_path\$\&" $H^{m}$ DISP " ENTER HFS directory PATH (no file)";

IF AC $\$<>$ "PATH" THEN

DISP ", ENTER / for HFS ROOT or null for LIF...";

\section{END IF}

LINPUT Hfs_temp\$

Hfs_temp\$ $=$ TRIM\$ (Hfs_temp\$)

IF LEN(Hfs_temp\$)>0 THEN

IF LEN(Hfs_temp\$) $>1$ AND Hfs_temp\$[LEN(Hfs_temp $\$) ; 1]<>" /$ THEN Hfs_temp $\$=H f s_{-} t e m p \$ \& " /$

END IF

IF LEN(Hfs_temp $\$)=1$ THEN Hfs_temp $\$=$ " 
END IF

IF AC $\$=$ "PATH" THEN

Ms_path $\$=H f s \_t e m p \$$

SUBEEXIT

END IF

IF LEN(Filename $\$$ ) $>0$ THEN OUTPUT KBD USING ${ }^{n} K, \#^{n} ;^{\infty} \#^{\infty}$ \&Filename $\$ \&^{n} H^{\infty}$

DISP " ENTER the FILE NAME ... ":

SELECT AC\$

CASE "CAT"

DISP "(ENTER CAT mask " or ENTER null to CAT)":

CASE "ABORT"

DISP "(ENTER null to ABORT) ":

CASE "VALID"

DISP "(must be a VALID namel)";

END SELECT

LINPUT Test\$

Test\$ = TRIM\$(Test\$)

IF LEN(Test $\$$ ) =0 AND Ac $\$=$ "VALID" THEN GOTO Enterfilename

IF LEN(Test $\$)=0$ THEN Abortline

IF LEN(Test\$) > Namelength THEN

BEEP

DISP "ERROR in NAME ENTRY - max "NNamelength:" chars, you have ":

DISP LEN(Test\$1; " "

WAIT 1.8

OUTPUT 2 USING "K, \#";" \#" \&Test\$\&" U $^{\infty}$

GOTO Efn

END IF

IF POS(Test\$, $\left.{ }^{* *}\right)>1$ THEN

Test $\$=$ Test $\$[1$, POS(Test\$, " ")-1]

Maskflag $=1$

ELSE

$$
\text { Maskflag }=0
$$

END IF

FOR $1=9$ TO LEN(Test $\$$ )

Ascii_num $=$ NUM(Test $\$[1])$

SELECT Ascii_num

CASE 65 TO $90,95,97$ TO 122,48 TO 57

IAllowed characters

CASE ELSE

BEEP

DISP "ERROR in NAME ENTRY-ILLEGAL CHARACTERS, TRY AGAIN."

WAIT 1.8

OUTPUT 2 USING "K, \#";"\#"\&Test\$\&"H"

GOTO Efn

\section{END SELECT}

NEXT I

IF Maskflag THEN

ELSE

Filename $\$=$ Test $\mathrm{\&}^{\mathrm{m}}$ "

Filename $\$=$ Test $\$$

END IF

Ms_path $\$=H f s_{\text {_temp }}$

SUBEXIT

2228 Abortline:Filename $\$={ }^{n}$ " 
2242 SUB File_menu(Mask\$,Ftype\$,Fls\$(*),INTEGER Fls_cnt,Dir_on,Prt_on)

2244 File_menu: !

2246 I Original: 29 Jun 1987, G. Koepke

2248 I Revision: 02 Dec 1987, 07:00

DEG

COM /Sys/ Sys_id\$[10]

COM /Files/ Diskdrive $\$[20$, Filename $\$[14], M s$ path $\$[500]$

COM /nterrupts/ INTEGER Intr_prty COM /Bugs/ INTEGER Bug1,Bug2,Bug3,Printer DIM Directory $\$(600)[80], B d \$(600)[71]$ DIM D\$[80],T\$[51],Ids\$[40],Stat\$[1],Test\$[256] INTEGER Bd_cnt,File_cnt,I,C_cnt,CO(1),Format_error,End_search IF FIs_cnt $>0^{-}$THEN ÁLLOCÁTE INTEGER Choose(FIs_cnt) I

I Catalog the disk specified 1 End_search $=0$

REPEAT I Generate path to file and extract file name.

ON ERROR GOTO Cat_errors

DISP " Reading the Directory ..."

IF LEN(Ms_path\$) >0 THEN

MASS STORAGE IS Ms_path\$[1,LEN(Ms_path\$1-1]\&Diskdrive\$

\section{ELSE}

MASS STORAGE IS Diskdrive\$

\section{END IF}

CAT TO Directory \$ $(*$ );NO HEADER,COUNT File_cnt OFF ERROR

!

I set up array of legal file names.

1

Bd_cnt $=0$

MÁT Bd\$ $=(" n)$

FOR $I=1$ TO File_cnt

SELECT Directory \$ (I) $[32,36]$

CASE Ftype $\$$ I Ftype $\$=$ "BDAT" or I Ftype $\$=$ "PROG"

IF LEN(Mask\$) >0 THEN \& Test for mask $\$$

IF Directory \$ (1) [1, LEN(Mask\$) $=$ Mask $\$$ THEN

Bd_cnt $=$ Bd_cnt +1

$\mathrm{Bd} \overline{\boldsymbol{S}}\left(\mathrm{Bd} \_\mathrm{Cnt}\right)=$ Directory $\$(1)(1 ; 14] \& "$ - "\&Ftype $\$$

ELSE END IF

$B d_{-} c n t=B d \_c n t+1$ END IF

$B d \vec{s}\left(B d \_c n t\right)^{-}=$Directory $\$(1)[1 ; 14] \& "$ - "\&Ftype $\$$

CASE "DIR "

Bd_cnt $=B d \_c n t+1$

Bd\$(Bd_cnt $)^{-}=$Directory $\$(I)[1 ; 14] \& "$ - DIR "

2340 
NEXT I

IF LEN(Ms_path $\$$ ) $>0$ AND Bd_cnt $>0$ AND Fls_cnt $>0$ THEN $B d_{-} \mathrm{cnt}=\mathrm{Bd} \_\mathrm{cnt}+1$

$B d \bar{s}\left(B d \_c n t\right)="$ - MOVE back up ONE Directory level."

Bd_cnt $=B d \_c n t+1$

$B d \bar{s}\left(B d \_c n t\right)="$ RETURN to ROOT Directory. ${ }^{\circ}$ END IF

1

I set up file menu

1

$D \$="$ Select "\&VAL\$(Fls_cnt)\&" file name(s) for data entry." $T \$=$ "List of "\&Ftype \& \&"files and DIRs on "\&Diskdrive\$

If LEN(Mask\$) >0 THEN

$T \$=T \$ \& "$ mask $=$ " \&Mask $\$$

END IF

If Bd_ent $>0$ THEN

If Dir_on $>0$ THEN GOSUB Read_data_id

IF Prt on THEN

GOSUB List_directory

End $\operatorname{search}=1$

ELSE

C_ent $=$ Fls_cnt

DISP CHRS (12)

IF Fis_ent $>0$ THEN

CALL Menu_scroll(D\$,T\$,Bd\$("),Bd_ent,C_ent,Choose("))

ELSE

CALL Menu_scroll(D\$,T\$,Bd\$("),Bd_cnt,C_cnt,CO(*))

END IF

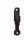

I transfer file names to Fls\$ $(*)$.

l

IF $C_{\text {C }}$ Cnt $=0$ THEN I selection process aborted

End_search $=1$

MATे FIs $\$=(" m)$

ELSE

MAT SORT Choose(")

FOR I $=9$ TO C Cnt

IF Bd\$(Choose (l) ) $[18,22]=$ Ftype $\$$ THEN

Fls $\$(1)=B d \$($ Choose $(1))[1 ; 14]$

End_search $=1$

ELSE $I$ it must be a Directory or message.

SELECT Bd\$(Choose (l)) $[18,22]$

CASE "up ON" I move up one directory

LOOP

Ms_path\$ = Ms_path\$ [9,LEN(Ms_path\$)-1]

EXIT IF LEN(MS_path $\$$ ) $=0$

Test $\$=$ Ms_path $\$$ [LEN(Ms_path \$):1]

EXIT IF Test $\$=" / n$

END LOOP

CASE "ROOT * $!$ jump to root directory

Ms path $\$=$ " *

CASE "DIR " 1 add directory to Ms_paths

Test $\$=$ TRIM \$ (Bd \$ (Choose $(1))(1,14])$

Ms_path $\$=$ Ms_path $\$ \& T e s t \$ \& / "$

CASE ELSE 
2508 Read_data_id: I This routine expects to see Ids $\$$ from END IF

DISP " Reading file contents ... Please stand by. " PRINT TABXY $(1,18)$;" Reading \#";

FOR $\mid=1$ TO Bd_cnt $\mid$ each BDAT file PRINT TABXȲ $(11,18)$;

PRINT USING "3D,4A,3D,2A,\#";I," of "Bd_cnt," . "

Ids $\$=$ "Data not recognized."

IF Bd\$() $[18,22]=$ "BDAT " THEN

ON ERROR GOTO Not_recognized

ASSIGN @lo_path TO Bd\$(I) $[1 ; 14]$

ENTER @Io_path;Stat\$

SELECT Stat\$

\section{CASE " $N$ "}

ENTER @lo_path;lds\$

CASE " $Y$ " Ids $\$=$ "Complete graph in GRAPH_DATA form."

2544 Not_recognized:ASSIGN @lo_path TO 
PRINT TABXY(1, 18);RPT $\$(" \circ, 40)$;

2578 Interpret_1: I This is used to interpret ID strings.

2580 Format_error $=1$

2582 identify this particular format

2584 RETURN

2586

2588

2590

2592 List_directory: 1 This routine will provide a tabular listing of

2594

2596

2598

2600

2602

2604

2606

2608

2610

2612

2614

2616

2618

2620

2622

2624

2626

2628

2630

2632

2634

2636

2638

2640

2642

2644

2646 Printer_kaput:DiSP " Printer not responding ... listing aborted."

2648

2650

2652

2654

2656

1

I IIIIIIIIIIIIIIIIIIIIIIIIIIIIIIIIIIIIIIIIIIIIIII 1

I the directory along with Ids\$ if provided

1

DISP " Listing directory ..."

ON TIMEOUT 7,10 GOTO Printer_kaput

PRINTER IS Printer

PRINT USING "//"

PRINT T\$

IF LEN(Ms_path\$1 >0 THEN PRINT "HFS Path: ":Ms_path

PRINT RPT $\$\left({ }^{\circ}={ }^{\circ}, 80\right)$

PRINT "File name":

IF Dir_on THEN

PRINT * - TYPE ... contents"

ELSE

PRINT * - TYPE"

END IF

PRINT RPT $\$\left({ }^{\circ} \circ{ }^{\circ}, 80\right)$

FOR $\{=1$ TO Bd_cnt

IF Bd\$(1) $[18, \overline{22}]=$ Ftype $\$$ OR Bd\$(1) $[18,22]=$ "DIR " THEN PRINT Bd\$(!)

END IF

NEXT !

PRINT RPT\$ 1 \%

PRINT

PRINTER IS CRT

OFF TIMEOUT 7

RETURN

2658

2660

2662

2664 SUB Menu_scroll(D\$.T\$,Items\$("),INTEGER Item_cnt,To_select,Choose ("))

2666 Menu_scroll:i Original: 22 Jun 1987, Galen Koepke, NBS 723.04

2668

2670

2672

2674

2676

I Revision: 22 Aug 1990, 12:00, Dennis Cameil I

I A general purpose menu utility for scroiling items and I selecting either a fixed number or a random number 1 of items. 
1 for fixed : To_select $>0$

1 for random : To_select $=-1$

1 The items are arranged in screens of 15 items each and

I the user may access screens via softkeys. There may be

I up to 40 screens or 600 items to choose from.

I Maximum sizes: $D \$[80], T \$[51]$, Items $(*)[70]$

2692

2694

2696

2698

2700

2702

I Items $\$\left({ }^{*}\right)$ contains the item descriptions

I Item_cnt is the number of items in Items $\$(*)$

I Choose $(")$ is dimensioned to the number of required choices

1 and will be filled with the item numbers chosen.

I To_select is the number of required choices.

$$
1
$$

\section{OPTION BASE 1}

PRINTER IS CRT

DEG

GOSUB Def_variables

GOSUB Define_screens

GOSUB Make_selections

IF Null_file THEN I reset to zero

Item_cnt $=0$

Items $\$(1)="$ "

END IF

\section{SUBEXIT}

1

| |IIIIIIIIIIIIIIIIIIIIIIIIIIIIIIIIIIIIIIIIIIIIIIIII

COM /nterrupts/ INTEGER Intr_prty

COM /Bugs/ INTEGER Bug1,Bug2,Bug3,Printer

COM /Sys/ Sys_id\$[10] INTEGER Local_prty,Skips,Knobcount,Pointeractive,KO,Null_file INTEGER Exit_flag,Temp,Random_select,Indx DIM Marker\$[8],Test\$[256] 1

I initialize parameters 1 Local_prty $=$ Intr_prty IF Local_prty $<1$ THEN Local_prty $=10$ IF LEN(SYS_id $\$$ ) $=0$ THEN SYS_id $\$=$ SYSTEM $\$($ SYSTEM ID")

IF Item_cnt $<1$ THEN

Null_file $=9$

Item_cnt $=1$

To_select $=0$

Items $\$(1)=\cdots$ Empty * *

ELSE

Null_file $=0$

END IF

IF To_select $=-1$ THEN

Random_select $=1 \quad 1$ choose random number of items

To_select $=0 \quad$ I needed for softkeys

2788

END IF

IF To_select >Item_cnt THEN To_select $=$ Item_cnt 
Skips $=0$

Knobcount $=0$

Doneflag $=0$

Marker $\$="==>$ "\&RPT $\$(\operatorname{CHR} \$(8), 4)$

2808 Define_screens:l Set up screens of 15 items each.

2810

2812

2814

2816

2818

2820

2822

2824

2826

2828

2830

2832

2834

2836

2838

2840

2842

2844

2846

2848

2850

2852 Make selections:l MENU setup and use.

$K O=0$

Exit_flag $=0 \quad$ allow ENTER key to exit when selections filled.

_loop: 1

ON KBD,Local_prty GOSUB Process_kbd

ON KNOB .01,Local prty GOSUB Move_pointer

IF Random_select THEN

I set keys for random selection

DISP DS

ON KEY KO LABEL " Select" "Local_prty GOSUB Select_pandom

ON KEY KO + 9 LABEL "Accept" "Local_prty GOTO Exit_line

ELSE ! set key KO for fixed selection

IF Skips < To_select THEN

DISP D\$

IF To select $>1$ THEN

$$
\text { Test } \$=\text { "Select "\&VAL\$(Skips + 1)\&" of "\&VAL\$(To_select) }
$$

ELSE

$$
\text { Test } \$=\text { "Select" }
$$

END IF

2900 
ELSE

DISP " Selection process complete ..."

DISP " Menu for information only ... "

END IF END IF

ON KEY KO LABEL "Accept",Local_prty GOTO Exit_line

END IF

IF Active_screen < Screen_cnt THEN

ON KEYY KO + 1 LABEL " Next Screen",Local_prty GOSUB Next_screen ELSE

2974 Escape_line:Skips $=0$

END IF

IF Active_screen $>1$ THEN ELSE

ON KEYY KO + 2 LABEL " Last Screen",Local_prty GOSUB Last_screen

OFF KEY $\mathrm{KO}+2$

END IF

IF Skips $>0$ OR Random_select THEN

ON KEY KO + 3 LABEL " Reset Select",Local_prty GOSUB Select_reset ELSE

OFF KEY KO +3

END IF

IF To_select $>0$ OR Random_select THEN

ON KEY KO + 4 LABEL " Ábort ",Local_prty GOTO Escape_line

ELSE

OFF KEY KO + 4

END IF

IF Screen_cnt $>2$ THEN ELSE

ON KEY KO + 6 LABEL "Jump to Screen",Local_prty GOSUB Jump_to_scn

2976

2978

2980 Exit line:OFF KEY

2982 MAT SORT Choosel")

2984 OFF KNOB

2986 OFF KBD

2988 OUTPUT KBD;CHR\$(255)\&CHR\$(75);

2990 PRINT CHR\$(128);

2992 I everything cleared, now go back to work.

2994 RETURN

$2996 \quad$ I

2998 I IIIIIIIIIIIIIIIIIIIIIIIIIIIIIIIIIIIIIIIIIIIIIIIIII

3000

3002 Next_screen: !

3004 OFF KBD

3006 OFF KNOB

3008 OFF KEY

3010 IF Active_screen $=$ Screen_cnt THEN RETURN

3012 Active_screen $=$ Active_screen +1 


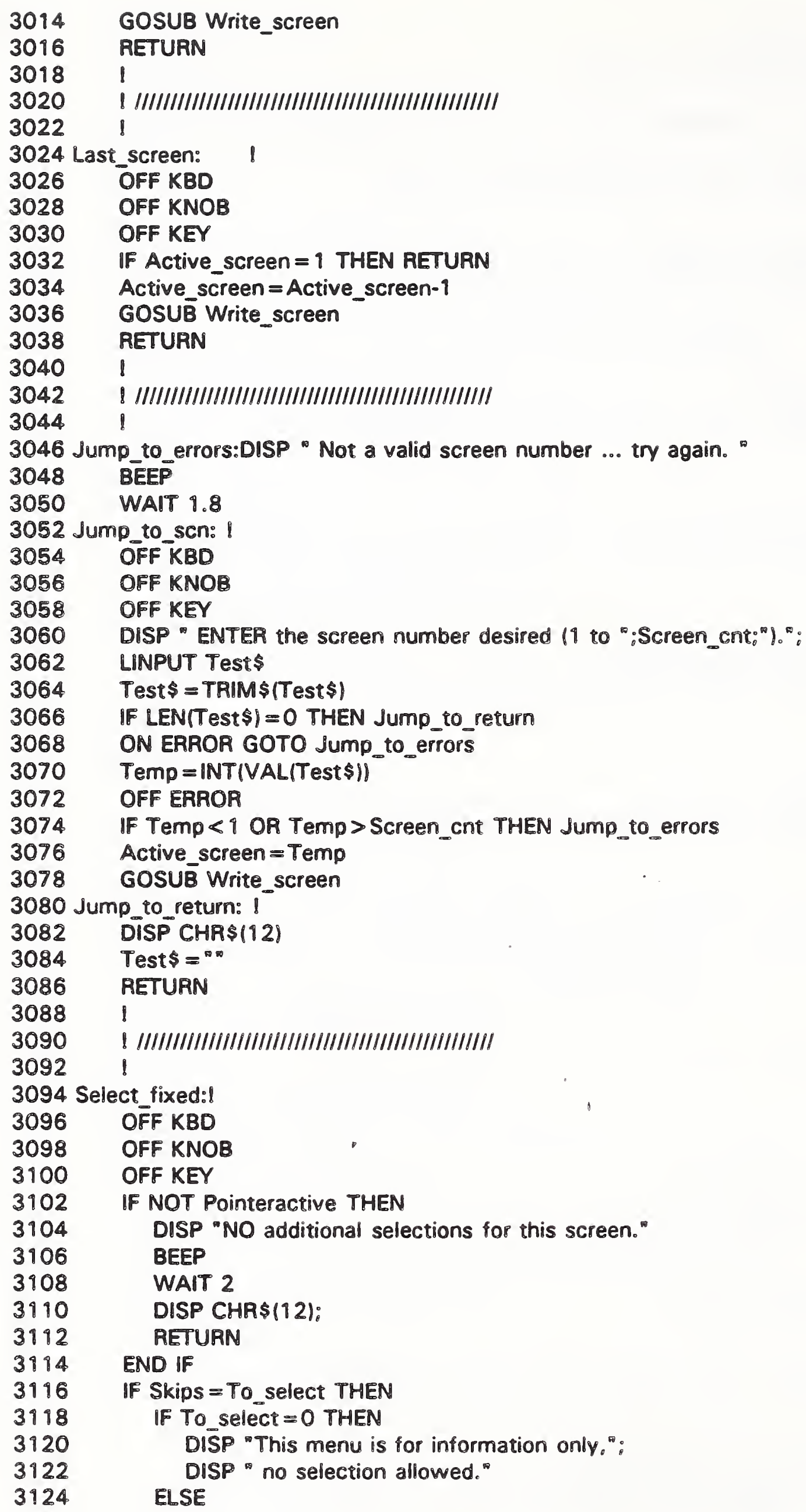




\section{END IF}

DISP "All selections have been filled,":

END IF

BEEP

WAIT 2

DISP CHR\$(12);

RETURN

Skips = Skips + 1

Choose (Skips) $=$ First_item (Active_screen) + Pointer-First_line

PRINT CHR\$(129); I inverse video

PRINT TABXY(10,Pointer);Items\$(Choose(Skips))

PRINT CHR\$(128);

PRINT TABXY(1,Pointer);

SELECT Pointer

CASE First line

GOSUB Point_forward

CASE Last line GOSUB Point_backward

CASE ELSE

I move forward unless it requires wrapping to beginning.

IF Skips-1 >0 THEN I check for selected items.

$I=$ Pointer-First_line

LOOP

$$
\mathrm{K}=0
$$

FOR $J=1$ TO Skips

IF First_item(Active_screen) $+1=$ Choose(J) THEN $K=1$ NEXT $J$

EXIT IF $K=0$

$I=I+1$

$|F|+$ First_line $>$ Last_line THEN $K=-1$

EXIT IF $K=-\overline{1}$

END LOOP

IF $K=0$ THEN

GOSUB Point_forward

ELSE

GOSUB Point_backward

ELSE

GOSUB Point_forward

END IF

END SELECT

RETURN

1

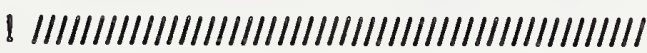

1

OFF KBD

OFF KNOB

OFF KEY

Test $\$=$ "NO"

IF NOT Pointeractive THEN

DISP "NO additional selections for this screen."

BEEP

WAIT 2

DISP CHR\$(12); 
3312 Select_reset: IClear Choose file

3314 OFF KBD

3316 OFF KNOB

3318 OFF KEY

3320 IF Random_select THEN To_select $=0$

3322 Skips $=0$

3324 MAT Choose $=$ (999)

3326

3328

3330

3332

3334

3336 Process_kbd:! Allow use of aprows and enter key in addition to soft.

3338 Test $\$=$ KBD $\$$

3340 IF LEN(Test\$) = 1 AND Tes $\{[1,1]<>$ CHR $\$(32)$ THEN BEEP 80...1 RETURN

3348 IF Test $\$[1,1]=$ CHR $_{(32)}$ THEN GOSUB Point forward 
IF Test $\$[1,1]<>$ CHR\$(255) THEN RETURN

SELECT Test $\$(2,2]$

CASE CHR\$ $\$ 255)$

CASE " $V$ "," $T$ "

I do nothing

3360

3362 GOSUB Point_forward

3364

3366

3368

3370

3372

3374

3376

3378

3380

3382

3384

3386

3388

3390

3392

3394

3396

3398

3400

3402

3404 Point_forward:Knobcount $=5$

3406 ḠOSUB Move_pointer

3408 RETURN

3410 Point backward: Knobcount $=-5$

3412 GOSUB Move_pointer

3414 RETURN

3416 !

3418

3420

3422 Jog_pointer:! Move the selection pointer on the active screen.

3424

3426

3428

3430

3432

3434

3436

3438

3440

3442

3444

3446

3448 Move_pointer:! Control pointer to avoid re-selection of items

3450

3452

3454

3456

3458

3460 I without regard to selected values

IF Knobcount >0 THEN I Move forward

$$
\text { Pointer }=\text { Pointer }+1
$$

ELSE

I Move backward

Pointer $=$ Pointer -1

END IF

IF Pointer < First line THEN Pointer = Last_line

IF Pointer $>$ Last_line THEN Pointer $=$ First_line RETURN

1

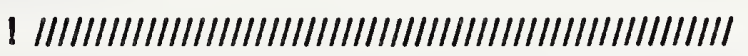

1

IF NOT Pointeractive THEN RETURN I No selections to be made.

Knobcount $=$ Knobcount + KNOBX-KNOBY

IF ABS(Knobcount) $<4$ THEN RETURN

Last_pt $=$ Pointer

GOSUB Jog_pointer

IF Skips $>0$ THEN 
3508

3510

3512

3514

3516 Write screen:I Write the screen pointed to by Active screen

3518

3520

3522

3524

3526

3528

3530

3532

3534

3536

3538

3540

3542

3544

3546

3548

3550

3552

3554

3556

3558

3560

3562

3564

3566

3568

3570

3572

\section{LOOP}

$J=$ Pointer-First_line

FOR $I=1$ TO Skips

IF First_item(Active_screen) $+J=$ Choose(I) THEN $J=999$

NEXT I

If $J=999$ AND Pointer $=$ Last_pt THEN Pointeractive $=0$

EXIT IF Pointeractive $=0$

IF $J=999$ THEN GOSUB Jog_pointer

EXIT IF $J<>999$

END LOOP

END IF

Knobcount $=0$

OUTPUT KBD;CHR\$(255)\&CHR\$(84); I Bring screen home

IF Last_pt $=$ Last_line THEN PRINT CHR\$(132);

PRINT " *:

IF Pointeractive THEN I Pointer active

If Pointer $=$ Last line THEN

PRINT CHR\$(132):

ELSE

PRINT CHR\$(128):

END IF

PRINT TABXY(1,Pointer):Marker \$:CHR\$(128):

END IF

RETURN

l

! IIIIIIIIIIIIIIIIIIIIIIIIIIIIIIIIIIIIIIIIIIIIIII

!

I home and clear screen

OUTPUT KBD;CHR\$ (255)\&CHR\$ (84)\&CHR\$ (255)\&CHR\$ (75):

Knobcount $=$ KNOBX-KNOBY I Clear knob and keyboard

Knobcount $=0$

Test $\$=$ KBD $\$$

Test $\$={ }^{m \text { m }}$

l

PRINT TABXY(1, First_line-1):CHR\$(132);" Item \# Screen \#":

PRINT USING "\#,2D,4A,2D,3A";Active_screen。" of ":Screen_cnt; $\left.{ }^{\circ}\right|^{\text {" }}$

PRINT T\$:RPT \$ ${ }^{\circ} "$ "59-LEN(T\$ $):$

PRINT TABXY (80, First line-1):" 1 " CHR\$ 1128$)$ :

$J=0$

REPEAT

IF $\mathrm{J}=$ Last item(Active screen)-First_item(Active_screen) THEN PRINT CHR\$(132):

PRINT TABXY $\left(1\right.$, First_line $+J 1$ :RPT $\$\left({ }^{\circ}{ }^{\circ}, 80\right)$

ELSE

PRINT CHR\$(128):

END IF

PRINT TABXY(5,First_line + J);

PRINT USING " $3 D, A, \#^{\infty}{ }^{\circ}$ First item(Active screen) $+J_{0}{ }^{\circ} 1^{\circ}$

IF Random_select THEN

FOR $I=\overline{9}$ TO TO_select

IF First_item(Äctive_screen) $+J=$ Choose(l) THEN

PRINT CHR\$ (129):

END IF

NEXT I

ELSE 
3674 SUB Data_to_disk_r(REAL File("),INTEGER Filesize,Datacount,Data_id\$)

3676 Data_to_disk_r: I Original: 13 Nov 1984

If Skips $>0$ THEN I make this line inverse video FOR $I=1$ TO Skips

IF First_item(Active_screen) $+\mathrm{J}=$ Choose(I) THEN END IF PRINTT CHR\$(12) $\overline{9})$;

END IF

END IF

PRINT TABXY $(10$,First_line $+\mathrm{J})$; Items $\$$ (First_item(Active_screen) $+\mathrm{J}$ )

PRINT TABXY $\left(80\right.$, First_line + J); $\left.\right|^{n}$;

$\mathrm{J}=\mathrm{J}+1$

UNTIL J $>=$ (Last_item(Active_screen)-First_item(Active_screen) +1 )

Last_line $=$ Last_item(Active_screen)-First_item(Active_screen)

Last_line $=$ Last_line + First_line

1

I set marker to first non-selected item.

!

Pointeractive $=0$

IF To_select $>0$ OR Random_select THEN Pointeractive $=1$

IF Skips $>0$ AND Pointeractive $=1$ THEN I find first non-selected item $J=0$

LOOP

Pointer $=$ First_line $+\mathrm{J}$

FOR I $=1$ TO Skips

IF First_item(Active_screen) $+J=C$ Choose(I) THEN Pointer $=0$ NEXT I

EXIT IF Pointer $<>0$

$\mathrm{J}=\mathrm{J}+1$

IF First_line $+J>$ Last_line THEN

Pointeractive $=0$

END IF

Pointer $=$ First_line

EXIT IF Pointer $<>0$

END LOOP

ELSE

Pointer $=$ First_line

END IF

IF Pointeractive THEN

IF Pointer = Last_line THEN PRINT CHR\$(132);

ELSE

PRINT CHR\$(128):

END IF

PRINT TABXY(1,Pointer);Marker\$;CHR\$(128);

\section{END IF}

RETURN

SUBEND

1

I Revision: 06 Aug 1987

I This routine will SAVE data files on the disk in RAW data format.

I Special features:

I If the Diskdrives and/or the Filename\$ are null this routine 
$3686 \quad$ will prompt the operator for information. However, if they

$3688 \quad 1$ are not null it is assumed that the program is supplying the

$3690 \quad \&$ correct information.

3692

3694

3696

3698

3700

3702

3704

3706

3708

3710

3712

3714

3716

3718 Selectdrive: 1

3720 IF Diskdrive $\$$ "NO DISK" THEN Diskdrive $\$=$ "

3722 IF LEN(Diskdrive\$)>0 THEN GOTO Choosefilename

3724 GRAPHICS OFF

3726 OUTPUT 2 USING "\#,K";"K"

3728 CALL Select disk

3730 If Diskdrive $\$$ " NO DISK" THEN GOTO Mistakeline

3732 Choosefilename: 1

3734 IF LEN(Filename\$) >0 THEN GOTO Send_to_disk

$3736 \quad$ AC $\$={ }^{~}$ ABORT

3738 CALL Enterfilename(AC\$)

3740 IF LEN(Filename $\$$ ) $=0$ THEN GOTO Mistakeline

3742 Send_to_disk: I Create file and save information.

3744 ON ERROR GOTO Cant_savedata

3746 Diskspace $=$ INT $(($ Filesize $16.01 / 256)+2$

3748 CREATE BDAT Filename\$\&Diskdrive\$,Diskspace, 256

3750 Dtime $=$ TIMEDATE

3752 DISP " SAVING data in file "Filename $\$$ " on "Diskdrive\$

3754 Status $\$={ }^{\text {"N }}$ "

3756 ASSIGN @Datapath TO Filename\$\&Diskdrive\$

3758 OUTPUT @Datapath;Status\$

3760 OUTPUT @Datapath;Data_ids 140 chrs description of data

3762 OUTPUT @Datapath;Datacount Inumber of xy points

3764 OUTPUT @Datapath;Filesize Isize of arpay

3766 OUTPUT @Datapath; File(")

3768 ASSIGN @Datapath TO.

3770 ÓFF ERROR

$3772 \quad 1$

3774 Mistakeline:OFF KEY

3776 LOOP

3778 EXIT IF TIMEDATE-Dtime $>1.8$

3780 END LOOP

3782 DISP CHR\$(12)

3784 OUTPUT 2 USING "\#, $K^{*}: " K^{*}$

3786 SUBEXIT

$3788 \quad 1$

$3790 \quad$ I IIIIIIIIIIIIIIIIIIIIIIIIIIIIIIIIIIIIIIIIIIIIIIIIIIIIII

$3792 \quad 1$

3794 Cant_savedata: I

3796 BEEP 500.6 


\section{SELECT ERRN}

CASE $72,73,76,78,81,82,90,93$

3852 Pause_key_on: I Make sure that CONTINUE key exists.

3854

3856

3858

3860

4022 Errortrap: ! Original: 13 Nov 1984

4024

4026

4028

4030

4032

4034

DISP "....CONTINUE to try again."

\section{PAUSE}

Diskdrive $\$="$ "

CASE 84,85

DISP " This disk is not initialized ";

DISP "....CONTINUE to try again."

PAUSE

Diskdrive $\$=$ " "

CASE 55,64

DISP " This disk is full, insert new floppy and/or";

DISP " select new drive ...CONTINUE "

PAUSE

Diskdrive $\$="$ "

CASE ELSE

CALL Errortrap

IF LEN(Filename\$) >0 THEN GOTO Send_to_disk

END SELECT

GOTO Selectdrive

!

SUBEND

TOriginal: 02 Dec 1987

I Revision: 02 Dec 1987

OPTION BASE 1

COM /Sys/ Sys_id\$[10]

IF Sys_id\$ $[1,4]=$ "S300" THEN ! reset to $\$ 300$ system keys

CONTTROL KBD, 15;0

CONTROL CRT, 12;2

END IF

PAUSE

IF Sys_id $\$[1,4]=$ "S300" THEN I set to $\$ 200$ compatible keys

OUTPUT KBD USING "K, \#";" SCRATCH KEYX"

CONTROL KBD, 15;1

END IF

CONTROL CRT, 12;0

SUBEXIT

SUBEND

1

1

SUB Errortrap

I Revision: 02 Dec 1987

1 Trap most errors here

OPTION BASE 1

COM /Files/ Diskdrive $\$[20]$, Filename $\$[14], M s$ path $\$[500]$

DIM File\$[20], Test\$[256], What\$[20],Ac\$[5]

BEEP 400,6 
4036

4038

4040

4042

4044

4046

4048

4050

4052

4054

4056

4058

4060

4062

4064

4066

4068

4070

4072

4074

4076

4078

4080

4082

4084

4086

4088

4090

4092

4094

4096

4098

4100

4102

4104

4106

4108

4910

4912

4914

4116

4118

4120

4922

4124

4926

4128

4130

\section{SELECT ERRN}

CASE 54

DISP "DUPLICATE FILE NAME: ";Filename\$;

DISP "...PURGE old one? (Y/N)";

LINPUT What\$

What $\$=$ TRIM $\$$ (What $\$$ )

SELECT What $\$ 1,1]$

CASE " $Y ", " Y$ "

PURGE Ms_path\$\&Filename $\$$ \&Diskdrive $\$$

CASE ELSE

$A C \$=$ "VALID"

CALL Enterfilename(AC\$)

END SELECT

CASE 52,53

DISP "Improper FILE NAME - ENTER NEW FILE NAME";

OUTPUT 2 USING "\#,K,K";"\#";Filename\$

LINPUT Filename\$

Filename $\$=$ TRIM $\$$ (Filename $\$$ )

CASE 56

DISP "FILE: ":Filename $\$_{;}^{\prime \prime}$ is not on this disk, please insert";

DISP " correct disk"

CALl Pause_key_on

CASE 64

DISP "This disk is full, PLEASE insert clean disk"

CALL Pause_key_on

CASE 56

DISP "DATA INPUT disk must be in drivell ":

DISP "...CONTINUE when ready."

CALL Pause_key_on

CASE $72,73,7 \overline{6}$

DISP Diskdrive\$;

DISP ${ }^{*}$ is not available, type correct"

DISP " unit specifier (ie. ':,707,0').";

OUTPUT 2 USING " $K$, \#";Diskdrive\$

LINPUT Diskdrive\$

CASE 80

DISP "CHECK DISK drive door!"

CALL Pause_key_on

CASE ELSE

DISP ERRM \$; "CONTINUE' when fixed"

CALL Pause_key_on

END SELECT

DISP CHR\$ (12)

SUBEXIT

SUBEND

1 1 


\section{B. 3 FIXACO}

102

104

106

108

110

112

114

116

118

120

122

124

126 Date_line: !

128

130

132

134

136

138

140

142

144

146

148

150

152

154

156

158

160

162

164

166

168

170

172 Fixacq: !

174 !

176

178

180

182

184

186

188

190

192

194

196

198

200

202

204

206

208

!

1

END

!

1

!

RAD

$!$

!

1
I RE-STORE "FIXACQ:, 1400"

COM /Sys/ Sys_ids[10]

COM /Sys_msi/Msi_id\$[20]

OUTPUT KBD USING "K,\#";"SCRATCH KEYE" IERASE SOFT KEYS

CONTROL KBD, 15;0! sets the color of the soft keys

CONTROL KBD, $2 ; 1$

! Program by S.M. Chesnut. The Nationa! Institute of Standards

I and Technology. Based on a program by W. Gans and R. Stafford.

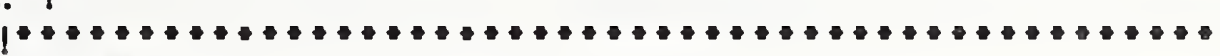

I Last Modified May 17,1991 by S.M.C

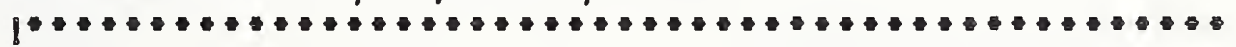

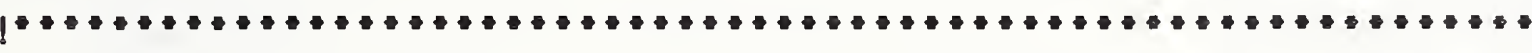

intr $p r t y=9$

CALL Fixacq

MASS STORAGE IS ":,1400"! Resets the mass storage device to

! the hard drive. This number may be

I changed to suit.

OUTPUT KBD USING "K, \#";"LOAD KEYE"! restore the typing aid keys

PRINT TABXY $(1,5) ; "$ END of program. So long."

SUB Fixacq

OPTION BASE 1

! This program reads in the following data:

1 The device under test (DUT) waveform, Wave,

I the voltage calibration data ,Vcal,

$!$ and the time calibration data, Tca!.

! The DUT data is then "fixed" using the calibration data.

COM /Interrupts/ INTEGER Intr_prty

COM /Sys_msi/ Msi_id\$[20]

COM /Sys/ Sys_ids[10]

COM /Bugs/ INTEGER Bug1,Bug2,Bug3,Printer

COM /Files/ Diskdrive $\$[20]$, Filename $\$[14], M s$ _path $\$[500]$

SYs_id = SYSTEM\$("SYSTEM ID") 
290 DIM Vcal $(100,2)$, Tcal $(100,2)$, Wave $(4096,2)$

238 Dut: DISP "Input DUT waveform file name when prompted"

266 Vcaldata: DISP "Input voltage calibration file name when prompted ${ }^{*}$

INTEGER Datacount,Fig,Vfilesize,Tfilesize,Local_prty,Stp_flg

DIM Data_id $\$[40], C h \_v c a l \$[1], C h \_t c a l \$[1]$

$$
\text { Datacount }=32767
$$

Vfilesize $=4096$

Tfilesize $=4096$

$$
\mathrm{Flg}=0
$$

Ch_vcal $=$ ต

Ch_tcal $\$={ }^{\circ}$ "

Local_prty = intr prty

Intr_prty = Intr_prty +2

Filename $\$={ }^{n-m}$

Diskdrive $\$={ }^{\circ \text { w }}$

\section{WAIT 1.0}

CALL Load_disk data(Wave ("),Datacount,Data_id\$,Flg)

IF NOT FIg THEN

\section{BEEP}

DISP "NO FILE IN MEMORY, TRY AGAIN."

WAIT 9.5

GOSUB Reset_filestuff

GOTO Dut

END IF

GOSUB Reset filestuff

REDIM Wave(D̄atacount, 2)

INPUT "Is voltage calibration desired? $y / n^{*}{ }_{0} C_{\text {_ }} v$ cal $\$$

IF Ch_vcal $\${ }^{\circ} \gamma^{*}$ OR Ch_vcal $\$={ }^{\text {" }} y^{\text {" }}$ THEN

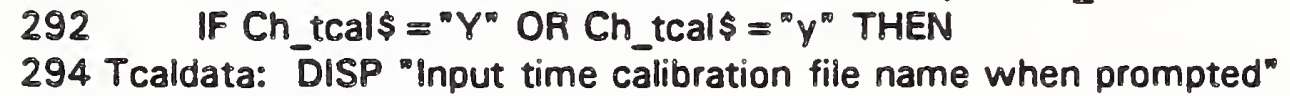
WAIT 1.0

CALL Load_disk_data(Vcal( $\%$,Vfilesize,Data_id\$,Flg)

IF NOT FIg THEN

BEEP

DISP "NO FILE IN MEMORY. TRY AGAIN."

WAIT 9.5

GOSUB Reset_filestuff

END IF GOTO Vcaldata

END IF

GOSUB Reset_filestuff

INPUT "Is time scale calibration desired? y/n",Ch_tcal\$ WAIT 1.0

CALL Load_disk_data(Tcal("),Tfilesize,Data_id\$.Flg)

IF NOT Flg THEN

BEEP

DISP "NO FILE IN MEMORY, TRY AGAIN."

WAIT 1.5

GOSUB Reset_filestuff

GOTO Tcaldata

END IF

GOSUB Reset_filestuff

ALLOCATE True_i(Datacount)

END IF

CALL Real_pnt_time(Tcal("),True_^("),Tfilesize,Datacount)

ALLOCATE Cal_wv(Datacount,2)

Intr_prty = Local_prty 
IF Ch_vcal\$ $=$ " $y$ " OR Ch_vcal\$ $=" Y$ " THEN

360 Reset filestu

END IF

IF Ch_tcal\$ = "Y" OR Ch_tcal\$ = " $y "$ THEN

\section{ELSE} CÁLL Fix_time(Wave ("),True_t("),Cal_wv("),Datacount,Stp_flg)

MAT Cal_wv(*,1) = Wave( $(, 1)$

MAT Cal_wv(*,2) $=$ Wave $(*, 2)$

END IF

IF NOT Stp_flg THEN

INPUT "Enter a 40 character description of the data." Data_id\$

Intr_prty = Intr_prty + 2

CALL Data_to_disk_r(1,Datacount,Cal_wv("),Data_id \$)

Local_prty $=$ Intr_prty

\section{END IF}

SUBEXIT

362

364

366

368

370

372

374

376

378

380

382

386

388

390

392

394

396

398

400

402

404

406

408

410

412

414

416

418

420

422

424

426

428

430

432

434

436

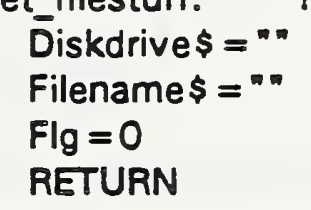




\section{True} NEXT J

I Now the points after the final zero crossing interval.

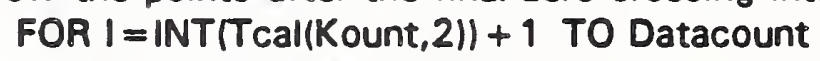


566 SUB Fix_time(REAL Wv(*),True_t(*),Cal_wv(*),INTEGER Datacount,Stp_fig)

568 I

570 Fix_time: !

572 !

578 i

580 COM TTcal_vars/ REAL Point,Kount,Scope_window,Real_window

582 REAL Dt,Extrp

584 INTEGER Opt

586

588

590

592

594

596 1

Opt $=0$

1

$D t=$ Scope window/Point

1

598

600

602

604

606

608

610

612

614

616

618

620

622

624

626

Cal_wv $(1,1)=W v(1,1)$

Cal_wv $(1,2)=W v(1,2)$

Cnt $=2$

$J=1$

WHILE Cnt $<=$ Datacount

Temp $=D t *(C n t-1)$

WHILE (True_t(J) < Temp) AND (J<Datacount) $J=J+1$

END WHILE

IF J>Point THEN GOTO Stp_loop

Cal_wv $(\mathrm{Cnt}, 1)=\mathrm{Wv}(\mathrm{Cnt}, 1)$

Cal_wv $(C n t, 2)=W v(J-1,2)+(W v(J, 2)-W v(J-1,2))=($ Temp-True_t $(J-1)) /\left(\right.$ True_t $\left.(J)-T r u e \_t(J-1)\right)$ IF Bug 1 THEN

PRINT Cnt;" BEFORE ";Wv(Cnt,2):

PRINT" AFTER ";Cal_wv(Cnt,2)

\section{END IF}

$\mathrm{Cnt}=\mathrm{Cnt}+1$

628

630

632 Stp_loop: I

634 PRINT Cnt-1:"POINTS CORRECTED"

636 If Real_window $<$ (Scope_window-Dt) THEN

638 CLEAR SCREEN

640 PRINT "The true time window is less than the scope time window."

642 Select_opt:

644 PRINT "Your options are to:"

646 PRINT "(0) Abort"

648 PRINT "(1) Don't correct the time base"

650

652

654

656

658

660

PRINT " $(2)^{\circ}$ Correct and output fewer data points"

PRINT "(3) Extrapolate using the last data point value"

PRINT "(4) Extrapolate using a value input from the keyboard "

PRINT " $(5)$ Extrapolate using the mean value of the last $5 \%$ of the data"

PRINT "(6) Extrapolate using the mean slope of the last $5 \%$ of the data" INPUT "Your choice ?",Opt 
762 SUB File_menu(Mask\$,Ftype\$,FIs $\$(\%)$,INTEGER Fis_cnt,Dir_on,Prt_on)

764 File_menu: !

766 I Original: 29 Jun 1987, G. Koepke

768 I Revision: 02 Dec 1987, 07:00

770 OPTION BASE 1

772 DEG

Five_percent $=$ Cnt $-1-$ INT $\left(.05^{*}\right.$ Datacount $)$

SELECT Opt

CASE 0

$$
\text { Stp_flg }=1
$$

FOR $I=1$ TO Datacount

Cal_wv $(1,1)=W v(1,9)$

Cal_wv $(1,2)=W v(l, 2)$

SE 2

CASE 3

FOR $\mathrm{I}=$ Cnt TO Datacount

Cal_wv $(J, 1)=$ Cal_wv $(C n t-1,1)$

NEXT I

CASE 4

INPUT "Value for extrapolation?" Extrp

FOR $I=$ Cnt TO Datacount

Cal_wv $(1,2)=$ Extpp

NEXT

CASE 5

Extrp $=0$.

FOR $1=$ Five percent TO Cnt 1

NEXT I

Extpp $=$ Extpp/(1-9-Five_percent)

FOR $I=$ Cnt TO Datacount

Cal $w v(1,2)=$ Extpp

Cal_wv $\left.\left(l_{\theta}, 9\right)=W v\left(l_{0}\right\}\right)$

\section{CASE 6}

Extrp $=\left(C a l \_w v(C n t-1,2)-C a ! \_w v(\right.$ Five_percent,2) $) /(C$ Cnt-Five_percent-1)

FOR $1=$ Cnt TO Datacount

Cal $w v(1,2)=$ Extrp

Cal wov $(1,1)=W v(1,1)$

NEXT I

CASE ELSE

DISP "That is not one of your choices. Try again."

END SELECT

END IF

Bug $1=0$

SCREEN

SUBEND 
COM /Sys/ Sys_id\$[10]

COM /Files/ Diskdrive $\$[20$ ],Filename $\$[14], M s$ path $\$[500]$

COM /Interrupts/ INTEGER Intr_prty

I set up array of legal file names.

1

Bd_cnt $=0$

MATT Bd\$ $=(n n)$

FOR I $=1$ TO File_cnt

SELECT Directory $\$(1)[32,36]$

CASE Ftype $\$$ I Ftype $\$=$ "BDAT " or I Ftype $\$=$ "PROG "

IF LEN(Mask \$ ) >0 THEN | Test for mask \$

IF Directory $\$(1)[1$, LEN(Mask $\$)]=$ Mask $\$$ THEN Bd_cnt $=$ Bd_cnt +1

END IF $B d \bar{s}(B d$ Cnt $)=$ Directory $\$(I)[1 ; 14] \& "$ 。 \&Ftype $\$$

ELSE

Bd_cnt $=$ Bd_cnt +1

Bd $\overline{\$}\left(B d \_c n t\right)=$ Directory $\$(1)[1 ; 14] \& "$ - \&Ftype $\$$

END IF

CASE "DIR " I plus all "DIR " listings

Bd_cnt $=$ Bd_cnt +1

Bd $\$(8 d$ cnt $)=$ Directory $\$(I)[1 ; 14] \&^{\infty}$ - DIR *

\section{CASE ELSE}

END SELECT

NEXT I

IF LEN(Ms_path $\$$ ) $>0$ AND Bd_cnt $>0$ AND FIs_ent $>0$ THEN

Bd_cnt $=$ Bd_ent +1

$B d \bar{s}\left(B d_{-} c n t\right)="$ - MOVE back up ONE Directory level."

Bd_cnt $=$ Bd_cnt +1

END IF

Bd $\bar{s}\left(B d \_c n t\right)="$ RETURN to ROOT Directory."

1

I set up file menu

1

$D \$=$ " Select " \&VAL\$(FIs_cnt)\&" file name(s) for data entry." 
$T \$=$ "List of "\&Ftype \& \& files and DIRs on " \&Diskdrive $\$$

IF LEN(Mask\$)>0 THEN

$$
T \$=T \$ \& \text { mask }=\text { " \&Mask } \$
$$

END IF

IF Bd_ent $>0$ THEN

IF Dir_on $>0$ THEN GOSUB Read_data_id

IF Prt on THEN

GOSUB List_directory

ELSE

$$
\text { End_search }=1
$$

\section{C_cnt $=$ Fis_cnt} DISP CHRS (12)

If Fis_cnt $>0$ THEN

CALL Menu_scroll(D\$,T\$,Bd\$("),Bd_cnt,C_cnt,Choosel")l ELSE

CALL Menu_scroll(D\$,T\$,Bd\$(*),Bd_cnt,C_ent,CO(*))

\section{END IF}

$\ell$

\& transfer file names to FIs $\$(")$.

!

IF C_cnt $=0$ THEN I selection process aborted

End_search $=1$

MAT FIS $\$=1 \%$

ELSE

MAT SORT Choose(")

$F O R I=1$ TO C_cnt

IF Bd\$(Choose (II) $[18,22]=$ Ftype $\$$ THEN

Fls $\$(1)=$ Bd $\$$ (Choose $(1))[1 ; 14]$

End_search $=1$

ELSE $I$ it must be a Directory or message.

SELECT Bd\$(Choose(I)) [18,22]

CASE "UP ON" I move up one directory LOOP

Ms_path $\$=$ Ms_path $\$$ [1,LEN(Ms_path \$ $1-1]$

EXIT IF LEN(Ms_path\$) $=0$

Test $\$=M s \_$path $\$$ [LEN(Ms_path $\$$ ):1]

EXIT IF Test $\$=" / n$

END LOOP

CASE "ROOT " $!$ jump to root directory Ms path $\$=$ m

CASE "DIR $\uparrow$ I add directory to Ms_path\$ Test $\$=\operatorname{TRIM} \$(B d \$(C h o o s e(I))[1,94])$ Ms_path $\$=$ Ms_path $\$ \& T e s t \$ \&{ }^{n}$

CASE ELSE DISP "ERROR in directory jump" PAUSE

END SELECT

$I=C_{-} \mathrm{cnt}$

NEXT I

END IF END IF

\section{ELSE}

DISP " This directory contains no ";Ftype $\$ ;$ files ..."

WAIT 2.5

End_search $=1$ 


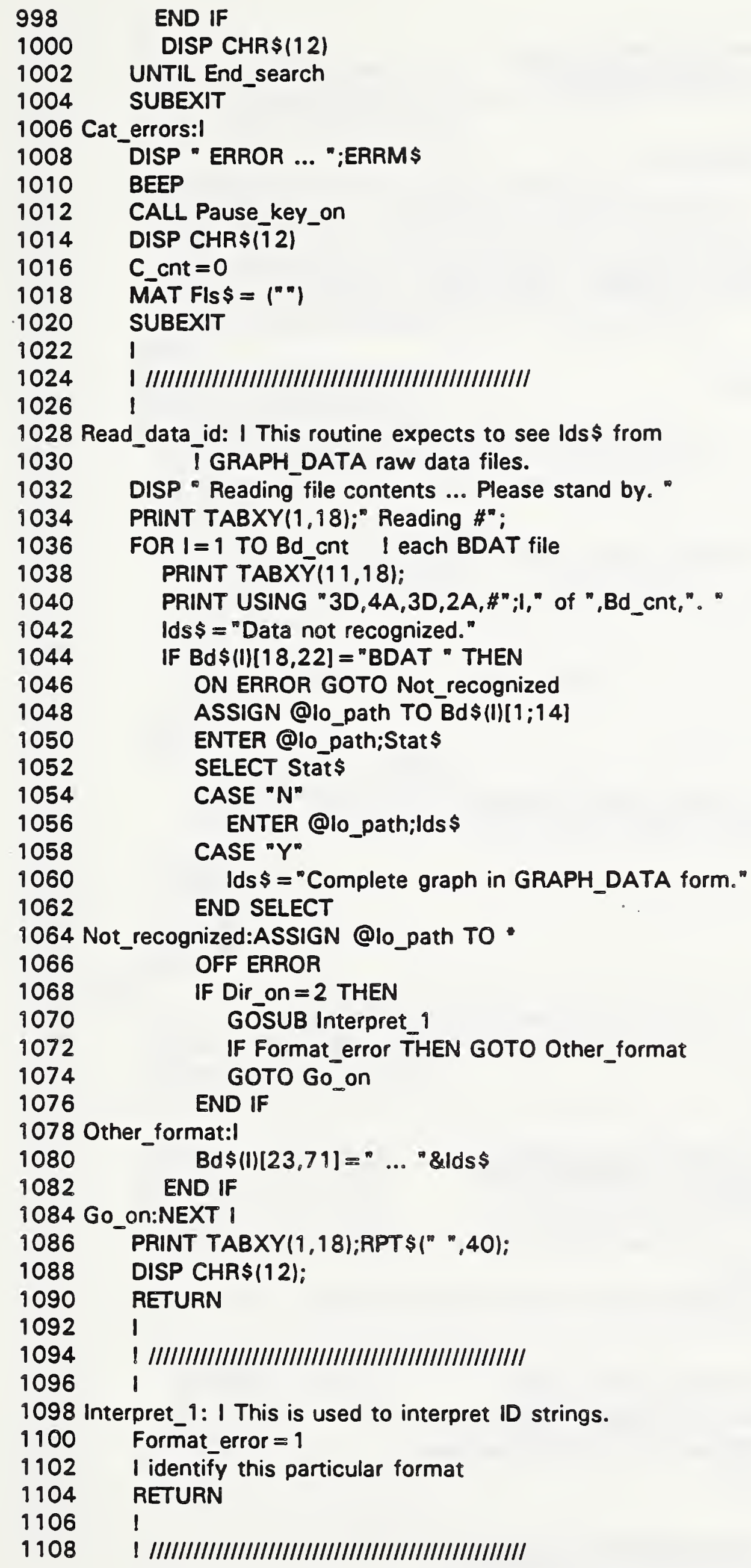


1112 List_directory: 8 This routine will provide a tabular listing of

1166 Printer kaput:DISP "Printer not responding ... listing aborted. "

1168

1970

1972

1174

1176

1178

1180

1982 !

1184 SUB Select_disk

1986 Select_disk: I Original: 13 Nov 1984

1888

1990

1192

1194

1196

1198

1200

1202

1204

1206

1208

1210

1212

1214

1216

1218 I Revision: 02 Dec 1987

OPTION BASE 1

COM /Files/ Diskdrive $\$\left[201\right.$,Filename $\$[14], M s \_p a t h \$[500]$

COM /interpupts/ INTEGER Intr_prty

COM /Sys_msi/ Msi_id\$

COM /Sys/ Sys_ids

INTEGER Local_prty,Dd,Pt,Choose(1)

DIM Disc\$(30)[60],Title $\$[40], D i s p \mid \$[60]$

Local_prty = Intr_prty

OFF KEY !

I Define the disk drives available for this system, reserve the I first characters for the drive address and the characters after I the - for a description of the drive.

| Disc $\$ 19\}={ }^{\circledR}: 700,0,0$ HP 9133 H HARD disk volume $0 .{ }^{\circ}$ 
Displ\$ = " SELECT DISK DRIVE ... Abort will cancel. "

Title $\$=$ "Available disk drives for this system. "

$P_{t}=1 \quad$ I allow only one select I

IF Diskdrive $\$[1,1]<>$ ":" THEN Diskdrive $\$=$ "

IF Msi_id $\$[1,1]<>": "$ THEN Msi_id \$ =SYSTEM $\$(" M S \mid ")$

IF Msi_id\$ $[1,1]<>": "$ THEN I Must be HFS subdirectory

Ms path\$ = Msi id $\$[1, P O S(M s i$ id $\$, ": ")-1]$ I strip off subdirs

IF $\bar{M} s$ path $\$$ (LEN $\left.\left(M s \_p a t h \$\right) ; 1\right]<>" / "$ THEN Ms_path $\$=M s \_p a t h \$ \& " / "$

Msi_id\$ = Msi_id\$[POS(Msi_id\$,":"),LEN(Msi_id\$)

\section{END IF}

Diskdrive $\$=$ TRIM $\$$ (Diskdrive $\$$ )

Msi_id $\$=$ TRIM $\$$ (Msi_id\$)

IF LEN(Diskdrive $\$$ ) $>\overline{0}$ AND LEN(Msi id\$) $>0$ THEN

Disc $\$(1)=$ Diskdrive $\$$ \&RPT $\$(", \overline{17}$-LEN(Diskdrive $\$$ )

$\operatorname{Disc} \$(1)=$ Disc $\$(1) \& "$ - Last selected disk drive."

$\mathrm{Dd}=1$

IF Diskdrive $\$<>$ Msi_id $\$$ THEN

Disc $\$(2)=$ Msi_id\$\&RPT $\$("$ " 17 -LEN(Msi_id $\$ \|$

$\operatorname{Disc} \$(2)=\operatorname{Disc} \$(2) \& "$ - Start-up mass storage unit specifier.

$$
D d=D d+1
$$

ELSE

$$
\operatorname{Disc} \$(1)=\operatorname{Disc} \$(1) \& " \text { Start-up MSUS." }
$$

END IF

ELSE

IF LEN(Msi_id\$) $>0$ THEN

Disc\$(1) $=$ Msi_id\$\&RPT\$(" ",17-LEN(Msi_id\$)

Disc $\$(1)=\operatorname{Disc} \$(1) \& "$ - Start-up mass storage unit specifier."

$$
\mathrm{Dd}=1
$$

ELSE

$$
D d=0
$$

END IF

END IF

1 Customize system drives here

I Follow format with - after unit specifier, description is I optional but recommended. 
1360 Diskselected:OFF KEY

1362

1364

1366

1368

1370

1372

$\$ 376$

1378

1380

1382

1384

1386

1388

1390

1392

1394

1396

1398

1400

1402

1404

9406

9408

9410

1412

1414

1416

1418

1420

1422

1424

1426

1428 Efn:

1430

1432

1434

1436

1438

1440

$\uparrow 442$

9444

ELSE

ELSE

BEEP

END IF

END IF

SUBEXIT

SUBEND

I

1

OPTION BASE 1

Namelength $=10$

END IF

END IF

END IF

SUBEXIT

END IF

SELECT AC\$

CASE "CAT"

CASE "ABORT"

CASE "VALID"
IF $P t=0$ THEN
Diskdrive $\$=$ "NO DISK"

Dd $=$ POS(Disc $\$($ Choose (Pt))," $-"$ ) - ! find -

IF Dd>5 THEN $\mid$ valid msus

Diskdrive $\$=\operatorname{TRIM} \$$ (Disc $\$($ Choose $(P t))[1, D d])$

DISP " ERROR in reading MSUS from string, - che not found."

CALL Pause_key_on

Diskdrive $\$=$ "NO DISK"

SUB Enterfilename(AG\$)

terfilename: I Original: 13 Nov 1984

COM /Files/ Diskdrive \$[20],Filename $\$[14$ ],Ms_path $\$[500$ ]

COM /Interrupts/ INTEGER Intr_prty

INTEGER I,Ascii_num,Maskflag,Namelength

DIM Test\$[256],Hfs_temp\$[161]

IF LEN(Ms_path\$) $>0$ THEN OUTPUT KBD USING ${ }^{\circ} K, \#^{n} ;^{n} \#^{\infty}$ \&Ms_path $\$ \&^{n} H^{n}$

DISP " ENTER HFS directory PATH (no file)":

IF AC\$<> "PATH" THEN

DISP *. ENTER / fOr HFS ROOT or null for LIF...":

LINPUT HfS_temp\$

Hfs temp $\$=$ TRIM $\$($ His temp $\$$ )

IF LEN(Hfs_temp\$) $>0$ THEN

IF LEN(Hfs_temp\$) $>1$ AND His_temp\$[LEN(Hfs_temp\$):1]<>" THEN Hfs_temp $\$=H f s_{-}$temp $\$ \&^{\prime \prime} /{ }^{m}$

IF LEN(Hfs_temp $\$$ ) $=1$ THEN Hfs_temp $\$=$ क

Namelength $=14$

IF AC $\$=$ "PATH" THEN

Ms_path $\$=H f s_{-}$temp $\$$

IF LEN(Filename $\$ \mid>0$ THEN OUTPUT KBD USING ${ }^{n} K_{0} \#^{m} ;{ }^{m} \# "$ \#filename $\$ \&^{n} H^{n}$

DISP " ENTER the FILE NAME ...":

DISP " (ENTER CAT mask or ENTER null to CAT)":

DISP "(ENTER null to ABORT)":

DISP "(must be a VALID name!)": 


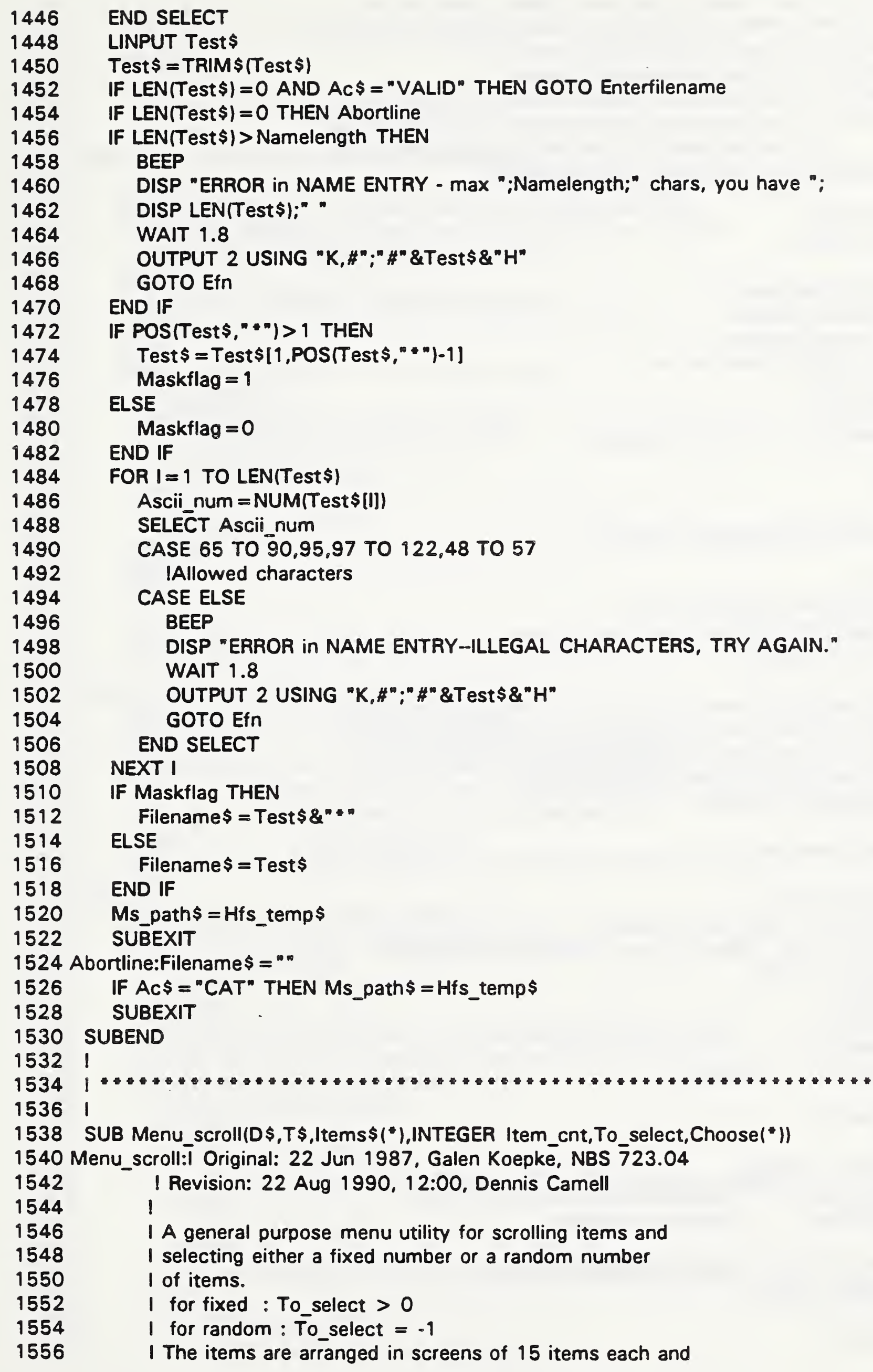


I the user may access screens via softkeys. There may be

I up to 40 screens or 600 items to choose from.

! Maximum sizes: D\$[80], T\$[51], Items (")[70]

1606 Def_variables:!

END IF

I Item_cnt is the number of items in Items $\$(")$

I Choose (") is dimensioned to the number of required choices

1 and will be filled with the item numbers chosen.

1 To_select is the number of required choices. 1

\section{OPTION BASE 1}

PRINTER IS CRT

DEG

GOSUB Def_variables

GOSUB Define_screens

GOSUB Make_selections

IF Null_file THEN I reset to zero

Item_cnt $=0$

Items $\$(1)=$ *

To_select $=0 \quad$ I no valid selections

\section{SUBEXIT}

!

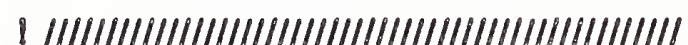

1

COM /interrupts/ INTEGER Intr_prty

COM /Bugs/ INTEGER Bug 1,Bug2,Bug3,Printer

COM /SYs/ SYs_id\$110] 1

INTEGER Screen_cnt,Items_per_scn,First_item(40),Last_item(40) INTEGER I,J,K,First_line,Last_line,Active_screen, Pointer,Last_pt INTEGER Local_prty,Skips,Knobcount,Pointeractive,KO,Null_file INTEGER Exit_flag,Temp,Random_select,Indx DIM Marker\$[8],Test\$[256] 1 I initialize parameters 1 Local_prty $=$ Intr IF Local_prty $<1$ THEN Local_prty $=10$

IF LEN(SYS_id $\$)=0$ THEN SYS_id $\$=$ SYSTEM $\left({ }^{\circ}\right.$ SYSTEM ID $\left.{ }^{*}\right)$

IF Item_cnt $<1$ THEN

Null_file $=9$

Item_ent $=1$

To_select $=0$ Items $\$(1)=\cdots$ Empty * *

ELSE

Null_file $=0$
END IF

IF To select $=-1$ THEN Random_select $=9 \quad !$ choose random number of items To_select $=0 \quad 1$ needed for softkeys

\section{END IF}

IF To_select $>$ Item_cnt THEN To_select $=I t e m_{-}$cnt

MAT Choose $=$ (9999)

Skips $=0$

Knobcount $=0$ 
Doneflag $=0$

1672

Marker $\$="==>$ "\&RPT $\$(\operatorname{CHR} \$(8), 4)$

1674

1676

1678

1680

RETURN

I

1682

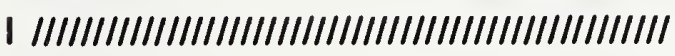

1684

1686

1688

1690

1692

1694

1696

1698

1700

1702

1704

1706

1708

1710

1712

1714

1716

1718

1720

1722

1724

1

1726 Make_selections:! MENU setup and use.

1728

1730

1732

1734

1736

1738

1740

$1742 \mathrm{Key}$

1744

1746

1748

1750

1752

1754

1756

1758

1760

1762

1764

1766

1768

1770

1772

1774

1776

1778

1780

1

Items_per_scn $=15$ I Maximum number of displayable items

IF INT(Item_cnt/ltems_per_scn) =|tem_cnt/items_per_scn THEN

ELSE

Screen_cnt $=$ INT(Item_cnt/Items_per_scn)

END IF

Screen_cnt $=$ INT (Item_cnt/ltems_per_scn) +1

$J=1$

FOR $\mid=1$ TO Screen_cnt $\mid$ set up each screen

First_item $(1)=J$

IF J+ltems_per_scn-1 <item_cnt THEN

Last_item $(I)=J+$ Items_per_scn- 1

$J=J+$ Items_per_scn

ELSE

END IF

NEXT I

RETURN

I

| IIIIIIIIIIIIIIIIIIIIIIIIIIIIIIIIIIIIIIIIIIIIIIIIIII

1

Active_screen $=1 \quad 1$ first screen is active

First_line $=2 \quad$ I first printed line on screen $=2$ or greater.

GOSÜB Write_screen I activate screen at Active_screen

$I$ and set First_line and Last_line for Pointer

KO $=0 \quad$ I Keys start at zero

Exit_flag $=0 \quad I$ allow ENTER key to exit when selections filled.

Key_loop: 1

ON KBD,Local_prty GOSUB Process_kbd

ON KNOB .01,Local_prty GOSUB Möve_pointer

IF Random_select THEN

I set keys for random selection

DISP D\$

ON KEY KO LABEL " Select",Local_prty GOSUB Select_random

ON KEY KO + 9 LABEL "Accept", Local_prty GOTO Exit_line

ELSE ! set key KO for fixed selection

IF Skips<To_select THEN

DISP D\$

IF TO select $>1$ THEN

$$
\text { Test } \$=\text { " Select "\&VAL\$(Skips + 11\&" of "\&VAL\$(To_select) }
$$

ELSE

Test $\$=$ "Select"

END IF ELSE

ON KEY KO LABEL Test\$,Local_prty GOSUB Select_fixed

IF To_select $>0$ THEN

DISP " Selection process complete ..." 
1848

1850

1852

1854

1856

1858

1860

1862

1864

1866

1868

1870

1872

1874

1876 Next

1878

1880

1882

1884

1886

1888

1890

1892

ELSE

DISP " Menu for information only ... "

END IF END IF

ON KEY KO LABEL "Accept",Local_prty GOTO Exit_line

END IF

IF Active_screen < Screen_cnt THEN

ON KEY KO +1 LABEL" Next Screen" "Local prty GOSUB Next screen ELSE

OFF KEY KO +1

\section{END IF}

IF Active_screen $>1$ THEN

ON KEY KO + 2 LABEL " Last Screen", Local_prty GOSUB Last_screen

ELSE

OFF KEY KO +2

END IF

IF Skips >0 OR Random_select THEN

ON KEY KO + 3 LABEL "Reset Select" "Local_prty GOSUB Select_peset

ELSE

OFF KEY KO +3

\section{END IF}

If To select $>0$ OR Random select THEN

ON KEY KO + 4 LABEL "ADbort "Local_prty GOTO Escape line ELSE

OFF KEY KO +4

END IF

IF Screen cnt $>2$ THEN

ON KEY KO +6 LABEL "Jump to Screen" "Local_prty GOSUB Jump_to_scn

\section{ELSE}

OFF KEY KO +6

END IF

IF Exit_flag THEN Exit_line

GOTO Key loop

cape_line:Skips $=0$

MÁT Choose $=(0)$

To select $=0$

xit line:OFF KEY

MAT SORT Choose(

OFF KNOB

OFF KBD

OUTPUT KBD;CHR\$(255)\&CHR\$(75):

PRINT CHR\$1128\%:

I everything cleared, now go back to work.

RETURN

!

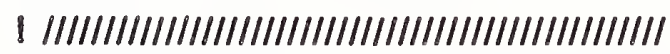

$$
\text { ! }
$$

Xt_screen:

ÖFF KBD

OFF KNOB

OFF KEY

If Active screen $=$ Screen cnt THEN RETURN

Active_screen $=$ Active_screen +1 GOSUB̈ Write_screen RETURN

$$
\text { I }
$$




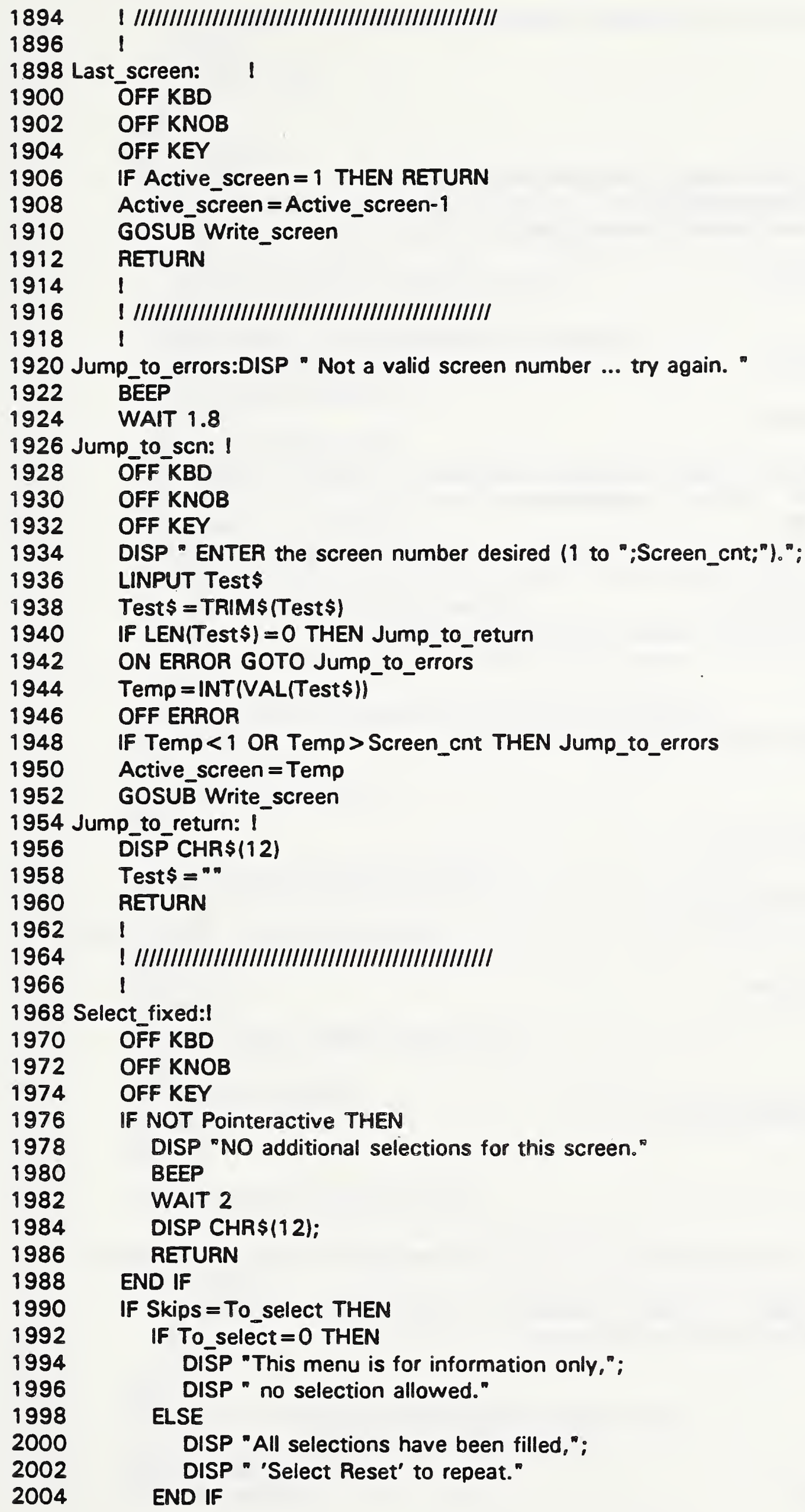


BEEP

2092 Select random:!

2094 OFF KBD

2096 OFF KNOB

2098 OFF KEY

2100 Test $\$=$ "NO"

\section{IF}

$=$ Skips +1

Choose(Skips) = First_item(Active screen) + Pointer-First line PRINT CHR\$(129): I inverse video

PRINT TABXY(10,Pointer);Items \$(Choose(Skips))

PRINT CHR\$(128):

PRINT TABXY(1,Pointer):

\section{SELECT Pointer}

CASE First_line

GOSUB Point_forward

CASE Last_line

GOSUB Point_backward

CASE ELSE

I move forward unless it requires wrapping to beginning.

IF Skips-1 >0 THEN \& check for selected items.

$I=$ Pointer-First_line

LOOP

$K=0$

FOR $\downarrow=1$ TO Skips

IF First_item(Active_screen) $+1=$ Choose(J) THEN $K=1$ NEXT J

EXIT IF $K=0$

$$
1=1+1
$$

IF $\mathbb{I}+$ First line $>$ Last_line THEN $K=-1$

EXIT IF $K=-\overline{1}$

END LOOP

IF $K=0$ THEN

ELSE

GOSUB Point_forward

GOSUB Point_backward

ELSE

GOSUB Point forward

\section{END IF}

END SELECT

\section{RETURN}

IF NOT Pointeractive THEN

DISP "NO additional selections for this screen."

BEEP

WAIT 2

DISP CHR\$ (12):

RETURN

END IF

FOR $I=\{$ TO To_select 
2186 Select_reset: IClear Choose file

2210 Process_kbd:I Allow use of arrows and enter key in addition to soft.

\section{Test $\$=$ KBD $\$$}


2278 Point forward: Knobcount $=5$

2280

2282

2284 Point backward: Knobcount $=.5$

2286 G̈OSUB Move_pointer

2288 RETURN

2290

2292

2294

2296 Jog_pointer:! Move the selection pointer on the active screen. I without regard to selected values IF Knobcount $>0$ THEN ! Move forward

$$
\text { Pointer }=\text { Pointer }+1
$$

ELSE

I Move backward

\section{END IF}

IF Pointer $<$ First line THEN Pointer $=$ Last_line

IF Pointer $>$ Last_line THEN Pointer $=$ First_line RETURN 1

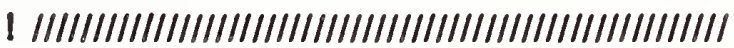

IF NOT Pointeractive THEN RETURN I No selections to be made. Knobcount $=$ Knobcount + KNOBX $\cdot$ KNOBY IF ABS(Knobcount) $<4$ THEN RETURN Last $\mathrm{pt}=$ Pointer GOSUB Jog_pointer IF Skips $>0$ THEN LOOP

$J=$ Pointer-First_line FOR $I=1$ TO Skips 
2390 Write_screen:I Write the screen pointed to by Active_screen

2392 NEXT I IF First_item(Active_screen) $+J=$ Choose(I) THEN $J=999$

IF $J=999$ AND Pointer $=$ Last_pt THEN Pointeractive $=0$

EXIT IF Pointeractive $=0$

IF $J=999$ THEN GOSUB Jog_pointer

EXIT IF J $<>999$

END LOOP

\section{END IF}

Knobcount $=0$

OUTPUT KBD;CHR\$(255)\&CHR\$(84); I Bring screen home

IF Last_pt = Last_line THEN PAINT CHR\$(132);

PRINT " ";

IF Pointeractive THEN I Pointer active

IF Pointer $=$ Last_line THEN

ELSE PRINT CHR\$(132);

PRINT CHR\$(128);

END IF

PRINT TABXY(1,Pointer);Marker\$;CHR\$(128);

\section{END IF}

RETURN

I

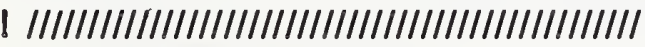
1

thome and clear screen

OUTPUT KBD;CHR\$(255)\&CHR\$(84)\&CHR\$(255)\&CHR\$(75);

Knobcount $=$ KNOBX-KNOBY $\mid$ Clear knob and keyboard

Knobcount $=0$

Test $\$=$ KBD $\$$

Test $\$={ }^{n "}$

!

PRINT TABXY(1,First_line-1);CHR\$(132);" Item \#| Screen \#";

PRINT USING "\#,2D,4A,2D,3A";Active_screen," of ";Screen_cnt;" $\mid$ "

PRINT T\$;RPT \$" ",51-LEN(T\$);

PRINT TABXY(80,First_line-1);" |";CHR\$(128);

$J=0$

REPEAT

IF $J=$ Last_item(Active_screen)-First_item(Active_screen) THEN PRINT CHR\$(132);

PRINT TABXY(1, First_line + J);RPT $\$(", 80)$

ELSE

PRINT CHR\$(128);

END IF

PRINT TABXY (5, First_line + J):

PRINT USING " 3D,A,\#"; First_item(Active_screen) + J," $\mid$ "

IF Random_select THEN FOR I $=\overline{9}$ TO To_select

IF First_item(Active_screen) $+\mathrm{J}=$ Choose(I) THEN PRINT CHR\$(129);

END IF

$$
\text { NEXT I }
$$

ELSE

IF Skips $>0$ THEN I make this line inverse video FOR I $=1$ TO Skips

IF First_item(Active_screen) $+\mathrm{J}=$ Choose(I) THEN 

END IF PRINT CHR\$(129);

NEXT I

END IF

\section{END IF}

PRINT TABXY(10,First_line + J);Items $\$$ (First_item(Active_screen) + J)

PRINT TABXY(80, First_line $+J) ; "$ |":

$J=J+1$

UNTIL $J>=$ (Last_item(Active_screen)-First_item(Active_screen) +1 )

Last_line $=$ Last_item(Active_screen)-First_item(Active_screen)

Last_line $=$ Last_line + First_line

1

I set marker to first non-selected item.

I

Pointeractive $=0$

IF To_select $>0$ OR Random_select THEN Pointeractive $=1$

IF Skips $>0$ AND Pointeractive $=1$ THEN I find first non-selected item $J=0$

LOOP

Pointer $=$ First line $+J$

FOR I $=1$ TO Skips

IF First item(Active screen) $+J=C$ Choose(l) THEN Pointer $=0$ NEXT I

EXIT IF Pointer $<>0$

$J=J+i$

IF First_line $+J>$ Last_line THEN

Pointeractive $=0$

END IF

Pointer $=$ First_line

EXIT IF Pointer $<>0$

END LOOP

ELSE

Pointer $=$ First line

END IF

If Pointeractive THEN

If Pointer $=$ Last_line THEN

PRINT CHR\$ (132):

ELSE

PRINT CHR\$ (128);

END IF

PRINT TABXY(1,Pointer);Marker\$;CHR\$ 1128);

\section{END IF}

RETURN

SUBEND

1

1.

1

2550 Errortrap: I Original: 13 Nov 1984

2552

2554

2556

2558

2560

2562

2564

1 Trap most errors here

OPTION BASE 1

COM /Files/ Diskdrive\$[20],Filename\$[14],Ms_path\$[500]

DIM File $\$[20], T e s t \$[256]$, What $\$[20], A C \$[5]$

BEEP 400,6

SELECT ERRN 
DISP "DUPLICATE FILE NAME: ";Filename\$;

DISP "....PURGE old one? (Y/N)";

LINPUT What\$

What $\$=$ TRIM $\$$ (What $\$$ )

SELECT What $\$[1,1]$

CASE " $Y ", " Y$ "

PURGE Ms_path $\$$ \& Filename $\$$ \&Diskdrive $\$$

\section{CASE ELSE}

AC $\$=$ "VALID"

CALL Enterfilename(AC\$) END SELECT

\section{CASE 52,53}

DISP "Improper FILE NAME - ENTER NEW FILE NAME";

OUTPUT 2 USING "\#,K,K";"\#";Filename\$

LINPUT Filename\$

Filename $\$=$ TRIM $\$$ (Filename $\$$ )

\section{CASE 56}

DISP "FILE: ";Filename $\$ ; "$ is not on this disk, please insert";

DISP " correct disk"

CALL Pause_key_on

\section{CASE 64}

DISP "This disk is full, PLEASE insert clean disk"

CALL Pause_key_on

\section{CASE 56}

DISP "DATA INPUT disk must be in drivell ";

DISP "...CONTINUE when ready."

CALL Pause_key_on

CASE $72,73,7 \overline{6}$

DISP Diskdrive\$;

DISP " is not available, type correct";

DISP " unit specifier (ie. ":,707,0').";

OUTPUT 2 USING "K,\#";Diskdrive\$

LINPUT Diskdrive\$

CASE 80

DISP "CHECK DISK drive doorl"

CALL Pause_key_on

CASE ELSE

DISP ERRM\$;" "CONTINUE' when fixed"

CALL Pause_key_on

END SELECT

DISP CHR\$(12)

SUBEXIT

\section{SUBEND}

1

SUB Data_to_disk_r(INTEGER Curve,Datacount,REAL Basket_file(*),Data_id\$)

Data_to_disk_r: I Original: 13 Nov 1984

I Revision: 02 Dec 1987

IThis routine will SAVE data files on the disk in RAW data format.

OPTION BASE 1

COM /Files/ Diskdrive $\$[20]$,Filename $\$[14], M s$ path $\$[500]$

COM /nterrupts/ INTEGER Intr_prty

INTEGER Local_prty,Diskspace

DIM AC\$[5],Status $\$[1], T e m p f i l e \$[14]$ 
REAL Dtime

Local_prty =Intr_prty

Dtime $=0$.

2692 Selectdrive: $!$

\section{GRAPHICS OFF}

2696 OUTPUT 2 USING "\#,K";"K"

2698 CALL Select_disk

2700 IF Diskdrive $\$$ = "NO DISK" THEN GOTO Mistakeline

2702 Choosefilename: !

$2704 \quad A C \$=" A B O R T$ "

2706 Tempfile $\$=$ Filename $\$$

2708 CALL Enterfilename(Ac\$)

2710 IF LEN(Filename $\$$ ) $=0$ THEN

$2712 \quad$ Filename $\$=$ Tempfile $\$$

2714 GOTO Mistakeline

2716 END IF

2718 Send_to_disk: I Create file and save information.

2720 ON ERROR GOTO Cant_savedata

2722 Diskspace $=$ INT ( Datacount $16.01 / 256)+2$

2724 CREATE BDAT Ms_path\$\&Filename\$\&Diskdrive\$,Diskspace,256

2726 Dtime $=$ TIMEDATE

2728 DISP " SAVING data for CURVE \#"Curve;"。"

2730 Status $\$={ }^{*}$ "

2732 ASSIGN @Datapath TO Ms_path\$\&Filename\$\&Diskdrive\$

2734 OUTPUT @Datapath;Status $\$$

2736 OUTPUT @Datapath;Data_id\$ 140 chrs description if single curve.

2738 OUTPUT @Datapath:Datacount Inumber of xy points

2740 OUTPUT @Datapath;Datacount Isize of array (same as above)

2742 OUTPUT @Datapath;Basket_file(")

2744 ASSIGN @Datapath TO

2746 OFF ERROR

$2748 \quad$ I

2750 Mistakeline:OFF KEY

2752 LOOP

2754 EXIT IF TIMEDATE-Dtime $>1.8$

2756 END LOOP

2758 DISP CHR\$(12)

2760 OUTPUT 2 USING ${ }^{*}{ }^{*}, K^{*} ;{ }^{n} K^{n}$

2762 SUBEXIT

$2764 \quad$ I

2766

2768

2770 Cant savedata: 1

2772 BEEP 500.6

2774 SELECT ERRN

2776

2778

2780

2782

2784

2786

CASE $72,73,76,78,81,82,90,93$

DISP Diskdrive $\$:^{*}$ has failed or is not available ":

DISP "....CONTINUE to try again."

2788

CALL Pause_key_on

Filename $\$=\bar{T}$ emprile $\$$

CASE 84,85

DISP " This disk is not initialized": 
REAL Dtime OFF KEY I

ISelect the disk drive where the data exists 1

IF Overflow $<>0$ THEN Overflow $=0$

Hold_size $=0$

Dtime $=0$.

Allocated $=0$

Selectdrive: !

IF Diskdrive $\$=$ "NO DISK" THEN Diskdrive $\$="$ "

IF LEN(Diskdrive\$)>0 THEN GOTO Choosefilename GRAPHICS OFF

OUTPUT 2 USING "\#,K";"K"

CALL Select_disk

IF Diskdrive $\$=$ "NO DISK" THEN GOTO Mistakelineset

Choosefilename: !

Tempfile $\$=$ Filename $\$$

IF LEN(Filename\$) >0 THEN GOTO Bring_in_data

$A C \$={ }^{\circ}$ CAT"

CALL Enterfilename(AC\$)

IF LEN(Filename $\$$ ) $=0$ OR POS(Filename $\$$ " " I $>1$ THEN

If POS(Filename $\$, "$ i> 1 THEN I set mask $\$$ Mask $\$=$ Filename $\$[1$, POS (Filename $\$, "$ " ") -1$]$

Filename $\$={ }^{*}$

\section{ELSE}

Mask $\$="$ "I no preselection

END IF

Ftype $\$=$ "BDAT * $\mid$ examine BDAT files only

Fls_cnt $=9$ | select one file

Int?_prty = Local_prty + 1

CALL File_menu(Mask\$,Ftype $\left.\$, F i s \$(*), F i s \_c n t, 0,0\right)$

Intr_prty = Local_prty

Filename $\$=$ Fis $\$(1)$

IF LEN(Filename $\$$ ) $=0$ THEN $\mid$ aborted

Filename $\$=$ Tempfile $\$$

GOTO Mistakelineset

END IF

2982 Bring_in_data: 1

2984

2986

2988

2990

2992

2994

2996

2998

3000

3002

3004

3006

3008

3010

3012

IFind this file on the disk.

1

ON ERROR GOTO Cant_findfile

ASSIGN @Datapath TO Filename\$\&Diskdrive\$

OFF ERROR

Dtime $=$ TIMEDATE

DISP " LOADING disk file: ";Filename\$;" ...":

ON ERROR GOTO Bad file

ENTER @Datapath;Status\$

OFF ERROR

ON ERROR GOTO Cant_findfile

SELECT Status\$

CASE " $Y$ " I All graphics/data parameters exist.REN 100,2

DISP "Complete graph." 

ENTER @Datapath;Description\$

ELSE

ENTER @Datapath;Labels $\$($ ),Lbl_count,Lbl_addr(")

ENTER @Datapath;Curve_id\$(*), Symbol\$(*)

ENTER @Datapath;Roster("),Curvecount

ENTER @Datapath;Graphtype \$,Margins $\$(*)$

ENTER @Datapath; X_cross_Y,Y_cross_X

ENTER @Datapath;Xgrid_tick\$,X̄Xmajor,Xminor ENTER @Datapath;Ygrid_tick\$,Ymajor,Yminor ENTER @Datapath;Xmin_graph,Xmax_graph ENTER @Datapath;Ymin_graph,Ymax_graph

CASE " $N$ " I Only data parameters exist.

$$
\text { DISP " RAW data. " }
$$

\section{CASE ELSE}

DISP "Data file is not recognized, entry aborted.": DISP " ...continue."

\section{BEEP}

\section{PAUSE}

\section{OFF ERROR}

GOTO Mistakelineset

\section{END SELECT}

1

ENTER @Datapath;Data_id\$

IF FIg THEN

ENTER @Datapath;Delta_x

ENTER @Datapath;Datacount

Hold_size $=$ Datacount

ENTER @Datapath;Datacount

ENTER @Datapath;Hold_size

END IF

IF NOT Allocated THEN

IF Datacount $>=1$ AND Hold_size $>=1$ THEN

ALLOCATE Holding_file(Hold_size,2)

ELSE

ALLOCATE Holding_file $(1,2)$

END IF

Allocated $=1$

END IF

ENTER @Datapath; Holding_file(*)

ASSIGN @Datapath TO.

OFF ERROR

IF NOT Flg THEN

Delta_ $x=$ Holding_file $(2,1)$-Holding_file $(1,1)$

Strt_time $=$ Holding_file $(1,1)$

$\mathrm{Fig}=1$

END IF

IF Datacount $=0$ THEN Mistakeline

I

(Copy data from Holding_file(") to Basket_file(*)

I

MAT Basket_file $=(0$.

IF Datacount $>$ Basketsize THEN IReceiving file too small. Allocated $=0$ 
3150 Copydatafile:

3152

3154

3156

3158

3160

3162

3164

3166 Mistakelineset:Datacount $=0$

3168 Mistakeline:OFF KEY

3170

3172

3974

3976

3178

3180

3982

3984

3186

3188

3190 Cant_findfile: IError in searching for the file.

3192

3194

3196

3198

3200

3202

3204

3206

3208

3210

3212

3214

3216

3218

3220

3222

3224

3226

3228

IF Allocated THEN DEALLOCATE Holding file ${ }^{*}$ )

LOOP

EXIT IF TIMEDATE-Dtime $>1.8$

END LOOP

DISP CHR\$ 112 )

OUTPUT 2 USING "\#, $K^{n}: " K^{*}$

SUBEXIT

1

I IIIIIIIIIIIIIIIIIIIIIIIIIIIIIIIIIIIIIIIIIIIIIIIIIIIIIIII

!

BEEP 500,.6

SELECT ERRN

\section{CASE 56}

DISP "That file does not exist on this disk ":

CASE $72,73,76,82$

DISP Diskdrive $\$ ;$ " has failed of is not available ":

CASE ELSE

DISP ERRM\$:

END SELECT

DISP " ....CONTINUE to try again."

PAUSE

Filename $\$=$ "

Diskdrive $\$="$ "

GOTO Selectdrive

$$
\text { I }
$$

SUBEND

1

! 


\section{B.4 PULS_PARAMS}

100

102

104

106

108 COM /nterrupts/ INTEGER Intr_prty

110 !

112

114

116

118

120 Intr-prty $=1$

122 CALLL Pulse_params

124

126

128

130

132

134

136

138

140

142

144

$146 \quad$ I

148 Pulse_params: !

150

152

154

156

158

160

162

164

166

168

170

172

174 Dateline:

176

178

180

182

184

186

188

190

192

194

196

198

200

202

204

206

208

CLEAR SCREEN

MASS STORAGE IS ":,1400"

END

1

SUB Pulse_params

OPTION BASE 1

RAD

COM /Time_vals/ Trm 2 _90

COM /Units/ X_units $\$[20], Y$ units $\$[20]$

$$
1
$$

!

l October 12, 1990

! Last revision: May 21,1991

INTEGER Hist_ary(32767),Pnts,Typ

REAL Wave $(3 \overline{2} 767,2)$

DIM Data_id\$[40],Dataname\$[10],Ch\$[1]

Chs $=$ " $y^{n-}$

I

WHILE (Ch\$ $=" Y ")$ OR (Ch\$ $=" y ")$

WAIT 1.0

Diskdrive $\$={ }^{\infty}$ "

Filename $\$="$ "

Pnts $=32767$

REDIM Wave(Pnts, 2)

OUTPUT KBD USING "K, \#":"LOAD KEYE"l restore the typing aid keys

PRINT TABXY $(1,5) ; "$ "END of program. So long."

COM Nolt_vals/ Vmin,Vmax, V_last, Volt_z,Volt_100,V_first, Vptp

COM /Timé_vals/ Tm_10,Tm_20,Tm_50,Tm_80,Tm_90,Tr1_10,Tr1_20

COM $/$ Time_vals/ Tr2_10,Tr2_20,Pls_dur,Tm2_10,Tm2_20,Tm2_50,Tm2_80

COM Misc_int/ INTEGËR No_o_bins,His_zero_lev

COM /Misc_int/ INTEGER His_-100_lev, Maxpoint

COM /Misc_real/ Delta_v,Delta_v_prc,Ov,Undr

COM /Files/ Diskdrive $\$$ [20], Filename $\$$ [14],Ms_path $\$[500$ ]

I This program written by S.M. Chesnut

DISP "Enter the name of the file when prompted." 
258 Mak_histogram: I

260 
424 PRINT "The number of bins in the histogram is less than 128."

426 PRINT "Therefore, the voltage resolution is quite bad and you"

\section{NEXT I}

Hist_ary (Level) $=$ Hist_ary (Level) +1

$$
\text { NEXT I }
$$

Bug1 $=0$

IF Bug1 THEN

PRINTER IS PRT

FOR I $=1$ TO No_o_bins

NEXT I PRINT Hist_ary (I)

ND IF

Bug $1=0$

His_zero_lev $=0$

His_100_lev $=0$

Zipo $=0$

Hundred $=0$

FOR $I=1$ TO Half_bin

IF Hist_ary (I) > His_zero_lev THEN

His_zero_lev $=\bar{H}_{i s t}$ ary (I) Zipo $=1$

END IF

$I=$ Half_bin

WHILE $T<$ No_o_bins

IF Hist_ary $(1+1)>$ His_100_lev THEN

His_100_lev $=$ Hist_ary $(1+1)$

Hundred $=1+9$

END IF

$$
I=I+1
$$

END WHILE

IF Auto THEN

IF (His_zero_lev < Min_bin) THEN No_o_bins $=$ No_o_bins DIV 2 IF No_o_bins $<\overline{1} 2 \overline{8}$ THEN GŌSŪB Hist_message Done $=1$

\section{ELSE}

$$
\text { END IF }
$$

$$
\text { Done }=1
$$

ELSE

$$
\text { Done }=1
$$

END IF

END WHILE

GOSUB Calc_v_prms

GOSUB Hist_query

\section{END WHILE}

SUBEXIT

$436 !$ PRINT "are asked if the histogram is an acceptable one."

WAIT 2.0

RETURN 
438 Calc_v_prms: 1

440 Volt_z $=$ Vmin + Zipo "Delta_v-Delta_v/2.

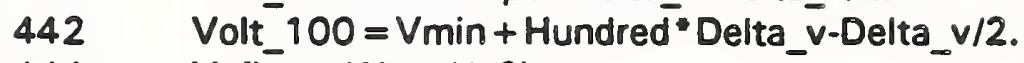

444

446

448

450

452 !

454 Hist_query: !

456 I

458

460

462

464

466

468

470

472

474

476

478

480

482

V_first $=$ Wave $(1,2)$

$\mathrm{V}$-last $=$ Wave $\left(\right.$ Pnts $\left._{0}, 2\right)$

Delta_v_prc $=$ Delta_v $100 /$ ptp

RETURN

484

486

488

490

492

with"

494

496

498

500

502

504

506

508

510

5121

5941

591

518 SUB Vmax_min_ptp(REAL Wave (\%), INTEGER Pnts,REAL Vmax,Vmin,Vptp,INTEGER Maxpoint)

5201

CLEAR SCREEN

PRINT "The first waveform point $={ }^{m} ;$ PROUND(V_first, -4$)_{i}{ }^{n}$ "Y $Y$ units $\$_{i}{ }^{m} . "$

PRINT

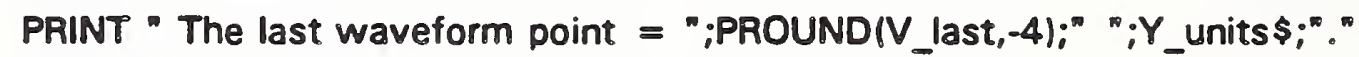

PRINT

PRINT "The minimum $={ }^{m} ;$ PROUND(Vmin, -4$)_{i} "{ }^{*} ; Y_{-}$units $\$ ;^{n}$ "

PRINT

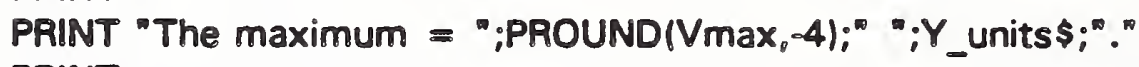

PRINT

PRINT "There were "NNo_o_bins;" bins used in the histogram."

PRINT

PRINT "Each histogram bin is equivalent to" :PROUND(Delta_ $\left.v_{0}-5\right)_{0}^{\text {w }}$ " $; Y_{-}$units $\$_{0}^{*}, "$

PRINT

PRINT "or" "PROUND(Delta_v_prc, -5$)_{i}^{*} \%$ of the waveform peak-to-peak."

PRINT

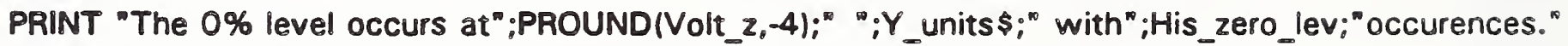

PRINT

PRINT "The 100\% level occurs at ${ }^{n}$ :PROUND(Volt_100,-4):" "Y Y units $\$ ; "$

522 Vmax_min_ptp: !

524 INTEGER I

$526 \quad V_{\max }=W_{\text {ave }}(1,2)$

$528 \quad V \min =$ Wave $(1,2)$

530 FOR I $=1$ TO Pnts

532

534

536

538

540

542

544

546

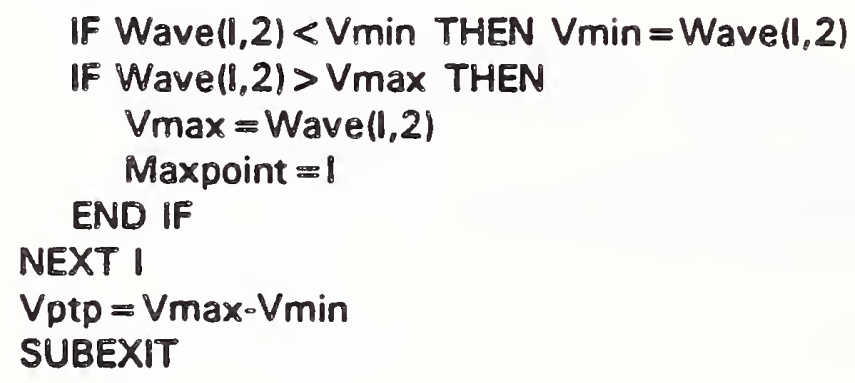


560 I Original: 29 Jun 1987, G. Koepke

562 I Revision: 06 Aug 1987, 10:00

564 OPTION BASE 1

566 DEG

568 COM /Sys/ Sys id $\$[10]$

570 COM /Files/ Diskdrive $\$\left[20\right.$ ], Filename $\$[14], M s \_p a t h \$[500]$ 


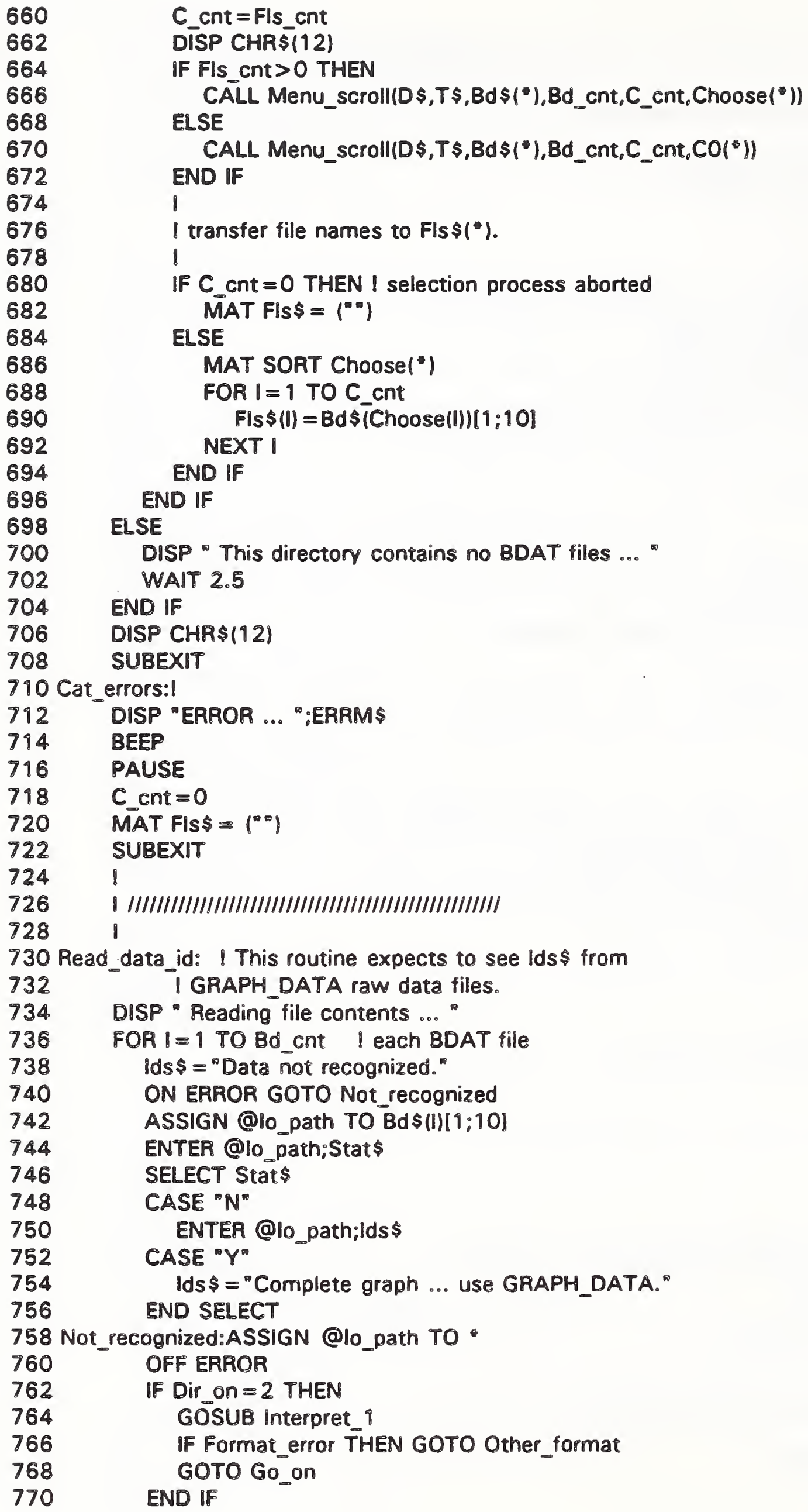


772 Other_format:!

$774 \quad \operatorname{Bd} \$(1)[11,71]=" \ldots$ ".. "\&lds\$

776 Go_on: NEXT I

778 RETURN

$780 \quad 1$

$782 \quad$ I IIIIIIIIIIIIIIIIIIIIIIIIIIIIIIIIIIIIIIIIIIIIIIIIIIII

$784 \quad 1$

786 Interpret_1: I This is used to interpret TEM program ID strings.

788 Format_error $=0$

$790 \quad I$ identify this particular format

792 IF LEN(Ids $\$$ ) $<40$ THEN

794 Format_error $=1$

796 RETURÑ

798 END IF

800 IF Ids $\$[40]<>" *$ " THEN

802 Format_error $=1$

804 RETURN

806 END IF

808 I make the information readable

$810 \quad$ Bd\$(I) $[11,15]=$ " ..."

$812 \quad B d \$(1)[16,25]=\operatorname{ld} s \$[1,10]$

$814 \quad \operatorname{Bd} \$(1)[26,32]="$, " \&lds $\$[11,12] \& "$ :" \&lds $\$[13,14]$

$816 \operatorname{Bd} \$(1)[33,42]="$ " " \&lds $\$[15,16] \& "$ " \&lds $\$[17,18]$ \& " " \&lds $\$[19,20]$

$818 B d \$(I)[43,55]=$ ", " \&lds $\$[21,27] \& " M H z "$

$820 \quad B d \$(I)[56,65]=$ ", " \&lds $\$[28,33] \& " v m "$

$822 \quad B d \$(1)[66,71]=", "$ \& \&lds $\$[38,39]$

824 RETURN

$826 \quad !$

828

830

832 List_directory: I This routine will provide a tabular listing of

834

836

838

840

842

844

846

848

850

852

854

856

858

860

862

864

866

868

870

872

874

876

I the directory along with lds $\$$ if provided
I thectory: This routine will provide a tabular listing of

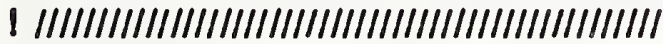
1

DISP " Listing directory ..."

PRINTER IS PRT

PRINT USING " $/ / n$

PRINT T\$

PRINT RPT $\$(" * ", 80)$

PRINT "File name";

IF Dir_on THEN

PRINT " ... contents"

ELSE

PRINT

END IF

PRINT RPT $\$\left("-{ }^{n}, 80\right)$

FOR I = 1 TO Bd_cnt

PRINT Bd\$(I)

NEXT I

PRINT RPT $\$\left({ }^{\circ}{ }_{-}{ }^{\circ} 80\right)$

PRINT

PRINTER IS CRT

RETURN

878

880

882

SUBEND

$$
1
$$

1 
884 SUB Fifty_percent(REAL Wave ("),Wv_50,INTEGER Pnts,Start_pos,Typ)

$886 \quad 1$

888 Fifty_percent: !

$890 \quad 1$

892

894

896

898

900

902

904

906

908

910

912

914

916

$918 !$

920

922

924 DEF FNPUIS_typ(REAL Last,Wv_90)

926 Puls typ: I

928 If (Last $>$ WV 90) THEN

930

932

934

936

938

940

942

944

946 SUB Rev_pol(INTEGER Pnts,REAL Wavel ${ }^{*}, W_{v}, 10, W_{v} 90, W_{v}$ amp. $W_{v}$ base)

948 !

950 Rev pol: I

952

954

956

958

960

962

964

966

968

970

972

974

ELSE

RETURN $\overrightarrow{1}$ step like waveform

RETURN - 1 ! impulse like

ID IF

END

1

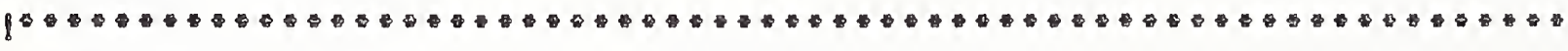

!

!

INTEGER I

FOR $I=1$ TO Pnts

Wave $(1,2)=W v_{-}$amp $+W v_{-}$base-Wave $(1,2)$

NEXT I

SUBEXIT

SUBEND

l

1 -

1

SUB Time_o_prent(REAL Wave ("),Wv_pct,Pct_t,INTEGER Pnts,Drectn,Strt,Typ)

1

978 1 This subroutine assumes all waveforms are positive going, i.e. they

978 I start at on level and increase to another level. If the original

980 I waveform had a negative polarity, this was remedied earlier in

982 I the program.

984 I

986 Time _o_prent: I

988 I imports: wave, an array of data

9901 wr_pct, any desired percentage level

$992 \quad$ pnts, the number of data points

9941 drectn $=1$ if wv pct $>=50 \%$ 


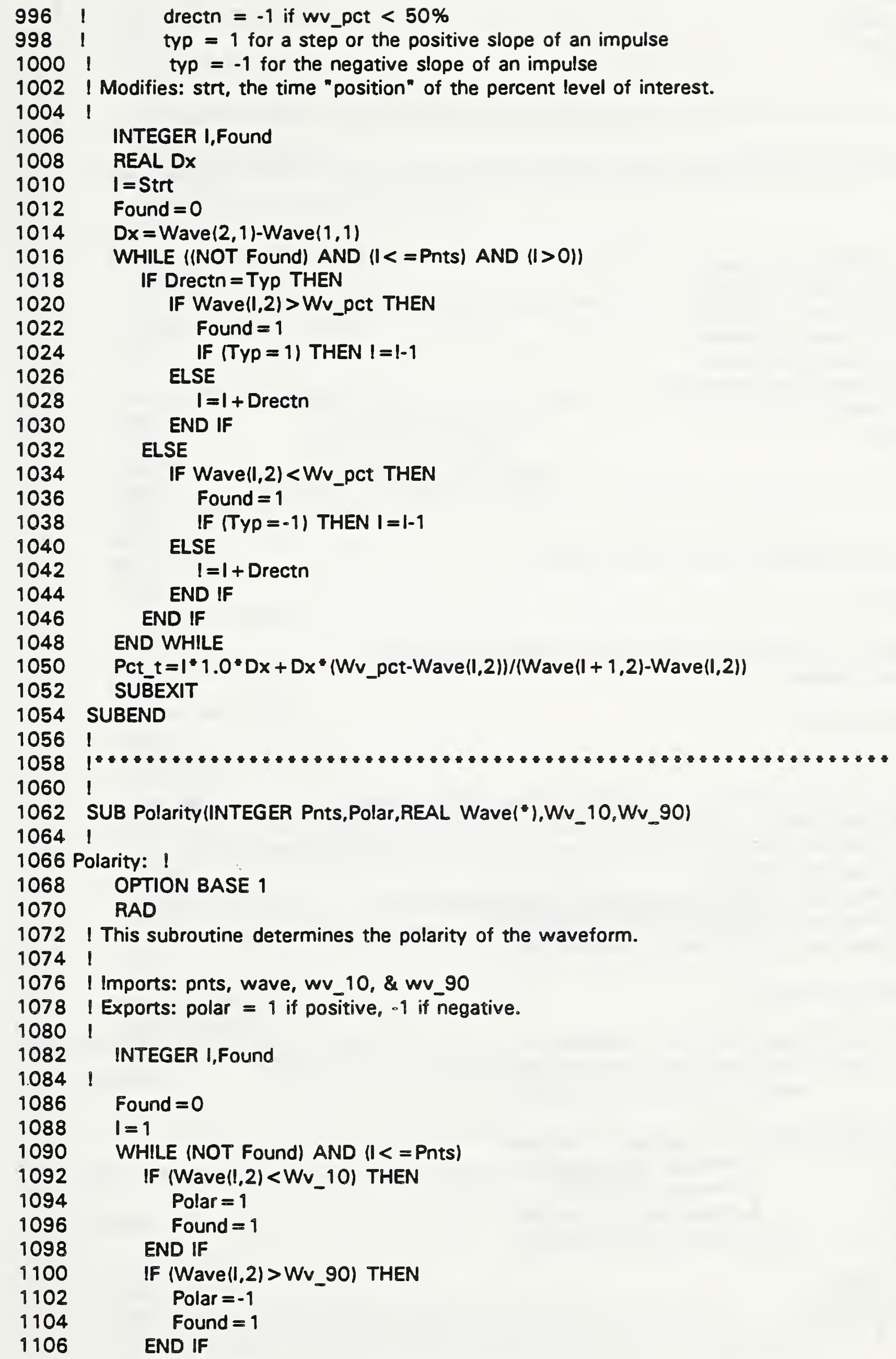




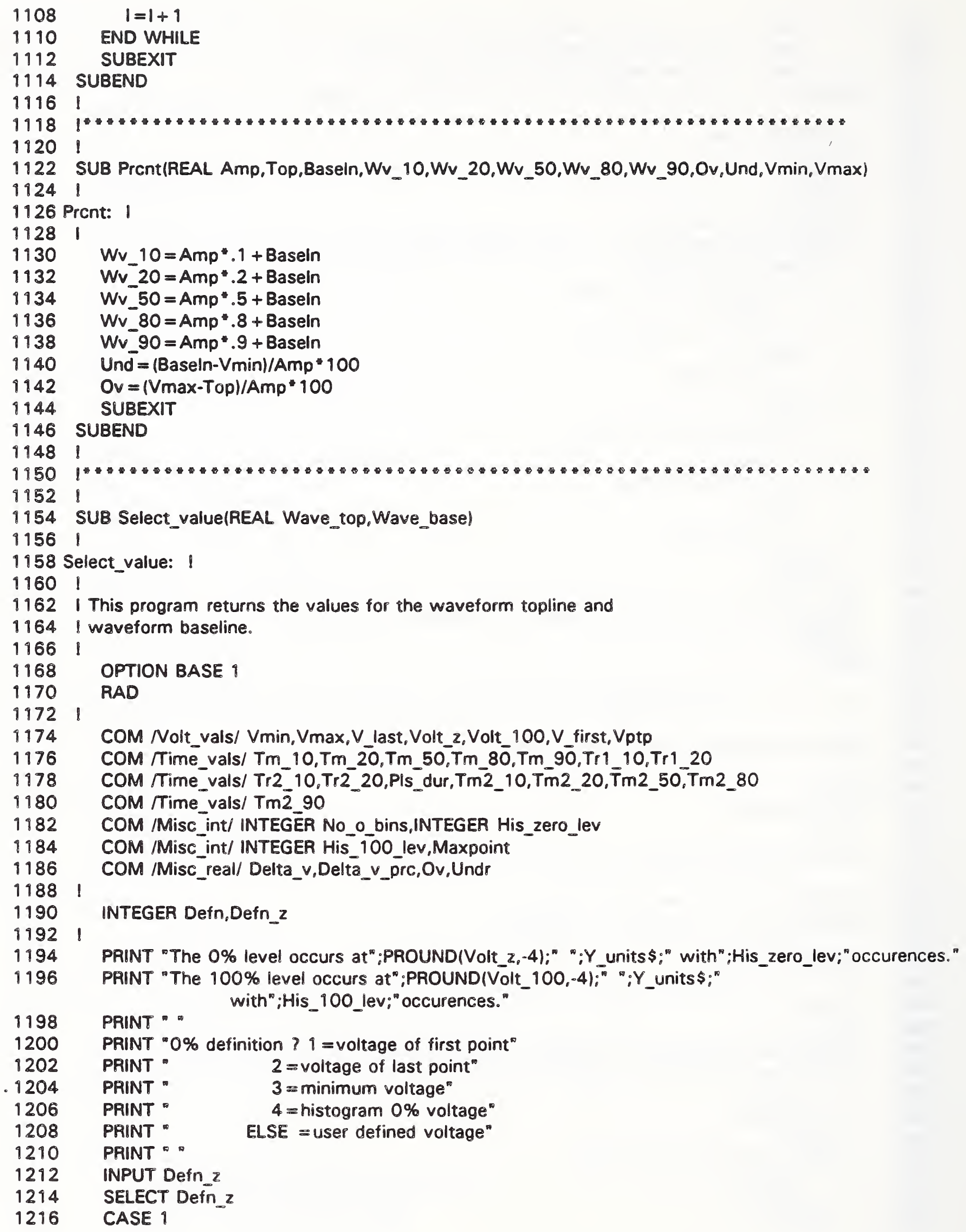

Wv_10 = Amp $.1+$ Baseln

$W v \_20=A m p * .2+$ Baseln

$W v-50=A m p * .5+$ Baseln

$W v \_80=A m p * 8+$ Baseln

$W v \_90=A m p * .9+$ Baseln

$\mathrm{Und}^{-}=($Baseln-Vmin $) / A m p=100$

$O v=(V \max -T o p) / A m p * 100$

SUBEXIT

SUBEND 
$1316 \quad$

1318 Parameters: I

1320

1322 


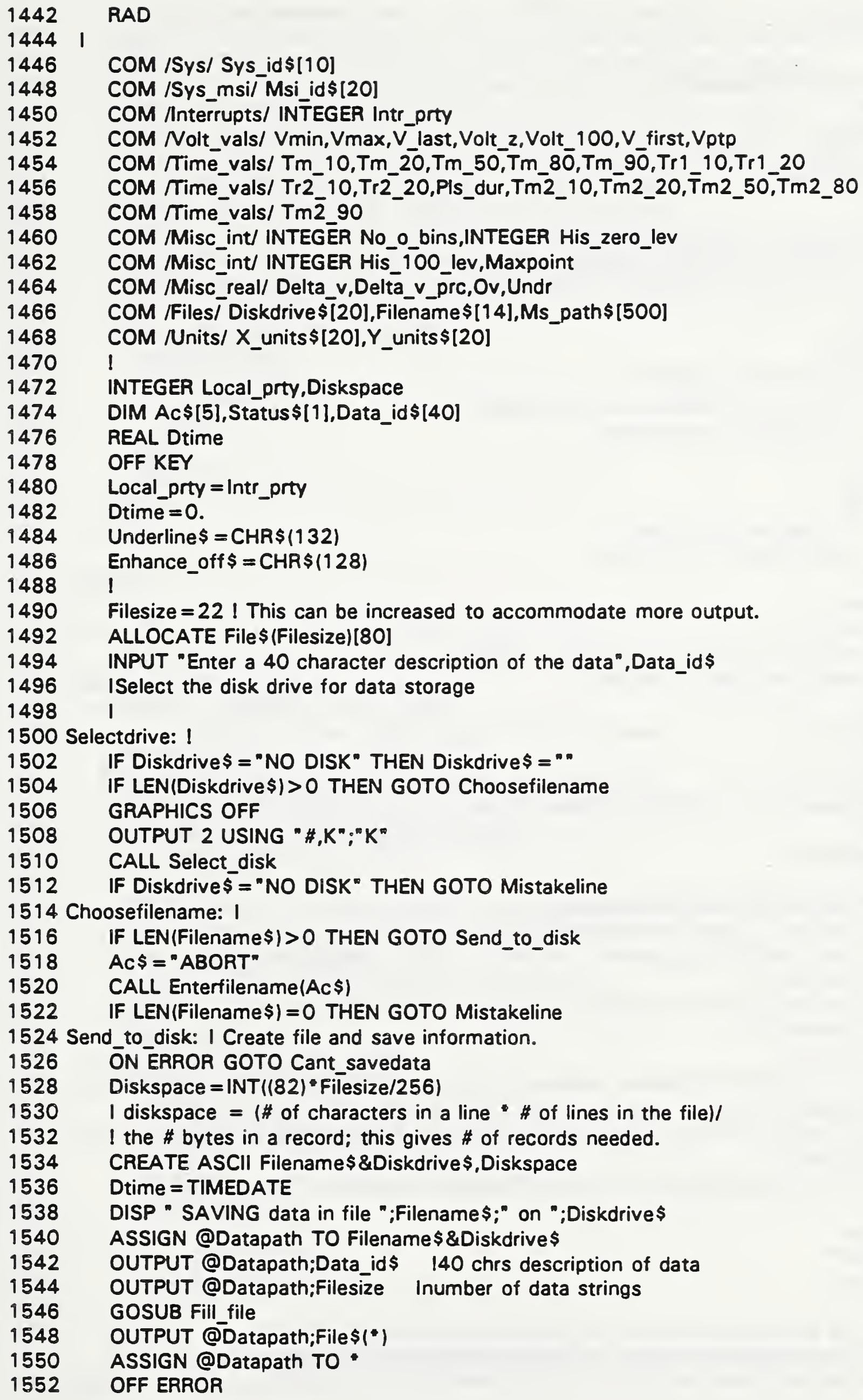




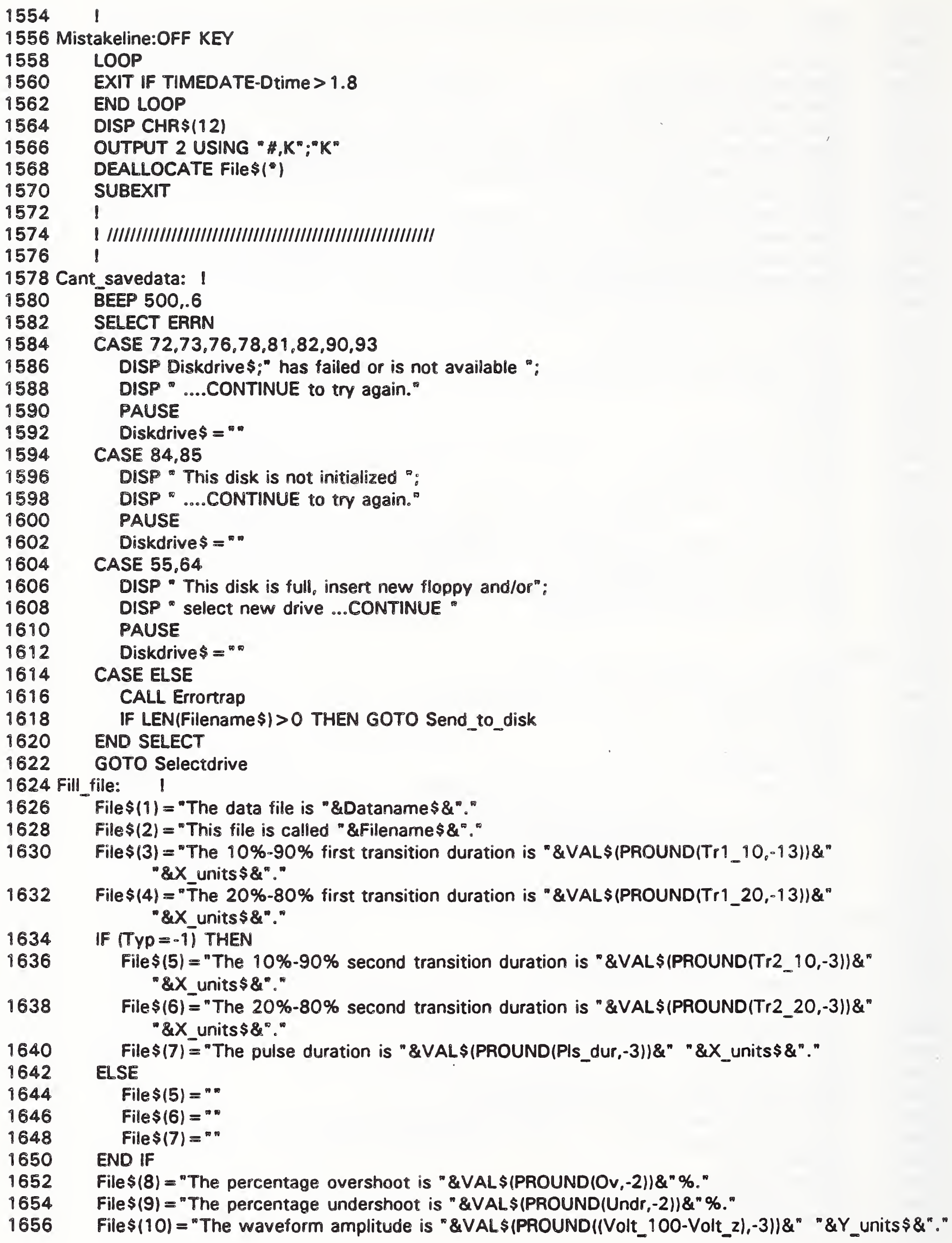


File $\$(11)=$ "The maximum level is "\&VAL $\$(P R O U N D(V \max ,-3)) \& "$ "\&Y_units $\$ \& " . "$ File $\$(12)=$ "The minimum level is "\&VAL\$(PROUND(Vmin,-3)ו\&" "\&Y_units\$\&"." File $\$(13)=$ "There were "\&VAL\$(No_0_bins)\&" bins used in the histogram." File $\$(14)=$ "Each histogram bin is equivalent to "\&VAL\$(PROUND(Delta_v,-6)/\&" "\&Y_units $\$$ \&"."

1712 SUB Enterfilename(Ac\$)

1714 Enterfilename: I Original: 13 Nov 1984 OPTION BASE 1

! Revision: 10 Dec 1990 includes HFS directories

COM /Files/ Diskdrive $\$[20]$,Filename $\$[14], M s$ path $\$[500]$

COM /nterrupts/ INTEGER Intr_prty

INTEGER I,Ascii_num,Maskflag,Namelength

DIM Test\$[256],Hfs_temp\$[161]

Namelength $=10$

IF LEN(Ms_path\$) >0 THEN OUTPUT KBD USING "K, \#";" \#" \&Ms_path\$\&" $H^{n}$ DISP " ENTER HFS directory PATH (no file)":

IF AC $\$<>$ "PATH" THEN

DISP ", ENTER / for HFS ROOT or null for LIF..." ; END IF

LINPUT Hfs_temp\$

His_temp $\$=$ TRIM $\$$ (Hfs_temp\$)

IF LEN(Hfs_temp\$1>0 THEN

IF LEN(Hfs_temp\$) $>1$ AND Hfs_temp\$[LEN(Hfs_temp $\$) ; 1]<>" /$ THEN

END IF

Hfs_temp $\$=$ Hifs_temp $\$ \&^{\prime \prime} / "$

IF LEN(Hfs_temp\$) $=1$ THEN Hfs_temp $\$=$ " "

Namelengt $\bar{h}=14$

END IF

IF AC $\$=$ "PATH" THEN

Ms_path\$ $=$ Hfs_temp\$

END IF

1766 IF LEN(Filename $\$$ ) >0 THEN OUTPUT KBD USING $" K, \#^{n} ;^{n} \#^{n}$ \&Filename $\$ \&^{n} H^{n}$

1768 Efn: ! 
2054 Diskselected:OFF KEY

2056

2058

2060

2062

2064

2066

2068 File

2070

2072

2074

2076

2078

2080

2082

2084

2086

2088

2090

2092

2094

2096

2098

2100

2102

2904

1

Disc $\$(D d+1)=": 702,0$ - HP 9122 dual microfloppy left drive"

Disc $\$(D d+2)=":, 702.1$ - HP 9122 dual microfloppy right drive"

Disc $\$(D d+3)=: 703,0 \quad$ - HP 9125 single 5.25 floppy drive

Disc $\$(D d+4)=": 1400$ - HP $9133 \mathrm{H}$ hard disk volume I" $^{*}$ I

$D d=D d+4 \quad l$ add the number of drive specifiers above

l

IF Sys_id $\$[1,4]<>$ "S300" THEN

Disc $\$(D d+1)=": 4,1 \quad$ - LEFT internal series $200^{\circ}$

Disc $\$(D d+2)=":, 4,0 \quad-$ RIGHT internal series $200^{\circ}$

$\mathrm{Dd}=\mathrm{Dd}+2$

END IF

1.

I

CALL Menu_scroll(Displ\$,Title\$,Disc \$("),Dd,Pt,Choose("))

IF Pt $=0$ THEN

Diskdrive $\$=$ "NO DISK"

\section{ELSE}

Dd $=$ POS $\left(\right.$ Disc $\left.\$(\text { Choose }(P t))_{\theta} "{ }^{m}\right)-1 \quad 1$ find.

If Dd $>5$ THEN \& valid msus ELSE

Diskdrive $=$ TRIM \$ (Disc \$ (Choose(Pt)) [1,Dd])

DISP " ERROR in reading MSUS from string, - chr not found. "

BEEP

CALL Pause key_on

Diskdrive $\$=$ "NO DISK"

END IF

END IF

SUBEXIT

SUBEND

1

SUB File_menu(Mask\$,Ftype $\$, F I s \$(*)$, INTEGER Fls_cnt,Dir_on,Prton)

le menu: \&

I Original: 29 Jun 1987, G. Koepke

I Revision: 02 Dec 1987, 07:00

OPTION BASE 1

DEG

COM /Sys/ Sys id \$110]

COM /Files/ Diskdrive \$[20],Filename\$ [14],Ms_path\$[500]

COM /interrupts/ INTEGER Intr_prty

COM /Bugs/ INTEGER Bug 1,Bug2,Bug3,Printer

DIM Directory $\$(600)[80\}, B d \$(600)[71]$

DIM D\$[80],T\$[51],Ids\$[40],Stat\$[1],Test\$[256]

INTEGER Bd_cnt,File_cnt,l,C_cnt,CO(1),Format_error,End_search

IF Fis_cnt $>0$ THEN ÄLLOCATTE INTEGER Choose(Fls_cnt)

1

I Catalog the disk specified

I

End search $=0$

REPEAT I Generate path to file and extract file name.

ON ERROR GOTO Cat_errors 
DISP " Reading the Directory ..."

IF LEN(Ms_path\$) >0 THEN

MASS STORAGE IS Ms_path\$[1,LEN(Ms_path\$)-1]\&Diskdrive $\$$ ELSE

MASS STORAGE IS Diskdrive\$

\section{END IF}

CAT TO Directory $\$(*)$;NO HEADER,COUNT File_cnt

\section{OFF ERROR}

1

I set up array of legal file names.

1

Bd_cnt $=0$

MATT Bd\$ $=(" n)$

FOR $I=1$ TO File_cnt

SELECT Directory $\$(1)[32,36]$

CASE Ftype\$ I Ftype $\$=$ "BDAT" or

I Ftype $\$=$ "PROG "

IF LEN(Mask\$) >0 THEN ! Test for mask\$

IF Directory $\$(\mid)[1$, LEN(Mask\$) $]=$ Mask $\$$ THEN

Bd_cnt $=$ Bd_cnt +1 END IF

$\operatorname{Bd} \bar{s}\left(B d \_c n t\right)=$ Directory $\$(1)[1 ; 14] \& "$ - "\&Ftype $\$$

ELSE

$B d \_c n t=B d \_c n t+1$

$B d \overline{\$}\left(B d \_c n t\right)=$ Directory $\$(1)[1 ; 14] \& "$. "\&Ftype $\$$

END IF

CASE "DIR " 1 plus all "DIR " listings

Bd_cnt $=$ Bd_cnt +1

Bd $\overline{\$}\left(B d \_C n t\right)=$ Directory $\$(1)[1 ; 14] \& "$ - DIR "

\section{CASE ELSE}

END SELECT

\section{NEXT I}

IF LEN(Ms_path\$) $>0$ AND Bd_cnt $>0$ AND Fls_cnt $>0$ THEN

Bd_cnt $=B d \_c n t+1$

Bd $\overline{\$}\left(B d \_c n t\right)="$ - MOVE back up ONE Directory level."

Bd_cnt $=$ Bd_cnt +1

$\mathrm{Bd} \overline{\mathrm{S}}\left(\mathrm{Bd} \_\mathrm{cnt}\right)^{-}=$" - RETURN to ROOT Directory."

END IF

1

I set up file menu

I

D\$ = "Select "\&VAL\$(Fls_cnt)\&" file name(s) for data entry."

$T \$=$ "List of "\&Ftype $\$$ " files and DIRs on "\&Diskdrive $\$$

IF LEN(Mask\$) $>0$ THEN

$T \$=T \$ \& "$ mask $=$ " \&Mask $\$$

END IF

IF Bd_cnt $>0$ THEN

IF Dir_on $>0$ THEN GOSUB Read_data_id

IF Prt on THEN

GOSUB List_directory

ELSE

$$
\text { End_search }=1
$$

C_cnt $=$ Fls_cnt

DISP CHRS(12)

IF FIs cnt $>0$ THEN

CALLL Menu_scroll(D\$,T\$,Bd\$("),Bd_cnt,C_cnt,Choose (")) 
2332 Read_data_id: I This routine expects to see Ids\$ from I GRAPH_DATA raw data files.

2368 Not_recognized:ASSIGN @lo_path TO.

2382 Other_format:I

DISP " Reading file contents ... Please stand by. "

PRINT TABXY $(1,18) ; "$ Reading \#":

FOR $\mid=1$ TO Bd cnt $\mid$ each BDAT file PRINT TABXȲ $(11,18)$;

PRINT USING "3D,4A,3D,2A,\#" ;1," of "Bd_cnt,". "

Ids $\$=$ "Data not recognized."

IF Bd\$(I) $[18,22]=$ "BDAT " THEN

ON ERROR GOTO Not_recognized

ASSIGN @lo_path TO Bd\$(I) $[1 ; 14]$

ENTER @lo_path;Stats

SELECT Stat\$

CASE " $N$ "

ENTER @lo_path;Ids\$

$$
\text { CASE " } Y \text { " }
$$

Ids $\$=$ "Complete graph in GRAPH_DATA form."

\section{END SELECT}

OFF ERROR

IF Dir_on $=2$ THEN

GOSUB Interpret 1

IF Format_error THEN GOTO Other_format GOTO Go_on

2384

2386

2388 Go_on:NEXT I

2390

2392

2394

2396

2398

2400

2402 Interpret_1: I This is used to interpret ID strings.

2404 Format_error $=1$

2406

2408

2410

2412

2414

2416 List_directory: I This routine will provide a tabular listing of

2418

2420

2422

2424

2426

2428

2430

2432

2434

2436

2438

2440

PRINT TABXY(1,18);RPT \$(" ",40);

DISP CHR\$ (12);

RETURN

1

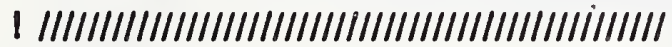

1

$$
1 \text { identify this particular format }
$$

RETURN

1

\section{[ IIIIIIIIIIIIIIIIIIIIIIIIIIIIIIIIIIIIIIIIIIIIIIIIII} 1

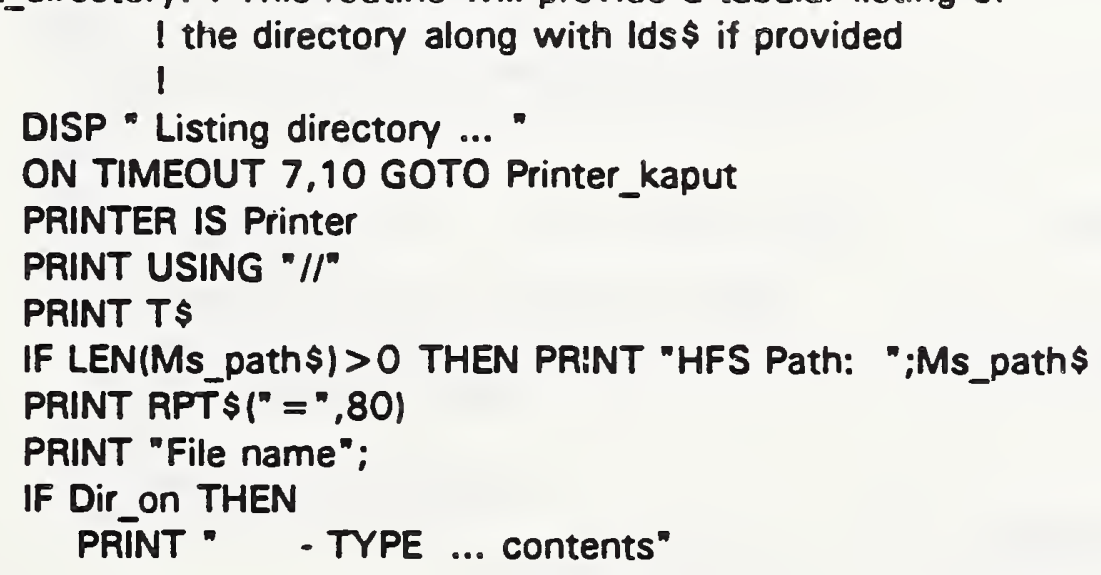


ELSE

2488 SUB Menu_scroll(D\$,T\$,Items \$ ("),INTEGER Item_cnt, To_select,Choose (")

2490 Menu_scroll:! Original: 22 Jun 1987, Galen Koepke. NBS 723.04 
2556 Def_variables:I

2558 COM Interrupts/ INTEGER Intr_prty

2560 COM /Bugs/ INTEGER Bug1,Bug2,Bug3, Printer

2562 COM /Sys/ Sys_id\$[10]

2564 I INTEGER Screen_cnt, Items_per_scn,First_item(40),Last_item(40) INTEGER I,J,K,First_line,Last_line,_Active_screen,Pointer,Last_pt INTEGER Local_prty,Skips,Knobcount,Pointeractive,KO,Null_file INTEGER Exit_flag,Temp,Random_select,Indx DIM Marker $\$[\overline{8}]$, Test\$[256] 1

I initialize parameters 1 Local_prty $=$ Intr_prty IF Local_prty $<1$ THEN Local_prty $=10$

2632 Define_screens:! Set up screens of 15 items each. 


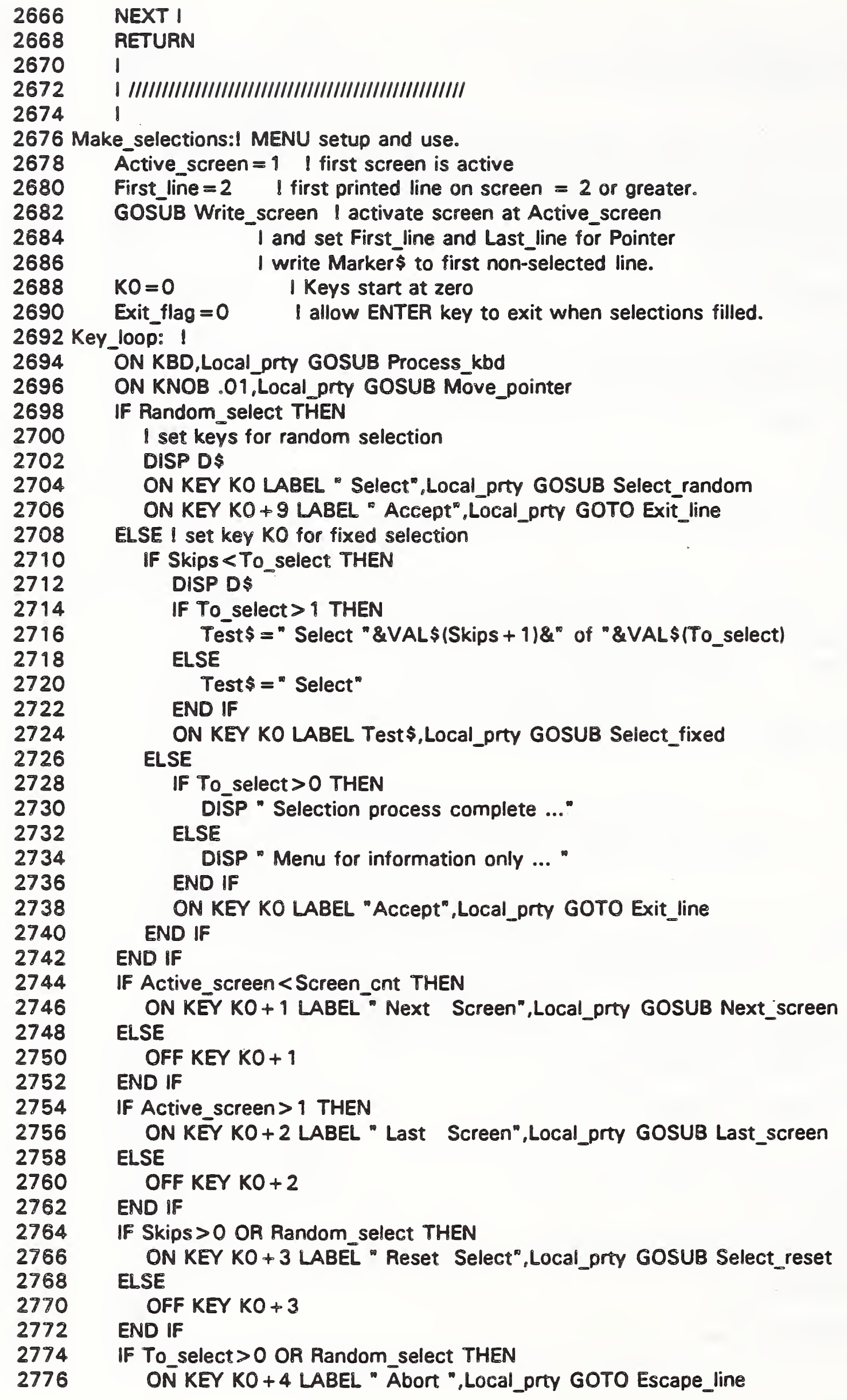

DISP D\$

IF To_select $>1$ THEN Test $\$=$ " Select "\&VAL\$(Skips +1$) \&$ " of " \&VAL\$(To_select) 
ELSE

END IF

IF Screen_cnt $>2$ THEN ON KEY KO +6 LABEL "Jump to Screen",Local_prty GOSUB Jump_to_scn ELSE

OFF KEY KO +6

END IF

IF Exit_flag THEN Exit_line

GOTO Key_loop

Scape_line:Skips $=0$

$$
\text { MÁT Choose }=(0)
$$

2804 Exit

2806

2808

2810

2812

2814

2816

2818

2820

2822

2824

2826 Next_screen: ।

2828

2830

2832

2834

2836

2838

2840

2842

2844

2846

2848 !

2848 Last_screen:

2850 OFF KBD

2852 OFF KNOB

2854 OFF KEY

2856 IF Active_screen $=1$ THEN RETURN

2858 Active_screen = Active_screen-1 ,

2860

2862

2864

2866

2868

2870 Jump_to_errors:DISP " Not a valid screen number ... try again. "

2872 BEEP

2874 WAIT 1.8

2876 Jump_to_scn: !

2878 OFF KBD

2880 OFF KNOB

2882 OFF KEY

2884 DISP " ENTER the screen number desired ( 1 to ";Screen_cnt;").";

2886 LINPUT Test\$

2888 Test $\$=$ TRIM $\$($ Test $\$)$ 
IF LEN(Test \$) =0 THEN Jump_to_return

ON ERROR GOTO Jump_to_errors

Temp = INT(VAL(Test $\$$ )

OFF ERROR

IF Temp $<1$ OR Temp > Screen_cnt THEN Jump_to_errors

Active screen $=$ Temp

2904 Jump_to peturn: !

2918 Select fixed:! 
3042 Select_random:I

3044 OFF KBD

3046 OFF KNOB

3048 OFF KEY

3050

3052

3054

3056

3058

3060

3062

3064

3066 PRINT CHR\$(1 29$) ; 1$ inverse video 


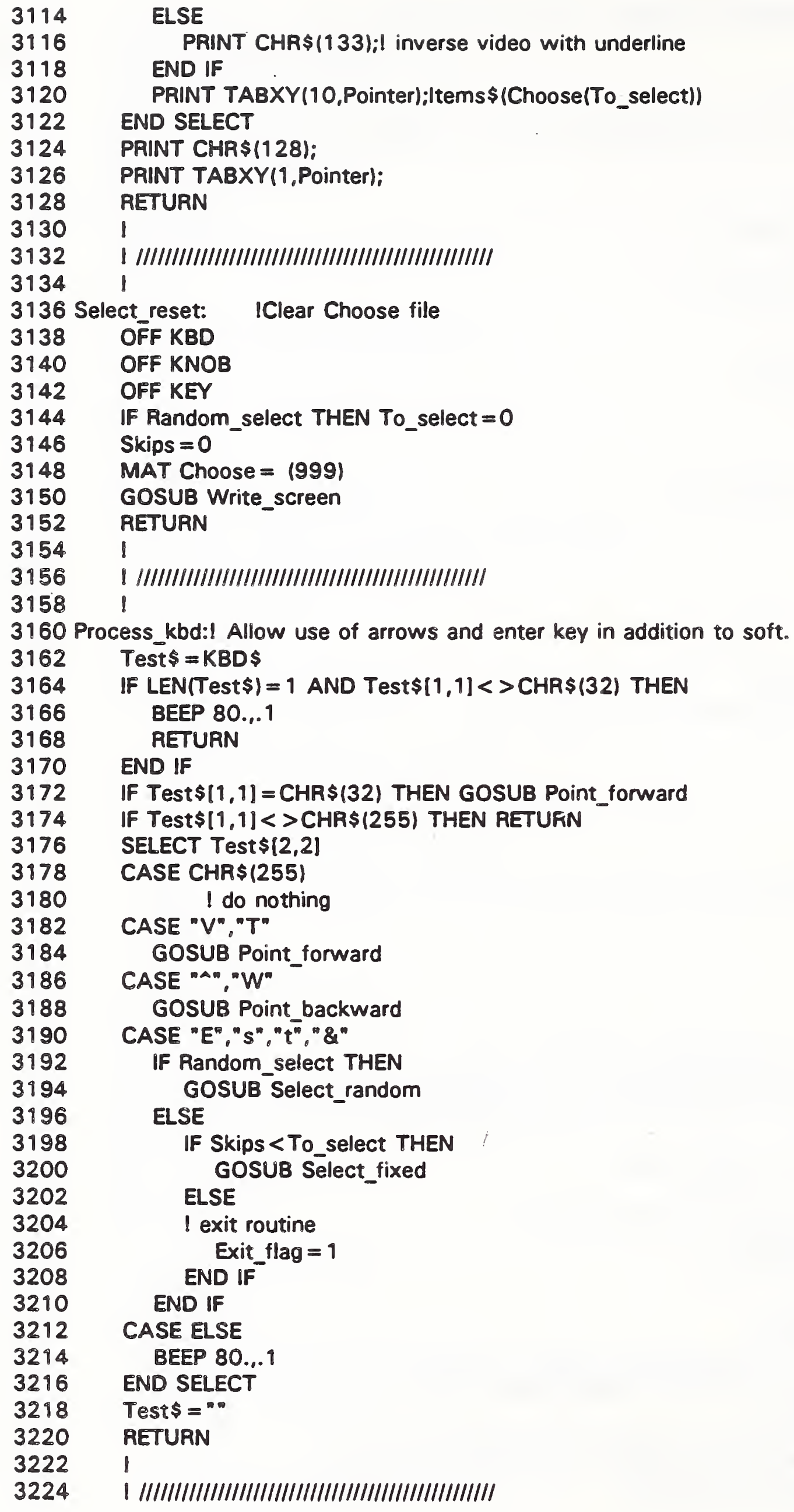


3228 Point_forward:Knobcount $=5$

3230 ḠOSUB Move_pointer

3232 RETURN

3234 Point backward:Knobcount $=-5$

3236 GOSUB Move_pointer

3238 RETURN

$3240 \quad 1$

$3242 \quad$ I IIIIIIIIIIIIIIIIIIIIIIIIIIIIIIIIIIIIIIIIIIIIIIIIIII

$3244 \quad !$

3246 Jog_pointer:I Move the selection pointer on the active screen.

$3248 \quad$ I without regard to selected values

3250 IF Knobcount $>0$ THEN I Move forward

$3252 \quad$ Pointer $=$ Pointer +1

3254

3256

3258

3260

3262

3264

3266

3268

3270

3272 Move_pointer:l Control pointer to avoid re-selection of items

3274

3276

3278

3280

3282

3284

3286

3288

3290

3292

3294

3296

3298

3300

3302

3304

3306

3308

ELSE

Pointer $=$ Pointer -1

I Move backward

END IF

IF Pointer < First_line THEN Pointer = Last_line

IF Pointer $>$ Last_line THEN Pointer $=$ First_line

RETURN

1

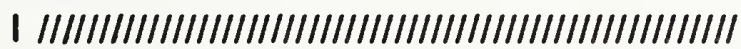

!

IF NOT Pointeractive THEN RETURN I No selections to be made.

Knobcount $=$ Knobcount + KNOBX-KNOBY

IF ABS(Knobcount) $<4$ THEN RETURN

Last_pt $=$ Pointer

GOSUB Jog_pointer

IF Skips $>0$ THEN

LOOP

$J=$ Pointer-First_line

FOR $I=1$ TO Sḱps

IF First_item(Active_screen) $+\mathrm{J}=$ Choose(I) THEN $J=999$ NEXT I

IF $\mathrm{J}=999$ AND Pointer $=$ Last $\_$pt THEN Pointeractive $=0$

EXIT IF Pointeractive $=0$

IF $J=999$ THEN GOSUB Jog_pointer

EXIT IF $\mathrm{J}<>999$

END LOOP

END IF

Knobcount $=0$

3310

3312

3314

3316

3318

3320

3322

3324

3326

3328

3330

3332

3334

3336

OUTPUT KBD;CHR\$(255)\&CHR\$(84); I Bring screen home

IF Last_pt = Last_line THEN PRINT CHR\$(132):

PRINT " ":

IF Pointeractive THEN I Pointer active

IF Pointer = Last_line THEN PRINT CHR\$(132);

ELSE

PRINT CHR\$(128);

END IF

PRINT TABXY (1,Pointer);Marker\$;CHR\$(128);

END IF

RETURN

1

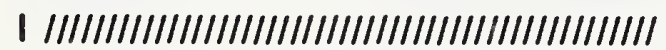


3340 Write_screen:I Write the screen pointed to by Active_screen

3342 I home and clear screen

3344 OUTPUT KBD;CHR\$(255)\&CHR\$(84)\&CHR\$(255)\&CHR\$(75);

3346 Knobcount $=$ KNOBX-KNOBY I Clear knob and keyboard

$3348 \quad$ Knobcount $=0$

3350 Test $\$=$ KBD $\$$

3352 Test $\$=" n$

$3354 \quad 1$

3356

3358

3360

PRINT TABXY(1,First line-1);CHR\$(132):" Item \#| Screen \#";

3362

3364

3366

3368

3370

3372

3374

3376

3378

3380

3382

3384

3386

3388

3390

3392

3394

3396

3398

3400

3402

3404

3406

3408

3410

3412

3414

3416

3418

3420

3422

3424

3426

3428

3430

3432

PRINT USING "\#,2D,4A,2D,3A";Active_screen," of ";Screen_cnt;" $\mid$ "

PRINT T\$;RPT \$(" ",51-LEN(T\$));

PRINT TABXY(80,First_line-1);" $\mid "$;CHR\$(128):

$J=0$

REPEAT

IF $J=$ Last_item(Active_screen)-First_item(Active_screen) THEN PRINT CHR\$ (132);

PRINT TABXY(1,First_line + J);RPT $\$\left({ }^{\circ} ", 80\right)$

ELSE

PRINT CHR\$(128):

END IF

PRINT TABXY(5, First line + J):

PRINT USING "3D,A, "W" :First_item(Active_screen) $+J, " 1 "$

IF Random select THEN

FOR $I=1$ TO To_select

IF First_item(A) $\bar{A}$ ctive_screen) $+J=$ Choose(l) THEN

END IF

NEXT I

ELSE

IF Skips $>0$ THEN $\&$ make this line inverse video

FOR $I=1$ TO Skips

IF First_item(Active_screen) $+J=$ Choose(I) THEN

PRINT CHR\$(12)

END IF

NEXT I

END IF

END IF

PRINT TABXY(10,First_line $+J)$;ltems $\$$ (First_item(Active_screen) $+J)$

PRINT TABXY $(80$, First_line $+J):^{n} 1^{\infty}$;

$J=J+9$

UNTIL $J>=$ (Last_item(Active_screen)-First_item(Active_screen) +1 )

Last_line $=$ Last_item (Active_screen)-First_item (Active_screen)

Last_line $=$ Last_line + First_line

l

I set marker to first non-selected item.

1

Pointeractive $=0$

IF To_select $>0$ OR Random_select THEN Pointeractive $=1$

IF Skips $>0$ AND Pointeractive $=1$ THEN ! find first non-selected item $J=0$

LOOP

Pointer $=$ First line $+J$

FOR $1=q$ TO Skips

IF First_item(Active_screen) $+\mathrm{J}=$ Choose(I) THEN Pointer $=0$ NEXT । 


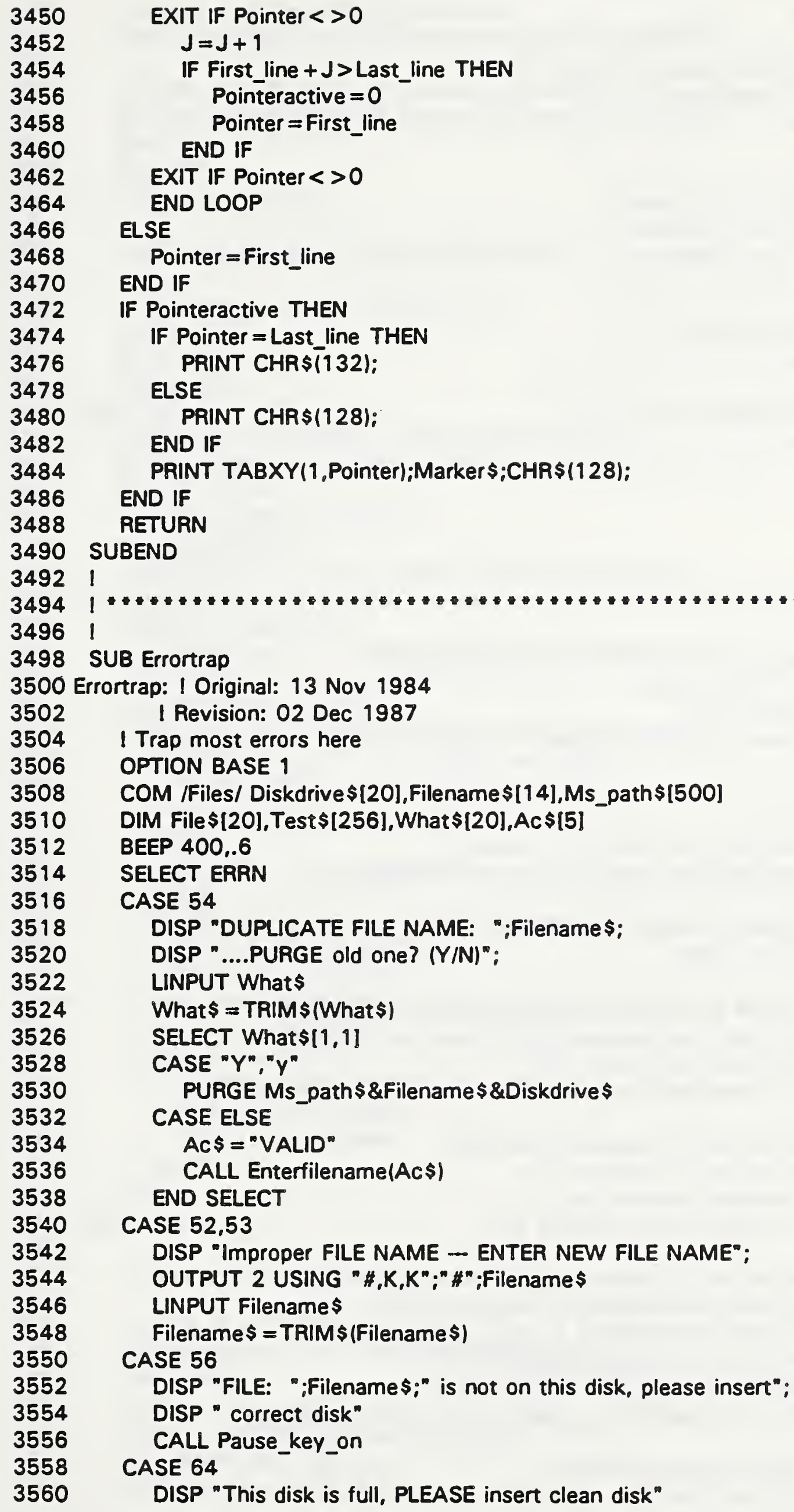

3498 SUB Errortrap

3500 Errortrap: I Original: 13 Nov 1984

3502 I Revision: 02 Dec 1987

3504 1 Trap most errors here OPTION BASE 1

END IF END IF $J=J+1$

IF First_line $+J>$ Last_line THEN Pointeractive $=0$ Pointer $=$ First_line

EXIT IF Pointer $<>0$ END LOOP

ELSE

END IF

IF Pointeractive THEN

IF Pointer = Last_line THEN

ELSE

PRINT CHR\$(132);

END IF

PRINT TABXY(1,Pointer);Marker\$;CHR\$(128);

RETURN

BEND

1

COM /Files/ Diskdrive $\$[20]$,Filename $\$[14], M s$ path $\$[500]$

DIM File\$[20],Test\$[256], What\$[20],Ac\$[5]

BEEP 400,.6

SELECT ERRN

CASE 54

DISP "DUPLICATE FILE NAME: ";Filename\$;

DISP "....PURGE old one? (Y/N)":

LINPUT What $\$$

What $\$=$ TRIM $\$$ (What $\$$ )

SELECT What $\$[1,1]$

CASE " $Y$ ", $" Y$ "

PURGE Ms_path $\$$ \&Filename $\$ \&$ Diskdrive $\$$

CASE ELSE

AC $\$=$ "VALID"

CALL Enterfilename(Ac\$)

END SELECT

CASE 52,53

DISP "Improper FILE NAME - ENTER NEW FILE NAME";

OUTPUT 2 USING "\#,K,K";" \#";Filename\$

LINPUT Filename\$

Filename $\$=$ TRIM $\$$ (Filename $\$$ )

CASE 56

DISP "FILE: ";Filename $\$ ; "$ is not on this disk, please insert";

DISP " correct disk"

CALL Pause_key_on

CASE 64

DISP "This disk is full, PLEASE insert clean disk" 


\section{CASE 56}

DISP "DATA INPUT disk must be in drivell ": DISP "...CONTINUE when ready."

CALL Pause_key_on

CASE $72,73,7 \overline{6}$

DISP Diskdrive\$:

DISP " is not available, type correct":

DISP " unit specifier (ie. ${ }^{\circ}: 707,0 \%$ \%":

OUTPUT 2 USING "K,"\#":Diskdrive\$

LINPUT Diskdrive\$

\section{CASE 80}

DISP "CHECK DISK drive doorl"

CALL Pause_key_on

CASE ELSE

DISP ERRMS;" "CONTINUE' when fixed"

CALL Pause_key_on

\section{END SELECT}

DISP CHR\$(12)

SUBEXIT

\section{SUBEND}

!

1

SUB Load_disk_data(Basket_file( ${ }^{*}$ ),INTEGER Basketsize,Data_id\$)

Load_disk_data: I Original: $1 \overline{3}$ Nov 1984

I Revision: 02 Dec 1987

IThis routine will enter data files from the disk

OPTION BASE 1

1

COM /Sys/ Sys id\$

COM /History/ Status $\$[1]$,Time_orgn\$[8],Date_orgn $\$[11$ 1]

COM /History/ Time_chng\$[8],Date_chng\$[11],Description\$[160] 1

COM Rabels/ Labels $\$(30)\{60], I N T E G E R$ Lbl_count,REAL Lbi_addr $(30,6)$

ILbi_addr: $x, y$, pen, size, LDIR, LORG !

COM /Data_param/ INTEGER Datacount,Filesize,Curvecount,Roster $(17,4)$

COM/Data_param/REAL Sym_size,Symbol\$(17)[2],Curve_id\$(17)[40]

COM /Data_param/REAL Xmin_data,Xmax_data

COM/Data_param/ REAL Ymin_data,Ymax_data

IRoster: Curve\#, Start Addr in File( ${ }^{*}$ ), Datacount, and PEN

ISymbol\$ $(i)={ }^{n \text { " or }}$ " $Y " \Rightarrow$ no symbol, connect pts

ISymbol $\{i\}={ }^{\circ} Y^{*} \Rightarrow>$ symbol, connect pts

ISymbol $\$(i)="{ }^{*}=>$ symbol, do not connect pts 1

COM /Background/ Graphtype \$[12],Margins $\$(2)[10], P a p e r s i z e \$[1]$

COM /Background/REAL Pen_speed,INTEGER Backgnd_pen,Auto_time

COM /Background/ INTEGER Auto_file, REAL X_cross_y, $Y_{-}$cross_ $x$

COM /Background/ Xgrid_tick\$[4],INTEGER Xmajor, Xminor

COM /Background/Ygrid_tick\$[4],INTEGER Ymajor,Yminor

COM /Background/REAL Xmin_graph,Xmax_graph,Ymin_graph,Ymax_graph

\section{!}

COM /Bugs/ INTEGER Bug 1,Bug2,Bug3,Printer

COM /Interrupts/ INTEGER Intr_prty 
INTEGER R,Hold_size,Local_prty,Allocated,FIs_cnt

3706 IF Diskdrive $\$=$ "NO DISK" THEN Diskdrive $\$=$ " "

3708 IF LEN(Diskdrive\$1>0 THEN GOTO Choosefilename

3710 GRAPHICS OFF

3712 OUTPUT 2 USING "\#,K";"K"

3714 CALL Select_disk

3718 Choosefilename: !

3720 Tempfile $\$=$ Filename $\$$

3722 IF LEN(Filename $\$$ ) $>0$ THEN GOTO Bring_in_data

3724 AC $\$=$ "CAT" 
OFF ERROR

ON ERROR GOTO Cant_findfile

SELECT Status\$

3792

CASE "Y" I All graphics/data parameters exist.REN 100,2

3794

3796

3798

3800

3802

3804

3806

3808

DISP " Complete graph.

ENTER @Datapath;Time_orgn\$,Date_orgn\$

ENTER @Datapath;Time_chng\$,Date_chng\$

ENTER @Datapath;Description\$

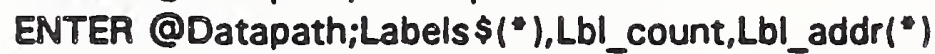

ENTER @Datapath;Curve_id $\$(")$, Symbol $\$(*)$

ENTER @Datapath; Roster("),Curvecount

ENTER @Datapath; Graphtype $\$$, Margins $\$(")$

ENTER @Datapath; X_cross_Y,Y_cross_X

3894

3816

3898

3820

3822

3824

3826

3828

3830

3832

3834

3836

3838

3840

3842

3844

3846

3848

3850

3852

3854

3856

3858

3860

3862

3864

3866

3868

3870

3872

3874

3876

3878

3880

ENTER @Datapath;Xgrid_tick\$, $\bar{X}$ major,Xminor

ENTER @Datapath;Ygrid_tick\$,Ymajor,Yminor

ENTER @Datapath;Xmin_graph,Xmax_graph

ENTER @Datapath;Ymin_graph,Ymax_graph

CASE " $N$ " I Only data parameters exist.

DISP " RAW data."

CASE ELSE

Bad file: DISP CHR\$(12)

DISP "Data file is not recognized, entry aborted."

DISP "...continue."

BEEP

PAUSE

OFF ERROR

GOTO Mistakelineset

END SELECT

1

ENTER @Datapath;Data_id\$

ENTER @Datapath;Datacount

ENTER @Datapath;Hold_size

IF NOT Allocated THEN

IF Datacount $>=1$ AND Hold_size $>=1$ THEN

ALLOCATE Holding_file(Hold_size, 2)

ELSE

ALLOCATE Holding_file( $(9,2)$

END IF

Allocated $=1$

END IF

ENTER @Datapath:Holding_file(")

ASSIGN @Datapath TO *

OFF ERROR

IF Datacount $=0$ THEN Mistakeline

1

ICopy data from Holding_file(") to Basket_file(") 1

MAT Basket_file $=(0$.

IF Datacount > Basketsize THEN IReceiving file too small.

Allocated $=0$

DEALLOCATE Holding_file(")

DISP "DATA FILE overflow, new data discarded. ":

DISP * (continue) *

BEEP

PAUSE

IF Status $\$={ }^{\text {"}} Y$ " THEN 


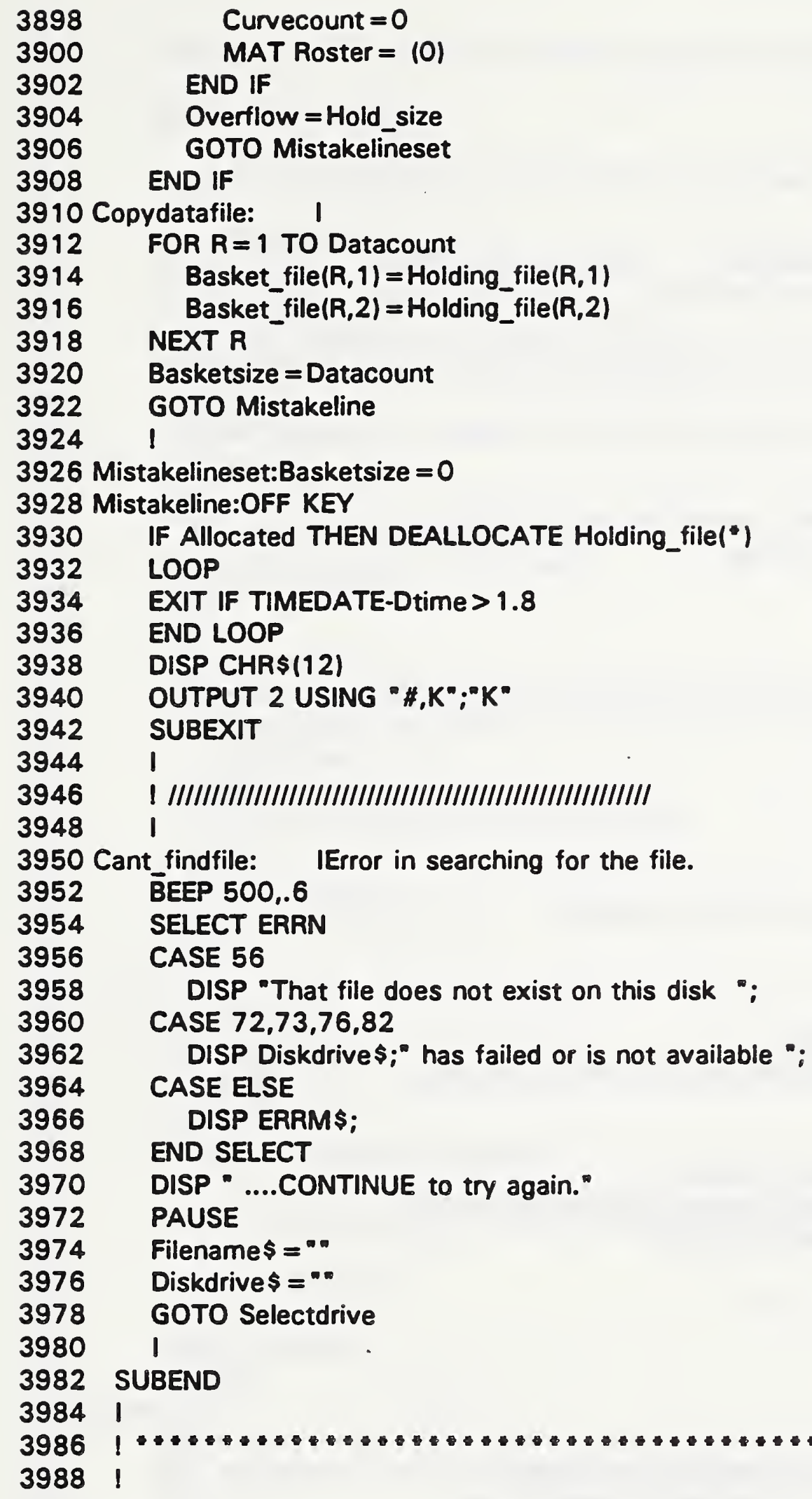




\section{B.5 GAUSS}

146 Gauss:

148

150

152

154

156

158

160

162

164

166

168

170

972

174

176

178

180

182

184

186

188

190

192

194

196

198

200

202

204

I 1

l

CAI 1

END

1

1

!

l

I

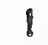

1

I

1

1

1
I RE-STORE "GAUSS:, 1400"

COM /Sys/ Sys_id\$[10]

COM /Sys_msi/Msi_id\$[20]

OUTPUT KBD USING " $K$, " ;" SCRATCH KEYE"

CONTROL KBD, 15;01 sets the color of the soft keys

CONTROL KBD, $2 ; 1$

Intr_prty $=$ ?

CLEAR SCREEN

CALL Gauss

OUTPUT KBD USING "K,\#":"LOAD KEYE" ! restore the typing aid keys PRINT TABXY 19,5$)_{0}^{\circ}$ END of program. So long."

MASS STORAGE IS ", $1400^{*}$

SUB Gauss

OPTION BASE 9

COM /Files/Diskdrive $\$[20], F i l e n a m e \$[14], M$ s_path $\$[500]$

COM /nterrupts/ INTEGER Int?_prty

1 Declare variables

INTEGER Fs,Ptn,Filesize,Datacount,Num

REAL Sigma,Pulse_amp,Time_window,Wv $(32767,21$, Time_var,Temp DIM Data_id\$[40]

I Give information and ask for a value of sigma

PRINT "This program produces an impulse-like, Gaussian waveform."

PRINT "You will need to input a value for sigma."

GOSUB Sigma_in

GOSUB Num_pnts

REDIM WV(Num,2)

I We want to create a gaussian, so starting from the equation

1 for a gaussian $\exp \left(-0.5(x / \text { sigma })^{\wedge \wedge} 2\right)$ we find the value of the

I FWHM. At this point $0.5=\exp \left(-0.5\left((x-\text { mean }) / \text { sigma }^{\wedge}\right)^{\wedge} 2\right)$ so

! [HALF MAX] $=0.693147181=(-0.5) \cdot\left((x-\right.$ mean $) /$ sigma $^{\wedge} \wedge 2$ and

$f(x-$ mean $)=2 \cdot 1.17741 \cdot$ sigma

PRINT "For this sigma, full width-half max $=" 2.35482$ "Sigma

1 Compute the pulse amplitude

GOSUB PIs_amp 
I Ask for the time window in seconds and ask for the filename 1 
318 RETURN

320 Num_err: I

322 BEEP

324 DISP "ERROR IN THE NUMBER OF POINTS, TRY AGAIN"

326 WAIT 1.0

328 Num_pnts: 1

330 Test $\$=$ "

332 ON ERROR GOTO Num_err

334 INPUT "Enter an integer number for the points in the waveform?"

336 IF LEN(Test\$) $<1$ THEN GOTO Num_erp

338 Temp $=$ VAL(Test $\$$ )

340 OFF ERROR

342 IF (Temp $<=0$ ) OR (INT(Temp) $<>$ Temp) THEN GOTO Num_err

344 Num $=$ INT(Temp)

346 RETURN

348 Ampl_err:

350 BEEP

352 DISP "INPUT ERROR, PLEASE TRY AGAIN。"

354 WAIT 9.0

356 Pls_amp:

358 ON ERROR GOTO Ampl_erP

360 inp: Test $\$=" m$

362 INPUT. "Would you like a unit area pulse? $y / n$ (default is y)." Test\$

364 IF LEN(Test $\$$ ) $<1$ THEN Test $\$={ }^{\text {" }} \mathrm{Y}^{\text {" }}$

$366 \quad 1$

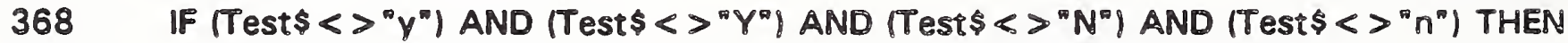

370

372

374

376

378

380

382

384

386

388

390

392

394 Pnt_err:

396 BEEP

398 DISP "INPUT ERROR, PLEASE TRY AGAIN."

400 WAIT 1.0

402 Point place: 1

404 ON ERROR GOTO Pnt_err

406 INPUT "At what point would you like the maximum to occur? "Test\$

408 IF LEN(Test\$) $<1$ THEN GOTO Pnt_err

$410 \quad$ Temp $=$ VAL(Test\$)

412 OFF ERROR

494 IF (Temp $<=0$ ) OR (INT(Temp) $<>$ Temp) THEN GOTO Pnt_err

$416 \quad P$ tn $=$ INT(Temp)

498 RETURN

420 Window err:

422 BEEP

424 DISP "TIME WINDOW INPUT ERROR, PLEASE TRY AGAIN."

426 WAIT 1.0

428 Get wndow: 
INPUT "What is the time window in seconds? "Test\$

IF LEN(Test\$) < 1 THEN GOTO Window_err

CALL Data_check(Test\$)

Time_window $=$ VAL(Test\$)

444

446

448

450

452

454

456 RETURN

IF Time_window $<=0$ THEN

DISP "Time window must be greater than zero."

WAIT 1.0

GOTO Window_err

END IF

OFF ERROR

RETURN

458

460

462

464 Input_amp:

466 ON ERROR GOTO Inp_amp_err

468 Test $\$="$ "

470 INPUT "Enter in the desired pulse amplitude." "Test\$

472 IF LEN(Test\$) < 1 THEN GOTO Inp_amp_error

474 CALL Data_check(Test\$)

476 Pulse_amp $=$ VAL(Test $\$$ )

478 IF Pulse_amp $=0$ THEN GOTO Inp_amp_err

480 OFF ERROOR

482 RETURN

484 SUBEND

$486 \quad$ I

488 II!IIIIII!!I!!

490 SUB Data_to_disk_r(REAL File("),INTEGER Filesize,Datacount,Data_id\$)

492 Data_to_disk_r: I Original: 13 Nov 1984

I This routine will SAVE data files on the disk in RAW data format. I Special features:

1 If the Diskdrive\$ and/or the Filenames are null this routine I will prompt the operator for information. However, if they I are not null it is assumed that the program is supplying the 1 correct information.

!

OPTION BASE 1

COM /Files/Diskdrive $\$\left[20\right.$, Filename $\$[14], M s \_p a t h \$[500]$

CÓM /nterrupts/ INTEGER Intr_prty

INTEGER Local_prty,Diskspace

DIM AC \$[5],Status\$[1]

REAL Dtime

OFF KEY

Local_prty $=$ Intr_prty

Dtime $=0$.

$$
\text { I }
$$

534 Selectdrive: I

ISelect the disk drive for data storage I

IF Diskdrive $\$=$ "NO DISK" THEN Diskdrive $\$="$ "

IF LEN(Diskdrive\$) $>0$ THEN GOTO Choosefilename GRAPHICS OFF 
544 CALL Select_disk

546 IF Diskdrive $\$=$ "NO DISK" THEN GOTO Mistakeline

548 Choosefilename: I

550 IF LEN(Filename \$) $>0$ THEN GOTO Send_to_disk

$552 \quad A C \$=$ "ABORT"

554 CALL Enterfilename(AC\$)

556 IF LEN(Filename $\$$ ) $=0$ THEN GOTO Mistakeline

558 Send to disk: I Create file and save information.

560 ON ERROR GOTO Cant_savedata

562 Diskspace $=$ INT ( Filesize 16.0$) / 256)+2$

564 CREATE BDAT Filename \& \&iskdrive\$,Diskspace, 256

566 Dtime = TIMEDATE

568 DISP " SAVING data in file ";Filename $\$ "$ " on ":Diskdrive $\$$

570 Status $\$=$ " $N$ "

572 ASSIGN @Datapath TO Filename\$\&Diskdrive\$

574 OUTPUT @Datapath;Status\$

576 OUTPUT @Datapath;Data_id\$ 140 chrs description of data

578 OUTPUT @Datapath;Datacount Inumber of xy points

580 OUTPUT @Datapath; Filesize Isize of array

582 OUTPUT @Datapath;File(")

584 ASSIGN @Datapath TO .

586 OFF ERROR

$588 \quad 1$

590 Mistakeline:OFF KEY

592 LOOP

594 EXIT IF TIMEDATE-Dtime $>1.8$

596 END LOOP

598 DISP CHR\$(12)

600 OUTPUT 2 USING ${ }^{*}, K^{\infty}{ }^{\infty}{ }^{\infty} K^{\infty}$

602 SUBEXIT

$604 \quad 1$

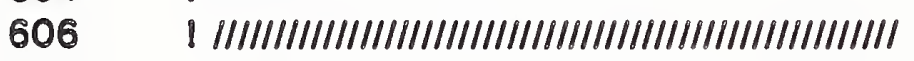

$608 \quad 1$

610 Cant savedata: !

692 BEEP 500.6

614 SELECT ERRN

616 CASE 72,73,76,78,81,82,90,93

618 DISP Diskdrive $\$_{:}^{*}$ has failed or is not available ":

620 DISP *...CONTINUE to try again."

622 PAUSE

624

626

628

630

632

634

636

638

640

642

644

646

648

650

652

Diskdrive $\$=" \infty$

CASE 84,85

DISP " This disk is not initialized":

DISP "....CONTINUE to try again."

PAUSE

Diskdrive $\$=m$

CASE 55,64

DISP " This disk is full, insert new floppy and/or":

DISP " select new drive ...CONTINUE "

PAUSE

Diskdrive $\$=" \varpi$

CASE ELSE

CALL Errortrap

IF LEN(Filename\$) >0 THEN GOTO Send to disk

END SELECT 


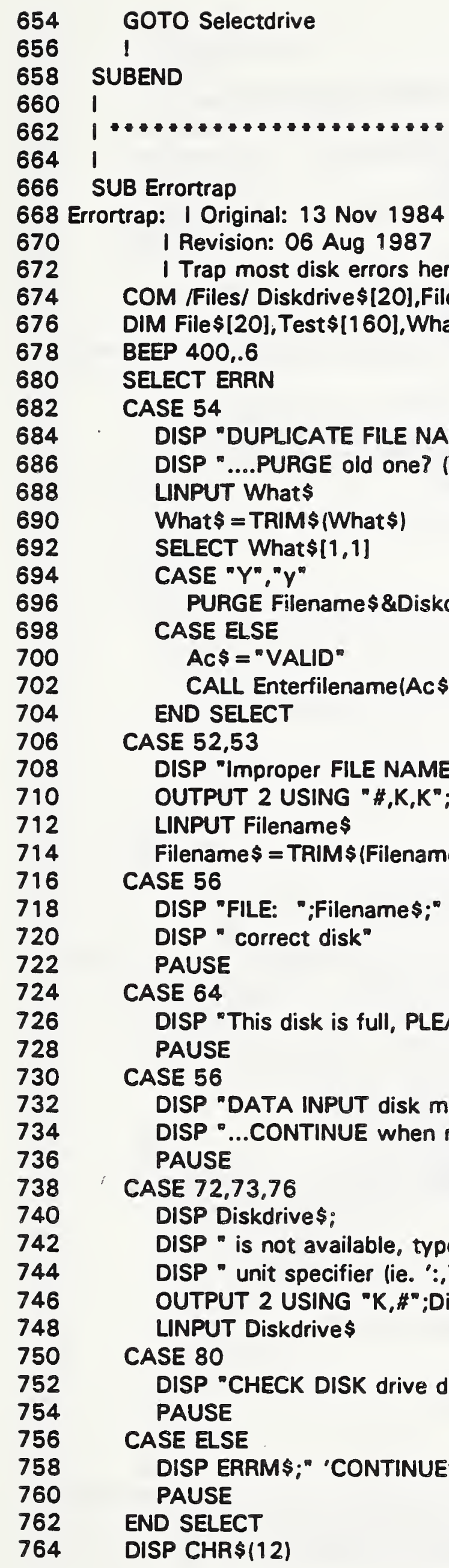




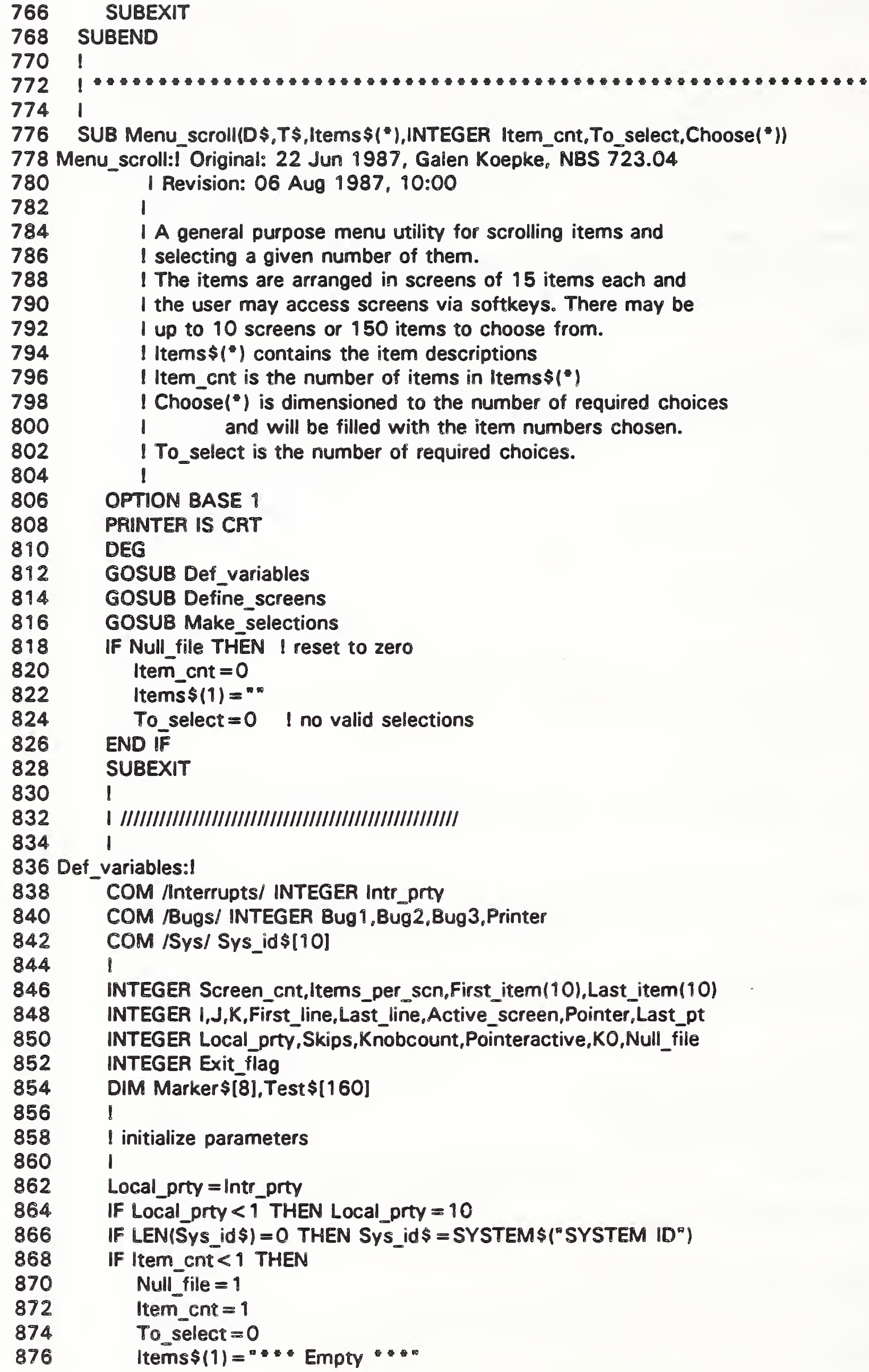




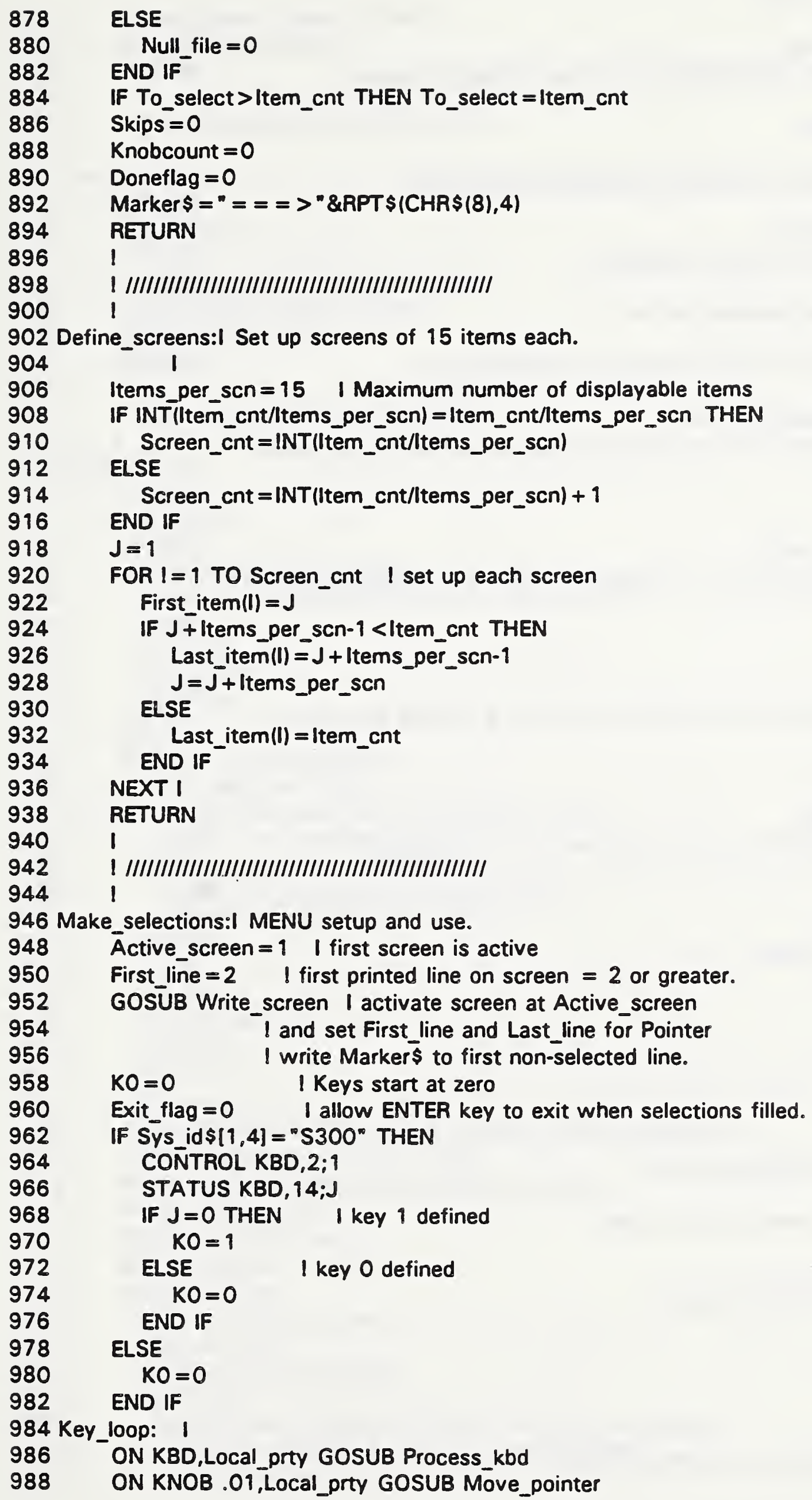

946 Make_selections:I MENU setup and use. 
1012

1014

1016

1018

1020

1022

1024

9026

1028

1030

1032

1034

1036

1038

1040

1042

1044

1046

1048

1050

1052

1054

1056

1058

1060

1062

1064

1064 GOTO Key_loop

1068

1070

1072

1074

1076

1078

1080

1082

1084

1086

1088

1090

1092 I

1094

1096

1098

1100

DISP DS

ELSE

END IF

ELSE

ELSE

END IF

END IF ELSE

OFF KEY KO +1

END IF

ELSE

OFF KEY KO +2

END IF

IF Skips $>0$ THEN ELSE

OFF KEY $\mathrm{KO}+3$

END IF

ELSE

OFF KEY KO +4

END IF

MAT Choose $=101$

To_select $=0$

xit_line:OFF KEY

OFF KNOB

OFF KBD

PRINT CHR\$ 1928):

RETURN

!

1

I

OFF KBD

OFF KNOB

OFF KEY

IF Skips < To_select THEN

IF To_select $>1$ THEN

$$
\text { Test } \$=\text { " Select "\&VAL\$(Skips + 1)\&" of "\&VAL\$(To_select) }
$$

Test $\$=$ "Select"

ON KEY KO LABEL Test\$_Local_prty GOSUB Select_item

IF To_select>O THEN

DISP " Selection process complete ..."

DISP " Menu for information only ..."

ON KEY KO LABEL "Accept" "Local_prty GOTO Exit_line

If Active_screen $<$ Screen_cnt THEN

ON KEY KO + 8 LABEL " Next Screen",Local_prty GOSUB Next_screen

IF Active screen $>1$ THEN

ON KEY KO + 2 LABEL "Last Screen" „Local „prty GOSUB Last_screen

ON KEY KO + 3 LABEL "Reset Select" "Local_prty GOSUB Select_reset

IF TO_ select $>0$ THEN

ON KEY KO + 4 LABEL " Abort " ¿Local _prty GOTO Escape_line

IF Exit flag THEN Exit_line

OUTPUT KBD;CHR\$ (255)\&CHR\$(75):

I everything cleared, now go back to work.

I | IIIIIIIIIIIIIIIIIIIIIIIIIIIIIIIIIIIIIIIIIIIIIIIII

IF Active_screen = Screen_cnt THEN RETURN 
Active_screen $=$ Active_screen +1

1104

1106

1108

1110

1112

1114 Last

1116

1118

1120

1122

1124

1126

1128

1130

1132

1134

1136 Select_item:!

1138

1140

1142

1144

1146

1148

1150

1152

1154

1156

1158

1160

1162

1164

1166

1168

1170

1172

1174

1176

1178

1180

1182

1184

9186

1188

1190

1192 GOSUB Write_screen

RETURN

l

! IIIIIIIIIIIIIIIIIIIIIIIIIIIIIIIIIIIIIIIIIIIIIIIIIII!

!

OFF KBD

OFF KNOB

OFF KEY

IF Active_screen $=1$ THEN RETURN

Active_screen =Active_screen-1

GOSUB Write_screen

RETURN

I

! IIIIIIIIIIIIIIIIIIIIIIIIIIIIIIIIIIIIIIIIIIIIIIIII

!

OFF KBD

OFF KNOB

OFF KEY

IF NOT Pointeractive THEN

DISP "NO additional selections for this screen."

BEEP

WAIT 2

DISP CHR\$(12):

RETURN

END IF

IF Skips $=$ To_select THEN

IF To_select $=0$ THEN

DISP "This menu is for information only" :

DISP " no selection allowed."

ELSE

DISP "All selections have been filled,":

DISP " "Select Reset' to repeat."

END.IF

BEEP

WAIT 2

DISP CHR\$(12):

RETURN

END IF

Skips $=$ Skips +1

Choose(Skips) = First_item(Active_screen) + Pointer-First_line

PRINT CHR\$1929): I inverse video

PRINT TABXY(10,Pointer);items $\$$ (Choose(Skips))

PRINT CHR\$ (128);

1194

1196

1198

1200

1202

PRINT TABXY(1,Pointer):

SELECT Pointer

CASE First_line

GOSUB Point_forward

CASE Last line

GOSUB Point_backward

1208

1210

1212

CASE ELSE

1 move forward unless it requires wrapping to beginning.

IF Skips-1 >0 THEN ! check for selected items. $I=$ Pointer-First_line 


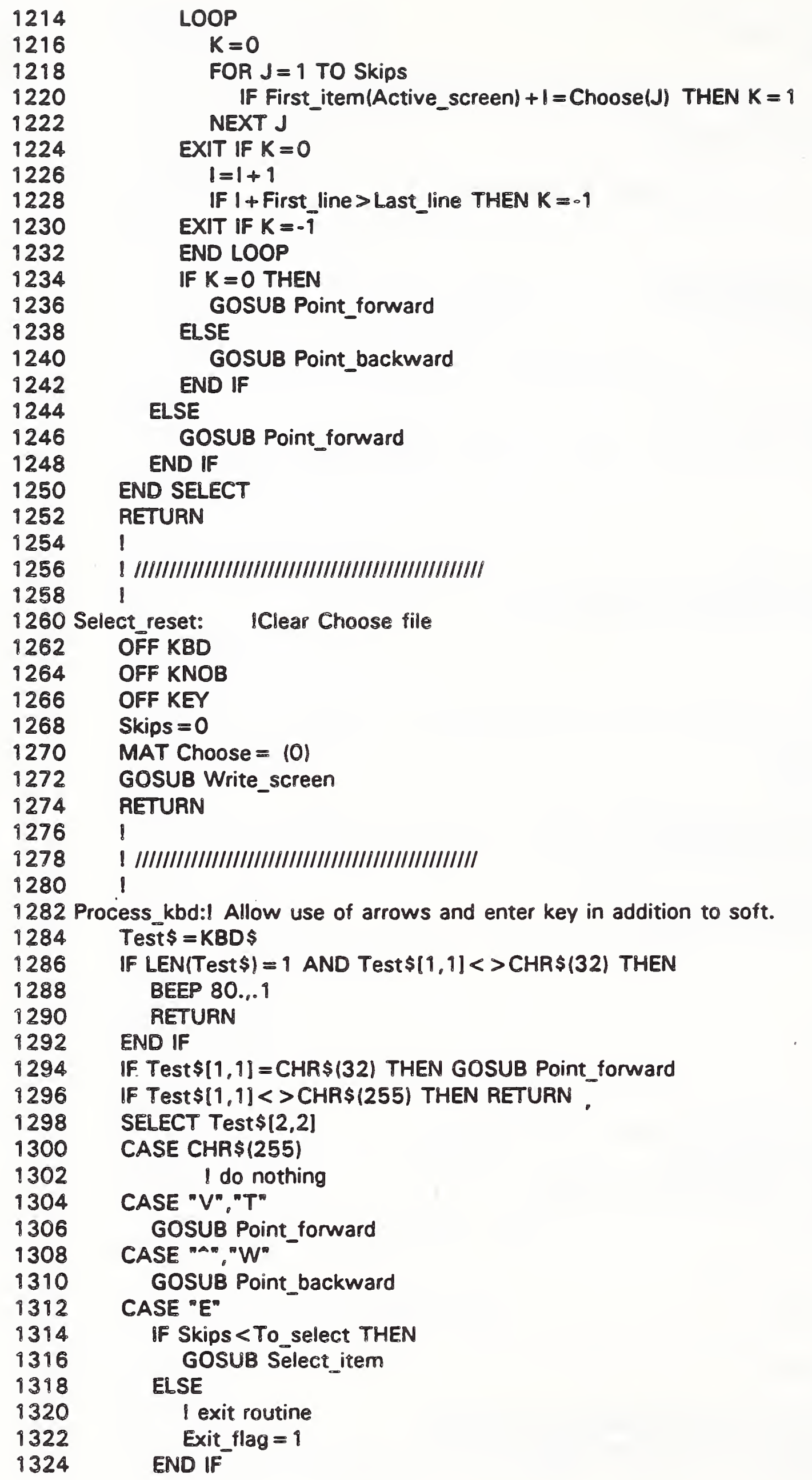


1342 Point forward: Knobcount $=5$

1344 ḠOSUB Move_pointer

1346 RETURN

1348 Point backward: Knobcount $=-5$

1350 ḠOSUB Move_pointer

1352

1354

1356

1358

1360 Jog_pointer:! Move the selection pointer on the active screen.

1362

1364

1366

1368

1370

1372

1374

1376

1378

1380

1382

1384

1386 Move pointer:! Control pointer to avoid re-selection of items

1388

1390

RETURN

!

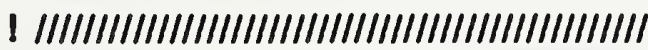

1

I without regard to selected values

IF Knobcount >0 THEN ! Move forward

Pointer $=$ Pointer +1

ELSE

I Move backward

END IF

Pointer $=$ Pointer -1

IF Pointer < First_line THEN Pointer $=$ Last_line

IF Pointer $>$ Last_line THEN Pointer $=$ First line

RETURN

1

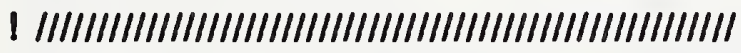

1

IF NOT Pointeractive THEN RETURN \& No selections to be made.

1392

1394

1396

1398

1400

1402

1404

1406

1408

1410

1412

1414

1416

1418

1420

1422

1424

1426

1428

1430

1432

1434

1436 Knobcount $=$ Knobcount + KNOBX + KNOBY

IF ABS(Knobcount) $<4$ THEN RETURN

Last_pt $=$ Pointer

GOSUB Jog_pointer

IF Skips $>0$ THEN

LOOP

$\mathrm{J}=$ Pointer-First line

FOR $I=1$ TO Sḱips

IF First item(Active_screen) $+J=$ Choose(I) THEN $J=999$

NEXT 1

IF $J=999$ AND Pointer $=$ Last_pt THEN Pointeractive $=0$

EXIT IF Pointeractive $=0$

IF $J=999$ THEN GOSUB Jog_pointer

EXIT IF $\mathrm{J}<>999$

END LOOP

END IF

Knobcount $=0$

OUTPUT KBD;CHR\$(255)\&CHR\$(84); I Bring screen home

IF Last_pt $=$ Last_line THEN PRINT CHR\$(132);

PRINT " ":

IF Pointeractive THEN ! Pointer active

If Pointer $=$ Last_line THEN

PRINT CHR\$ (132):

ELSE 
1438

1440

1442

1444

1446

1448

1450

1452

1454 Write_screen:I Write the screen pointed to by Active_screen

1456

1458

1460

1462

1464

1466

1468

1470

1472

1474

1476

1478

1480

1482

1484

1486

1488

1490

1492

1494

1496

1498

1500

1502

1504

1506

1508

1510

1512

9514

1516

1518

1520

1522

1524

1526

1528

1530

1532

1534

1536

1538

$\$ 540$

1542

1544

1546

1548

PRINT CHR\$(128):

END IF

END IF

PRINT TABXY(1,Pointer);Marker\$;CHR\$(128);

RETURN

1

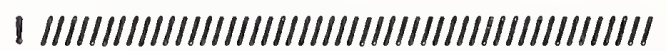
1

I home and clear screen

OUTPUT KBD;CHR\$(255)\&CHR\$(84)\&CHR\$(255)\&CHR\$(75):

Knobcount $=K N O B X+$ KNOBY $\mid$ Clear knob and keyboard

Knobcount $=0$

Test $\$$ KBD\$

Test $\$={ }^{\text {क }}$

l

PRINT TABXY(1,First_line-1);CHR\$(132):" Item \#| Screen \#";

PRINT USING " \#,2D,3̈A,2D,3A":Active_screen," of ";Screen_cnt; 1 "

PRINT T\$;RPT \$ ${ }^{\circ}{ }^{\circ}, 52$-LEN(T \$);:CHR\$(128);

$J \equiv 0$

REPEAT

If $J=$ Last item(Active_screen)-First_item(Active_screen) THEN PRINT C̈HR\$ (132):

PRINT TABXY(1,First_line + J);RPT $\$\left({ }^{*} ", 80\right)$

ELSE

PRINT CHR\$1928):

END IF

PRINT TABXY(5, First_line + J);

PRINT USING " $30, A_{\diamond} \#^{\prime \prime}$; First item(Active_screen) $+J_{0}{ }^{\circ} 1 "$

IF Skips $>0$ THEN I make this line inverse video

FOR $I=1$ TO Skips

IF First item(Active_screen) $+\mathfrak{J}=$ Choose(I) THEN PRINT CHR\$ $192 \overline{9})$;

END IF

NEXT I

END IF $J=J+1$

UNTIL $d>=$ (Last_item(Active_screen)-First_item(Active_screen) +1 )

Last_line $=$ Last_item (Active_screen)-First_item(Active_screen)

Last_line = Last_line + First_line

1

I set marker to first non-selected item.

1

Pointeractive $=0$

IF To_select $>0$ THEN Pointeractive $=1$

IF Skips $>0$ AND Pointeractive $=1$ THEN $\mid$ find first non-selected item $J=0$

LOOP

Pointer $=$ First line $+J$

FOR $I=1$ TO Skips

IF First_item(Active_screen) $+d=$ Choose(I) THEN Pointer $=0$ NEXT I

EXIT IF Pointer $<>0$

$J=J+1$

IF First_line $+d>$ Last line THEN

PRINT TABXY(10,First_line $+J)$ iltems $\$$ (First_item(Active_screen) $+J)$ 
1558

1560

1562

1564

1566

1568

1570

1572

1574

1576

1578

1580

1582

1584

1586

1588

1590

1592

1594

1598

1600

1602

1604

1606

1608

1610

1612

1614

1616

1618

1620

1622

1624

1626

1628

1630

1632

1634

1636

1638

1640

1642

1644

1646

1648

1650

1652

1654

1656

1658

1660
Pointeractive $=0$

END IF

Pointer $=$ First_line

EXIT IF Pointer $<>0$

ELSE

END LOOP

Pointer $=$ First_line

END IF

IF Pointeractive THEN

IF Pointer $=$ Last_line THEN PRINT CHR\$(132);

ELSE

PRINT CHR\$(128);

END IF

END IF

PRINT TABXY(1,Pointer);Marker\$;CHR\$(128);

RETURN

SUBEND

1

1

SUB File_menu(Mask\$,Ftype\$,Fls\$("),INTEGER Fls_cnt,Dir_on,Prt_on)

e_menu: !

I Original: 29 Jun 1987, G. Koepke

I Revision: 06 Aug 1987, 10:00

OPTION BASE 1

DEG

COM /Sys/ Sys_id $\$[10]$

COM /Files/ Diskdrive $\$[20], F i l e n a m e \$[14], M s \_p a t h \$[500]$

COM Anterrupts/ INTEGER Intr_prty

DIM Directory $\$(150)[80], B d \$(150)[71]$

DIM D \$[80],T\$[52],Ids\$[40],Stat\$[1]

INTEGER Bd_cnt,File_cnt,l,C_cnt,CO(1),Format_error

IF FIs_cnt $>0^{-}$THEN ALLLOCATE INTEGER Choose(Fls_cnt)

1

I Catalog the disk specified

1

ON ERROR GOTO Cat_errors

DISP " Reading the Directory ..."

MASS STORAGE IS Diskdrive $\$$

CAT TO Directory \$("):NO HEADER,COUNT File_cnt OFF ERROR

1

I set up array of legal file names.

1

Bd_cnt $=0$

FO $\bar{R} !=1$ TO File_cnt

IF Directory $\$(1)(32,36]=$ Ftype $\$$ THEN I Ftype $\$=$ "BDAT "

I Ftype $\$=$ "PROG "

IF LEN(Mask\$) >0 THEN I Test for mask\$

IF Directory $\$(1)[1$, LEN(Mask $\$)]=$ Mask $\$$ THEN

Bd_cnt $=$ Bd_cnt +1

$B d \bar{s}\left(B d \_c n t\right)=$ Directory $\$(1)[1 ; 10]$

END IF

ELSE

$B d \_c n t=B d \_c n t+1$ 
1746

1748

1750

1752

1754

1756

1758

1760

1762

1764

9766 Read_data_id: ! This routine expects to see Ids $\$$ from

1768

1770

1772

END IF

$B d \$\left(B d \_c n t\right)=$ Directory $\$(1)(1 ; 10)$

END IF

NEXT I

I

I set up file menu

1

$D \$=$ "Select "\&VAL\$(FIs_ent)\&" file names for data entry."

$T \$=$ "List of "\&Ftype $\$$ " files on "\&Diskdrive $\$$

IF LEN(Mask\$) $>0$ THEN

$$
T \$=T \$ \& " \text { mask }=\text { "\&Mask\$ }
$$

END IF

IF Bd_Cnt $>0$ THEN

IF Dir_on $>0$ THEN GOSUB Read_data_id

IF Prt_on THEN

GŌSUB List_directory

ELSE

$C_{-} \mathrm{cnt}=\mathrm{Fls}$ c cnt

DISP CHR\$ (12)

IF FIs_cnt $>0$ THEN

ELSE

CALL Menu_scroll(D\$,T\$,Bd\$("),Bd_cnt,C_cnt,Choose("))

CALL Menu_scroll(D\$, T\$,Bd\$("),Bd_Cnt,C_Cnt,CO(*)1)

END IF

1

I transfer file names to FIs\$(").

1

IF $C_{-}$cnt $=0$ THEN $\mid$ selection process aborted MAT FIs $\left.\$=(")^{*}\right)$

ELSE

MAT SORT Choosel")

FOR I $=1$ TO C c cnt

Fls $\$(1)=$ Bd $\$($ Choose $(1))[1 ; 10]$ NEXT I

END IF

END IF

\section{ELSE}

DISP * This directory contains no BDAT files ..."

WAIT 2.5

END IF

DISP CHR\$(12)

SUBEXIT

Cat_errors:!

DISP "ERROR ... ";ERRM\$

BEEP

PAUSE

C c c nt $=0$

MAT FIS $\$=(" n)$

SUBEXIT

1

! IIIIIIIIIIIIIIIIIIIIIIIIIIIIIIIIIIIIIIIIIIIIIIIIIII

$$
1
$$

1 GRAPH DATA raw data files.

DISP "Reading file contents ..."

FOR $I=1$ TO Bd_cnt $\mid$ each BDAT file 


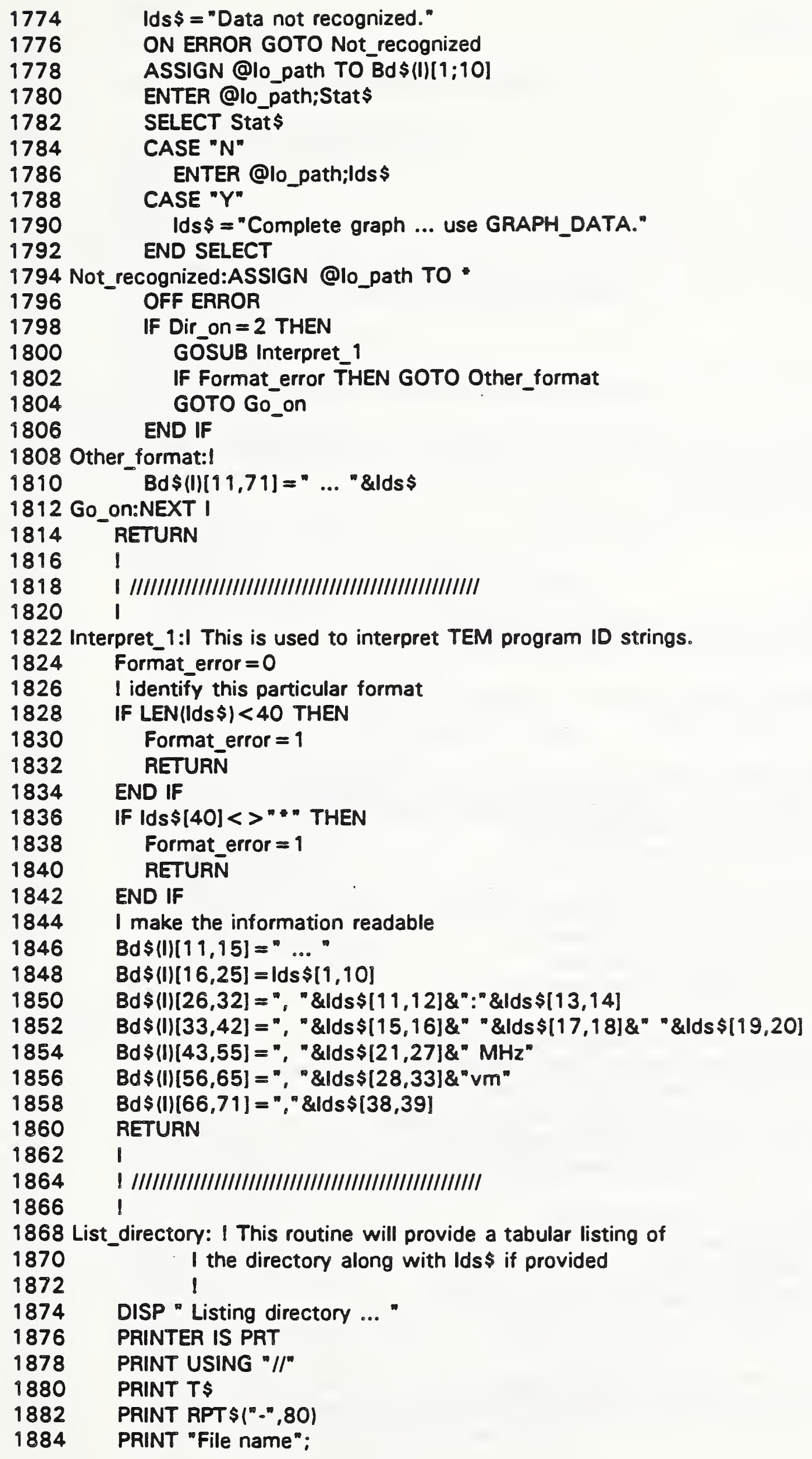


IF Dir_on THEN

PRINT * ... contents"

ELSE

PRINT

END IF

PRINT RPT $\$\left({ }^{\circ}=", 80\right)$

FOR $l=1$ TO Bd_cnt PRINT Bd\$(I)

NEXT :

PRINT RPT $\$ 1 "=801$

PRINT

PRINTER IS CRT

RETURN

SUBEND

1

1

SUB Load_disk_data(Basket_file("),INTEGER Basketsize,Data_id\$,INTEGER Flg)

Load_disk_data: I Original: 13 Nov 1984

I Revision: 02 Dec 9987

IThis routine will enter data files from the disk OPTION BASE 1

!

COM /Sys/ Sys id\$

COM /History/ Status\$[1],Time_orgn\$[8],Date_orgn\$[19]

COM /History/ Time_chng\$[8],Date_chng\$[11],Description\$[160] 1

COM /Labels/ Labels $\$(30)[60]$,INTEGER Lbl_count,REAL Lbl_addr(30,6) ILbl_addr: $x, y$, pen, size, LDIR, LORG

COM /Data_param/ INTEGER Datacount,Filesize,Curvecount,Roster $(17,4)$

COM /Data_param/ REAL Sym_size,Symbol\$(17)[2],Curve_id $\$(17)[40]$

COM /Data_param/ REAL Xmin_data,Xmax_data 1

COM /Data_param/ REAL Ymin_data,Ymax_data

!Roster: Curve\#, Start Addr in File(*), Datacount, and PEN

!Symbol\$(i) $={ }^{* \mathrm{~N}}$ or " $Y^{\mathrm{F}} \Rightarrow>$ no symbol, connect pts

ISYmbol $\$(i)={ }^{*} \mathrm{Y}^{*}=>$ symbol, connect pts

ISymbol\$(i) $={ }^{*} \Rightarrow \mathrm{N}^{*} \Rightarrow$ symbol, do not connect pts 1

COM /Background/ Graphtype \$[12],Margins\$(2)[10],Papersize \$[1]

COM /Background/ REAL Pen_speed,INTEGER Backgnd_pen,Auto_time

COM /Background/ INTEGER Auto_file,REAL $X_{-}$cross_ $y$, $Y$ _cross_ $x$

COM /Background/ Xgrid_tick\$[4],INTEGER Xmajor,Xminor

COM /Background/ Ygrid_tick\$[4],INTEGER Ymajor,Yminor

COM /Background/ REAL Xmin_graph,Xmax_graph,Ymin_graph,Ymax_graph

1

COM /Bugs/ INTEGER Bug 1,Bug2,Bug3,Printer

COM /nterrupts/ INTEGER Intr_prty

COM /Enlarge_file/ INTEGER Overflow

COM /Files/ Diskdrive $\$[20]$,Filename $\$[14]$,Ms_path $\$[500]$

COM /Data_stuff/ INTEGER Number,REAL Delta_x

INTEGER R,Hold_size,Local_prty,Allocated,FIs_cnt

DIM AC $\$[5], T e m p f i l e \$(10)$, Mask $\$[10], F$ type $\$[5], F I s \$(1)[14]$

REAL Dtime 


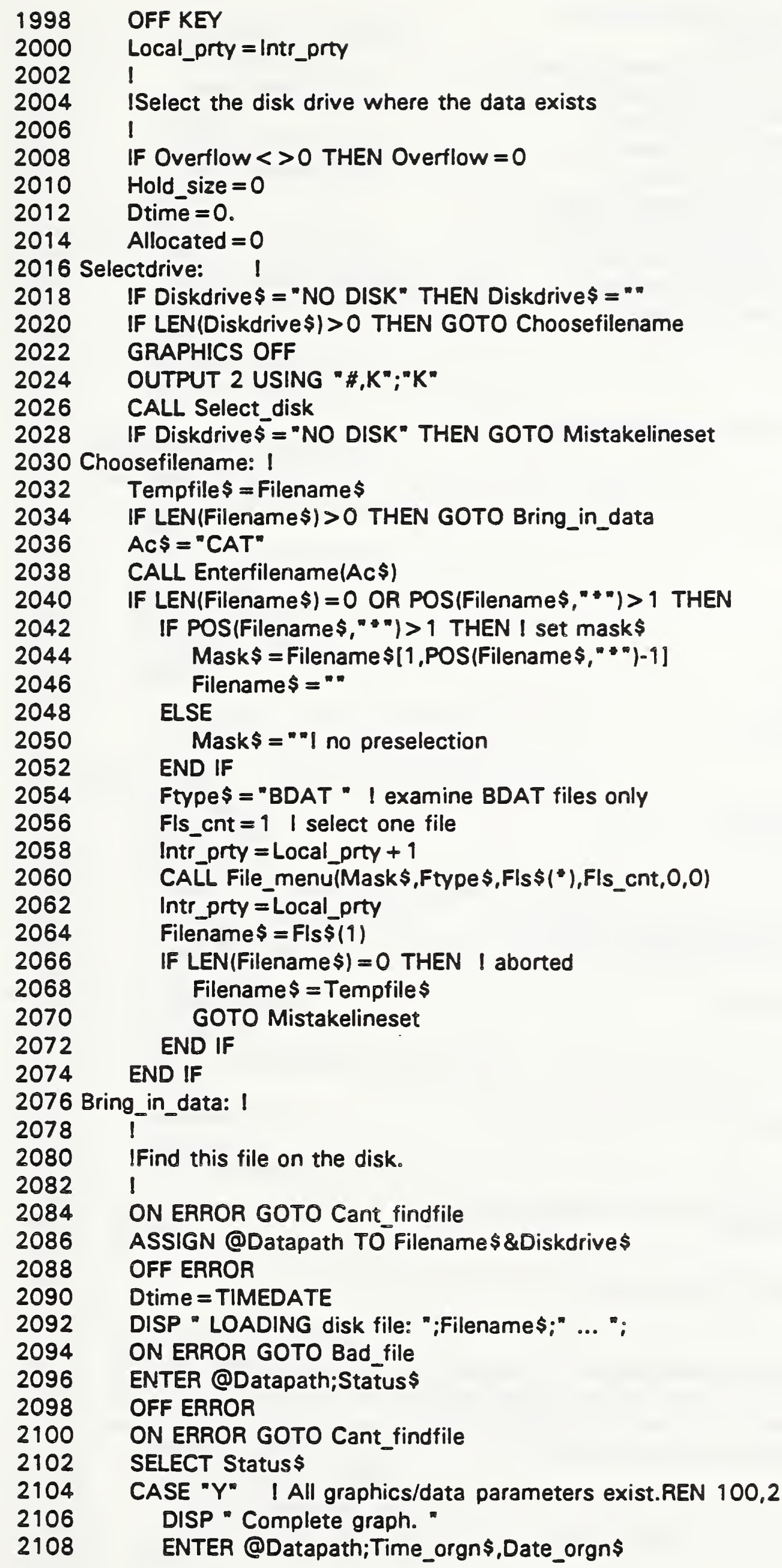


ENTER @Datapath;Time_chng\$,Date_chng\$ ENTER @Datapath;Description\$

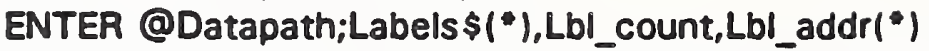

2116

2138 Bad_file: DISP CHR\$(12)

2140

2142

2144

2146

2148

2150

2952

2154

2956

2158

2160

2162

2164

2166

2168

2170

2172

2174

2176

2178

2180

2182

2184

2186

2188

2190

2192

2194

2196

2998

2200

DISP "Data file is not recognized, entry aborted.": DISP " ...continue."

BEEP

PAUSE

OFF ERROR

GOTO Mistakelineset

END SELECT

1

ENTER @Datapath;Data_id\$

IF FIg THEN

ENTER @Datapath;Deita_x

ENTER @Datapath;Datacount

Hold_size $=$ Datacount

ELSE

ENTER @Datapath;Datacount

ENTER @Datapath;Hold_size

END IF

IF NOT Allocated THEN

IF Datacount $>=1$ AND Hold_size $>=1$ THEN

ALLOCATE Holding_file(Hold_size,2)

ELSE

ALLOCATE Holding_file $(1,2)$

END IF

Allocated $=1$

END IF

ENTER @Datapath;Holding_file(")

ASSIGN @Datapath TO .

OFF ERROR

IF NOT Fig THEN Delta_ $x=$ Holding_file $(2,1)$-Holding_file $(1,1)$

IF Datacount $=0$ THEN Mistakeline

I

ICopy data from Holding_file(") to Basket_file(")

I

MAT Basket_file $=10.1$

IF Datacount $>$ Basketsize THEN IReceiving file too small.

Allocated $=0$

DEALLOCATE Holding_file(")

DISP " DATA FILE overflow, new data discarded. ":

DISP * (continue) "

BEEP

PAUSE 
END IF MAT Roster $=(0)$

Overflow $=$ Hold_size

END IF

GOTO Mistakelineset

Copydatafile:

FOR $R=1$ TO Datacount

Basket_file $(R, 1)=$ Holding_file $(R, 1)$

Basket_file $(R, 2)=$ Holding_file $(R, 2)$

\section{NEXT R}

Basketsize $=$ Datacount

GOTO Mistakeline

2252 Mistakelineset: Datacount $=0$

2254 Mistakeline:OFF KEY

2256

2258

2260

2262

2264

2266

2268

2270

2272

2274

2276

2278

2280

2282

2284

2286

2288

2290

2292

2294

2296

2298

2300

2302

2304

2306

2308

2310

2312

2314

2316

2318

2320

2322

2324

2326

2328

2330

2332

IF Allocated THEN DEALLOCATE Holding_file( $\left.{ }^{*}\right)$

LOOP

EXIT IF TIMEDATE-Dtime $>1.8$

END LOOP

DISP CHR\$(12)

OUTPUT 2 USING "\#,K";"K"

SUBEXIT

I

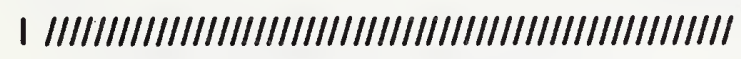

1

Cant_findfile: IError in searching for the file.

BEEP 500,.6

SELECT ERRN

CASE 56

DISP "That file does not exist on this disk ";

CASE $72,73,76,82$

DISP Diskdrive $\$ ; "$ has failed or is not available ":

CASE ELSE

DISP ERRM\$;

END SELECT

DISP " ....CONTINUE to try again."

PAUSE

Filename $\$=" *$

Diskdrive $\$=" \|$

GOTO Selectdrive

1

SUBEND

1

1

SUB Data_check(Test\$)

IThe following checks for a lower case " $e$ " in an input number and,

lif it exists, converts it to a number with an upper case " $E$ ". The

Icomputer will recognize only upper case input otherwise.

INTEGER Temp

IF POS(Test\$," $\left.e^{n}\right)$ THEN

Temp $=$ POS $\left(\right.$ Test $\left.\$, "{ }^{n}\right)$

Test\$[Temp] $={ }^{"}{ }^{\infty}$ " \& Test\$ $[$ Temp +1, LEN(Test\$) $]$

END IF 
lend of conversion.

SUBEXIT

2338 SUBEND

2340 SUB Select_disk

2342 Select_disk: I Original: 13 Nov 1984

2344

I Revision: 02 Dec 1987

2348

2350

2352

2354

2356

2358

2360

2362

2364

2366

2368

2370

2372

2374

2376

2378

2380

2382

2384

2386

2388

2390

2392

2394

2396

2398

2400

2402

2404

2406

2408

2410

2412

2414

OPTION BASE 1

COM /Files/ Diskdrive \$[20],Filename \$ 1 4 4],Ms_path $\$[500]$

COM Interrupts/ INTEGER Intr_prty

COM /Sys_msi/ Msi_id\$

COM /Sys/ Sys_ids

INTEGER Local_prty,Dd „PR,Choose(1)

DIM Disc\$(30)[60], Title \$[40],Displ\$[60]

Local_prty $=$ int?_prty

OFF KEY

1

1 Define the disk drives available for this system, reserve the

I first characters for the drive address and the characters after

I the - for a description of the drive.

1

1 Example:

I Dise $\$(9)={ }^{\circ}: 700,0,0$ HP 9133 H HARD disk, volume $0 .{ }^{\circ}$

!

Displ\$ $=$ " SELECT DISK DRIVE ... Abort will cancel."

Title $\$=$ "Available disk drives for this system. "

$P t=1 \quad I$ allow only one select

!

IF Diskdrive $\$[1,1]<>0^{*}$ THEN Diskdrive $\$=$ "

IF Msi_id $\$ 1,1]<>{ }^{* *}$ THEN Msi_id $\$=$ SYSTEM $\$\left(" M S I^{*}\right)$

IF Msi id $\$[1,1]<>"$ : THEN I Must be HFS subdirectory

Ms_path $\$=$ Msi_id $\$\left[1, P O S\left(M s i\right.\right.$ id $\${ }_{0}{ }^{m}: " \%$ \% 1 I strip off subdirs

IF Ms_path $\$$ [LEN (Ms_path $\$ 1: 1]<>\%$ THEN Ms_path $\$=M s \_p a t h \$ \& " / "$

Msi_id $\$=$ Msi_id \$ [POS(Msi_id\$," :"), LEN(Msi_id\$)]

END IF

Diskdrive $\$=$ TRIM $\$$ (Diskdrive $\$$ )

Msi_ids = TRIM\$(Msi_id\$)

IF LEN(Diskdrive $\$$ ) $>\overline{0}$ AND LEN(Msi id $\$$ ) $>0$ THEN

Disc $\$(1)=$ Diskdrive \$\&RT $\$\left({ }^{\circ}\right.$ ". 17-LEN(Diskdrive $\left.\$ 1\right)$

Disc $\$(1)=$ Disc $\$(1) \& "$ - Last selected disk drive."

$\mathrm{Dd}=q^{\prime}$

IF Diskdrive $\$<>$ Msi_id\$ THEN

Disc\$(2)=Msi_id \$\&RPT \$ $1^{\circ}$ "17-LEN(Msi_id \$11

Disc $\$(2)=\operatorname{Disc} \$(2) \& "$ - Start-up mass storage unit specifier."

IF LEN(MSi_id\$) $>0$ THEN

Disc $\$(1)^{-}=$Msi id \$\&RPT $\$\left(1^{\circ}\right.$ * 97-LEN(MSi_id \$)

Disc $\$(1)=$ Disc $\$(1) \&^{*}$. Start-up mass storage unit specifier. ${ }^{\text {" }}$

$$
\mathrm{Dd}=9
$$

ELSE

$D d=0$

2444

END IF 
2530 Enterfilename: I Original: 13 Nov 1984

Disc $\$(D d+1)=":, 702,0$ - HP 9122 dual microfloppy left drive"

Disc $\$(D d+2)=":, 702,1 \quad$ - HP 9122 dual microfloppy right drive"

Disc $\$(D d+3)=":, 703,0$ - HP 9125 single 5.25 floppy drive"

Disc\$(Dd + 4) =":,1400 - HP 9133H hard disk volume $1 "$ I

$D d=D d+4 \quad I$ add the number of drive specifiers above !

IF Sys_id $\$ 1,4]<>$ "S300" THEN

Disc $\$(D d+1)=":, 4,1 \quad$ - LEFT internal series $200^{n}$

Disc $\$(D d+2)=": 4,0 \quad-$ RIGHT internal series $200^{\circ}$

END IF

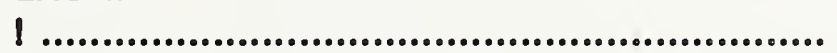

1

CALL Menu scroll(Displ\$,Title\$,Disc\$("),Dd,Pt,Choose("))

IF $P t=0$ THEN

ELSE

$\operatorname{Dd}=$ POS (Disc $\$($ Choose $(P t)), "=")-1 \quad \mid$ find -

If Dd>5 THEN I valid msus

ELSE

DISP " ERROR in reading MSUS from string, - chr not found."

BEEP

CALL Pause_key_on

END IF

Diskdrive\$ $=$ "NO DISK"

END IF

SUBEXIT

SUBEND

1

1

SUB Enterfilename(Ac\$)

I Revision: 10 Dec 1990 includes HFS directories OPTION BASE 1

COM /Files/ Diskdrive $\$$ [20], Filename $\$$ [14],Ms_path $\$[500]$

COM /interrupts/ INTEGER Intr_prty

INTEGER I,Ascii_num,Maskflag,Namelength

DIM Test $\$[256]$,Hfs_temp\$[161]

Namelength $=10$

IF LEN(Ms_path\$) >0 THEN OUTPUT KBD USING "K, \#" ;" \#" \&Ms_path $\$ \&^{n} H^{n}$

DISP " ENTER HFS directory PATH (no file)";

IF AC\$ $<>$ "PATH" THEN

DISP ", ENTER / for HFS ROOT or nUll for LIF...";

END IF

LINPUT Hfs_temp\$ 
His_temp $\$=$ TRIM $\$$ (Hfs_temp $\$$ )

IF LEN(Hfs_temp\$ $1>0$ THEN

IF LEN(Hfs_temp\$) $>1$ AND Hfs_temp\$[LEN(Hfs_temp\$); 1$]<>" I^{\circ}$ THEN Hfs_temp $\$=H f s \_t e m p \$ \&{ }^{m} /{ }^{m}$

END IF

DISP " ENTER the FILE NAME ...":

SELECT AC $\$$

CASE "CAT"

DISP "(ENTER CAT mask " Or ENTER NUll to CAT)":

\section{CASE "ABORT"}

DISP "(ENTER null to ABORT) ";

CASE "VALID"

DISP "(must be a VALID namel) ":

END SELECT

LINPUT Test\$

Test $=$ TRIM $\$($ Test $\$)$

IF LEN(Test $\$$ ) $=0$ AND AC $\$=$ "VALID" THEN GOTO Enterfilename

IF LEN(Test $\$$ ) $=0$ THEN Abortline

IF LEN(Test\$) > Namelength THEN

BEEP

DISP "ERROR in NAME ENTRY - max ";Namelength;" chars, you have ";

DISP LEN(TeSt\$):"

WAIT 9.8

OUTPUT 2 USING "K,\#";"\#" \&Test\$\&"H"

GOTO Efn

END IF

If POS $(T e s t \$, " *)>1$ THEN

Test $\$=$ Test $\$\left[1\right.$, POS(Test\$, ${ }^{*} "$ ") -1$]$

Maskflag $=1$

ELSE

Maskflag $=0$

END IF

FOR $1=1$ TO LEN(Test\$)

Ascii_num $=$ NUM(Test $\$$ (I))

SELECTT Ascii_nUm

CASE 65 TO $90,95,97$ TO 122,48 TO 57

IAllowed characters

CASE ELSE

BEEP

DISP "ERROR in NAME ENTRY-ILLEGAL CHARACTERS, TRY AGAIN." WAIT 9.8

OUTPUT 2 USING ${ }^{n} K_{0} \#^{n}:^{n} \#^{n}$ \&Test $\$ \&^{n} H^{n}$

GOTO EFn

END SELECT

NEXT I

IF Maskflag THEN

Filename $\$=$ Test $\$ \&^{m * n}$ 
Ms_path $\$=H f s \_t e m p \$$

SUBEEXIT

2680 Abortline:Filename $\$="$ "

2682

2684

IF AC $\$=$ "CAT" THEN Ms_path $\$=H f s$ temp $\$$

SUBEXIT

2686 SUBEND

26881

2690

2692 
102

104 SUB User_sub(REAL Wave("I,INTEGER D_volume,D_count)

1061

108 Histogram only:!

110 OPTIONN BASE 1

112 RAD

114 REAL Vmin,Vmax,V_last,Volt_z,Volt_100,V_first,Vptp

116 REAL Histogram(163̄84,2)

118 INTEGER No_o_bins, His_zero_lev

120. INTEGER His_100_lev, Maxpoint

122 REAL Delta_v,Delta_v_prc,Ov,Undr

124

126 Dateline: I 1

128

INTEGER Indx,Min_bin,Half_bin,l,Level,Zipo,Hundred,Done,Auto,Pnts DIM Ch\$[1],X_units\$[151,Y units $\$ 195]$

ALLOCATE Hist_ary(16384)

Pnts $=$ D_count

INPUT "What are the units of the $x$ axis?" $X$ units $\$$

INPUT "What are the units of the $y$ axis?" $Y$ units $\$$

GOSUB Mak_histogram

REDIM Histogram(No_o_bins, 2), Wave(No_o_bins, 2)

MAT Wave $=$ Histogram

D_count $=$ No_o_bins

\section{DEALLOCATÉ Hist_aryl")}

SUBEXIT

1

DISP "Calculating the histogram, please wait."

\section{Auto $=1$}

Done $=0$

Level $=0$

$\mathrm{Ch} \$=\mathrm{m}^{\mathrm{N}} \mathrm{n}$ "

GOSUB Vmax_min_ptp

No_o_bins $=1024$

Min_bin = Pnts DIV 100

WHILE $\left(\mathrm{Ch} \$={ }^{\infty} n^{\infty}\right)$ OR $\left(\mathrm{Ch} \$={ }^{\circ} \mathrm{N}^{\infty}\right)$

WHILE NOT Done

Delta_ $v=V p t p /$ No_o_bins

Half $\bar{b}_{\text {in }}=$ No_o_bins DIV 2

MAT Hist_ary $=(0)$

FOR $I=1$ TO Pnts

Level $=1+\mid N T$ ( $($ Wave $\left.(1,2)-V m i n) / D_{\text {Delta_}} v\right)$

If Level >No_o_bins THEN

END IF Level $=$ No_o_bins

NEXT I

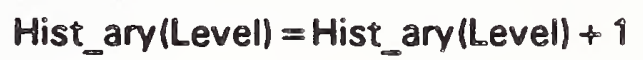

Bug $1=0$

IF Bug 1 THEN 
298 Hist message:

\section{NEXT I}

PRINTER IS PRT

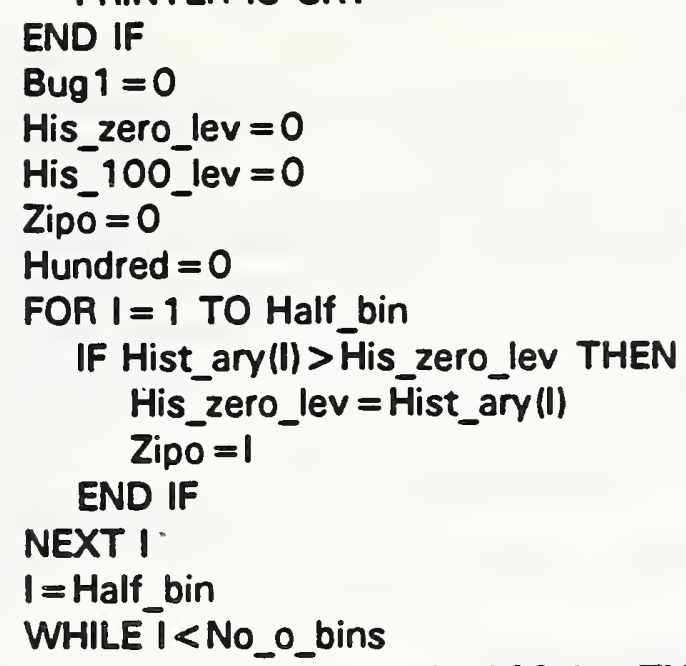


330 Hist_query: 1

332 !

\section{CLEAR SCREEN}

PRINT "The first waveform point $={ }^{"}{ }_{i} V_{-}$first; ${ }^{m}{ }^{*}{ }^{\circ} Y_{-}$units $\$_{i}{ }^{*}{ }^{*}$ PRINT

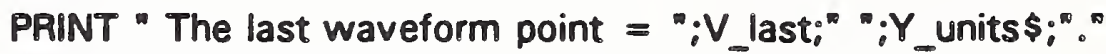

\section{PRINT}

344 PRINT

PRINT "or" :Delta_v_prc;" \% of the waveform peak-to-peak."

PRINT

PRINT "The 0\% level occurs at" "Volt_zi" ":Y_units\$;" with" ;His_zero_lev;"occurrences."

PRINT

PRINT "The $100 \%$ level occurs at" ;Volt_100;" "YY_units $\$ ; "$ with" ;His_100_lev;" occurrences."

INPUT "Is this an acceptable histogram $7^{*}, \mathrm{Ch} \$$

If $(\mathrm{C} h \$=" n$ ") OR (Ch\$ = "N") THEN

INPUT "How many histogram bins would you like to use?" ${ }^{\text {,No_o_bins }}$

Done $=0$

Auto $=0$

END IF

CLEAR SCREEN

RETURN

$386 \mathrm{Xy}$ _histogram: $\quad$ !

388 Range $=$ Wave(Pnts, 1$)$-Wave $(1,1)$

IF Range $>1 . E-50$ THEN

Base $=10^{\wedge}$ INT (LGT(Range))

SELECT Range

CASE $<=2$ "Base

Facto $=$ Base $/ 5$

CASE $<=5$ Base

Facto $=$ Base $/ 2$

CASE $<=10^{\circ}$ Base

Factor $=$ Base

END SELECT

ELSE

Factor $=1$

END IF

$X_{\min }=$ Factor $"($ INT (Wave $(1,1) /$ Factor $\left.)\right)$

$X_{\text {max }}=$ Factor "(INT(Wave(Pnts, , )/Factor))

Edge_It $=X \min -.55^{*}\left(X_{\max }-X_{\min }\right)$

Edge_ $\pi=X_{\min }-35^{*}\left(X_{\max }-X_{\min }\right)$

Delta_ $x=($ Edge_rt-Edge_It)/MAX(Hist_ary ("))

Coeff $=$ REAL (No_o_bins/(No_o_bins-1)/

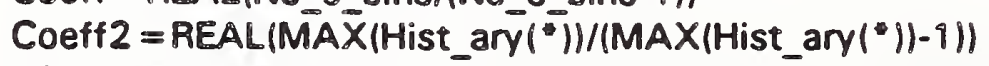

FOR $!=q$ TO No_o_bins

Histogram $(1,8)=$ Hist_ary $(1) \cdot$ Coeff $^{*}$ Delta_ $x+$ Edge_It

Histogram $(1,2)=(1-1)^{*}$ Coeff" Delta_ $v+V \min$ NEXT I 


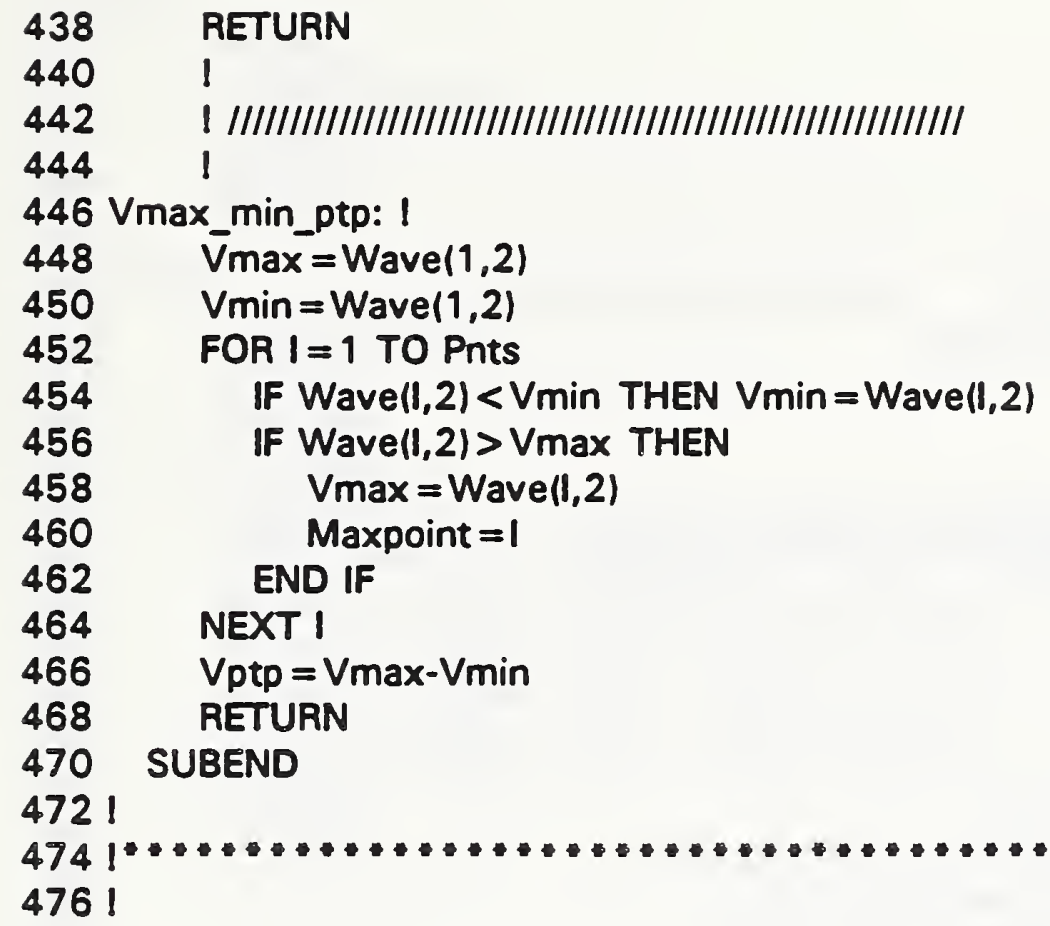




\section{B.7 MATH_OPS}

100

102

104

106

108

110

112

114

116

118

120

122

124

126

128

130

132

134

136

138

140

942

944

146 Date_line: !

148

150

152

154

156

158

160

162

164

166

168

170

172

174

176

178

180

182

184

186

188

190

192

194

196

198

200

202

204

206

208

1

I

END

1

I the data.

lo

I

DEG

!

Do_op:

1

LOOP
I RE-STORE "MATH_OPS:, 1400"

COM /Sys_msi/ Msi id \$[20]

COM /Sys/ Sys_id $\$ \overline{10}$ ]

COM Anterrupts/ INTEGER Intr_prty

OUTPUT KBD USING "K,\#";"SCRATCH KEYE"

CONTROL KBD, 15;0l sets the color of the soft keys

CONTROL KBD, $2 ; 1$

Intr_prty $=1$

CALL Do_op

OUTPUT KBD USING "K,\#";"LOAD KEYE"! restore the typing aid keys

PRINT TABXY(1,5);"END of program. So long."

MASS STORAGE IS " $: 1400^{\circ}$

1 This program performs simple math operations on waveform data.

I You may add, subtract, multiply, or divide (non-zero) the data

I by a constant of you may integrate, differentiate of time shift

I S. M. Chesnut

I May 21,1991

SUB DO_OP

OPTION BASE 1

COM /Flgs/ Stp_flg

COM /Interrupts/ INTEGER Intr_prty

COM /Files/ Diskdrive $\$[20]$, Filename $\$[14]$, Ms path $\$[500]$

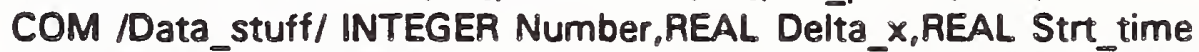

COM /Data_vars/ REAL Wave(4096,2),New_wave(4096,2),INTEGER Loaded

INTEGER Local_prty,Basketsize

DIM Data_id\$[40],Test\$[20],Ch\$[1]

REAL Last_pnt,Wave_int, Time_plc

OFF KEY

OFF KNOB

OFF KBD

Interrupted $=1$

Local_prty = Intr_prty

Basketsize $=4096$

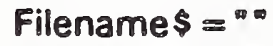

Diskdrive $\$=$ *

Loaded $=0$

Number $=0$

IF Interrupted THEN GOSUB Menu

ON KEY 9 LABEL "EXIT "LOCal_prty + 3 GOTO Ret 


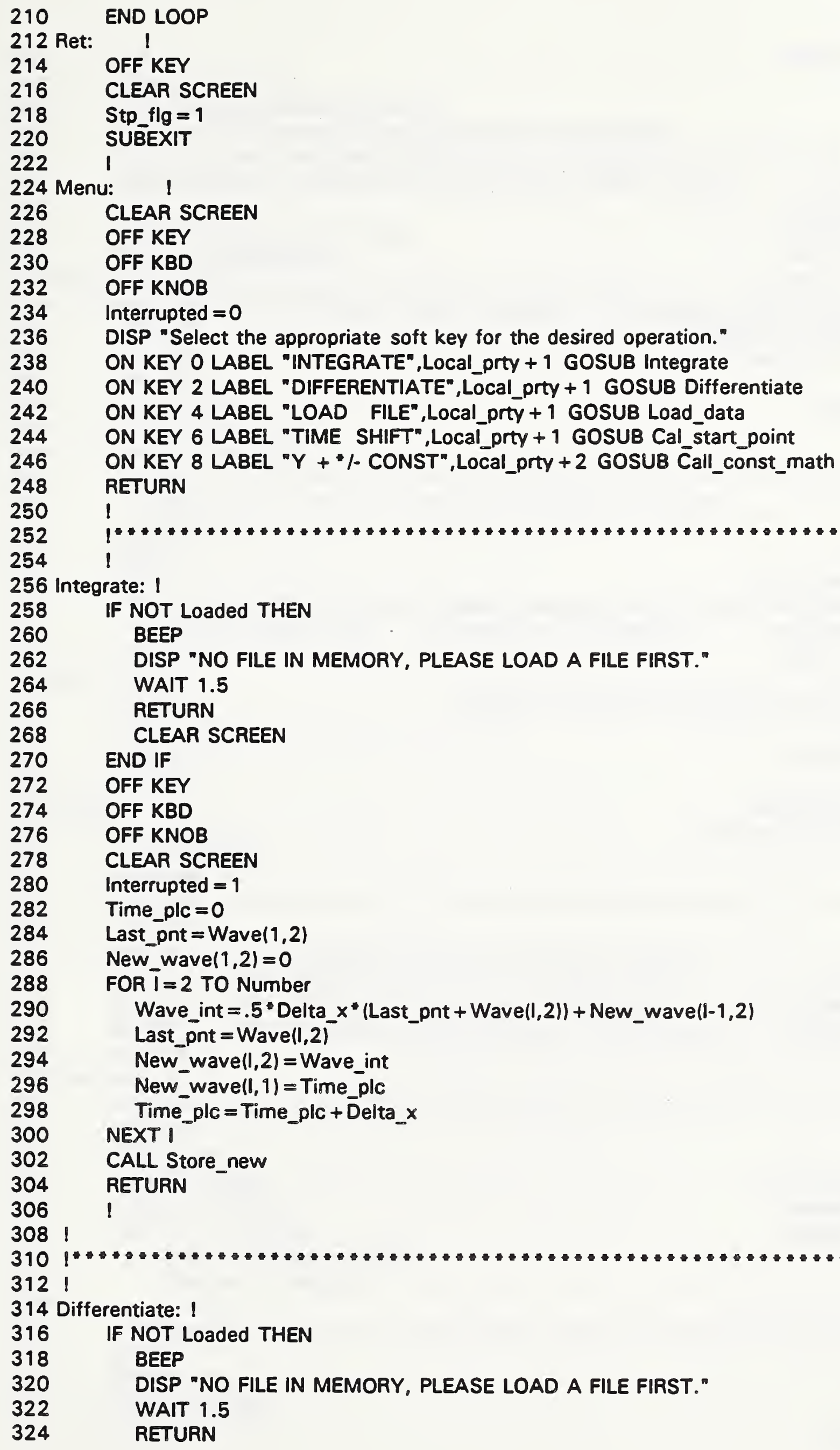


$400 \quad$ Intr_prty $=$ Local_prty +2

402 CALL Start point

404 Intr_prty $=$ Local_prty

406 RETURN

\section{END IF}

OFF KEY

OFF KBD

OFF KNOB

Interrupted $=1$

Time_plc $=$ Delta $\_x$

New_wave $(1,2)=0$

New_wave $(1,1)=0$

FOR $\bar{I}=2$ TO Number

New_wave $(1,2)=($ Wave $(1,2)-$ Wave $(1-1,2)) /$ Delta_ $x$

New_wave $\left(I_{1}, y\right)=$ Time_plc

Time_plc $=$ Time_plc + Delta_ $x$

NEXT I

CALL Store_new

RETURN 1

1

\section{Loaded $=1$}

lnte $p$ pty $=$ Local prty +2

CALL Load_disk_data(Wave("),Basketsize,Data_id\$,0)

intr_prty = Local prty

If Number $=0$ THEN

BEEP

DISP "NO FILE WAS READ, PLEASE tRY AGAIN."

WAIT 1.5

END IF

GOTO Load_data

REDIM Wave(Number, 28

REDIM New_wave(Number,2)

RETURN

1

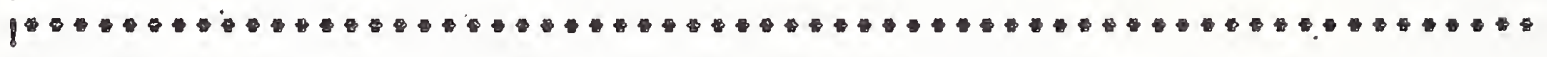
1

$408 \quad 1$

410

412

414 Call

416 IntP_prty = Local_prty +2

418 CALL Const_math

420 IntP_prty = Local_prty

422 RETURN

424 SUBEND

$426 \quad$

428

430

432

434

436

OPTION BASE 1 


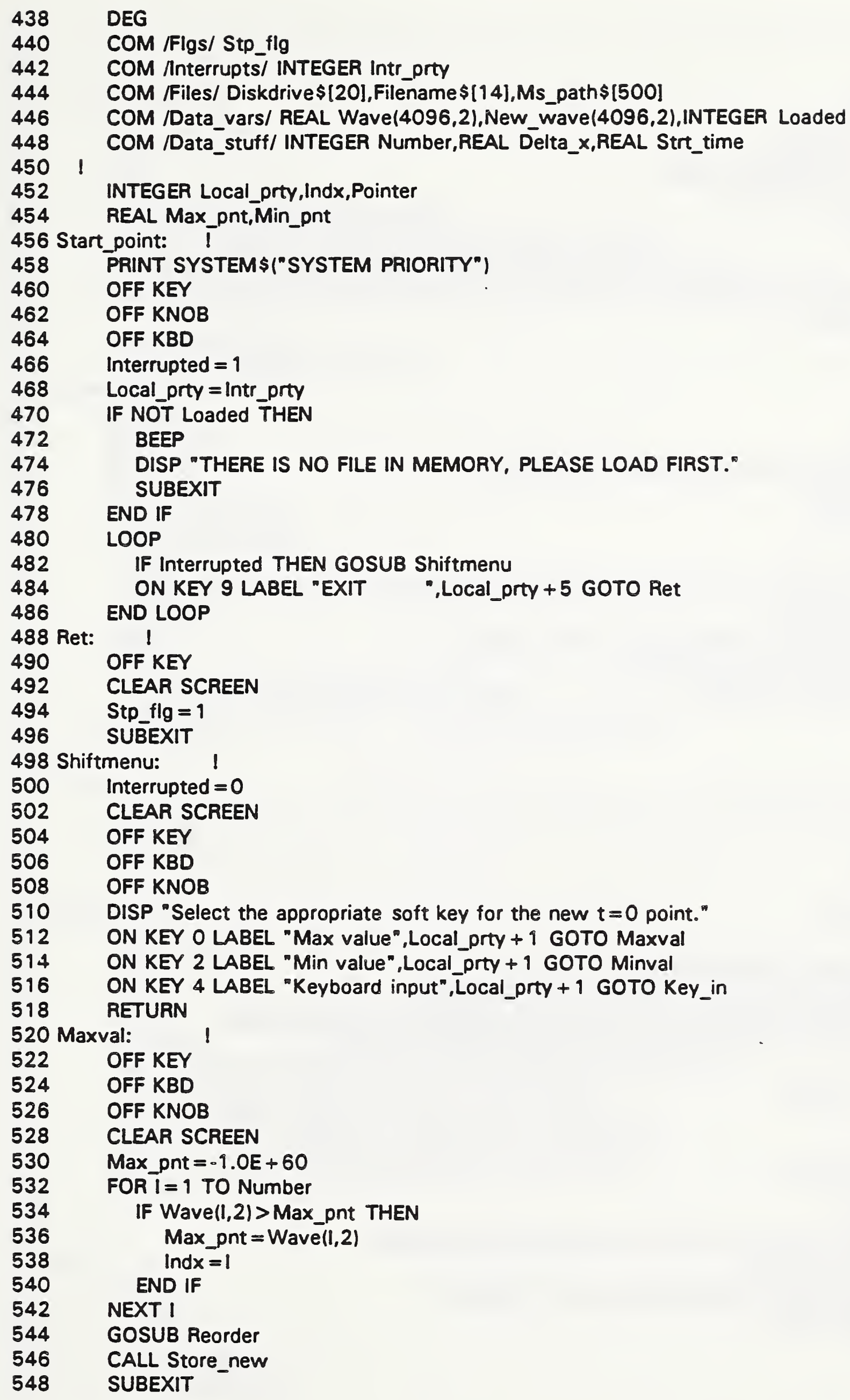




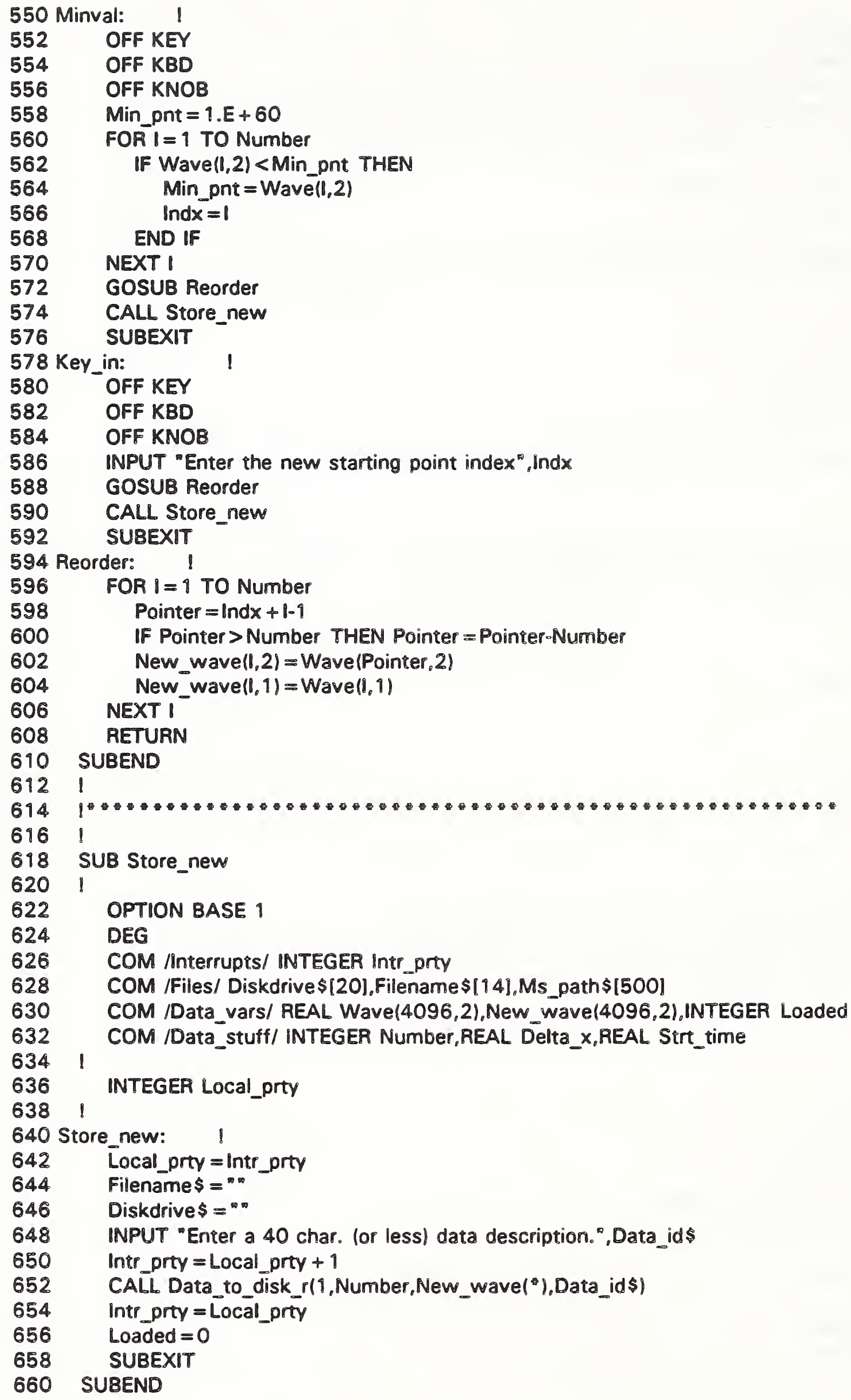




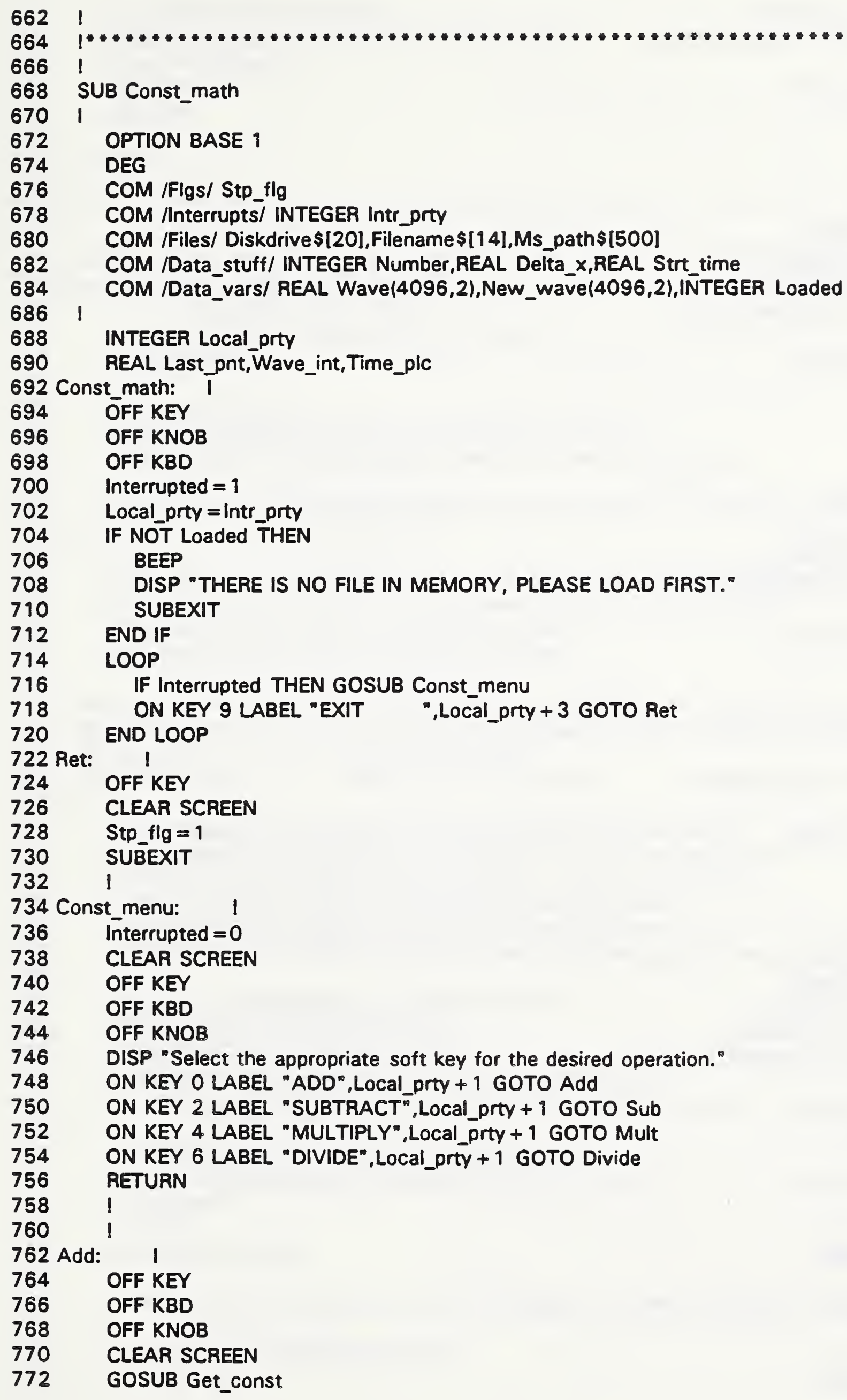




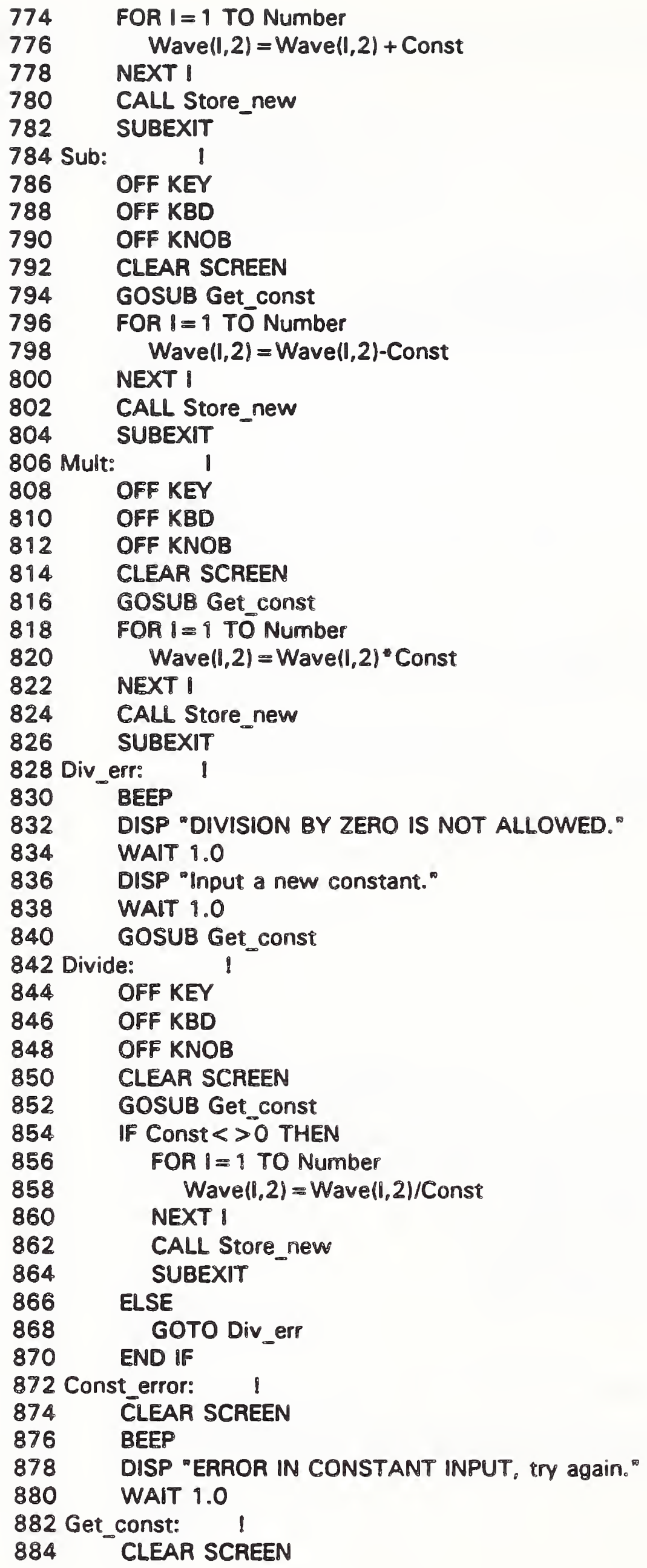




$$
1
$$

SUBEND

!

1

906 SUB Load_disk_data(Basket_file(*),INTEGER Basketsize,Data_id\$,INTEGER Flg)

908 Load_disk_data: 1 Original: 13 Nov 1984

910

912

914

916

918

920

922

924

926

928

930

932

934

936

938

940

942

944

946

948

950

952

954

956

958

960

962

964

966

968

970

972

974

976

978

980

982

984

986

988

990

992

994

996

I Revision: 02 Dec 1987

IThis routine will enter data files from the disk

OPTION BASE 1

I

COM /Sys/ Sys_id\$

COM /History/ Status\$[1],Time_orgn\$[8],Date_orgn\$[1 1]

COM /History/ Time_chng\$[8],Date_chng\$[11],Description\$[160]

1

COM Rabels/ Labels\$(30)[60],INTEGER Lbl_count,REAL Lbl_addr $(30,6)$

ILbl_addr: $x, y$, pen, size, LDIR, LORG

1

COM /Data_param/ INTEGER Datacount,Filesize,Curvecount,Roster(17,4)

COM /Data_param/ REAL Sym_size,Symbol\$(17)(2),Curve_id \$(17)(40]

COM /Data_param/ REAL Xmin_data,Xmax_data

COM /Data_param/ REAL Ymin_data,Ymax_data 1

IRoster: Curve\#, Start Addr in File( $\%$, Datacount, and PEN

ISymbol\$(i) $=$ " " or " $Y " \Rightarrow>$ no symbol, connect pts

ISymbols(i) $="{ }^{*} Y^{*}=>$ "symbol, connect pts

ISymbols(i) $="{ }^{\prime \prime} \mathrm{N}^{*}=>$ "symbol, do not connect pts

1

COM /Background/ Graphtype \$[12],Margins\$(2)[10],Papersize\$[1]

COM /Background/ REAL Pen_speed,INTEGER Backgnd_pen,Auto_time

COM /Background/ INTEGER Auto_file,REAL $X$ _cross_y,Y_cross_ $\bar{x}$

COM /Background/ Xgrid_tick\$[4],INTEGER Xmajor,Xminor

COM /Background/ Ygrid_tick\$[4],INTEGER Ymajor,Yminor

COM /Background/ REAL Xmin_graph,Xmax_graph,Ymin_graph,Ymax_graph

I

COM /Bugs/ INTEGER Bug1,Bug2,Bug3,Printer

COM /Interrupts/ INTEGER Intr_prty

COM /Enlarge_file/ INTEGER Overflow

COM /Files/ Diskdrive $\$[20$ ],Filename $\$[14$ ],Ms_path $\$$ [500]

COM /Data_stuff/ INTEGER Number,REAL Delta_x,REAL. Strt_time 1

INTEGER R,Hold_size,Local_prty,Allocated,FIs_cnt

DIM AC\$[5],Tempfile\$[10],Mask\$[10],Ftype \$[5],FIs\$(1)[14]

REAL Dtime

OFF KEY

Local_prty $=$ Intr_prty

I

ISelect the disk drive where the data exists

1

IF Overflow $<>0$ THEN Overflow $=0$

Hold_size $=0$ 


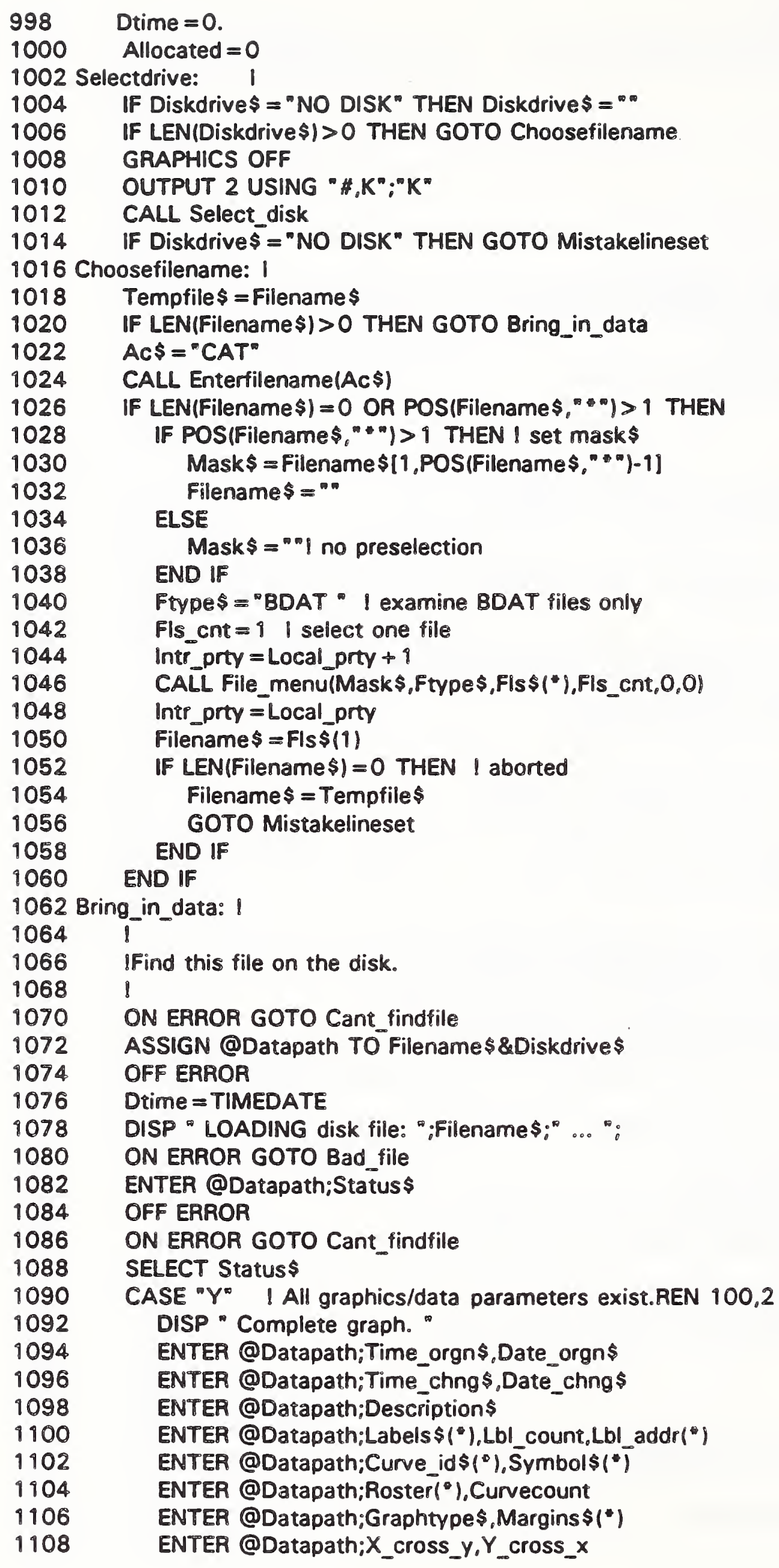



ENTER @Datapath;Xmin_graph,Xmax_graph ENTER @Datapath;Ymin_graph,Ymax_graph

1124 Bad_file: DISP CHR\$(12)

1126

1128

1130

1132

1134

1136

1138

1140

1942

1944

9146

1148

1150

1152

9154

1156

1158

1160

1162

1164

1166

1168

1170

1172

1174

1976

1178

1180

9182

1184

1186

1188

1190

1192

1194

1196

1198

1200

1202

1204

1206

1208

1210

1212

1214

1216

1218

1220
CASE " $N$ " I Only data parameters exist. DISP " RAW data."

CASE ELSE

DISP "Data file is not recognized, entry aborted.";

DISP " ...continue."

BEEP

PAUSE

OFF ERROR

GOTO Mistakelineset

END SELECT

1

ENTER @Datapath;Data_id\$

IF Flg THEN

ENTER @Datapath;Delta_x

ENTER @Datapath;Datacount

Hold_size $=$ Datacount

ELSE

ENTER @Datapath;Datacount

ENTER @Datapath;Hold_size

END IF

IF NOT Allocated THEN

IF Datacount $>=1$ AND Hold_size $>=1$ THEN

ALLOCATE Holding_file(Hold_size,2)

ELSE

ALLOCATE Holding_file $(1,2)$

END IF

Allocated $=1$

END IF

ENTER @Datapath;Holding_file(")

ASSIGN @Datapath TO.

OFF ERROR

IF NOT FIg THEN

Delta_ $x=$ Holding_file $(2,1)$-Holding_file $(1,1)$

Strt_time $=$ Holding_file $(1,1)$

END IF

IF Datacount $=0$ THEN Mistakeline

1

(Copy data from Holding_file $\left({ }^{\circ}\right)$ to Basket_file $(")$

I

MAT Basket_file $=(0$.

IF Datacount $>$ Basketsize THEN IReceiving file too small.

Allocated $=0$

DEALLOCATE Holding_file(")

DISP " DATA FILE overflow, new data discarded. ";

DISP " (continue) "

BEEP

PAUSE

IF Status $\$=$ " $Y$ " THEN

Curvecount $=0$

MAT Roster $=(0)$

END IF 


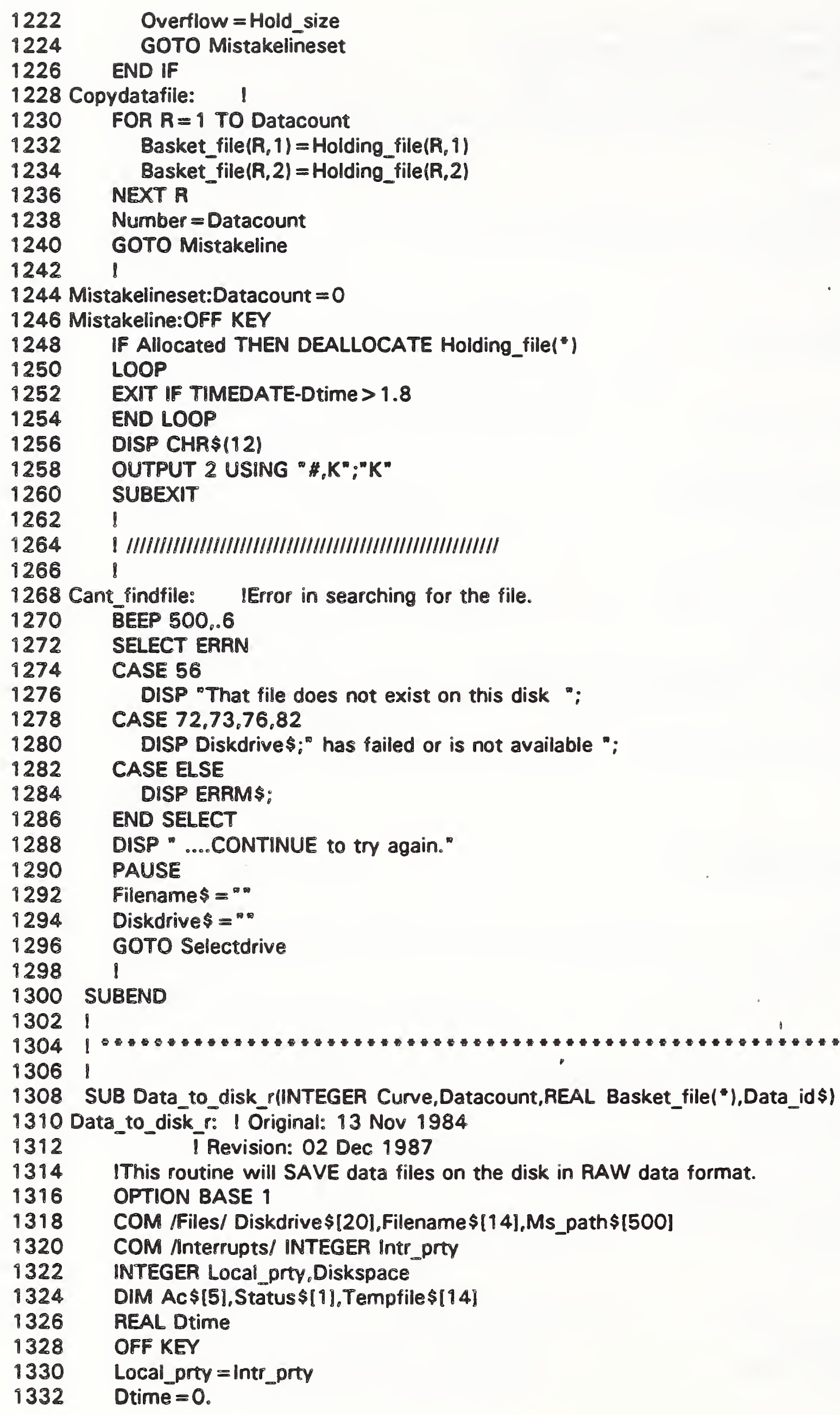


ISelect the disk drive for data storage

1340 Selectdrive: !

1342 GRAPHICS OFF

1344 OUTPUT 2 USING "\#,K";"K"

1346 CALL Select_disk

1348 IF Diskdrive $\$=$ "NO DISK" THEN GOTO Mistakeline

1350 Choosefilename: 1

1352 AC $\$=$ "ABORT"

1354 Tempfile $\$=$ Filename $\$$

1356 CALL Enterfilename(AC\$)

1358 IF LEN(Filename $\$$ ) $=0$. THEN

1360 Filename $\$=$ Tempfile $\$$

1362

1364

GOTO Mistakeline

1366 Send to disk: I Create file and save information.

1368

1370 ON ERROR GOTO Cant_savedata

1372

1374

1376

1378

1380

1382

1384

1386

1388

1390

1392

1394

1396

1398 Mistakeline:OFF KEY

1400 LOOP

1402 EXIT IF TIMEDATE-Dtime $>1.8$

1404 END LOOP

1406 DISP CHR\$(12)

1408 OUTPUT 2 USING "\#,K";"K"

1410 SUBEXIT

1412

1414

1416

1418 Cant_savedata: I

1420

1422

1424

1426

1428

1430

1432

1434

1436

1438

1440

1442

1444 1

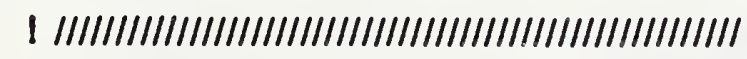

BEEP 500,.6

SELECT ERRN

CASE $72,73,76,78,81,82,90,93$

DISP Diskdrive ; $^{n}$ has failed or is not available "; DISP " ....CONTINUE to try again."

CALL Pause_key_on

Filename $\$=$ Tempfile $\$$

CASE 84,85

DISP " This disk is not initialized ";

DISP "...CONTINUE to try again."

CALL Pause_key_on

Filename $\$=\overline{T e m p}$ file $\$$

CASE 55,64 


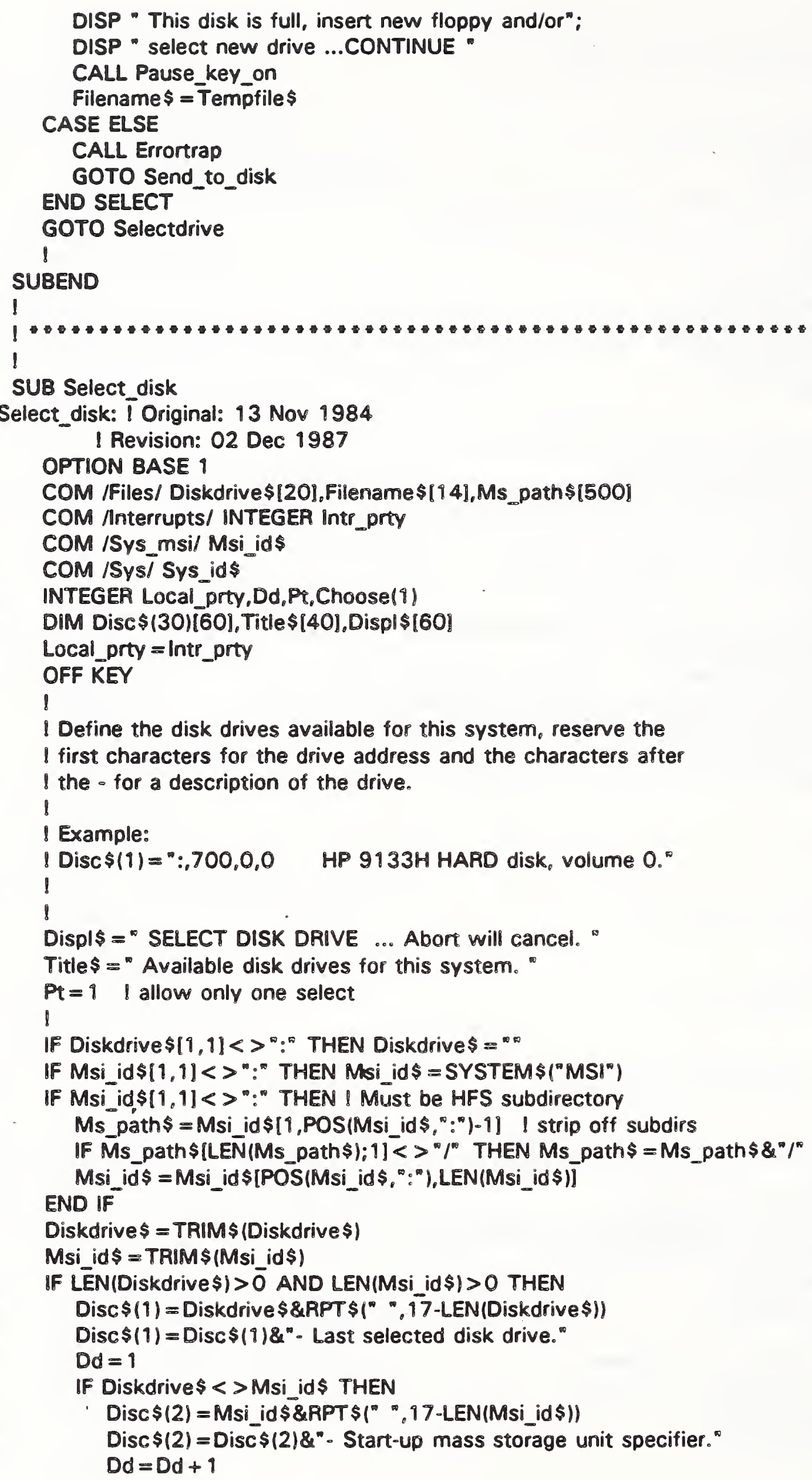


1650 Diskselected:OFF KEY

1652

1654

1656

1658

1660

1662 SUB Enterfilename(AC\$)

I Follow format with - after unit specifier, description is I optional but recommended.

$D d=D d+4 \quad I$ add the number of drive specifiers above !

IF Sys_id $\$ 1,4]<>$ "S300" THEN

Disc $\$(D d+1)=":, 4,1$

Disc $\$(D d+2)=":, 4,0$

- LEFT internal series 200"

$D d=D d+2$

\section{END IF}

1 .

CALL Menu_scroll(Displ\$,Title\$,Disc \$("),Dd,Pt,Choose ("))

IF $P t=0$ THEN

\section{ELSE}

Dd $=$ POS (Disc $\$$ (Choose (Pt)),"-") - 9 ! find -

IF Dd $>5$ THEN I valid msus

\section{ELSE}

$$
\text { Diskdrive } \$=\text { TRIM } \$ \text { (Disc } \$ \text { (Choose (Pt))[1,Dd]) }
$$

DISP " ERROR in reading MSUS from string, - chr not found. "

BEEP

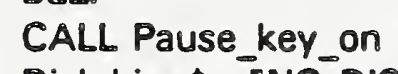


COM /Files/ Diskdrive $\$\left[20\right.$ ],Filename $\$[14], M s \_p a t h \$[500]$

COM /nterrupts/ INTEGER Intr_prty

INTEGER I,Ascii_num,Maskflag,Namelength

DIM Test\$[256],Hfs_temp\$[161]

Namelength $=10$

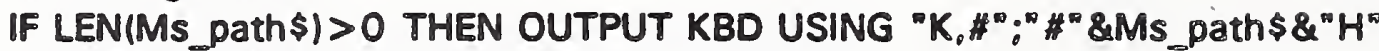
DISP " ENTER HFS directory PATH (no file)":

IF AC\$ $<>$ "PATH" THEN

DISP ". ENTER / for HFS ROOT or null for LIF...":

END IF

LINPUT His_temp\$

Hfs_temp $\$=$ TRIM $\$$ (Hfs_temp\$)

IF LEN(Hfs temp\$) $>0$ THEN

if LEN(Hifs_temp\$1>1 AND Hfs_temp\$[LEN(Hfs_temp $\left.\$)_{\theta} 1\right]<>"{ }^{\circ}$ THEN

His_temp $\$=H f s_{-}$temp $\$ \&^{n} /{ }^{\circ}$

END IF

IF LEN(His_temp $\$)=1$ THEN His_temp $\$=" *$

Namelength $=14$

END IF

IF AC\$ $=$ "PATH" THEN

Ms_path $\$=H f s_{-}$temp $\$$

SUBEXIT

END IF

IF LEN(Filename\$) $>0$ THEN OUTPUT KBD USING ${ }^{\infty} K_{0} \#^{\infty} ;{ }^{\infty}{ }^{\infty}$ \&Filename $\$ \&^{\infty} H^{\infty}$

SELECT AC\$

CASE "CAT"

DISP "(ENTER CAT mask OP ENTER null to CAT)":

CASE "ABORT"

DISP "(ENTER null to ABORT) ":

CASE "VALID"

DISP "(must be a VALID namel)":

END SELECT

UINPUT Test\$

Test $\$=$ TRIM $\$($ Test $\$)$

IF LEN(Test $\$$ ) $=0$ AND AC $\$=$ "VALID THEN GOTO Enterfilename

If LEN(Test $\$$ ) $=0$ THEN Abortline

If LEN(Test\$) > Namelength THEN

BEEP

DISP "ERROR in NAME ENTRY - max ";Namelength;" chars, you have "; DISP LEN(Test $\$ 1 ;$

WAIT 1.8

OUTPUT 2 USING "K, \#" " \#" \&Test\$\& $\& H^{\infty}$

GOTO Efn

END IF

IF POS(Test\$," ") $>1$ THEN

Test $\$=$ Test $\$\left[1\right.$, POS(Test\$。 ${ }^{\circ}$ " $)$ 1]

Maskflag $=1$

ELSE

Maskflag $=0$

END IF

FOR $I=1$ TO LEN(Test\$

Ascii_num = NUM(Test $\$$ (II)

SELECTT Ascii_num

CASE 65 TO $90,95,97$ TO 122,48 TO 57 
BEEP

DISP "ERROR in NAME ENTRY-ILLEGAL CHARACTERS, TRY AGAIN."

\section{WAIT 1.8}

OUTPUT 2 USING "K, \#";"\#"\&Test\$\&"H"

GOTO Efn

END SELECT

NEXT 1

IF Maskflag THEN

Filename $\$=$ Test $\$ \& n * n$

ELSE

Filename $\$=$ Test $\$$

END IF

Ms_path $\$=$ Hfs_temp\$

SUBEXIT

1814 Abortline:Filename $\$=$ "

1816 IF Ac $\$=$ "CAT" THEN Ms_path $\$=$ Hfs_temp $\$$

1818

1820

1822

1824

1826

1828

1830

1834

1836

1838

1840

1842

1844

1846

1848

1850

1852

1854

1856

1858

1860

1862

1864

1866

1868

1870

1872

1874

1876

1878

SUBEXIT

SUBEND

1

1

SUB File_menu(Mask\$,Ftype $\$, F I s \$(*)$,INTEGER Fls_cnt,Dir_on,Prt_on)

le_menu: 1

I Original: 29 Jun 1987, G. Koepke

I Revision: 02 Dec 1987, 07:00

OPTION BASE 1

DEG

COM /Sys/ Sys_id\$[10]

COM /Files/ Diskdrive \$[20], Filename $\$[14], M s$ _path $\$$ [500]

COM /nterrupts/ INTEGER Intr_prty

COM /Bugs/ INTEGER Bug1,Bug2,Bug3, Printer

DIM Directory \$(600)[80],Bd\$(600)(71]

DIM D\$[80],T\$[51],Ids\$[40],Stat\$[1],Test\$[256]

INTEGER Bd_cnt,File_cnt,I,C_cnt,CO(1),Format_error,End_search

IF FIs_cnt $>0^{-}$THEN ALLOCATE INTEGER Choose(FIs_cnt)

1

! Catalog the disk specified

1

End_search $=0$

REPEAT I Generate path to file and extract file name.

ON ERROR GOTO Cat_errors

DISP " Reading the Directory ..."

IF LEN(Ms_path $\$$ ) $>0$ THEN

MASS STORAGE IS Ms_path\$[1,LEN(Ms_path\$1-1]\&Diskdrive\$

$$
\text { ELSE }
$$

MASS STORAGE IS Diskdrive\$

END IF

CAT TO Directory \$("):NO HEADER,COUNT File_cnt OFF ERROR

1882

1884

9886

1888

1890

1892

1

I set up array of legal file names.

1

Bd $\mathrm{cnt}=0$

MÄT Bd\$ $=\left({ }^{\circ \nabla}\right)$ 


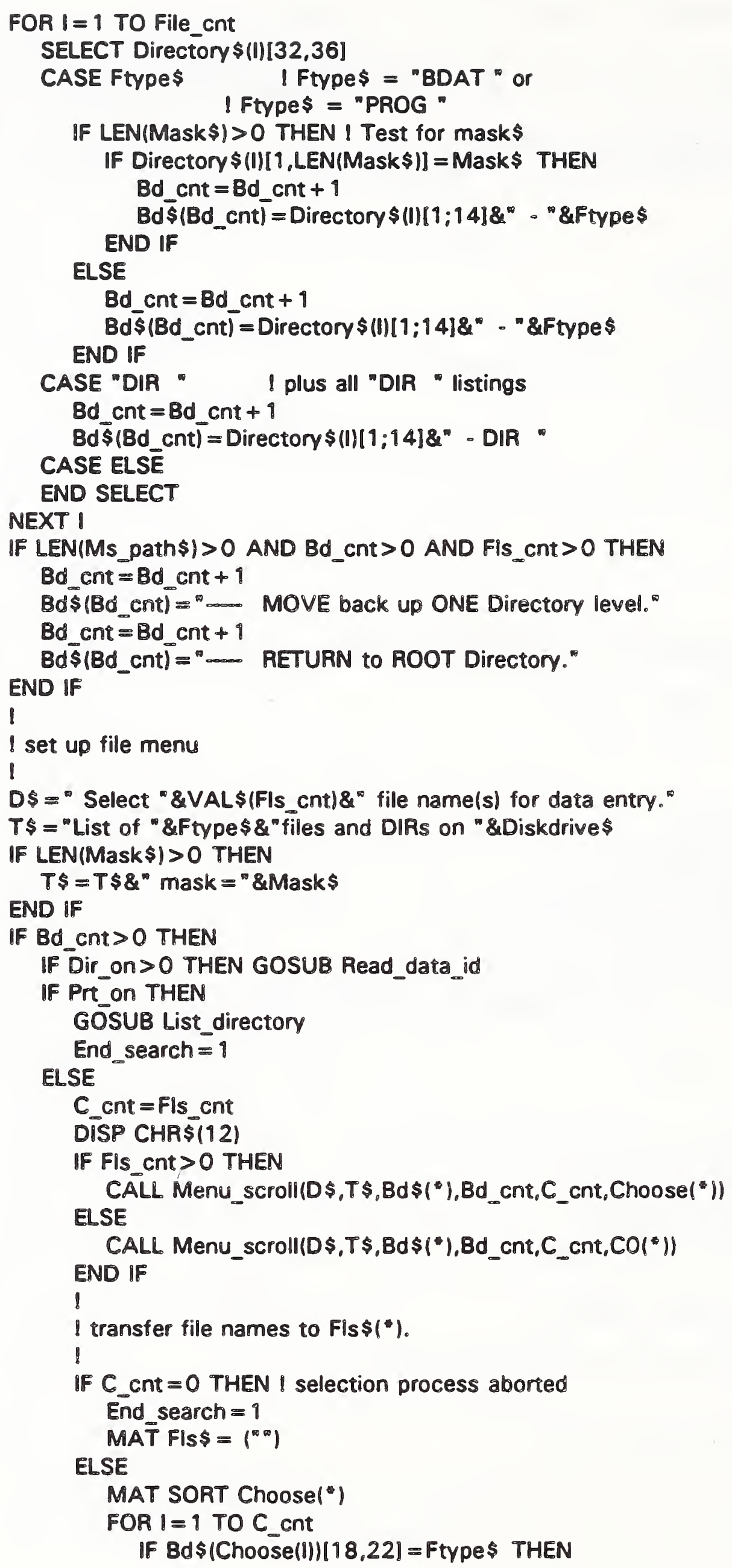


Fis $\$(I)=$ Bd $\$($ Choose $(1))[1 ; 14]$

End_search $=1$

2010

2012

ELSE $T$ it must be a Directory or message.

SELECT Bd\$(Choose(I)) $[18,22]$

CASE "up ON" I move up one directory

LOOP

2018

2020

2022

2024

2026

2028

2030

2032

2034

2036

2038

2040

2042

2044

2046

2048

2050

2052

2054

2056

2058

2060

2062

2064

2066

2068

2070

2072

2074

2076

2078

2080

2082

2084

2086

2088

2090

2092

2094 Read_data_id: 1 This routine expects to see Ids\$ from

2096

2098

2100

2102

2104

2106

2108

2110

2112

1 GRAPH_DATA raw data files.

DISP CHR\$(12)

UNTIL End_search

SUBEXIT

END IF

END IF

ELSE

DISP " This directory contains no ";Ftype $\$ ;$ " files ... "

WAIT 2.5

End_search $=1$

Ms_path $\$=$ Ms_path $\$ 1$
EXIT IF LEN(Ms_path $\$ 1=0$

Test $\$=$ Ms_path $\$$ [LEN(Ms_path $\$$ ); 1 ]

EXIT IF Test $\$=" / n$

END LOOP

CASE "ROOT " I jump to root directory

Ms_path\$ = " "

CASE "DIR " I add directory to Ms_path\$

Test $\$=$ TRIM $\$$ (Bd \$ (Choose $(I))[1,14])$

Ms_path $\$=$ Ms_path $\$ \&$ Test $\$ \&^{*} /{ }^{\circ}$

CASE ELSE

DISP "ERROR in directory jump"

PAUSE

END SELECT

I = C_cnt

END IF

Cat_errors:I

DISP " ERROR ... ":ERRM\$
BEEP

CALL Pause_key_on

DISP CHR\$(1)

C_cnt $=0$

MAT FIs $\$=(" \sqcap)$

SUBEXIT

!

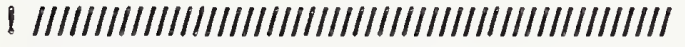
1

DISP "Reading file contents ... Please stand by. "

PRINT TABXY $(1,18) ; "$ Reading \#";

FOR $\mid=1$ TO Bd_cnt $\mid$ each BDAT file

PRINT TABXY $(11,18)$;

PRINT USING "3D,4A,3D,2A, \#" $11, "$ of "Bd_ent," " "

Ids $\$=$ "Data not recognized."

IF Bd\$(I) $[18,22]=$ "BDAT " THEN

ON ERROR GOTO Not_recognized

ASSIGN @lo_path TO Bd\$(1) $[1 ; 14]$

ENTER @lo_path;Stat\$ 
2130 Not_recognized:ASSIGN @lo_path TO.

2132

2144 Other_format:!

2946

2948

2150 Go on:NEXT I

2152 PRINT TABXY(1,18);RPT \$(" "40):

2154 DISP CHR\$(12):

2156 RETURN

2158

2160

2162

2964 Interpret_ $1: 1$ This is used to interpret ID strings.

2166 Format_error $=1$

2968 I identify this particular format

2970 RETURN

2972 \&

2974 I IIIIIIIIIIIIIIIIIIIIIIIIIIIIIIIIIIIIIIIIIIIIIIII

2976 !

2178 List_directory: 1 This routine will provide a tabular listing of

2180

2182

2184

2186

2188

2190

2992

2194

2196

2198

2200

2202

2204

2206

2208

DISP " Listing directory ..."

ON TIMEOUT 7.10 GOTO Printer_kaput

PRINTER IS Printer

PRINT USING $" /{ }^{\circ}$

PRINT TS

IF LEN(Ms_path\$) >0 THEN PRINT "HFS Path: "Ms_path\$

PRINT RPT $\$\left({ }^{\circ}={ }^{\circ}{ }_{8} 80\right)$

PRINT "File name";

IF Dir on THEN

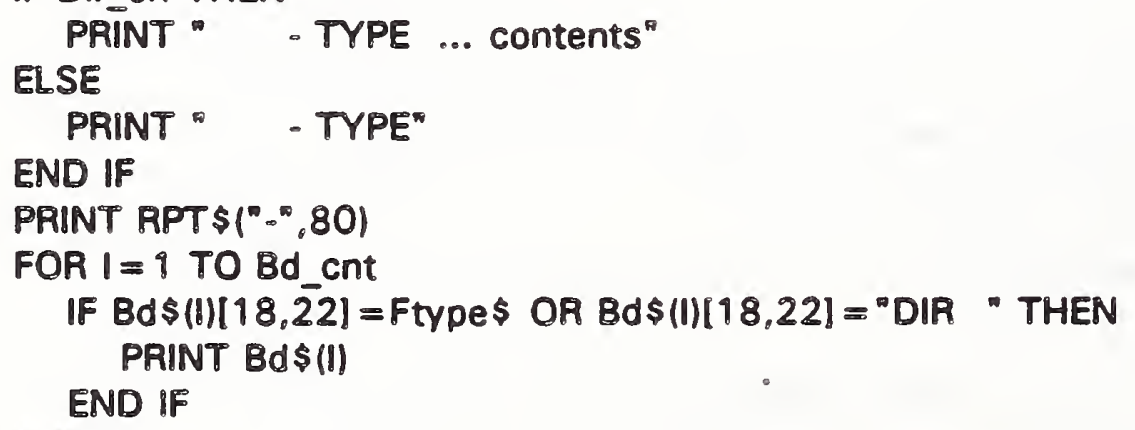

\section{NEXT 1}

PRINT RPT $\left.\$ 1^{\circ}{ }^{\circ}, 80\right)$

PRINT

PRINTER IS CRT

OFF TIMEOUT 7 
2232 Printer_kaput:DiSP " Printer not responding ... listing aborted. "

2234 BEEP

2236 WAIT 1.8

2238 OFF TIMEOUT 7

2240 RETURN

2242 SUBEND

2244 !

2246

2248 !

2250 SUB Menu_scroll(D\$,T\$,Items $\$(*)$, INTEGER Item_cnt,To_select,Choose (*))

2252 Menu_scroll: 1 Original: 22 Jun 1987, Galen Koepke, NBS $\overline{7} 23.04$

I A general purpose menu utility for scrolling items and

I selecting either a fixed number or a random number.

2264

1 of items.

I for fixed: To_select $>0$

1 for random: To_select $=-1$

2266

1 The items are arranged in screens of 15 items each and

$I$ the user may access screens via softkeys. There may be

2272

2274

2276

2278

I up to 40 screens or 600 items to choose from.

! Maximum sizes: $D \$[80], T \$[51]$, Items ( ${ }^{*}$ |[70]

I Items $\$\left({ }^{\circ}\right)$ contains the item descriptions

1 Item_cnt is the number of items in Items $\$(")$

I Choose (") is dimensioned to the number of required choices

$1 \quad$ and will be filled with the item numbers chosen.

I To_select is the number of required choices.

$$
1
$$

OPTION BASE 1

PRINTER IS CRT

DEG

GOSUB Def_variables

GOSUB Define_screens

GOSUB Make_selections

IF Null_file THEN I reset to zero

Item_cnt $=0$

Items $s(1)=$ " *

END IF

To_select $=0 \quad$ I no valid selections

\section{SUBEXIT}

1

\section{! IIIIIIIIIIIIIIIIIIIIIIIIIIIIIIIIIIIIIIIIIIIIIIII}

$$
1
$$

2320

2322

2324

2326 
Local_prty $=$ Intr_prty

If Local_prty $<1$ THEN Local_prty $=10$

IF LEN(SYS_id \$) $=0$ THEN SYS_id $\$=$ SYSTEM ("SYSTEM ID")

If Item_cnt $<1$ THEN

Null_file $=1$

Item_cnt $=1$

To_select $=0$

2356

2358

2360

2362

2364

ELSE

Items $\$(1)=\cdots$ Empty * *

Null file $=0$

END IF

2366

2368

2370

2372

2374

2376

2378

2380

2382

2384

2386

2388

2390

2392

2394 Define screens:l Set up screens of 15 items each.

2396

2398

2400

2402

2404

2406

2408

2410

2412

2414

2496

2418

2420

2422

2424

2426

2428

2430

2432

2434

2436

2438 Make selections:! MENU setup and use. 
2454 Key_loop: !

2456 ON KBD,Local_prty GOSUB Process_kbd

ON KNOB .01,Local_prty GOSUB Move_pointer

IF Random_select THEN

I set keys for random selection

DISP D\$

ON KEY KO LABEL "Select",Local_prty GOSUB Select_random

ON KEY KO + 9 LABEL "Accept",Local_prty GOTO Exit_line

ELSE I set key $K O$ for fixed selection

IF Skips < To_select THEN

DISP D\$

IF To_select $>1$ THEN

Test $\$$ = " Select "\&VAL\$(Skips + 1)\&" of "\&VAL\$(To_select)

ELSE

Test $\$="$ Select"

END IF

ELSE

ON KEY KO LABEL Test\$,Local_prty GOSUB Select_fixed

IF To_select $>0$ THEN

DISP " Selection process complete ..."

ELSE

DISP " Menu for information only ..."

END IF

ON KEY KO LABEL "Accept",Local_prty GOTO Exit_line END IF

END IF

2506

2508

2510

2512

2514

2516

2518

2520

2522

2524

2526

2528

2530

2532

2534

2536

2538

2540

2542

2544

2546

2548

2550

2552

2554

2556

2558

2560

IF Active_screen $<$ Screen_cnt THEN

ON KEYY KO + 1 LABEL" "Next Screen",Local_prty GOSUB Next_screen

ELSE

OFF KEY KO +1

END IF

IF Active_screen $>1$ THEN

ON KEYY KO + 2 LABEL "Last Screen",Local_prty GOSUB Last_screen

ELSE

OFF KEY KO +2

END IF

IF Skips $>0$ OR Random_select THEN

ON KEY KO + 3 LABEL " Reset Select",Local_prty GOSUB Select_reset ELSE

OFF KEY KO +3

END IF

IF To_select $>0$ OR Random_select THEN

ELSE

ON KEY KO + 4 LABEL." Abort" "Local_prty GOTO Escape_line

OFF KEY $\mathrm{KO}+4$

END IF

IF Screen_cnt $>2$ THEN

ELSE

ON KEYY KO + 6 LABEL "Jump to Screen",Local_prty GOSUB Jump_to_scn

OFF KEY KO +6

END IF

IF Exit_flag THEN Exit_line

GOTO Key_loop

(M)_line:Skips $=0$

MAT Choose $=(0)$

2564 To_select $=0$ 


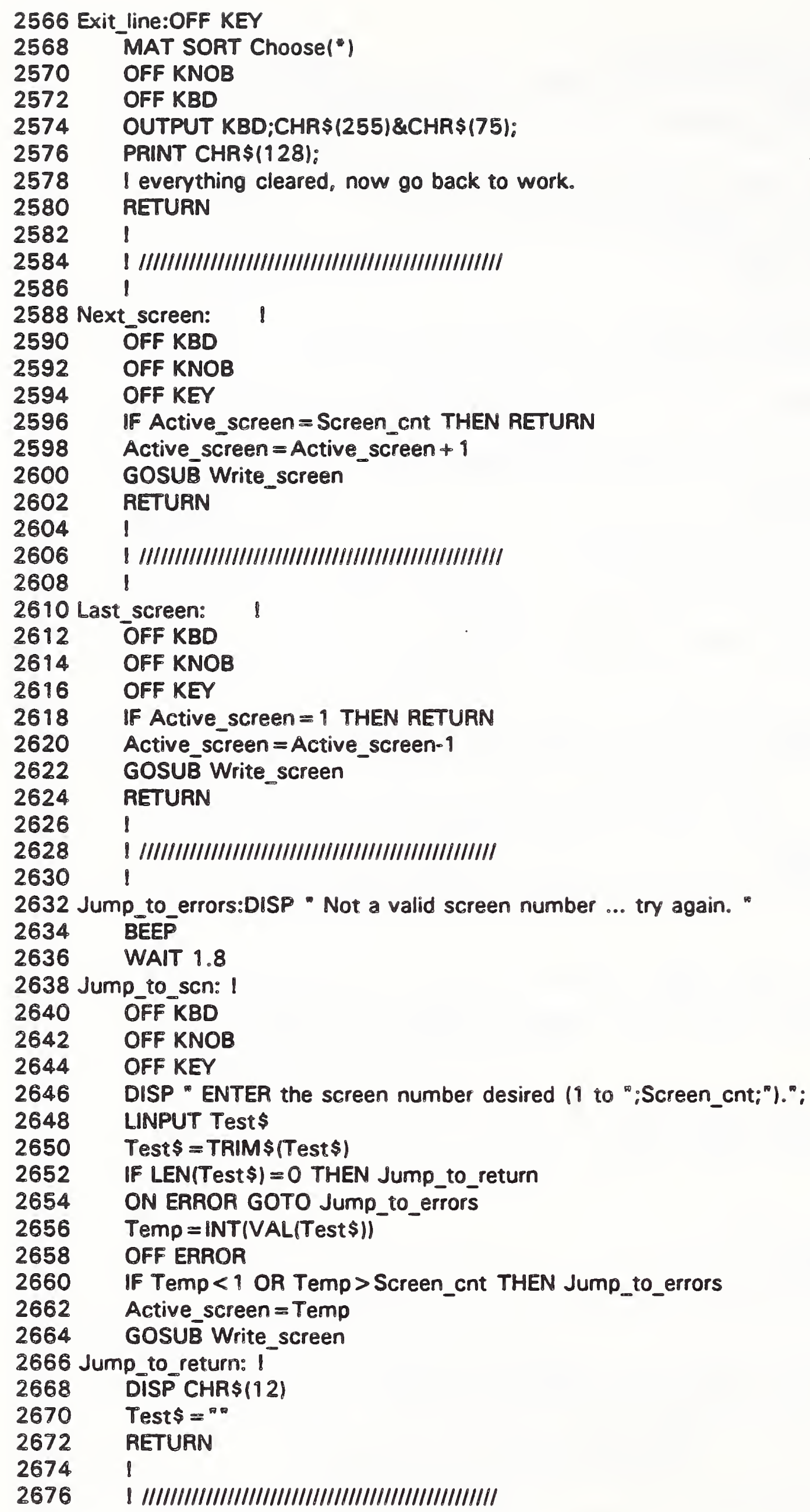


2680 Select_fixed:!

2682 OFFF KBD

2684 OFF KNOB

2686 OFF KEY

2688

2690

2692

2694

2696

2698

2700

2702

2704

2706

2708

2710

2712

2714

2716

2718

2720

2722

2724

2726

2728

2730

2732

2734

2736

2738

2740

2742

2744

2746

2748

2750

2752

DISP "NO additional selections for this screen."

BEEP

WAIT 2

DISP CHR\$(12); RETURN

END IF

IF Skips $=$ To_select THEN

IF To select $=0$ THEN

DISP "This menu is for information only,"; DISP " no selection allowed."

ELSE DISP "All selections have been filled,"; DISP " 'Select Reset' to repeat."

END IF

BEEP

WAIT 2

DISP CHR\$(12);

RETURN

END IF

Skips $=$ Skips +1

Choose (Skips) = First_item (Active_screen) + Pointer-First_line

PRINT CHR\$(129); I inverse video

PRINT TABXY(10,Pointer);Items $\$$ (Choose(Skips))

PRINT CHR\$(128);

PRINT TABXY(1,Pointer);

SELECT Pointer

CASE First_line

GOSUB Point_forward

CASE Last_line

GOSUB Point_backward

CASE ELSE

I move forward unless it requires wrapping to beginning.

If Skips-1 >0 THEN I check for selected items.

$\mathrm{I}=$ Pointer-First_line

LOOP

$K=0$

FOR $J=1$ TO Skips

IF First_item(Active_screen) $+1=$ Choose(J) THEN $K=1$ NEXT J

EXIT IF $K=0$

$I=1+1$

IF I + First_line > Last_line THEN $K=-1$

EXIT IF $K=-\overline{1}$

END LOOP

IF $K=0$ THEN

GOSUB Point_forward

ELSE

GOSUB Point_backward

ELSE

ND IF 
2804 Select_random:! 
OFF KNOB

OFF KEY IF Random_select THEN To_select $=0$

Skips $=0$

MAT Choose $=$ (999)

GOSUB Write_screen

RETURN

!

2920

2922 Process_kbd:I Allow use of arrows and enter key in addition to soft.

2924

2926

2928

2930

2932

2934

2936

2938

2940

2942

2944

2946

2948

2950

2952

2954

2956

2958

2960

2962

2964

2966

2968

2970

2972

2974

2976

2978

2980

2982

2984

2986

2988

2990 Point_forward:Knobcount $=5$

2992 ḠOSUB Move_pointer

2994 RETURN

2996 Point_backward:Knobcount $=-5$

2998 ḠOSUB Move_pointer

3000. RETURN

$3002 \quad 1$

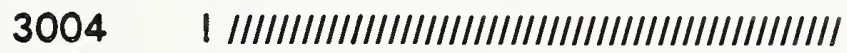

$3006 \quad 1$

3008 Jog_pointer:I Move the selection pointer on the active screen.

3010

3012

I without regard to selected values

IF Knobcount $>0$ THEN I Move forward 
3104 i home and clear screen

3902 Write screen:! Write the screen pointed to by Active_screen

I Move backward

$$
\text { ELSE }
$$$$
\text { Pointer }=\text { Pointer }-9
$$

\section{END IF}

IF Pointer $<$ First line THEN Pointer $=$ Last line

IF Pointer $>$ Last_line THEN Pointer $=$ First_line RETURN

$$
\text { I }
$$

[ IIIIIIIIIIIIIIIIIIIIIIIIIIIIIIIIIIIIIIIIIIIIIIIIIIIIII]

IF NOT Pointeractive THEN RETURN I No selections to be made. Knobcount $=$ Knobcount + KNOBX-KNOBY

IF ABS(Knobcount) $<4$ THEN RETURN

Last_pt $=$ Pointer

GOSUB Jog_pointer

IF Skips $>0$ THEN

LOOP

$$
J=\text { Pointer-First line }
$$

FOR $I=1$ TO Skips

If First item(Active screen) $+J=$ Choose(l) THEN $J=999$

\section{NEXT I}

IF $J=999$ AND Pointer $=$ Last pt THEN Pointeractive $=0$

EXIT If Pointeractive $=0$

If $J=999$ THEN GOSUB $\operatorname{Jog}_{-} p o i n t e r$

EXIT IF $J<>999$

END IF

END LOOP

Knobcount $=0$

OUTPUT KBD;CHR\$(255)\&CHR\$(84): I Bring screen home

IF Last_pt = Last_line THEN PRINT CHR\$(132):

PRINT " $:$

If Pointeractive THEN \& Pointer active

If Pointer = Last line THEN

ELSE

PRINT CHR\$ (1932):

PRINT CHR\$ (1928):

END IF

END IF

PRINT TABXY(1, Pointer):Marker\$:CHR\$ (928):

RETURN

I

I IIIIIIIIIIIIIIIIIIIIIIIIIIIIIIIIIIIIIIIIIIIIIIII

OUTPUT KBD;CHR\$(255)\&CHR\$(84)\&CHR\$ (255)\&CHR\$(75);

Knobcount $=$ KNOBX-KNOBY \& Clear knob and keyboard

Knobcount $=0$

Test $\$=$ KBD $\$$

Test $\$={ }^{\mathrm{m}}$

1

PRINT TABXY(1,First_line-1);CHR\$(132): item \#) Screen \#":

PRINT USING "\#,2D, $\overrightarrow{4 A}, 2 \mathrm{D}, 3 \mathrm{~A}^{*}$;Active_screen," of ":Screen_cnt;" $\left.\right|^{\text {" }}$ PRINT T\$:RPT \$ $1^{\circ}{ }^{\circ}, 51$-LEN(T\$));

PRINT TABXY(80, First_line-1);" 1 ":CHR\$(128): 
IF J = Last_item(Active_screen)-First_item(Active_screen) THEN PRINT CेHR\$(132);

PRINT TABXY(1, First_line + J);RPT $\$(" n, 80)$

3136

ELSE PRINT CHR\$(128);

END IF

PRINT TABXY(5, First_line + J);

PRINT USING "3D,A, \#";First_item(Active_screen) + J," I"

IF Random_select THEN FOR I $=\overline{1}$ TO TO_select IF First_item(Áctive_screen) $+\mathrm{J}=$ Choose(l) THEN END IF PRINT CHR\$(129);

$$
\text { NEXT I }
$$

END IF

PRINT TABXY(10,First_line + J); Items $\$$ (First_item(Active_screen) $+J$ )

PRINT TABXY(80,First_line + J);" $\mid "$;

$$
\mathrm{J}=\mathrm{J}+1
$$

UNTIL $J>=$ (Last_item(Active_screen)-First_item(Active_screen) +1$)$

Last_line $=$ Last_item (Active_screen)-First_item (Active_screen)

Last_line $=$ Last_line + First_line

!

I set marker to first non-selected item.

!

Pointeractive $=0$

IF To_select $>0$ OR Random_select THEN Pointeractive $=1$

IF Skips $>0$ AND Pointeractive $=1$ THEN $!$ find first non-selected item $J=0$

LOOP

Pointer $=$ First $\_$line $+J$

FOR I $=9$ TO Skips

IF First item(Active screen) $+J=$ Choose(l) THEN Pointer $=0$ NEXT I

EXIT IF Pointer $<>0$

$J=J+1$

IF First_line $+J>$ Last_line THEN

Pointeractive $=0$

Pointer $=$ First_line

END IF

EXIT If Pointer $<>0$

ELSE END LOOP

Pointer $=$ First_line

END IF

IF Pointeractive THEN

IF Pointer $=$ Last_line THEN 
3262 PUB Pause_kev_on

3264

3266

3268

3270

3272

3274

3276

3278

3280

3282

3284

3286

3288

3290

3292

3294

3296

3298

3300

3302

3304

3306

3310

3392

3314

3316

3318

3320

3322

3324

3326

3328

3330

3332

3334

3336

3338

3340

3342

3344

3346

3348

END IF

PAUSE

END IF

SUBEXIT

SUBEND

I

1

SUB Errortrap

CASE 54
ELSE

PRINT CHR\$(128);

END IF

PRINT TABXY(1,Pointer);Marker\$;CHR\$(128):

END IF

RETURN

SUBEND

1

1

SUB Pause_key_on

use_key_on: I Make sure that CONTINUE key exists.

I Original: 02 Dec 1987

OPTION BASE 1

COM /Sys/ Sys_id\$[10]

IF Sys id $\$[1,4]=$ "S300" THEN I reset to $\$ 300$ system keys

CONTROL KBD, 15:0

CONTROL CRT, $12 ; 2$

LOAD KEY

IF Sys id $\$[1,4]=" S 300$ " THEN I set to $\$ 200$ compatible keys

OUTPUT KBD USING "K, \#";"SCRATCH KEYX"

CONTROL KBD, $15 ; 9$

CONTROL CRT, 12;0

I Revisinal: 13 Nov 1984

I Revision: 02 Dec 1987

! Trap most errors here

OPTION BASE 1

COM /Files/ Diskơrive \$[20],Filename\$[14],Ms_path\$[500]

DIM File $\$[20]$, Test $\$[256]$, What $\$[20], A c \$[5]$

BEEP 400,06

SELECT ERRN

DISP "DUPLICATE FILE NAME: ";Filename\$;

DISP *...PURGE old one? (Y/N)";

LINPUT What

What $\$=$ TRIM $\$$ (What $\$$ )

SELECT What $\$ 1,1]$

CASE "Y" " $Y$ "

PURGE Ms_path\$\&Filename\$\&Diskdrive $\$$

CASE ELSE

$A C \$=$ "VALID"

CALL Enterfilename(AG\$)

END SELECT

CASE 52,53

DISP "Improper FILE NAME $\infty$ ENTER NEW FILE NAME"; 
3416 SUB Auto_format(Value)

3418 Auto_format: ! Original: 13 Nov 1984

3420

OUTPUT 2 USING "\#,K,K";"\#";Filename\$

LINPUT Filename\$

Filename $\$=$ TRIM $\$$ (Filename $\$$ )

\section{CASE 56}

DISP "FILE: ";Filename\$;" is not on this disk, please insert";

DISP " correct disk"

CALL Pause_key_on

\section{CASE 64}

DISP "This disk is full, PLEASE insert clean disk"

CALL Pause_key_on

CASE 56

DISP "DATA INPUT disk must be in drivell ";

DISP "...CONTINUE when ready."

CALL Pause_key_on

CASE $72,73,7 \overline{6}$

DISP Diskdrives:

DISP " is not available, type correct";

DISP " unit specifier (ie. ":,707,0').";

OUTPUT 2 USING "K,\#";Diskdrive $\$$

LINPUT Diskdrive\$

\section{CASE 80}

DISP "CHECK DISK drive doorl"

CALL Pause_key_on

CASE ELSE

DISP ERRM\$;" 'CONTINUE' when fixed"

CALL Pause_key_on

END SELECT

DISP CHRS(12)

SUBEXIT

SUBEND

1

$1 \cdot$

1

I Select the proper number of digits to display.

I This routine is used by several program sections to

I print numbers to the display.

1

SELECT ABS(Value)

CASE $>=1.0 E+5$

SELECT ABS(Value)

CASE $<9.0 E+90$

PRINT USING "\#,MD.4DESZ,2X";Value

CASE $<1.0 E+100$

PRINT USING "\#,MD.4DESZZ, $X "$;Value

CASE ELSE

PRINT USING "\#,MD.4DESZZZ";Value

END SELECT

CASE $>=1.0$

If Value $=$ PROUND $($ Value, -4$)$ THEN

IF INT(Value) $=$ Value THEN PRINT USING "\#,M5D,6X";Value

ELSE

PRINT USING "\#,M5D.4D, $X^{n}$;Value 


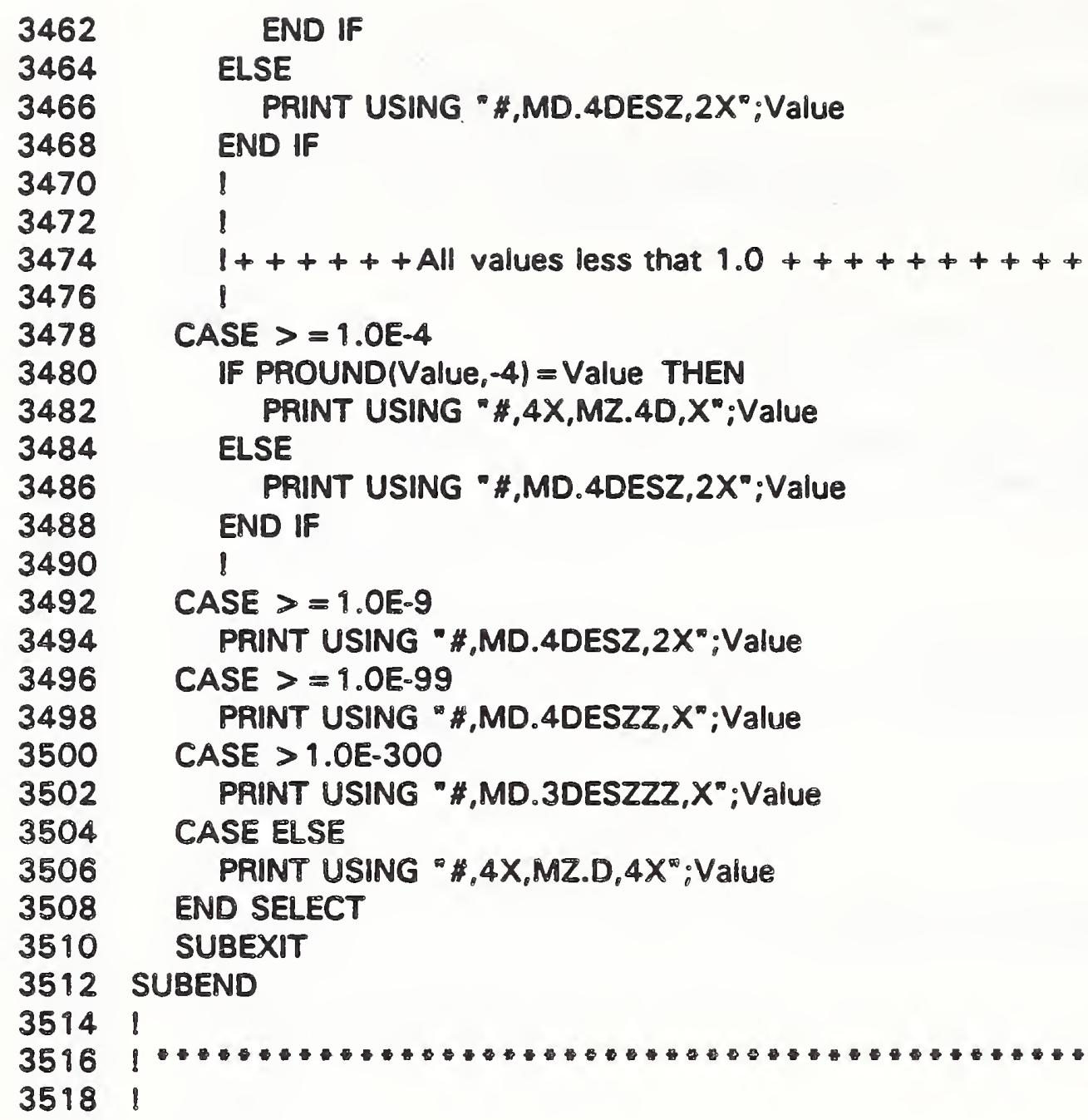


ISymbol\$(i) $={ }^{" m}$ or " ${ }^{\prime \prime}=>$ no symbol, connect pts

ISymbol $\$(i)="{ }^{*} \gamma^{*}=>$ "symbol, connect pts

214 ISymbol $\$(i)={ }^{*} N^{*}=>$ symbol, do not connect pts

216

COM /Background/ Graphtype \$[12],Margins \$ (2)[10],Papersize\$[1]

COM /Background/ REAL Pen_speed,INTEGER Backgnd_pen,Auto_time

COM /Background/ INTEGER Auto_file,REAL X_cross_y,Y_cross_x

COM /Background/ Xgrid_tick\$[4],INTEGER Xmajor,Xminö

COM /Background/ Ygrid_tick\$ [4],INTEGER Ymajor,Yminor

COM /Background/ REAL Xmin_graph,Xmax_graph,Ymin_graph,Ymax_graph

236

238

240

242

244

246

248

250

252

254

COM /Bugs/ INTEGER Bug1,Bug2,Bug3,Printer

COM Anterrupts/ INTEGER Intr_prty

COM /Enlarge_file/ INTEGER Overflow

COM /Files/ Diskdrive $\$[20]$, Filename $\$[141, M s$ path $\$[500]$

COM /Data_stuff/ INTEGER Number,REAL Delta_x,REAL Strt_time

256

258

260

262

264

266

INTEGER R,Hold_size,Local_prty,Allocated,Fls_cnt

DIM Ac \$[5],Temprile $\$[10]$, Mask \$[10],Ftype $\$[5], F \mid s \$(1)[10]$

REAL Dtime

OFF KEY

Local_prty = Intr_prty

1

ISelect the disk drive where the data exists

1

IF Overflow $<>0$ THEN Overflow $=0$

Hold_size $=0$

Dtime $=0$.

268 Selectdrive:

270 IF Diskdrive $\$=$ "NO DISK" THEN Diskdrive $\$="$ "

272 IF LEN(Diskdrive $\$$ ) >0 THEN GOTO Choosefilename

274 GRAPHICS OFF

276 OUTPUT 2 USING "\#,K";"K"

278 CALL Select_disk

280 IF Diskdrive $\$$ = "NO DISK" THEN GOTO Mistakelineset

282 Choosefilename: I

284

286

288

290

292

294

296

298

300

302

304

306

308

310

Tempfile $\$=$ Filename $\$$

IF LEN(Filename\$ $>0$ THEN GOTO Bring_in_data

AC $\$=$ "CAT"

CALL Enterfilename(AC\$)

IF LEN(Filename $\$$ ) $=0$ OR POS(Filename $\$, "$ " ") $>1$ THEN

IF POS(Filename\$," ") > 1 THEN \& set mask\$ Mask $\$=$ Filename $\$[1, P O S(F i l e n a m e \$, " *)-1]$

Filename $\$=n$

ELSE

$$
\text { Mask } \$=" \text { I no preselection }
$$

END IF

Ftype $\$=$ "ASCII" I examine ASCII files only

Fls_cnt $=1$ | select one file

Intr_prty = Local_prty +1

CALL File_menu(Mask $\$, F$ type $\$, F 1 s \$\left(0^{*}\right)$,Fls_cnt, 0,0$)$

Intr_prty $=$ Local_prty

Fileñame $\$=$ FIs $\$(q)$

IF LEN(Filename $\$$ ) $=0$ THEN ! aborted

Filename $\$=$ Tempfile $\$$

GOTO Mistakelineset

END IF 
END IF

328 Bring_in_data: I

332 Ifind this file on the disk.

$334 \quad 1$

336 ON ERROR GOTO Cant_findfile

338 ASSIGN @Datapath TO Filename \$\&Diskdrive\$

340 OFF ERROR

342 Dtime $=$ TIMEDATE

344 DISP " LOADING disk file: ";Filename $\$ ; " . . . "$ ";

346 ON ERROR GOTO Bad_file

348 ENTER @Datapath;Status\$

350 OFF ERROR

352

354

ON ERROR GOTO Cant_findfile

SELECT StatUS\$

356

358

360

362

364

366

368

370

372

374

376

378

380

382

384

386

388

390 Bad_file: DISP CHR\$(12)

392

394

396

398

400

402

404

406

408

410

412

414

416

418

420

422

424

426

428

430

432

434

436

CASE "Y" I All graphics/data parameters exist.REN 100,2

DISP " Complete graph."

ENTER @Datapath;Time_orgn\$,Date_orgn\$

ENTER @Datapath;Time_chng\$,Date_chng\$

ENTER @Datapath;Description\$

ENTER @Datapath;Labels $\$(*), L b \mid$ count,Lbl_addr(*)

ENTER @Datapath;Curve_id $\left.\$(*), \overline{S y m b o l} \$ 1^{*}\right)$

ENTER @Datapath;Roster("),Curvecount

ENTER @Datapath;Graphtype \$,Margins $\$(*)$

ENTER @Datapath;X_cross_y,Y_cross_X

ENTER @Datapath;Xgrid_tick\$, Xmajor,Xminor

ENTER @Datapath;Ygrid_tick\$,Ymajor,Yminor

ENTER @Datapath;Xmin_graph,Xmax_graph

ENTER @Datapath;Ymin_graph,Ymax_graph

CASE " $N$ " I Only data parameters exist.

DISP " RAW data."

CASE ELSE

DISP "Data file is not recognized, entry aborted.";

DISP " ...continue."

BEEP

PAUSE

OFF ERROR

GOTO Mistakelineset

END SELECT

!

ENTER @Datapath;Data_id\$

IF FIg THEN

ENTER @Datapath;Delta_x

ENTER @Datapath;Datacount

ELSE Hold_size = Datacount

ENTER @Datapath;Datacount

ENTER @Datapath;Hold_size

END IF

IF NOT Allocated THEN

IF Datacount $>=1$ AND Hold_size $>=1$ THEN

ALLOCATE Holding_file(Hold_size, 2)

ELSE

ALLOCATE Holding_file $(1,2)$

END IF 


$$
\text { Allocated }=1
$$

494 Copydatafile: ENTER @Datapath;Holding_file(") ASSIGN @Datapath TO. OFF ERROR

510 Mistakelineset:Datacount $=0$

512 Mistakeline:OFF KEY

514 IF Allocated THEN DEALLOCATE Holding_file $(\%)$

596 LOOP

518 EXIT IF TIMEDATE-Dtime $>9.8$

520 END LOOP

522 DISP CHR\$(12)

524 OUTPUT 2 USING ${ }^{\circledR}{ }^{\circ} \mathrm{K}^{\mathrm{*}}{ }^{\circ}{ }^{\circ} \mathrm{K}^{\circ}$

526 SUBEXIT

$528 \quad 1$

$530 \quad$ I IIIIIIIIIIIIIIIIIIIIIIIIIIIIIIIIIIIIIIIIIIIIIIIIIIIII

532

534 Cant findfile: IError in searching for the file. 


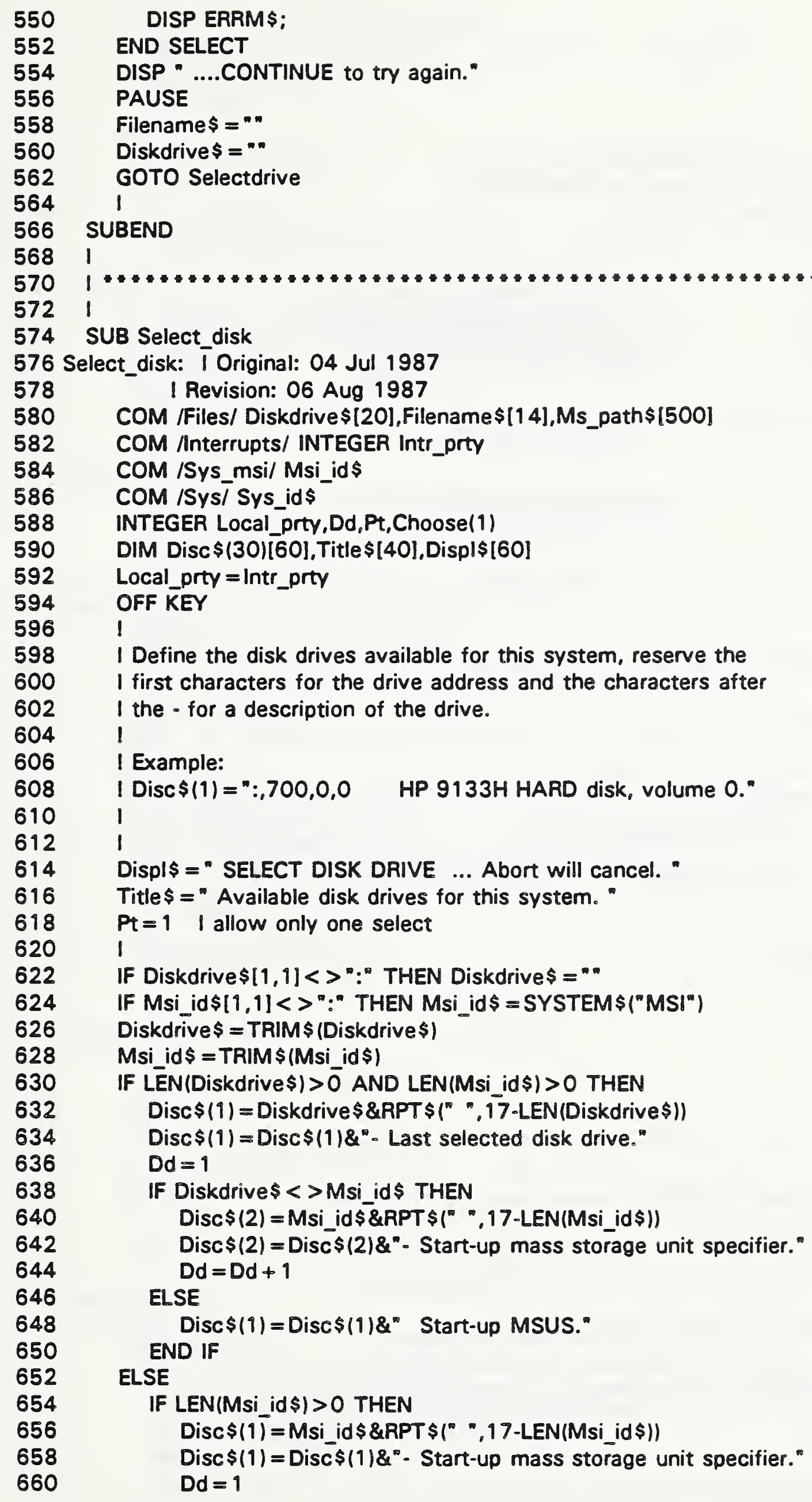

5721

574 SUB Select_disk

576 Select_disk: T Original: 04 Jul 1987

578 I Revision: 06 Aug 1987

580 COM /Files/ Diskdrive $\$[20]$,Filename $\$[14], M s \_p a t h \$[500]$

582 COM /nterrupts/ INTEGER Intr_prty

584 COM /Sys_msi/Msi_id\$

586 COM /Sys/ Sys_id\$

588 INTEGER Local_prty,Dd,Pt,Choose(1)

590 DIM Disc $\$(30)[60], T i t l e \$[40]$, Displ\$[60]

592 Local_prty = Intr_prty

594 OFF KEY

$596 \quad 1$

598

600

602

604

606

608

610 1

I Define the disk drives available for this system, reserve the I first characters for the drive address and the characters after I the - for a description of the drive. 


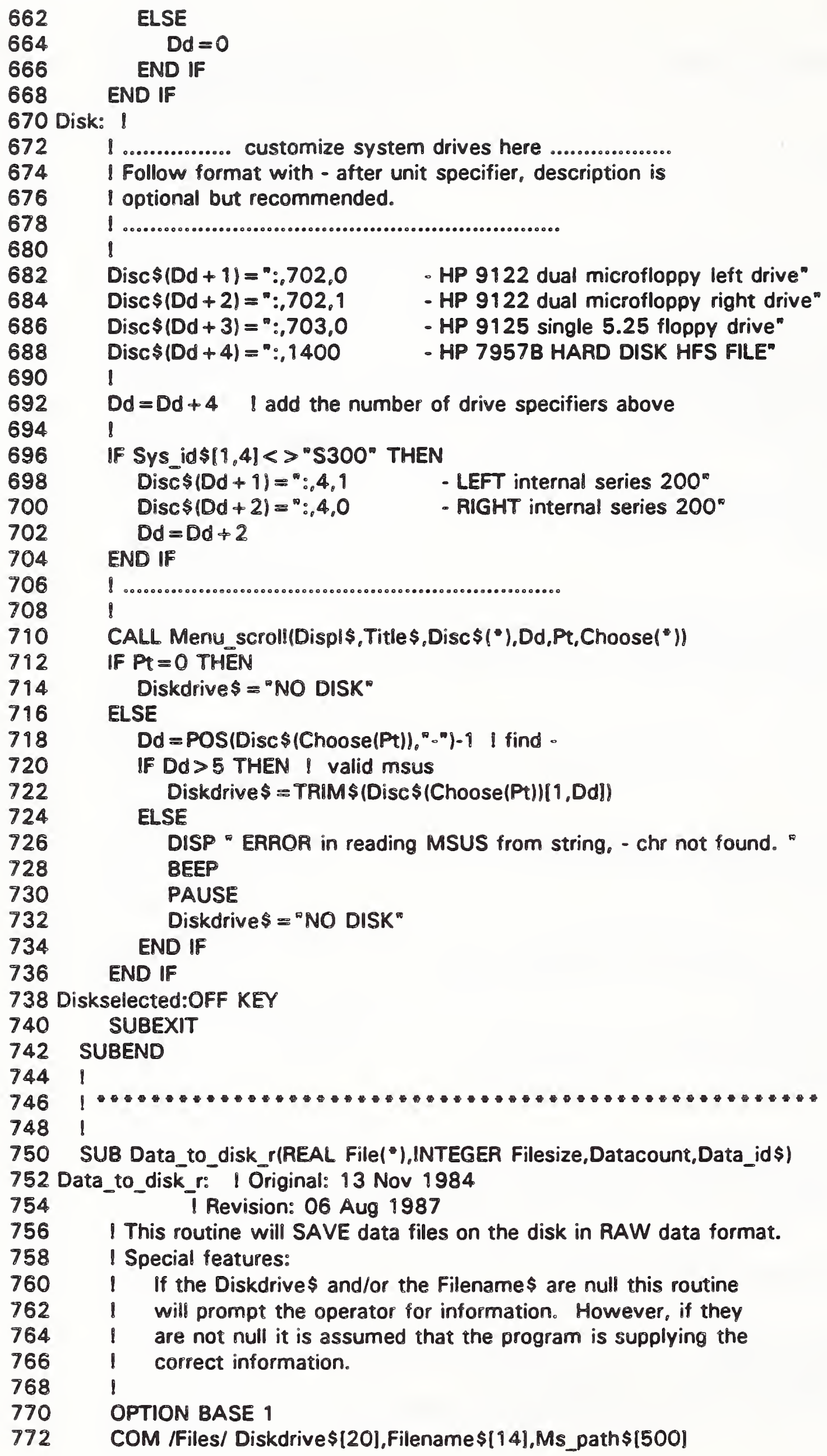


776 INTEGER Local_prty,Diskspace

778 DIM Ac $\$[5]$, Status $\$[1]$

780 REAL Dtime

782 OFF KEY

784 Local_prty = Intr_prty

786 Dtime $=0$.

$788 \quad 1$

794 Selectdrive: 1

796 IF Diskdrive $\$=$ "NO DISK" THEN Diskdrive $\$="$ "

798 IF LEN(Diskdrive $\$$ ) >0 THEN GOTO Choosefilename

800 GRAPHICS OFF

802 OUTPUT 2 USING "\#,K";"K"

804 CALL Select disk

806 IF Diskdrive $\$=$ "NO DISK" THEN GOTO Mistakeline

808 Choosefilename: 1

890 IF LEN(Filename $\$$ ) >0 THEN GOTO Send to_disk

812 AC\$ = "ABORT"

814 CALL Enterfilename(Ac\$)

816 IF LEN(Filename $\$$ ) $=0$ THEN GOTO Mistakeline

818 Send to disk: ! Create file and save information.

820 ON ERROR GOTO Cant savedata

822 Diskspace $=$ INT $(($ Filesize $*$ 16.0)/256) +2

824 CREATE ASCII Filename\$\&Diskdrive\$,Diskspace

826 Dtime $=$ TIMEDATE

828 DISP " SAVING data in file ";Filenames;" on ";Diskdrive\$

830 Status $\$=$ " $N "$

832 ASSIGN @Datapath TO Filename\$\&Diskdrive\$

834 OUTPUT @Datapath;Status\$

836 OUTPUT @Datapath;Data_id\$ $140 \mathrm{chrs}$ description of data

838 OUTPUT @Datapath;Datacount Inumber of xy points

840 OUTPUT @Datapath;Filesize Isize of array

842 OUTPUT @Datapath;File(")

844 ASSIGN @Datapath TO.

846 OFF ERROR

$848 \quad 1$

850 Mistakeline:OFF KEY

852 LOOP

854 EXIT IF TIMEDATE-Dtime $>1.8$

856 END LOOP

858 DISP CHR\$ 1121

860 OUTPUT 2 USING "\#, $K^{m} ; "{ }^{\circ} K^{*}$

862 SUBEXIT

864 !

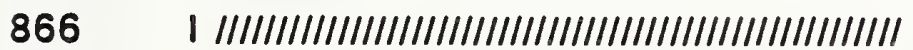

$868 \quad !$

870 Cant_savedata: !

872 BEEP 500.6

874 SELECT ERRN

876 CASE $72,73,76,78,81,82,90,93$

878

880

882

DISP Diskdrive\$;" has failed or is not available ";

DISP " ....CONTINUE to try again."

884 Diskdrive $\$=$ " 


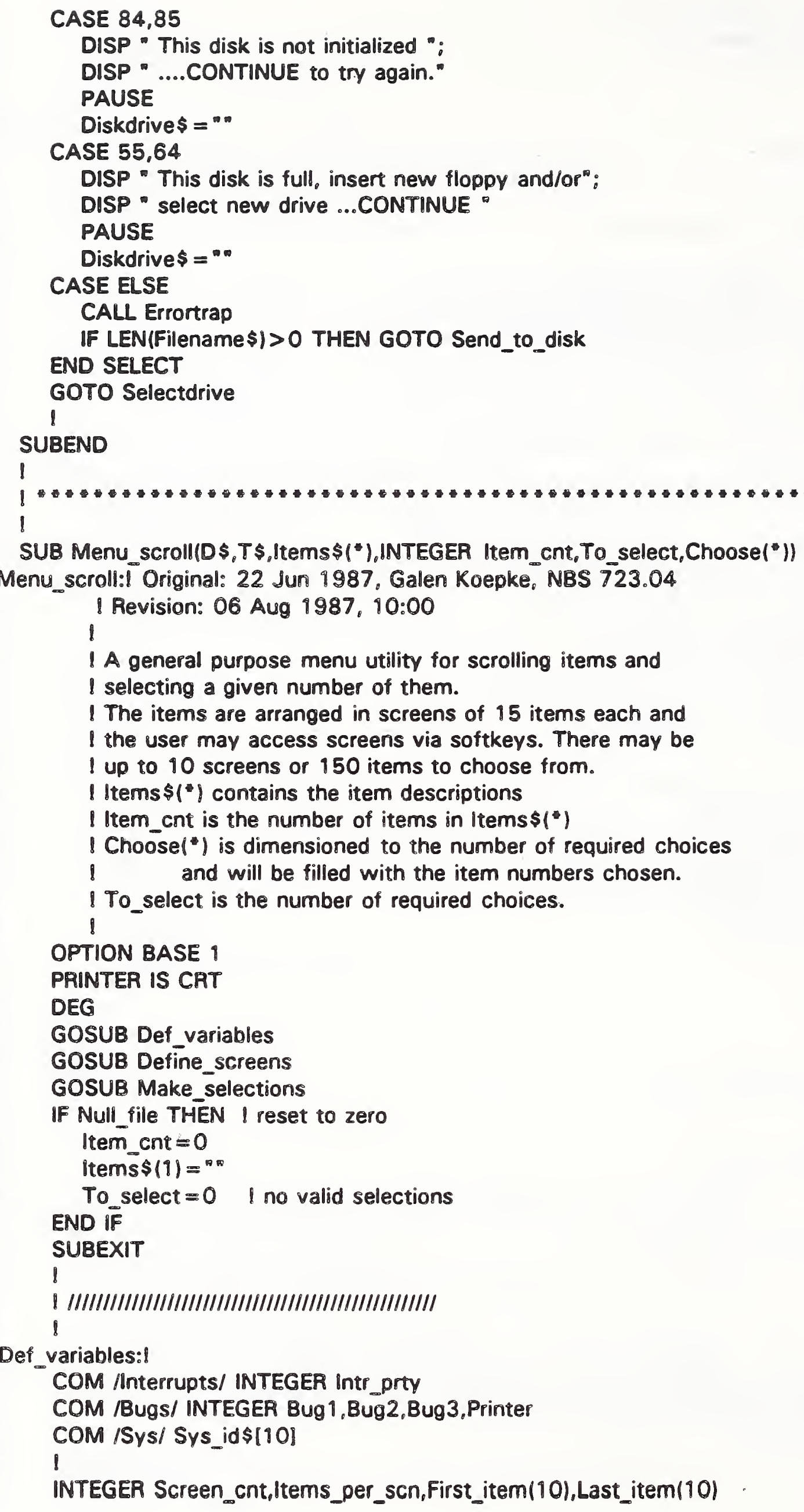



INTEGER Local_prty,Skips,Kñobcount,Pointeractive,KO,Null_file INTEGER Exit flag DIM Marker\$[8],Test\$[160]

1052 Define screens:। Set up screens of 15 items each.

1096 Make selections:I MENU setup and use.

1098 Active_screen $=1$ I first screen is active

1100 First_line $=2 \quad$ I first printed line on screen $=2$ or greater. IF INT(Item_cnt/ltems_per_scn) =Item_cnt/ltems_per_scn THEN ELSE Screen_cnt $=$ INT (Item_cnt/ltems_per_scn)

\section{END IF}

$J=1$

FOR $\mid=1$ TO Screen_cnt $\mid$ set up each screen

First_item $(I)=\mathrm{J}$

IF J + Items_per_scn-1 <Item_cnt THEN

Last_item $(I) \equiv J+$ Items_per_scn-1 $\mathrm{J}=\mathrm{J}+$ Items_per_scn

ELSE

END IF

NEXT I

RETURN

I

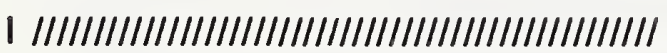

$$
1
$$


$1910 \quad$ Exit_flag $=0 \quad$ I allow ENTER key to exit when selections filled.

1112 IF Sys_id $\$ 1,4]=$ "S300" THEN

1114

1916

9118

1120

1122

1124

1126

1128

1930

1132

1134 Key_loop: I

1138

1140

1942

1144

9146

1948

1950

$\$ 152$ CONTROL KBD, $2 ; 1$

STATUS KBD, $14 ; J$

IF $J=0$ THEN $\mid$ key 1 defined $K O=1$

ELSE I key $O$ defined

$\mathrm{KO}=\mathrm{O}$

END IF

ELSE

$K O=0$

END IF

ON KBD,Local_prty GOSUB Process_kbd

ON KNOB .01,Local_prty GOSUB Move_pointer

IF Skips < TO_select THEN

DISP DS

IF TO_select $>1$ THEN

Test $\$=$ " Select "\&VAL\$(Skips $+11 \&$ " of "\&VAL\$(To_select)

ELSE

Test $\$={ }^{*}$ Select ${ }^{*}$

END IF

9154

1156

1958

1160

1962

1964

9166

1968

1170

9172

1174

9176

ON KEY KO LABEL Test\$,Local_prty GOSUB Select_item

ELSE

IF To select $>0$ THEN

ELSE

DISP " Selection process complete ..."

DISP " Menu for information only ..."

END IF

END IF

ON KEY KO LABEL "Accept",Local_prty GOTO Exit_line

IF Active_screen < Screen_cnt THEN

ON KEYY KO + 1 LABEL" "Next Screen",Local_prty GOSUB Next_screen

1978

1180

1182

1184

1186

1188

1190

1992

1194

1196

1198

1200

ELSE

OFF KEY KO + 9

END IF

If Active_screen $>1$ THEN

ON KEY KO + 2 LABEL " Last Screen" Local_prty GOSUB Last_screen

ELSE

OFF KEY KO +2

END IF

IF Skips $>0$ THEN

ELSE

ON KEY KO+ 3 LABEL " Reset Select" "Local_prty GOSUB Select_reset

$$
\text { OFF KEY KO }+3
$$

END IF

1202

1204

1206

1208

1210

1212

1214

1216 Escape line:Skips $=0$

1218 MAT Choose $=(0)$

1220

IF To_select $>0$ THEN

ON KEY KO + 4 LABEL " Abort "Local_prty GOTO Escape_line ELSE

OFF KEY KO +4

END IF

IF Exit_flag THEN Exit_line

GOTO Key_loop

To_select $=0$ 
1222 Exit_line:OFF KEY

1224 OFF KNOB

1226

OFF KBD

1228

1230

1232

1234

1236

1238

1240

1242 Next

1244

1246

1248

OUTPUT KBD;CHR\$(255)\&CHR\$(75);

PRINT CHR\$(128);

I everything cleared, now go back to work.

RETURN

1

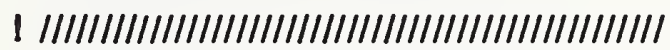

1

OFF KBD

OFF KNOB

OFF KEY

1250

IF Active_screen $=$ Screen_cnt THEN RETURN

1252

1254

1256

1258

1260

1262

1264

1266

1268

1270

1272

1274

1276

1278 Active_screen $=$ Active_screen +1

GOSUB Write_screen

RETURN

1

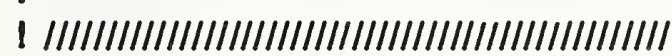

1

Last_screen:

OFF KBD

OFF KNOB

OFF KEY

IF Active_screen $=1$ THEN RETURN

Active_screen $=$ Active_screen-1

GOSUB Write_screen

1280

1282

1284

1286 Select_item:!

1288 OFFF KBD

1290 OFF KNOB

1292 OFF KEY

1294 IF NOT Pointeractive THEN

1296

1298

1300

1302

1304

1306

1308

1310

1312

1314

1316

1318

1320

1322

1324

1326

1328

1330

1332

RETURN

I

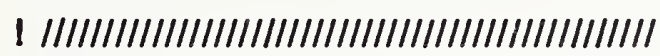

DISP "NO additional selections for this screen."

BEEP

WAIT 2

DISP CHRS (12):

RETUAN

END IF

If Skips $=$ To_select THEN

IF To_select $=0$ THEN

DISP "This menu is for information only,":

DISP " no selection allowed."

ELSE

DISP "All selections have been filled,":

DISP " 'Select Reset' to repeat."

END IF

BEEP

WAIT 2

DISP CHR\$(12):

RETURN

END IF 
1402

1404

1406

1408

1410 Select

1412

1414

1416

1418

1420

1422

1424

1426

9428

1430

1432

1434

1436

1438

1440

1442

1444

I

I

Skips $=$ Skips +1

Choose(Skips) $=$ First_item (Active_screen) + Pointer-First_line

PRINT CHRS(129): I inverse video

PRINT TABXY(10,Pointer);Items $\$$ (Choose(Skips))

PRINT CHR\$(1 28):

PRINT TABXY(1,Pointer):

SELECT Pointer

CASE First line GOSUB Point_forward

CASE Last_line GOSUB Point_backward

CASE ELSE

I move forward unless it requires wrapping to beginning.

IF Skips-1 >0 THEN I check for selected items.

$I=$ Pointer-First_line

LOOP

$$
K=0
$$

FOR $J=1$ TO Skips

If First_item(Active_screen) $+1=$ Choose(J) THEN $K=1$ NEXT $d$

EXIT IF $K=0$

$1=1+1$

IF $1+$ First line $>$ Last line THEN $K=-1$

EXIT IF $K=-\vec{q}$

END LOOP

IF $K=0$ THEN

GOSUB Point_forward

ELSE

GOSUB Point_backward

ELSE

END IF

GOSUB Point_forward

END IF

END SELECT

RETURN

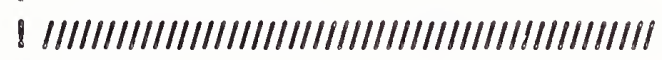

1

IClear Choose file

OFF KBD

OFF KNOB

OFF KEY

Skips $=0$

MAT Choose $=(0)$

GOSUB Write_screen

RETURN

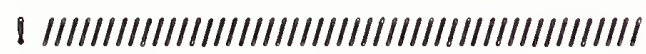

1

Test $\$=$ KBD $\$$

IF LEN(Test\$) $=1$ AND Tes $\{[1,1]<>$ CHR\$(32) THEN

BEEP $80 . .09$

RETURN

END IF

IF Test $\$\{, 1\}=$ CHR $\$(32)$ THEN GOSUB Point_forward 
IF Test $\$[1,1]<>$ CHR\$(255) THEN RETURN

1448

1450

1452

1454

9456

1458

1460

1462

1464

1466

1468

1470

1472

1474

1476

1478

1480

1482

1484

1486

1488

1490

1492 Point

SELECT Test $\$ 2,2]$

CASE CHR\$(255)

CASE " $V$ ", "T"

I do nothing

GOSUB Point_forward

CASE "A" " "W"

GOSUB Point_backward

CASE "E"

IF Skips <To_select THEN

GOSUB Sélect_item

\section{ELSE}

1 exit routine

Exit_flag $=1$

END !F

CASE ELSE

BEEP $80 . . .1$

END SELECT

Test $\$=" m$

RETURN

1

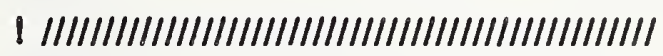
1

Point forward: Knobcount $=5$

1494 ḠOSUB Move_pointer

1496 RETURN

1498 Point_backward:Knobcount $=-5$

1500 ḠOSUB Move_pointer

1502 RETURN

1504 I

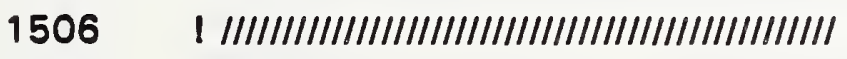

1508

1510 Jog_pointer:l Move the selection pointer on the active screen.

1512

1514

1516

1518

1520

1522

1524

1526

1528

1530

1532

1534

1536 Move_pointer:! Control pointer to avoid re-selection of items

1538 IF NOT Pointeractive THEN RETURN I No selections to be made.

1540 Knobcount $=$ Knobcount + KNOBX + KNOBY

1542 IF ABS(Knobcount) <4 THEN RETURN

1544

1546

1548

1550

1552

1554

1556

Last_pt $=$ Pointer

GOSUB Jog_pointer

IF Skips $>0$ THEN

LOOP

$J=$ Pointer-First_line

FOR $I=1$ TO Skips

If First_item(Active_screen) $+\mathrm{J}=$ Choose(l) THEN $\mathrm{J}=999$ 
1586

1588

1590

1592

1594

1596

1598

1600

1602

1604 Write_screen:I Write the screen pointed to by Active_screen

1606

1608

1610

1612

1614

1616

1618

1620

1622

1624

1626

1628

1630

1632

1634

1636

1638

1640

1642

1644

1646

1648

1650

1652

1654

1656

1658

1660

1662

1664

1666

1668

NEXT I

IF $\mathrm{J}=999$ AND Pointer $=$ Last_pt THEN Pointeractive $=0$

EXIT IF Pointeractive $=0$

IF $J=999$ THEN GOSUB Jog_pointer

EXIT IF $\mathrm{J}<>999$

END LOOP

END IF

Knobcount $=0$

OUTPUT KBD;CHR\$(255)\&CHR\$(84): I Bring screen home

IF Last_pt = Last_line THEN PRINT CHR\$(132):

PRINT ":

IF Pointeractive THEN \& Pointer active

IF Pointer = Last line THEN PRINT CHR\$ 1132$)$ :

ELSE

PRINT CHR\$ (128):

END IF

PRINT TABXY(1,Pointer);Marker\$;CHR\$(128):

END IF

RETURN

1

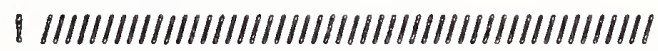

I home and clear screen

OUTPUT KBD;CHR\$(255)\&CHR\$(84)\&CHR\$(255)\&CHR\$175):

Knobcount $=K N O B X+K N O B Y \quad \&$ Clear knob and keyboard

Knobcount $=0$

Test $\$=$ KBD $\$$

Test $\$=" \infty$

!

PRINT TABXY(1, First line-1):CHR\$(132):" Item \# Screen \#":

PRINT USING "\#,2D, $3 A_{0}, 2 D, 3 A^{*}$;Active_screen; ${ }^{*}$. of ";Screen_cnt;" $\left.\right|^{\text {" }}$ PRINT T\$;APT \$ ${ }^{\circ}{ }^{\circ}, 52-L E N(T \$) ; C H R \$(128) ;$

$J=0$

REPEAT

IF $J=$ Last item(Active_screen)-First item(Active_screen) THEN PRINT CHR\$ (132):

PRINT TABXY(?,First line + J):RPT $\$\left(^{\circ} "{ }^{\circ}, 80\right)$

ELSE

PRINT CHR\$(128):

END IF

PRINT TABXY $(5$, First_line $+J)$;

PRINT USING " $30, A, \#^{*}$; First item(Active screen) $+\left.J_{0}{ }^{\circ}\right|^{\circ}$

If Skips $>0$ THEN I make this line inverse video

FOR $I=1$ TO Skips

IF First item(Active_screen) $+J=$ Choose(I) THEN PRINT CHR\$(129);

END IF

NEXT I

END IF

PRINT TABXY(10,First_line $+J)$ :ltems $\$$ (First_item(Active_screen) $\rightarrow J$ )

$J=J+1$

UNTIL $J>=$ (Last_item(Active_screen)-First_item(Active_screen) +1 )

Last_line $=$ Last_item (Active_screen)-First_item(Active_screen)

Last_line = Last_line + First_line 


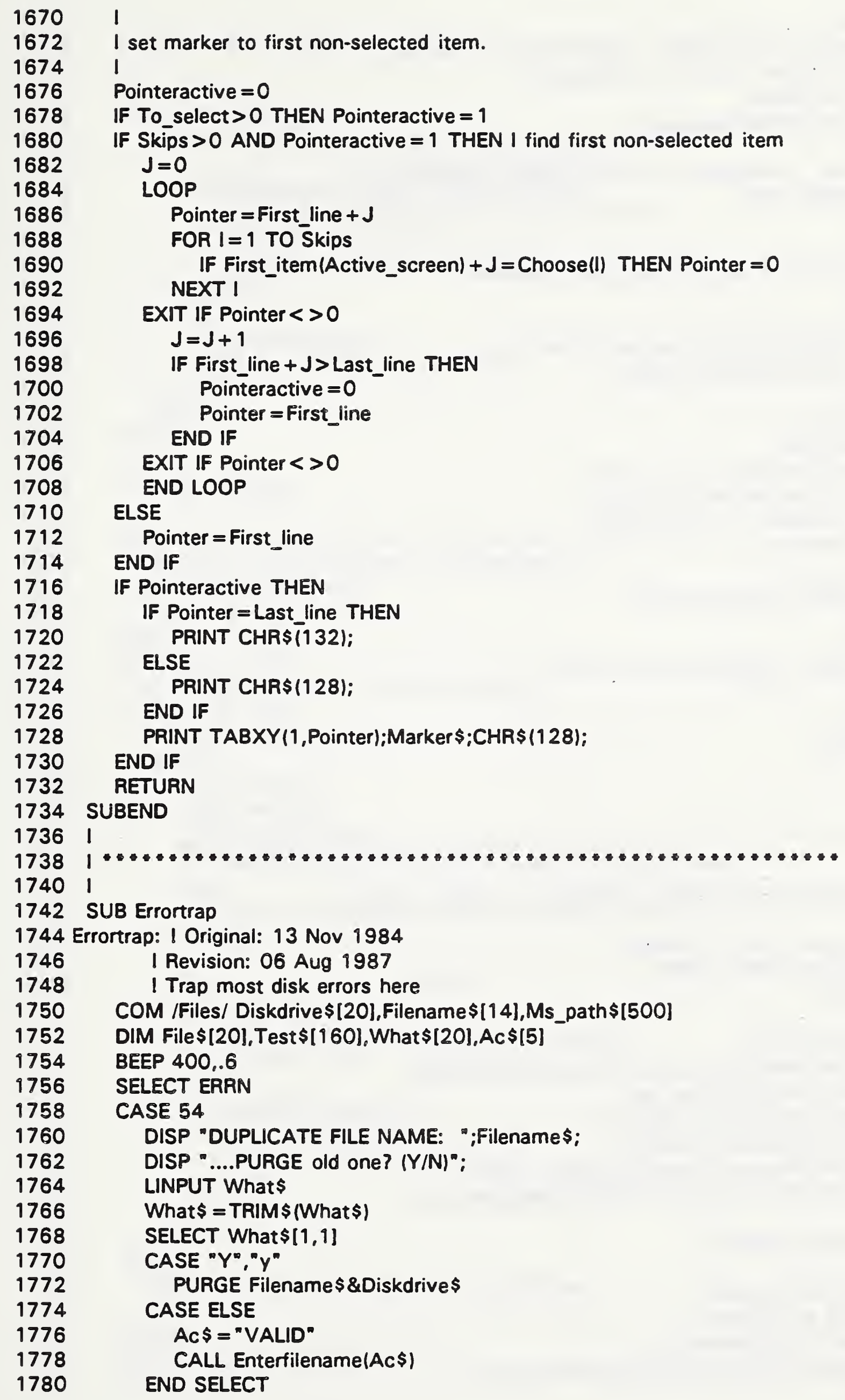


1862

1864

1866

9868

1870

1872

1874

1876

1878

1880

1882

1884

1886

1888

1890

1892

\section{CASE 52,53}

DISP "Improper FILE NAME - ENTER NEW FILE NAME";

OUTPUT 2 USING "\#,K,K";" \#";Filename\$

LINPUT Filename\$

Filename $\$=$ TRIM $\$$ (Filename $\$$ )

CASE 56

DISP "FILE: ":Filename $\$ "$ is not on this disk, please insert":

DISP " correct disk"

PAUSE

\section{CASE 64}

DISP "This disk is full, PLEASE insert clean disk"

PAUSE

CASE 56

DISP "DATA INPUT disk must be in drive!! ": DISP "...CONTINUE when ready."

PAUSE

\section{CASE 72,73,76}

DISP Diskdrive\$:

DISP " is not available type correct";

DISP " unit specifier (ie. $:, 707,0 \%$ ).":

OUTPUT 2 USING "K,\#":Diskdrive\$

LINPUT Diskdrive\$

\section{CASE 80}

DISP "CHECK DISK drive door!"

PAUSE

CASE ELSE

DISP ERRM\$;" 'CONTINUE' when fixed ${ }^{\circ}$

PAUSE

END SELECT

DISP CHR\$(12)

SUBEXIT

SUBEND

1

1

SUB File_menu(Mask\$,Ftype\$,FIs\$("),INTEGER FIs_ent,Dir_on,Prton)

le_menu: !

I Original: 29 Jun 1987, G. Koepke

I Revision: 06 Aug 1987, 10:00

OPTION BASE 1

DEG

COM /Sys/ Sys id \$ [10]

COM /Files/ Diskdrive $\$[20]$,Filename $\$[14$ ],Ms_path $\$[500]$

COM /Interrupts/ INTEGER Intr_prty

DIM Directory $\$(150)[80], \mathrm{Bd} \$(150)[71]$

DIM D \$[80],T \$[52],Ids\$[40],Stat\$[1]

INTEGER Bd_cnt,File_cnt, I,C_ent,CO(1),Format_error

IF Fls_cnt $>0$ THEN ALLOCATTE INTEGER Choose(FIs_cnt) I

I Catalog the disk specified 1

ON ERROR GOTO Cat_errors

DISP " Reading the Directory ..."

MASS STORAGE IS Diskdrive $\$$

CAT TO Directory \$("):NO HEADER,COUNT File_cnt OFF ERROR 


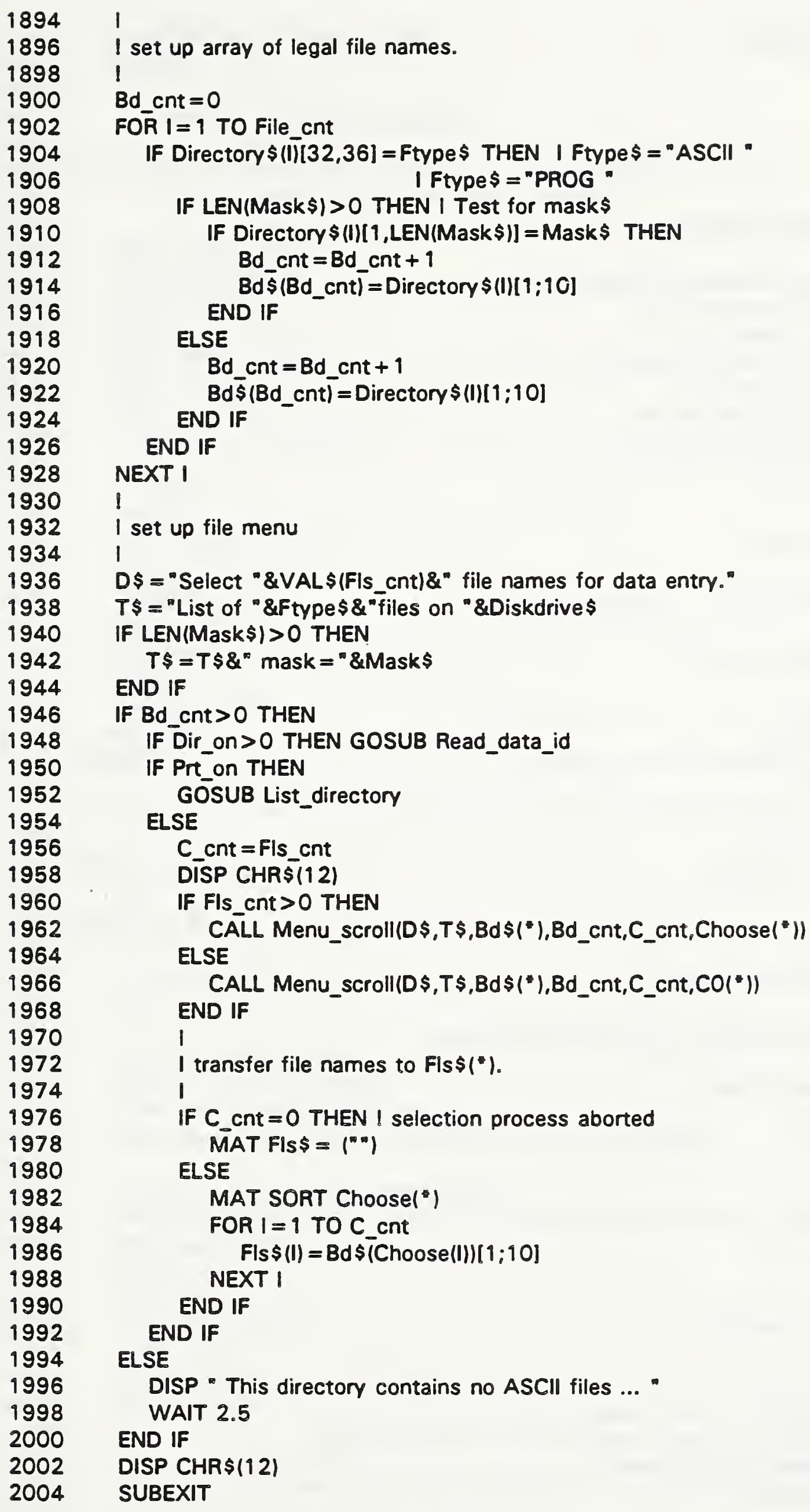

1924

1926 
2006 Cat_errors:!

2008 DISP "ERROR ... ";ERRM\$

2010 BEEP

2012 PAUSE

2014 C_cnt $=0$

2016 MAT FIS $\$=(\%)$

2018 SUBEXIT

$2020 \quad 1$

2022

2024

2026 Read_data_id: I This routine expects to see Ids $\$$ from

2028

2030

2032

2034

2036

2038

2040

2042

2044

2046

2048

2050

2052

2054 Not_recognized:ASSIGN @lo_path TO.

2056

2058

2060

2062

2064

2066

2068

2070

2072

2074

2076

2078

2080

2082 Interpret_ $1: 1$ This is used to interpret TEM program ID strings.

2084 Format_error $=0$

2086 I identify this particular format

2088 IF LEN(Ids $\$$ ) $<40$ THEN

2090

2092

2094

2096

2098

2100

2102

2104

2106

2108

2910

2912

2914

END IF

OFF ERROR

IF Dir_on $=2$ THEN

GOSUB Interpret_1

IF Format_error THEN GOTO Other format

GOTO Go on

Other format:!

Go_on:NEXT I

RETURN

1

I IIIIIIIIIIIIIIIIIIIIIIIIIIIIIIIIIIIIIIIIIIIIIII

$$
\text { Format_error }=1
$$

END IF

If Ids $\$[40]<>$ THEN

Format_error $=1$

RETURN

END IF

I make the information readable

$\mathrm{Bd} \$(1)[19,15]=\ldots$

$\operatorname{Bd} \$(1)[96,25]=1 d s \$[1,90\}$

$\operatorname{Bd} \$(1)[26,32]={ }^{*}$ " \&lds $\$[11,12] \& ":$ : \&lds $\$[13,14]$

$\mathrm{Bd} \$(1)[33,42]={ }^{\circ}$ " \&lds\$[15,16]\&" " \&lds\$[17,18]\&" " \&lds\$[19,20]

$\operatorname{Bd} \$(1)[43,55]="$ " "\&lds $\$[21,27] \& " \mathrm{MHz}^{\mathrm{N}}$

$2116 \quad B d \$(1)[56,65]=", "$ "\&lds $\$\left[28,331 \&^{\prime \prime} \mathrm{vm}^{\prime \prime}\right.$ 
2180 SUB Read_data

2182 !

2984 Read_data: !

2186 !

2188

2190

2192

2194

2196

2198 
IF Overflow $<>0$ THEN Overflow $=0$

Hold_size $=0$

Dtime $=0$.

2236 Allocated $=0$

2238 Selectdrive:

2240 IF Diskdrive $\$=$ "NO DISK" THEN Diskdrive $\$=$ "

2242 IF LEN(Diskdrive \$) >0 THEN GOTO Choosefilename

2244 GRAPHICS OFF

2246 OUTPUT 2 USING "\#,K";"K"

2248 CALL Select_disk

2250 IF Diskdrive \$ = "NO DISK" THEN GOTO Mistakelineset

2252 Choosefilename: 1

2254 Tempfile $\$=$ Filename $\$$

2256 IF LEN(Filename\$) $>0$ THEN GOTO Bring_in_data

2258 AC\$ $=$ "CAT"

CALL Enterfilename(AC\$)

2262

IF LEN(Filename $\$$ ) $=0$ OR POS(Filename $\$, "$ ") $>1$ THEN

IF POS(Filename $\$, " *$ ") $>1$ THEN I set mask $\$$ Mask $\$=$ Filename $\$[1, P O S(F i l e n a m e \$, " *$ " $)-1]$ Filename $\$=" m$

\section{ELSE} Mask $\$="$ I 1 no preselection

\section{END IF}

Ftype $\$=$ "ASCII" I examine ASCil files only

Fis_cnt $=1$ | select one file

Intr_prty = Local_prty +1

CALL File_menu(Mask $\left.\$, F t y p e \$, F i s \$(*), F i s \_C n t_{0}, 0,0\right)$

Intr_prty $=$ Local_prty

Filename $\$=$ Fls $\$(\eta)$

IF LEN(Filename $\$$ ) $=0$ THEN $\mid$ aborted

Filename $\$=$ Tempfile $\$$

GOTO Mistakelineset

\section{END IF}

END IF

Ifind this file on the disk.

\&

ON ERROR GOTO Cant findfile

ASSIGN @Datapath TO Filename $\$$ \&Diskdrive $\$$

OFF ERROR

Dtime $=$ TIMEDATE

DISP " LOADING disk file: ";Filename $\$_{;}^{* \prime . . . " ~}$

ENTER @Datapath;Data_id\$

1

ENTER @Datapath;Datacount

IF NOT Allocated THEN

IF Datacount $>=1$ THEN

ALLOCATE Holding_file $\$$ (Datacount)[80]

ELSE

ALLOCATE Holding_file $\$(1)[80]$

END IF

Allocated $=1$

END IF

ENTER @Datapath; Holding_file $\$($ )

ASSIGN @Datapath TO . 
OFF ERROR

2344 IF Datacount $=0$ THEN Mistakeline

2346 PRINTER IS PRT

2348

2350

2352

2354

2356

2358

2360

2362

2364 Mistakelineset: Datacount $=0$

2366 Mistakeline:OFF KEY

2368! IF Allocated THEN DEALLOCATE Holding_file $\$(")$

2370 LOOP

2372 EXIT IF TIMEDATE-Dtime > 1.8

2374

2376

2378

2380

2382

2384

2386

2388

2390

2392

2394

2396

2398

2400

2402

2404

2406

2408

2410

2412

2414

2416

2418

2420

2422 !

2424

2426 !

2428

2430 !

2432 SUB Enterfilename(AC\$)

2434 Enterfilename: 1 Original: 13 Nov 1984

2436

2438

2440

2442

2444

2446

2448

END LOOP

DISP CHR\$(12)

OUTPUT 2 USING "\#,K";"K"

SUBEXIT

1

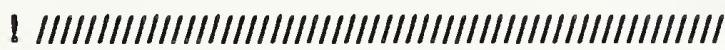

1

IError in searching for the file.

BEEP 500,.6

SELECT ERRN

CASE 56

DISP "That file does not exist on this disk ":

CASE $72,73,76,82$

DISP Diskdrives;" has failed or is not available ";

CASE ELSE

DISP ERRMS;

END SELECT

DISP " ....CONTINUE to try again."

PAUSE

Filename $\$=m$ *

Diskdrive $\$="$ "

GOTO Selectdrive

I

RETURN

SUBEND

2450

2452

I Revision: 10 Dec 1990 includes HFS directories

OPTION BASE 1

COM /Files/ Diskdrive \$[20],Filename \$[14],Ms_path \$[500]

COM /nterrupts/ INTEGER Intr_prty

INTEGER I,Ascii_num,Maskflag,Namelength

DIM Test\$[256],Hfs_temp\$[161]

Namelength $=10$

IF LEN(Ms_path\$) >0 THEN OUTPUT KBD USING "K, $\#^{n} ;^{n} \#^{n} \& M s \_p a t h \$ \&{ }^{n} H^{n}$

DISP " ENTER HFS directory PATH (no file)"; 
IF AC\$<> "PATH" THEN

DISP ", ENTER / for HFS ROOT or null for LIF...":

END IF

LINPUT HFs temp\$

His temp $\$=$ TRIM $\$$ (Hfs temp $\$$ )

IF LEN(His temp $\$$ ) $>0$ THEN

IF LEN(Hifs_temp\$) 1 AND His_temp\$[LEN(Hfs_temp $\$ ;: 1]<>\pi$ THEN His_temp $\$=$ Hfs_temp $\$ \&^{m} /{ }^{\circ}$ END IF IF LEN(Hfs_temp\$) $=1$ THEN Hfs_temp $\$=m$ Namelength $=14$

END IF

IF AC $\$=$ "PATH" THEN

Ms_path\$ $=$ Hfs_temp\$

SUB̈EXIT

END IF

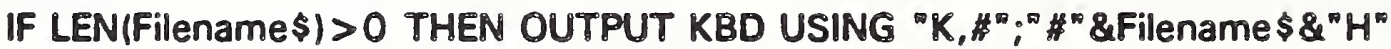

DISP " ENTER the FILE NAME ...":

SELECT AC\$

CASE "CAT"

DISP "(ENTER CAT mask or ENTER null to CAT)"

CASE "ABORT"

DISP " (ENTER null to ABORT)":

CASE "VALID"

DISP "(must be a VALID name!) ":

END SELECT

LINPUT Test\$

Test $\$=$ TRIM\$(Test\$)

IF LEN(Test $\$$ ) $=0$ AND AC $\$=$ "VALID" THEN GOTO Enterfilename

IF LEN(Test $\$$ ) $=0$ THEN Abortline

IF LEN(Test\$) > Namelength THEN

BEEP

DISP "ERROR in NAME ENTRY - max " "Namelength;" chars, you have "; DISP LEN(Test\$): "

WAIT 1.8

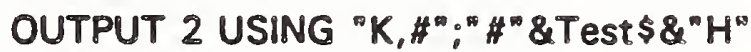

GOTO Efn

END IF

IF POS(Test\$," ") $)>1$ THEN

Test $\$=$ Test\$ $\left[1\right.$, POS(Test\$, $\left.\left."{ }^{\circ}\right)-1\right]$

Maskflag $=1$

ELSE

Maskflag $=0$

END IF

FOR $\|=1$ TO LEN(Test\$)

Ascii_num $=$ NUM(Test $\$[1])$

SELECTT Ascii_num

CASE 65 TO $90,95,97$ TO 122,48 TO 57

IAllowed characters

CASE ELSE

BEEP

DISP "ERROR in NAME ENTRY -ILLEGAL CHARACTERS, TRY AGAIN."

WAIT 9.8

OUTPUT 2 USING ${ }^{"} K_{0} \#^{\infty}{ }^{\infty}{ }^{\infty} \#^{\infty}$ \&Test $\$ \&^{D} H^{\infty}$

GOTO Efn 
END SELECT 


\begin{tabular}{|c|c|c|}
\hline \multirow[t]{3}{*}{$\begin{array}{l}\text { BL-114A } \\
(5-90)\end{array}$} & \multirow{3}{*}{$\begin{array}{l}\text { U.S. DEPARTMENT OF COMMERCE } \\
\text { NATIONAL INSTITUTE OF STANDARDS AND TECHNOLOGY } \\
\text { BIBLIOGRAPHIC DATA SHEET }\end{array}$} & $\begin{array}{l}\text { 1. PUBUCATION OA REPORT NUMBEA } \\
\text { NIST } 3978\end{array}$ \\
\hline & & $\begin{array}{l}\text { 2. PEAFOAMING ORGANIZATION REPOAT NUMBEF } \\
\text { B91-0277 }\end{array}$ \\
\hline & & $\begin{array}{l}\text { 3. PUBUCATION DATE } \\
\text { DECEMBER } 1991\end{array}$ \\
\hline
\end{tabular}

4. TITEAND SUBTTIE

AWAMS Users Manual

5. AUTHOR(S)

S.M. Chesnut, N.G. Paulter

6. PERFOAMINO ORQANIZATION (IF JOINT OR OTHER THAN MIST, SEE INSTRUCTIONS)

U.S. DEPARTMENT OF COMMEAC:

MATIONAL INSTITUTE OF STANDARDS AMD TECHMOLOOY

BOULER, COLORADO $800303-3329$

7. CONTRACT/GRANT NUMBER

8. TYPE OF AEPORT AND PERIOD COVERED

9. SPONSORING ORCANIZATION MAME AND COMPLIE ADDRESS (STREET, CTT, STATE, ZIP)

Army TMDE Support Group, Bldg. 5435

Redstone Arsenal, Alabama 35898

10. SUPPLEMENTARY NOTES

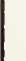
1. ABSTAACT (A 2OQ-WOAD OA LESS FACTUAL SUMMARY OF MOST SIONIFICANT INFORMATION. IF DOCUMENT INCLUDES A SIGNIFICANT BIBLOGAAPHY OA
WTERATUAE SUAVEY, MENTION IT HEAE)

The theory and operation of an upgraded version of the NIST Automatic Waveform Analysis and Measurement System is described. This system, the AWAMS, was commissioned by the Army Primary Standards Laboratory to facilitate measurement comparability with NIST. The AWAMS has been installed at the Redstone Arsenal, Alabama.

12. KEY WOPAS (6 TO 12 ENTRIES; ALPHABETICAL OADER; CAPITALZE ONLY PAOPER MAMES; AND SEPARATE KEY WORDS BY SEMICOLONS) acquisition; calibration; deconvolution; jitter; pulse parameters; waveform

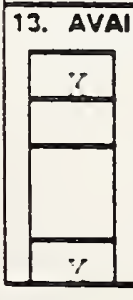

ABIUTY

UNUMITED

FOR OFFICIAL DISTRIBUTION. DO NOT RELEASE TO NATIONAL TECHMICAL INFORMATION SERVICE (NTIS).

ORDER FROM SUPERINTENDENT OF DOCUMENTS, U.S. OOVERMMENT PRINTINO OFFICE, WASHINGTON, DC 20402

ORDER FROM NATIONAL TECHMICAL IMFORMATION SERVICE (NTIS), SPRINGFIELD, VA 22161.

14. NUMBER OF PRINTED PAGES

$$
323
$$

15. PRICE

$$
\text { A14 }
$$


1 0 



